\title{
The cranial anatomy of Tenontosaurus tilletti Ostrom, 1970 (Dinosauria, Ornithopoda)
}

\section{Andrew Thomas}

\begin{abstract}
Tenontosaurus tilletti Ostrom, 1970, historically assigned to 'basal Iguanodontia,' is a species of bipedal herbivore from the Lower Cretaceous (Aptian-Albian). Previous publications on the anatomy of the species have consisted of a cursory account of specimens collected in the Cloverly Formation of the Bighorn Basin of Montana, as well as a more detailed description of the postcranial skeleton by Forster (1990). To date, the skull of $T$. tilletti remains poorly described due to lack of research and poorly preserved specimens.

The present study is an attempt to rectify the situation with material referable to Tenontosaurus tilletti, collected from southeastern Oklahoma. In particular, an especially well-preserved skull (OMNH 58340) of $T$. tilletti was CT-scanned and virtually separated into its component elements. These elements, as well as reconstructions of the internal spaces for soft tissues, such as the endocast and cranial nerve foramina, are herein described and illustrated in detail. This description is used to conduct a novel systematic analysis. The analysis strongly supports the genus Tenontosaurus, as well as its position relative to 'hypsilophodonts' and iguanodontians, and largely agrees with previous analyses.
\end{abstract}

D. Andrew Thomas. Department of Biology, Sam Noble Oklahoma Museum of Natural History, University of Oklahoma, 2401 Chautauqua Avenue, Norman, Oklahoma 73072 USA. david.a.thomas-1@ou.edu

Keywords: cranial anatomy; description; computed tomography (CT); ornithopod; Iguanodontia; Tenontosaurus

Submission: 9 December 2013. Acceptance: 17 April 2015

\section{INTRODUCTION}

Tenontosaurus tilletti (Ostrom, 1970) lived in the middle, temporally and phylogenetically, of the ornithopods, a group of highly successful dinosaurs. The clade Ornithopoda began in the Jurassic with a group of small, facultatively bipedal herbivores, historically named 'Hypsilophodonti- dae.' This small grade of ornithopod continued throughout the Cretaceous (Norman et al., 2004), and gave rise to the massive hadrosaurs of the Late Cretaceous (Horner et al., 2004), as well as the intermediate, non-hadrosaurian iguanodontians, of which $T$. tilletti has been recovered as a basal member (Norman, 2004). Throughout most of their tenure, the Ornithopoda ranked as the most

Thomas, D. Andrew. 2015. The cranial anatomy of Tenontosaurus tilletti Ostrom, 1970 (Dinosauria, Ornithopoda). Palaeontologia Electronica 18.2.37A: 1-99

palaeo-electronica.org/content/2015/1178-skull-of-tenontosaurus

Copyright: June 2015 Society of Vertebrate Paleontology. This is an open access article distributed under the terms of the Creative Commons Attribution License (creativecommons.org/licenses/by/4.0/), which permits unrestricted use, distribution, and reproduction in any medium, provided the original author and source are credited. 
diverse herbivorous taxon (Weishampel, 1985) and, at times, the most successful group of large vertebrates, on the planet (e.g., Sereno, 1997; Winkler et al., 1997; Horner et al., 2004).

Unfortunately, the phylogeny of the ornithopods basal to Hadrosauridae is poorly resolved. Several taxa, including Tenontosaurus tilletti, have been described only partially (Ostrom, 1970; Forster, 1990). Recent analyses (Winkler et al., 1998; Butler et al., 2008) have also called into question the monophyly (previously assumed, e.g., Galton, 1974a) of the 'Hypsilophodontidae.' Furthermore, Heterodontosaurus tucki, which was considered an important outgroup for systematic analyses of ornithopods since its description by Crompton and Charig (1962), has been placed near the base of Ornithischia in recent analyses (Butler et al., 2008; Norman et al., 2011), making clearer resolution of Ornithopoda even more difficult to obtain. Therefore, resolution of ornithopod phylogeny through increased fieldwork and description of new specimens is one of the most important tasks facing researchers in Ornithischia. With regard to increased resolution, the postcranial anatomy of ornithopods is fairly generalized and differs largely only in size and presumed locomotor adaptations (Norman et al., 2004; Norman, 2004; Horner et al., 2004). Their skulls, however, contain key features that have been linked to their efficacy as herbivores and, therefore, their biological success: a pleurokinetic hinge system (Norman, 1984) and increasingly specialized dentition (Weishampel, 1983, 1984; Norman and Weishampel, 1985; Norman, 2004).

Not only do the skulls of ornithopods display a wide array of morphological variation (e.g., Norman et al., 2004; Norman, 2004; Horner et al., 2004), a substantial aid to systematic analysis, but they also provide a wealth of information on the interrelationships of the various components of the skull, e.g., cranial myology, neurology, and masticatory processes (e.g., Ostrom, 1961; Witmer and Ridgely, 2009; Norman et al., 2011). Unfortunately, due to its tenuous connection to the rest of the body, the skull is rarely preserved articulated or even associated with the skeleton, sometimes famously so (McIntosh and Berman, 1975). In addition, the skull is most often preserved severely distorted because of its hollow nature (e.g., Galton, 1973; Wang and $\mathrm{Xu}, 2001)$.

An important example of this trend is provided by Tenontosaurus tilletti, whose skull is currently known almost entirely from a description of a single specimen (YPM 5456) from the Cloverly Formation
(Aptian-Albian) of Montana (Ostrom, 1970). Although almost completely preserved, the skull is laterally compressed and mosaically fractured. This compression caused certain features to be exaggerated, such as the high narial crest in lateral aspect and the acute angle between the paroccipital processes in dorsal aspect (Ostrom, 1970: figures 8,9$)$. Other, diagnostic cranial features of the species were accurately described, however, such as the long, slitlike antorbital fenestra, the supplementary inferior temporal fenestra(e) below the usual inferior temporal fenestra, and the convex posterior margin of the quadrate.

Since the initial description of $T$. tilletti (Ostrom, 1970), the only other works published on the anatomy of the species are a thesis and subsequent paper by Forster $(1985 ; 1990)$ that detailed the postcranial anatomy of specimens from the Cloverly Formation within the Bighorn Basin of Montana and Wyoming, as noted above, and a paper by Galton (1989) detailing aspects of the braincase and endocast of the species. The purpose of the present study is to provide a detailed description of a skull (OMNH 58340) that is preserved in much better condition than that described by Ostrom; three additional and hitherto undescribed skulls (OMNH 16562, OMNH 34191, OMNH 2531) are also included in the present work.

\section{OMNH 58340}

The specimen, OMNH 58340, was discovered in southeast Oklahoma (Antlers Formation, Aptian-Albian). The first part of the individual to be discovered was the middle portion of the tail, which had partially eroded from the slope of the hillside within a drained pond. During laboratory preparation, the entire skeleton, lacking many of the eroded caudal vertebrae, was found to be preserved beautifully, with very little distortion, and included rarely preserved elements such as the hyobranchials, terminal manual phalanges, preatlantes, and atlantal ribs.

The skull, in particular, is worthy of note. The endocranium and left side of the cranium are threedimensionally preserved and pristine, with few exceptions (notably, some disintegration in the maxilla). The right side of the rostrum (maxilla, premaxilla, lacrimal, nasal, prefrontal, and palatine) is preserved, disarticulated from the rest of the skull. The right suspensorium (quadrate, quadratojugal, jugal, and pterygoid) is shifted anteromedially, probably due to the same taphonomic stress that disarticulated the rostrum. The stapes (unknown 
for the species) and palpebrals are the only elements of the skull not preserved.

The complete description of the cranial anatomy of this specimen is made possible through the use of High Resolution X-ray Computed Tomography (X-ray CT). X-ray CT dramatically increases the yield of information from paleontological specimens, elucidating details that were previously available only in taphonomically or mechanically disarticulated specimens. The software used to process X-ray CT also gives researchers the ability to measure volumes of individual aspects of internal cranial anatomy, to virtually disarticulate elements, and to view various components of the specimen in ways that could otherwise be harmful. Previous X-ray CT studies have largely concentrated on the skulls of theropods (e.g., Franzosa and Rowe, 2005, Sanders and Smith, 2005, Witmer and Ridgely, 2009) and sauropods (e.g., Sereno et al., 2007), with a relative paucity in Ornithopoda.

\section{A note on the figures}

The software used to capture many of the images used and that form the basis for others has a feature that allows for the effects of perspective to be eliminated. This makes actual measurements possible in a single image that would otherwise be impossible due to the shrinking and expanding effect of perspective. The only images to which this was not applied, i.e., those in which perspective must be taken into account, are the photographs. The line drawings, computer-generated images, and CT images are free of distortion due to perspective.

\section{METHODS}

The skull and jaws of OMNH 58340 were taken to the High-Resolution X-ray Computed Tomography Facility at the University of Texas at Austin for CT scanning (www.digimorph.org). The scan was performed using a machine built by BioImaging Research, Inc., Lincolnshire, Illinois (BIR, subsequently acquired by and operating as a subsidiary of Varian Medical Systems, Inc., Palo Alto, California). The high-energy subsystem of the scanner was used, employing a 450-kV tungsten X-ray source, a turntable, and a 512-channel cadmium-tungstate solid-state linear array detector. The software used to reconstruct the data collected is custom to the facility; however, the various routines to correct the data are programmed using the IDL programming language. These routines correct

\section{Abbreviations Used}

$$
\begin{array}{ll}
\text { A - Angular } & \text { Pal - Palatine } \\
\text { AoF - Antorbital Fenestra } & \text { Psp - Parasphenoid } \\
\text { Art - Articular } & \text { P - Parietal } \\
\text { Bo - Basioccipital } & \text { PoP - Paroccipital Process } \\
\text { Bsp - Basisphenoid } & \text { Po - Postorbital } \\
\text { Co - Coronoid } & \text { Pa - Prearticular } \\
\text { D - Dentary } & \text { Pd - Predentary } \\
\text { Ect - Ectopterygoid } & \text { Pf - Prefrontal } \\
\text { Eo - Exoccipital } & \text { Pmx - Premaxilla } \\
\text { F - Frontal } & \text { Pro - Prootic } \\
\text { ITF - Inferior Temporal } & \text { Pt - Pterygoid } \\
\text { Fenestra } & \\
\text { J - Jugal } & \text { Q - Quadrate } \\
\text { L - Lacrimal } & \text { Qj - Quadratojugal } \\
\text { Lsp - Laterosphenoid } & \text { Spl - Splenial } \\
\text { Mx - Maxilla } & \text { Sq - Squamosal } \\
\text { Na - Naris } & \text { STF - Superior Temporal } \\
\text { N - Nasal } & \text { Fenestra } \\
\text { Op - Opisthotic } & \text { So - Supraoccipital } \\
\text { Or - Orbit } & \text { Sa - Surangular } \\
\text { Osp - Orbitosphenoid } & \text { Vo - Vomer } \\
\end{array}
$$

\section{Cranial Nerves:}

$$
\begin{array}{ll}
\text { I - Olfactory Nerve } & \text { VI - Abducens Nerve } \\
\text { II - Optic Nerve } & \text { VII }- \text { Facial Nerve } \\
\text { III - Oculomotor Nerve } & \text { VIII - Vestibulocochlear Nerve } \\
\text { IV - Trochlear Nerve } & \text { IX - Glossopharyngeal Nerve } \\
\text { V - Trigeminal Nerve } & \text { X - Vagus Nerve } \\
\text { V }- \text { Ophthalmic Branch } & \text { XI - Spinal Accessory Nerve } \\
\mathrm{V}_{2}-\text { Maxillary Branch } & \text { XII - Hypoglossal Nerve } \\
\mathrm{V}_{3}-\text { Mandibular Branch } &
\end{array}
$$

for various artifacts including rings, beam hardening, X-ray drift, and rotation errors.

Scanning was completed at a diameter of 250 $\mathrm{mm}$ for both the skull and jaws. Images of 346 slices of the mandibles and other disarticulated cranial elements (hyoids, right maxilla, right palatine, right prefrontal, and predentary) and 425 slices of the skull were taken, both at $1 \mathrm{~mm}$ slice thickness. A second, higher resolution scan of the area of the braincase was subsequently performed, with a diameter of $110 \mathrm{~mm}$. Three hundred forty-eight slices were made at $0.5 \mathrm{~mm}$ slice thickness from the anteriormost tip of the orbitosphenoid to the posteriormost tip of the basioccipital. 
Sectioning of the skull to isolate the components described below was completed with VGStudio Max software, v2.1 (Volume Graphics $\mathrm{GmbH}$, Heidelberg, Germany), which was used to trace the outline of each articulated cranial element, slice by slice. An option within VGStudio Max was also applied that allows the user to "force isotropic resampling." With this option selected, the software interpolates an additional three slices between each pair of slices (an exact average of the two original images, and two additional images averaged between the new middle image and the original images on either side), which creates smoother curves when rendering. The same software was then used to assemble these slices into threedimensional, virtual models from which measurements could be taken. The isolation process created artifacts in the virtual elements generated, usually consisting of an easily recognizable, irregular undulation in the contact surface between elements, which was created by small inconsistencies in the placement of the outline of the element from slice to slice.

Finally, to provide context for the following description of OMNH 58340, comparisons are made throughout with the original description by Ostrom (1970), as well as with other ornithopods evenly distributed in recently recovered phylogenetic analyses (e.g., Norman et al., 2004; Norman, 2004; Horner et al., 2004; McDonald, 2012), both basal and derived with respect to Tenontosaurus tilletti. A revised phylogeny based on a novel analysis of cranial characteristics of ornithopods, along with previously published postcranial characters (Norman, 2004), follows after the description. Taxa in this analysis (Appendix 1) were selected on the basis of phylogenetic relevance to $T$. tilletti and for the availability of reasonably detailed cranial descriptions.

Some arbitrary linguistic conventions are needed to refer to multiple taxa that, collectively, do not represent monophyletic groups. By reference to the phylogenetic analyses presented by Norman et al. (2004), Norman (2004), and Horner et al. (2004), I have adopted the following usage, with terms accompanied by specific examples in the text (a list of the taxa included in each category can be found in Appendix 1). The historical 'Hypsilophodontidae' is probably paraphyletic (e.g., Winkler et al., 1998; Brown et al., 2013), and the taxa traditionally recovered in the clade are herein referred to as 'basal ornithopods.' 'Basal iguanodontians' is used in the text to refer to members of the clade Iguanodontia, excluding hadrosaurs. Of these, branching points for Tenontosaurus, Zalmoxes robustus, Dryosaurus altus, Cumnoria prestwichii, and Theiophytalia kerri are placed low on the tree (Norman, 2004), and these taxa are generally referred to as 'more basal' iguanodontians. The remaining taxa (Appendix 1) branch higher on the tree (Norman, 2004), and are collectively referred to as 'higher,' or 'more derived' iguanodontians. Finally, the remaining three taxa are referred to, collectively, as hadrosaurs. Exceptions to these groupings are noted in the text.

Abbreviations used: $\mathrm{AMNH}-\mathrm{American}$ Museum of Natural History, New York; BB-Buffalo Bill Museum, Cody, Wyoming; BMNH-Natural History Museum (British Museum of Natural History), London; MCZ-Museum of Comparative Zoology, Harvard University, Cambridge, Massachusetts; OMNH-Sam Noble Oklahoma Museum of Natural History, University of Oklahoma, Norman, Oklahoma; YPM-Peabody Museum of Natural History, Yale University, New Haven, Connecticut; YPMPU-Peabody Museum of Natural History, Yale University, New Haven, Connecticut (originally in the collections of Princeton University).

\section{GEOLOGICAL SETTING}

Specimens attributed to species of Tenontosaurus have been recovered from the Trinity Group (Aptian-Albian) of north central Texas and the Antlers Formation (Aptian-Albian) of Texas and Oklahoma. Three units represent the Trinity Group in north-central Texas (in ascending order): the Twin Mountains, the Glen Rose, and the Paluxy formations. The Twin Mountains and Paluxy are lithologically similar, terrigenous units. The intervening Glen Rose limestone is a shallow, transgressive marine unit that pinches out to the north and east, in the absence of which the undifferentiated Twin Mountains and Paluxy are collectively termed the Antlers Formation (Winkler et al., 1990). Although the base of the Twin Mountains has not been constrained directly, it is assumed to be no older than Aptian, based on the presence of Aptian marine invertebrates in its seaward lateral equivalents (Jacobs and Winkler, 1998). The base of the Albian was identified near the base of the Glen Rose, also indicated by the presence of particular marine invertebrates (Jacobs and Winkler, 1998), and the mid-Albian Walnut Formation overlies the Paluxy. Therefore, the Antlers Formation, again the lateral equivalent of the Twin Mountains and Paluxy, can only be refined to Aptian-Albian (for additional stratigraphic discussion, see e.g., Winkler et al., 1990; Jacobs and Winkler, 1998). 
The specimen of Tenontosaurus tilletti described herein, OMNH 58340, was collected in the late fall and winter of 2000-2001, from the Antlers Formation, OMNH Locality V821, approximately $1 \mathrm{~km}$ northeast of the better documented V706 (Cifelli et al., 1997; Davis et al., 2008; Davis and Cifelli, 2011), in Atoka County in southeastern Oklahoma. Locality V821 is also the locality from which Sauroposeidon proteles Wedel et al., 2000 was collected, but this specimen of $T$. tilletti was collected from stratigraphically higher sediment (approximately 3-5 m higher).

The Antlers Formation in the area of V821 consists of silty and sandy claystones, as well as fine- to coarse-grained sandstones. Sandstones are moderately to poorly sorted and unindurated to clayey to locally carbon-cemented. Strata are irregularly cross-bedded and ferruginous with some conglomerates (Manley, 1965). These sediments are interpreted as being derived from highlands to the north and west (the Ouachita, Wichita, and Arbuckle ranges) and subsequently deposited in fluvial, deltaic, and strandplain environments on a broad coastal plain near the paleocoastline of the proto-Gulf of Mexico (Hobday et al., 1981). The paleoclimate is interpreted as subtropical and semiarid (Winkler et al., 1990). Based upon the estimated dip of the rock, as well as the extent of lateral outcrops and the published thickness estimates from nearby counties, Rennison (1996) estimated the section for the nearby V706 to be $150 \mathrm{~m}$ thick. The lithology of V821, in particular, tends toward ferruginous sandstones.

\section{SYSTEMATIC PALEONTOLOGY}

\author{
Order ORNITHISCHIA Seeley, 1888 \\ Suborder ORNITHOPODA Marsh, 1881 \\ Family IGUANODONTIA Dollo, 1888 (sensu \\ Sereno, 1986) \\ Tenontosaurus tilletti Ostrom, 1970
}

Holotype. AMNH 3040: a partial skeleton, lacking pectoral girdle, cervicals, and skull.

Paratypes. YPM-PU 16338: a partial skeleton, lacking pectoral girdle, cervicals, and skull; YPM 5456: a partial skeleton with skull.

Referred Specimens. MCZ 4087, 4166, 4205, 4385, 4388, 4390, 7556-7558; OMNH 2526, 2531, 4164, 8137, 10132, 10144, 16562, 16563, 32838, $32842,32845,32847,34191,34782,58340$, 62990, 63525; BB 1;YPM-PU 16514; YPM 4882, 4904, 5099, 5117, 5146, 5195, 5299, 5399, 5410, $5411,5413,5416,5417,5422,5424,5426-5428$, 5457-5471, 5473-5481, 5483, 5523, 5533-5535;
AMNH 3010-3014, 3017, 3020, 3022, 3023, 3031, 3034, 3043-3045, 3050, 3061-3063, 5854.

Horizon and Distribution. Little Sheep Mudstone and Himes Member of the Lower Cretaceous (Upper Aptian-Lower Albian) Cloverly Formation (Units V, VI, and VII of Ostrom, 1970) of the Bighorn Basin area of northern Wyoming and southcentral Montana; Lower Cretaceous Antlers Formation (Aptian-Albian) of southeast Oklahoma.

Revised Cranial Diagnosis (emended from Ostrom, 1970; for revised postcranial diagnosis, see Forster, 1990). Skull with very large external nares, long slitlike antorbital fenestra and supplementary inferior temporal fenestra beneath the usual lateral fenestra. Orbit roughly triangular, with dorsoventrally straight anterior and sharply rounded posterior margins (emended by this study), and larger than either lateral fenestra. Premaxilla, which nearly encircles the nares, flares inferiorly into broad, U-shaped edentulous beak, opposed by shallow, horseshoe-shaped predentary with pseudo-tooth projections along upper margin. Mandibles bear a long, curved retroarticular process. Quadrate long, very narrow transversely; posterior margin convex rather than concave. Paroccipital processes hook-shaped, and downturned at extremity. Dentary teeth with very prominent vertical keel, maxillary teeth without keels but with numerous nonparallel, subequal minor ridges.

The following characteristics of Tenontosaurus tilletti were recovered in the systematic analysis described below. Characters are followed by "(character number:state present)," found in Appendices 2 and 3 . The first set of character states are presumed plesiomorphies of $T$. tilletti: lack of contact between the jugal and quadrate (20:0), lack of quadrate foramen (25:0), large quadratojugal (26:1), contact between nasal and lacrimal (28:0), articulation of the jugal with the posterior maxilla (33:0), lack of quadrate notch $(37: 0)$, lack of contact between the parietal and prootic (49:1), dorsal edge of the ilium above the ischial peduncle not thickened and beveled (87:0), straight ischial shaft (92:0), and ischial shaft flattened in cross section (93:0). Tenontosaurus tilletti shares the following apomorphic characters with other members of Iguanodontia: frontal contributes to less than half of dorsal orbital rim (8:1), parallel dorsoventral borders of the dentary (54:1), single surangular foramen (69:2), dorsal neural spines rectangular with height more than twice width (72:1), manus digit III bearing three phalanges $(84: 1)$, and a pubic shaft shorter than the ischium, with no pubic symphysis (91:1). The following char- 
acters are apomorphic to the genus Tenontosaurus: nasal overlapped by nasal process of the premaxilla (13:1), postorbital process comprises posteriormost tip of jugal (22:1), triangular outline of parietal in dorsal aspect (45:1), denticulate occlusal margin of predentary (62:1), laterally reflected boss on anterior margin of the scapula $(75: 1)$, laterally compressed and bar-like prepubic process of the pubis (90:1), and an obturator process present near the midshaft of the ischium (94:1). Finally, the following suite of characters is autapomorphic to T. tilletti: lack of premaxillary teeth (3:1), length of suture between frontals and parietal equal to about twothirds the combined transverse width of the frontals (7:1), axial length of the nasals one and one-half times axial length of the frontals (10:1), posteriorly curved and distally flared scapular blade (74:2), distal phalanges of manus digit II claw-like and digit III a nub (83:2), and straight distal shaft of the femur (96:1). Two autapomorphies for $T$. tilletti (internal contacts of the squamosal with the jugal and of the basioccipital with the laterosphenoids) were not coded in this analysis as their status is unable to be investigated in the other taxa analyzed at this time.

\section{DESCRIPTION OF THE CRANIAL ELEMENTS}

The skull of OMNH 58340 comprises the complete braincase, left and right suspensoria (quadrates, quadratojugals, jugals, pterygoids, ectopterygoids), left side of the rostrum, and left and right ceratobranchials and mandibles with the predentary. The right rostrum is preserved disarticulated. The palpebrals and stapes were not preserved.

The braincase and the left side of the rostrum and suspensorium are preserved in articulation. The right portion of the suspensorium is shifted such that, in posterior aspect, the right quadrate is oriented vertically (Figure 1) and the anterior tip of the right jugal is displaced about $2 \mathrm{~cm}$ both dorsally and laterally (Figure 2). The right maxilla, together with portions of the right lacrimal, nasal, and premaxilla, was found disarticulated in close proximity to the rest of the skull, shifted dorsally in apparent conjunction with the corresponding suspensorium. The only other elements of the cranium preserved completely disarticulated are the right prefrontal and palatine and pieces of the left sclerotic ring. The mandibles were found articulated with the cranium, on either side of the ceratobranchials. The predentary, originally thought to be lost, was later discovered within the thorax.
The skull will be described in maxillofacial, neurocranial, and mandibular segments. The terms anterior and posterior, dorsal and ventral, and medial and lateral are used in the description. Comparisons between taxa are made throughout and a list of references and specimens used in comparison is compiled below (Appendix 1). Systematic characters and character states, which are listed below (Appendix 2, Appendix 3), are noted in the text as "(Character \#:\#)," with the first symbol indicating the character and the second indicating state. Interested readers can download threedimensional models of each of the elements of the skull in stl format (Appendix 4). Finally, the CT scans generated for this study are available for download (Appendix 5).

\section{Maxillofacial Series}

Ostrom (1970, p. 87) described the skull of Tenontosaurus tilletti as "long and deep, in pronounced contrast to the low profile of Camptosaurus [Theiophytalia Brill and Carpenter, 2006]." Although true, in overall form (Figure 2) the skull is somewhere between the tall, Roman-nosed profile figured in Ostrom's original description (Ostrom, 1970, figure 8) and the flat skull roof of Theiophytalia (Gilmore, 1909, figure 2). As originally restored (Ostrom, 1970, figure 9), Tenontosaurus tilletti closely resembles the transversely compressed, deep skull of Hypsilophodon foxii (Galton, 1974a, figure 7a). However, the skull of OMNH 58340 is nearly hexagonal in posterior aspect, resulting from the wide spacing of the distal ends of the quadrates (Figure 1).

Premaxilla. The left premaxilla of OMNH 58340 is preserved in its entirety. The posterolateral corner of the beak and part of the maxillary process of the right premaxilla are preserved in articulation with the disarticulated right maxilla (Figure 3). Most of the beak of this specimen is poorly preserved, making it necessary to combine the observations made of this specimen with the disarticulated right premaxilla of a slightly smaller individual, OMNH 34191, from the same locality (Figure 4).

In lateral aspect (Figure 2), the premaxilla appears U-shaped, extending back in a gracile nasal process and a sheetlike maxillary process, which form the dorsal, anterior, and ventral borders of the nares. Medially, the paired premaxillae meet with a smooth, nearly vertical abutting suture along the length of the nasal process (Figures 4.2, 5, 6). In dorsal aspect, the paired premaxillae meet to form a rounded point anteriorly (Figure 5), then flare out posteriorly, creating the edentulous beak 

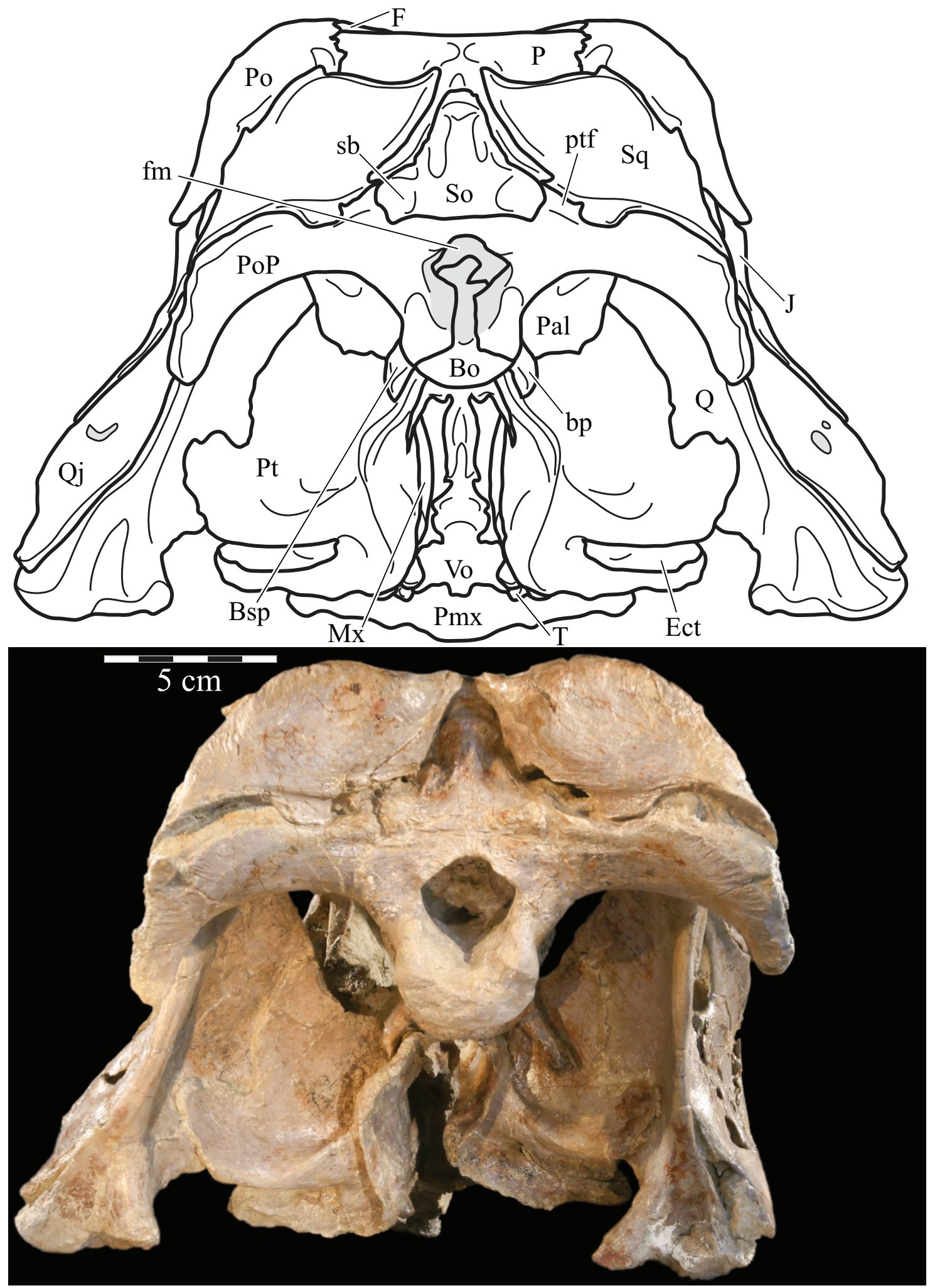

FIGURE 1. Posterior skull schematic (above) and photograph (below) of OMNH 58340. The schematic was reconstructed by digitally mirroring the left side of the suspensorium in order to approximate the actual appearance of the skull. The two images are set to the same scale, demonstrating the amount of displacement in the right side. Anterior is into the page. Abbreviations: bp - basipterygoid process; fm - foramen magnum; ptf - posterior temporal foramen (obscured); sb - squamosal boss. 

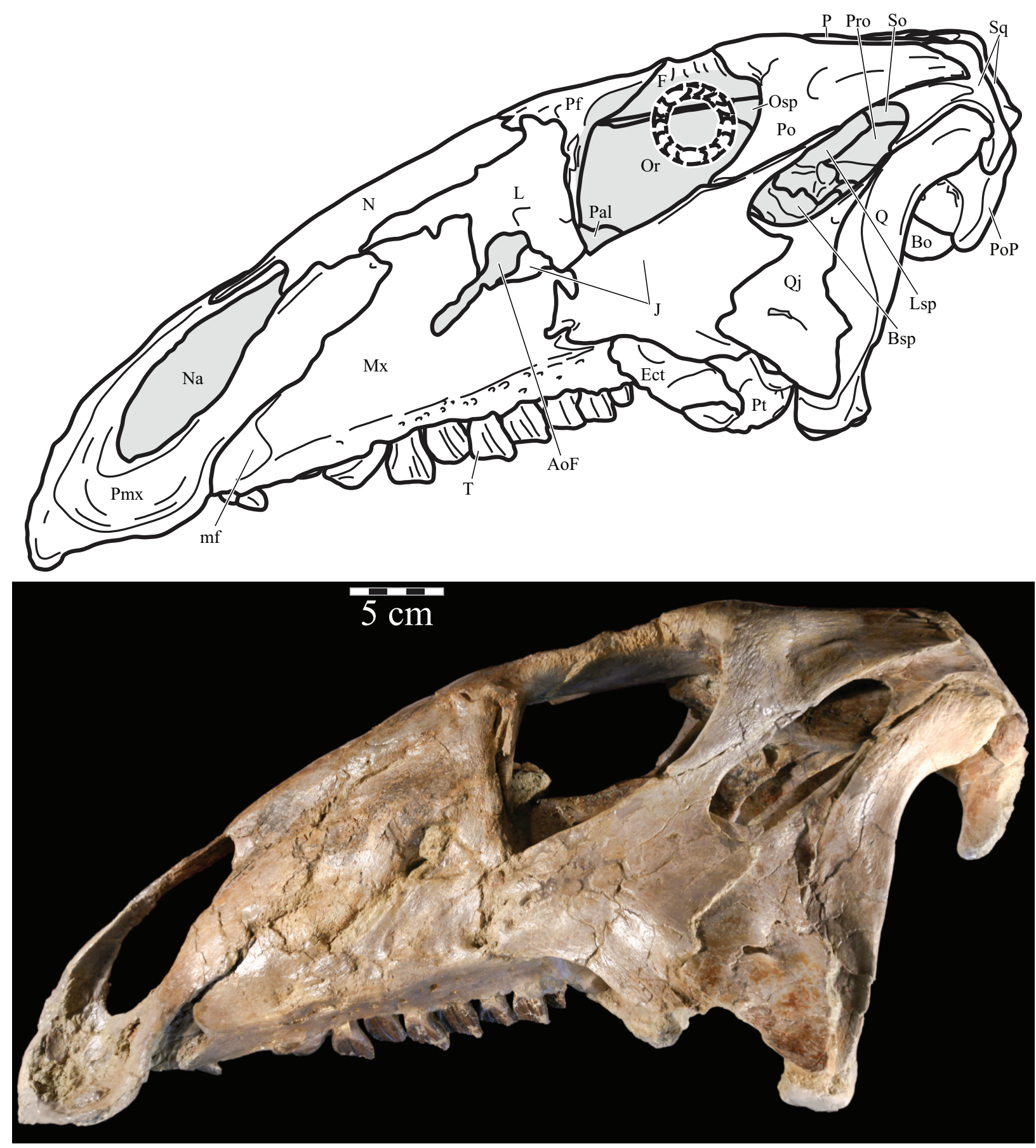

FIGURE 2. Left lateral skull schematic (above) and left skull photograph (below) of OMNH 58340. The skull is angled at the 'alert position' indicated by the horizontal semicircular canal. Natural fenestrae are shaded gray. Dashed outline denotes conjectural sclerotic ring. Anterior is to the left. Abbreviation: mf - maxillary foramen.

characteristic of basal iguanodontians. Posteriorly, the lateral edges of the beak curve medially to become the ventral edge of the maxillary process (Figure 2).

The nasal process of the premaxilla arises from a stout dorsal projection at the front of the beak and quickly thins along its lateral and ventral edges (Figures 2, 4.1). After the initial constriction, the process retains a roughly square cross-section as it arches back, then tapers dorsoventrally, ending just before the antorbital fenestrae (Figure 5, Character 4:0). The upper border of the naris is 


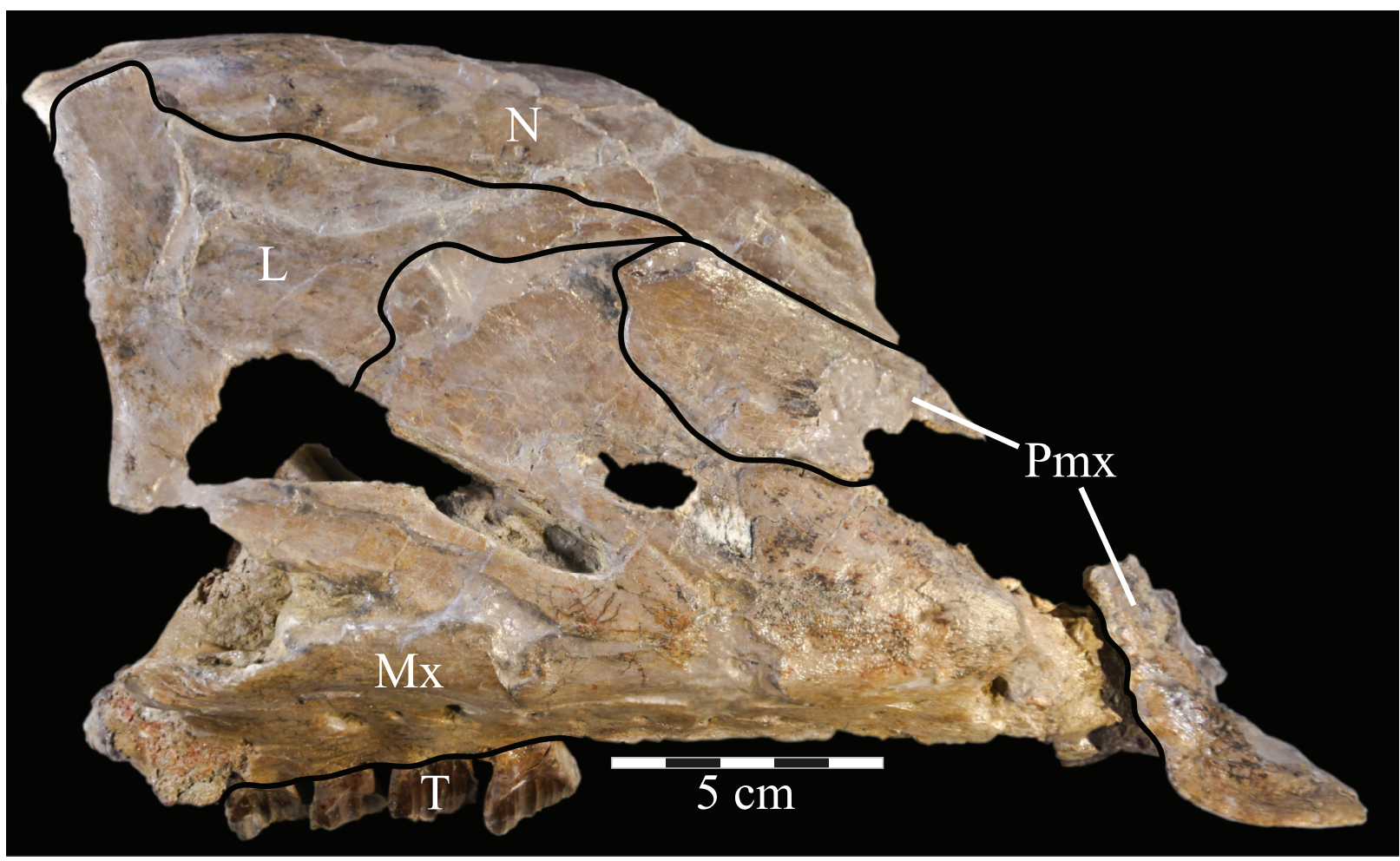

FIGURE 3. Photograph of the lateral side of the disarticulated right maxilla and other associated elements of OMNH 58340. The exclusion of the maxilla from contact with the nasal is apparent, although there is some separation, probably taphonomic, of the premaxilla and lacrimal. Dotted line indicates uncertain sutural placement between the nasal and maxilla.

formed in front by the nasal process and by the nasal behind (Figures 2, 5), where the paired nasal processes extend between the paired nasals. The nasal processes first contact the nasals laterally then fit into dorsomedially open grooves (Character 13:1). This also appears to be the case in Hypsilophodon foxii (Galton, 1974a), but in more derived taxa, such as Iguanodon bernissartensis and Mantellisaurus atherfieldensis, the nasals overlap the premaxillae dorsally (Norman, 1980; 1986).

There is a short neck in the maxillary process as it extends back from the posterior edge of the beak, before the process flares in height (Figure 2). This neck forms the dorsal border of the maxillary foramen, which opens outward in the maxilla below. The laminar maxillary process then extends back the same distance as the nasal process, overlapping the dorsal edge of the maxilla (Figures $2,5)$. The rounded posterior tip of the maxillary process makes contact with the lacrimal, excluding the maxilla from contact with the nasal (Figure 2, Character 1:1). Contact between maxilla and nasal is present in Tenontosaurus dossi (Winkler et al., 1997) and more basal taxa but is not present in all taxa more derived than T. tilletti (e.g., Iguanodon bernissartensis, Figure 7) except Zalmoxes robustus (Weishampel et al., 2003).

The beak of the premaxilla laterally covers the anterior process of the maxilla, which dorsally overlays the plate-like head of the vomer (Figure 8). In Hypsilophodon foxii, the anterior process of the maxilla and the head of the vomer insert into a groove on the medial surface of the premaxilla (Galton, 1974a, figure 4B), while in Tenontosaurus dossi (Winkler et al., 1997) and Eolambia caroljonesa (Head, 2001) only the anterior process of the maxilla was noted to do so. However, since the vomer was not included in the description of those taxa, it is possible that an arrangement similar to that found in $T$. tilletti is actually present.

The beak of the premaxilla forms the anterior and anterolateral portions of the hard palate while the vomer makes up the center (Figure 6). In more derived iguanodontian taxa, such as Iguanodon bernissartensis and Mantellisaurus atherfieldensis, the premaxilla appears to form the entire hard palate and the state of its articulation with the vomer is unknown (Norman, 1980; 1986).

The occlusal margin of the premaxilla is level with the occlusal margin of the maxillary teeth (Fig- 


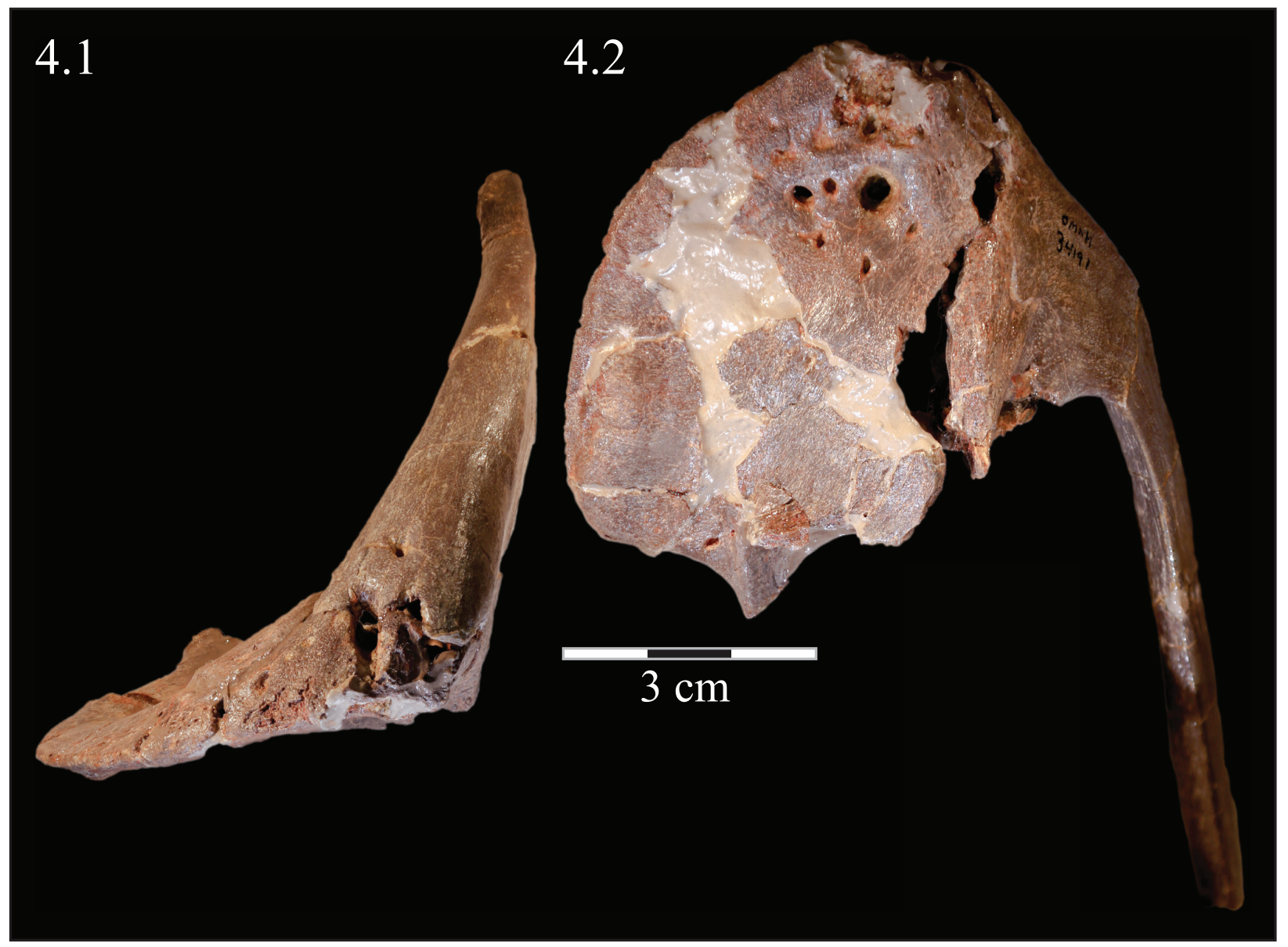

FIGURE 4. Ventral (4.1) and anterior (4.2) photographs of the right premaxilla of OMNH 34191. In 4.1, anterior is out of the page and lateral is left. In 4.2 , lateral is to the left and anterior is up.

ure 2, Character 5:0), as is the case in iguanodontians basal to Iguanodon bernissartensis (Figure 7). In contrast to its sister taxon, Tenontosaurus dossi, and other more basal forms (e.g., Hypsilophodon foxii, Thescelosaurus neglectus), the premaxillae of Tenontosaurus tilletti bore no teeth (Character 3:1; Winkler et al., 1997; Galton, 1974a, 1974b). Due to poor preservation, it is impossible to tell definitively on either specimen (OMNH 58340 and $\mathrm{OMNH} 34191$ ) whether or not there were denticles or other ornamentation along the ventral or anterior occlusal margins of the beak, which would have helped support or define a keratinous beak and corresponded to similar structures present on the predentary. However, there is a short groove that runs between two possible denticles on the anterior edge of OMNH 58340. On OMNH 34191 (Figure 4), there are several different-sized foramina on the anterior and anteroventral surfaces, and the lateral and posterior portions of the occlusal edge are smooth and rather sharp.
A boss is weakly present on the ventral surface of the beak of OMNH 58340 and more distinctly on OMNH 34191, which corresponds with the dorsal flattening of the dentary processes of the predentary below (see description of the predentary below).

Vomer. The median vomer is the longest element in the skull, stretching from the middle of the beak in front to the back of the orbit behind (Figure 9). It appears, especially posteriorly, to be a paired element, fused at the midline. Although it is well preserved in OMNH 58340, comparison of the vomer of Tenontosaurus tilletti with those of other ornithopods is made difficult by its poor preservation in other specimens and, therefore, scant description.

Anteriorly, the bone is dorsoventrally flat and transversely expanded into a ventrally concave plate that makes up the central third of the lower surface of the beak (Figure 6). The anterior process of the maxilla is interposed between the plate of the vomer below and the beak of the premaxilla 

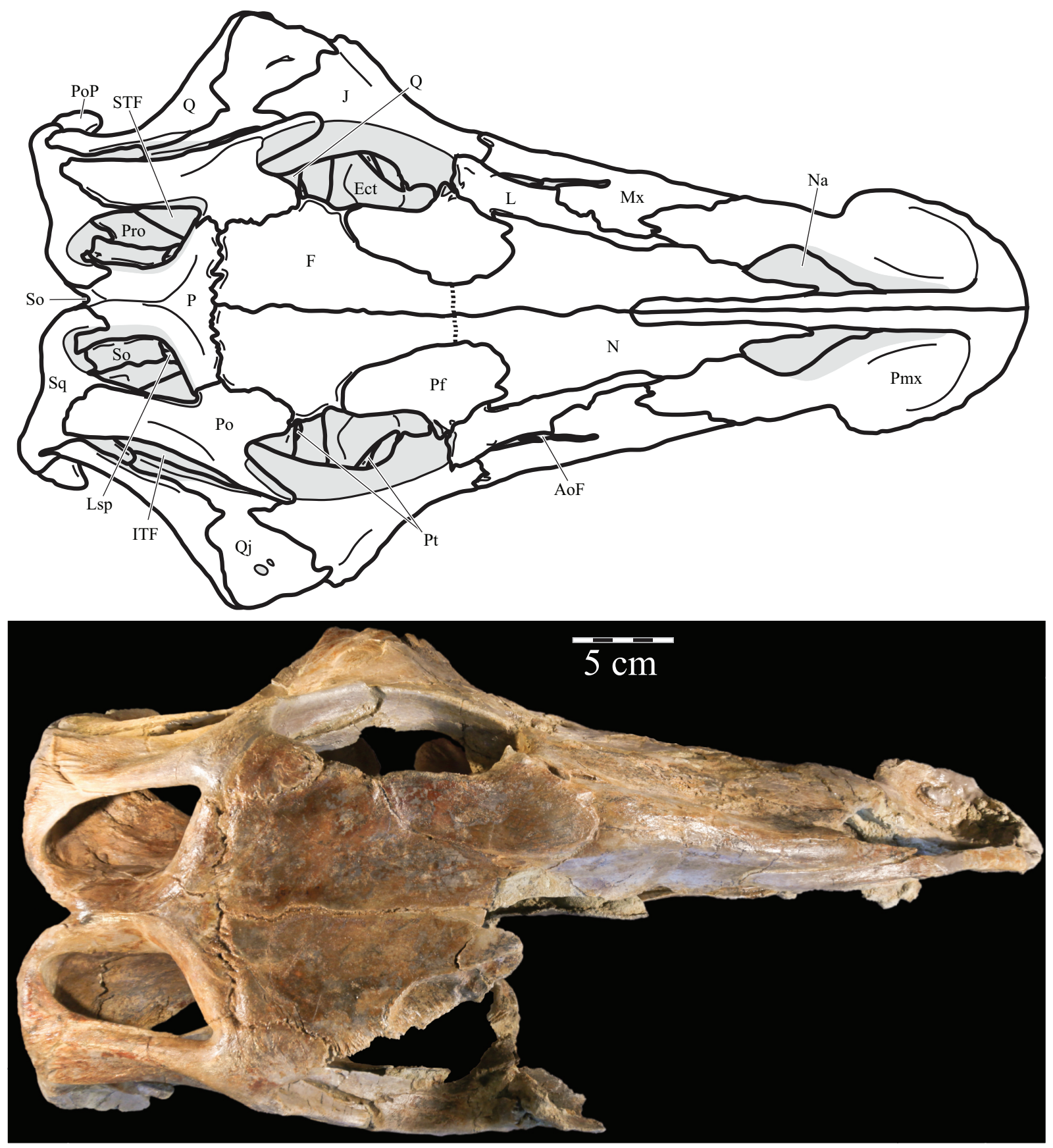

FIGURE 5. Dorsal skull schematic (above) and photograph (below) of OMNH 58340. The schematic was reconstructed by digitally mirroring the left side of the rostrum and suspensorium in order to approximate the actual appearance of the skull. Dotted line at the anterior edge of the frontals indicates uncertain sutural placement. Natural fenestrae are shaded gray. Anterior is to the right.

above (Figure 8). Along its length, the vomer does not articulate again with the dental ridge of the maxilla, but stays in close association with it (Figure 6), in apparent contrast with all other ornithopods in which this element is recognized or its traces preserved along the medial surface of the maxilla (e.g., Hypsilophodon foxii, Iguanodon ber- nissartensis, Edmontosaurus regalis), although this could be due to poor preservation in those specimens.

From the plate in front, the concave ventral surface of the vomer extends about halfway down the length of the element (Figure 6). The concavity ends in a pointed fossa, which is angled back and 

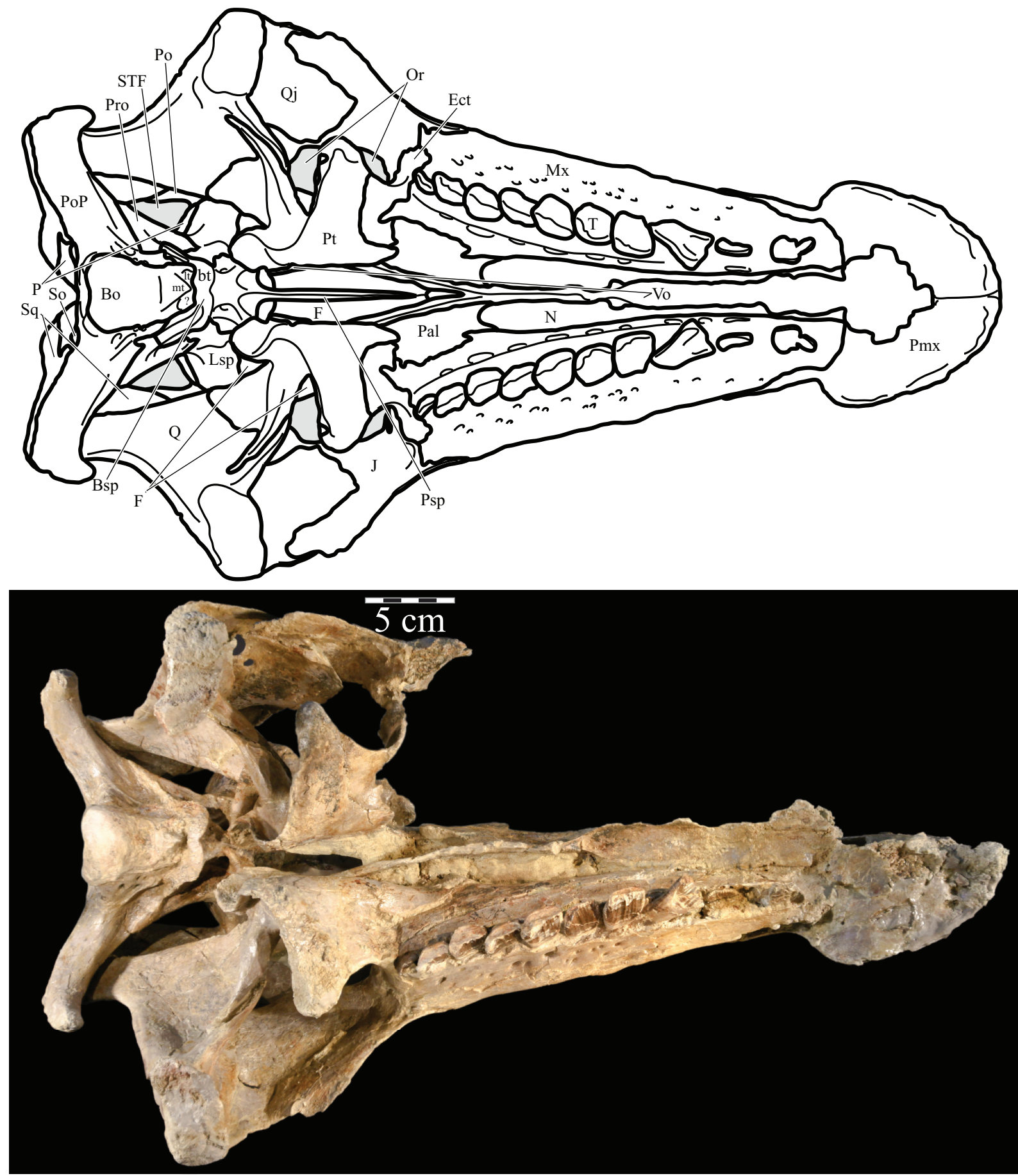

FIGURE 6. Ventral skull schematic (above) and photograph (below) of OMNH 58340. The schematic was reconstructed by digitally mirroring the left side of the rostrum and suspensorium in order to approximate the actual appearance of the skull. Natural fenestrae are shaded gray. Anterior is to the right. Abbreviations: bt - basal tubera of the basisphenoid; It - lateral tubercle of the basioccipital; mt - median tubercle of the basioccipital; ? - possible second lateral tubercle of the basioccipital.

marks the beginning of the median ventral ridge that continues posteriorly until it is split into the paired posterior processes that form the remainder of the element (Figures 6, 10.1, Appendix 4:
Vomer). The ventral surface of this median ridge is rough and rounded, suggesting either the presence of soft tissue attachment or of continued ossi- 


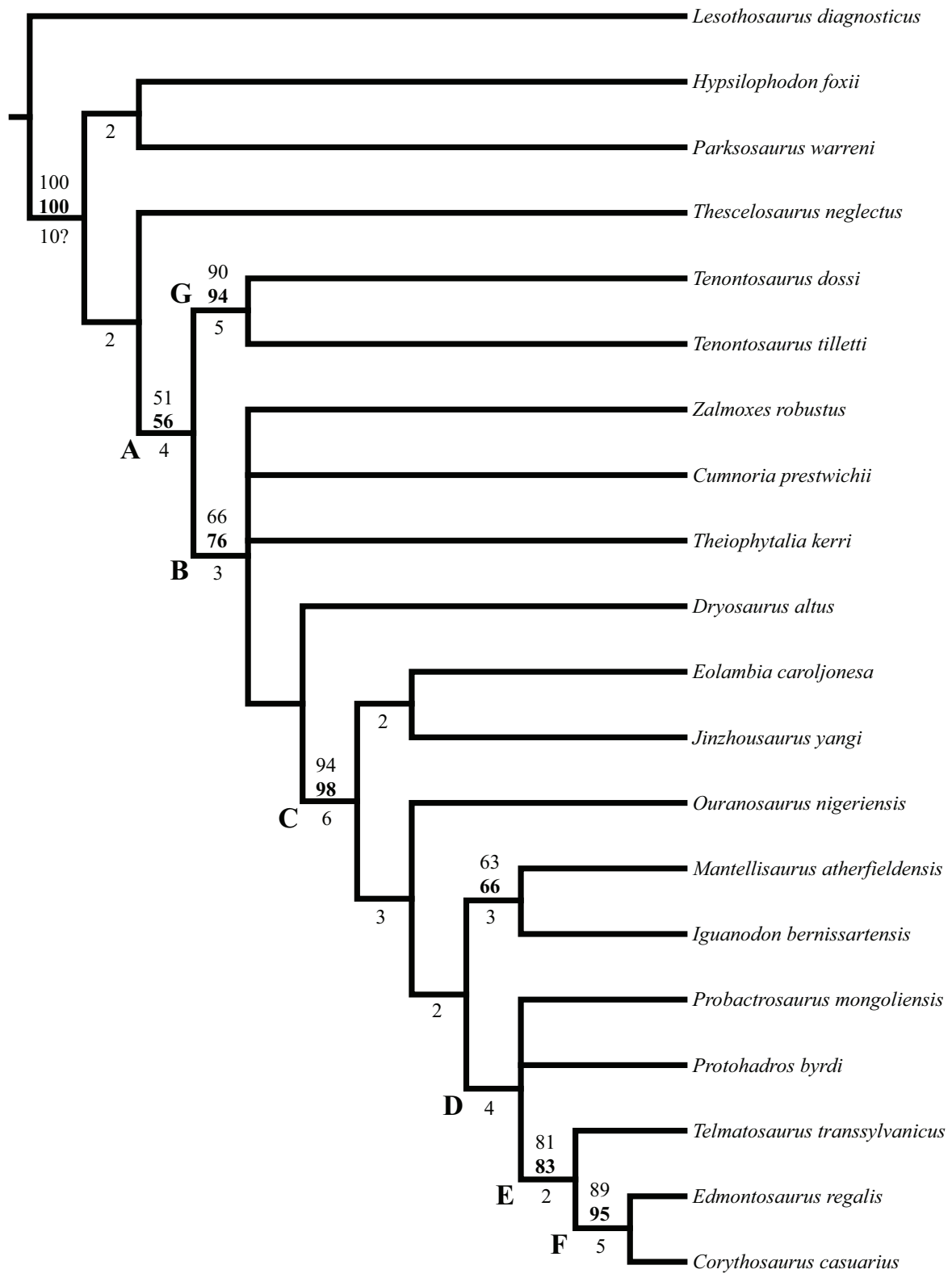

FIGURE 7. Strict component consensus tree of selected members of Ornithopoda included in this analysis. Lesothosaurus diagnosticus is the outgroup. Bremer support values are located below, while Bootstrap and Group Present/ Contradicted (GC) support values are located above the branches immediately preceding the node to which they refer. GC values are in bold. Certain nodes are noted with letters referenced in the text. Optimization was performed in PAUP* version 4.0b10 (Swofford, 2002) and checked in TNT (Goloboff et al., 2008).

fication farther ventrally; it does not appear to be a clean termination (Figure 9).

Dorsally, a narrow, rounded median ridge begins at about the level of the neck of the maxillary process of the premaxilla. Here, the vomer shifts from being dorsoventrally to transversely thin (Figures 6, 11.1). Posteriorly, the median ridge gradually gives way to a median furrow, at which point a pair of sharp ridges arise from the dorsal surface of the element that become taller behind (Figure 11.1). At their tallest point, about halfway along their length (Figure 9), the ridges spread apart and diverge into the posterior processes. Here, the laminae of the palatines (see description of the palatine below) lean in over the paired posterior processes of the vomer, which are gently convex outward, resembling parentheses in coronal cross-section (Figure 12). 


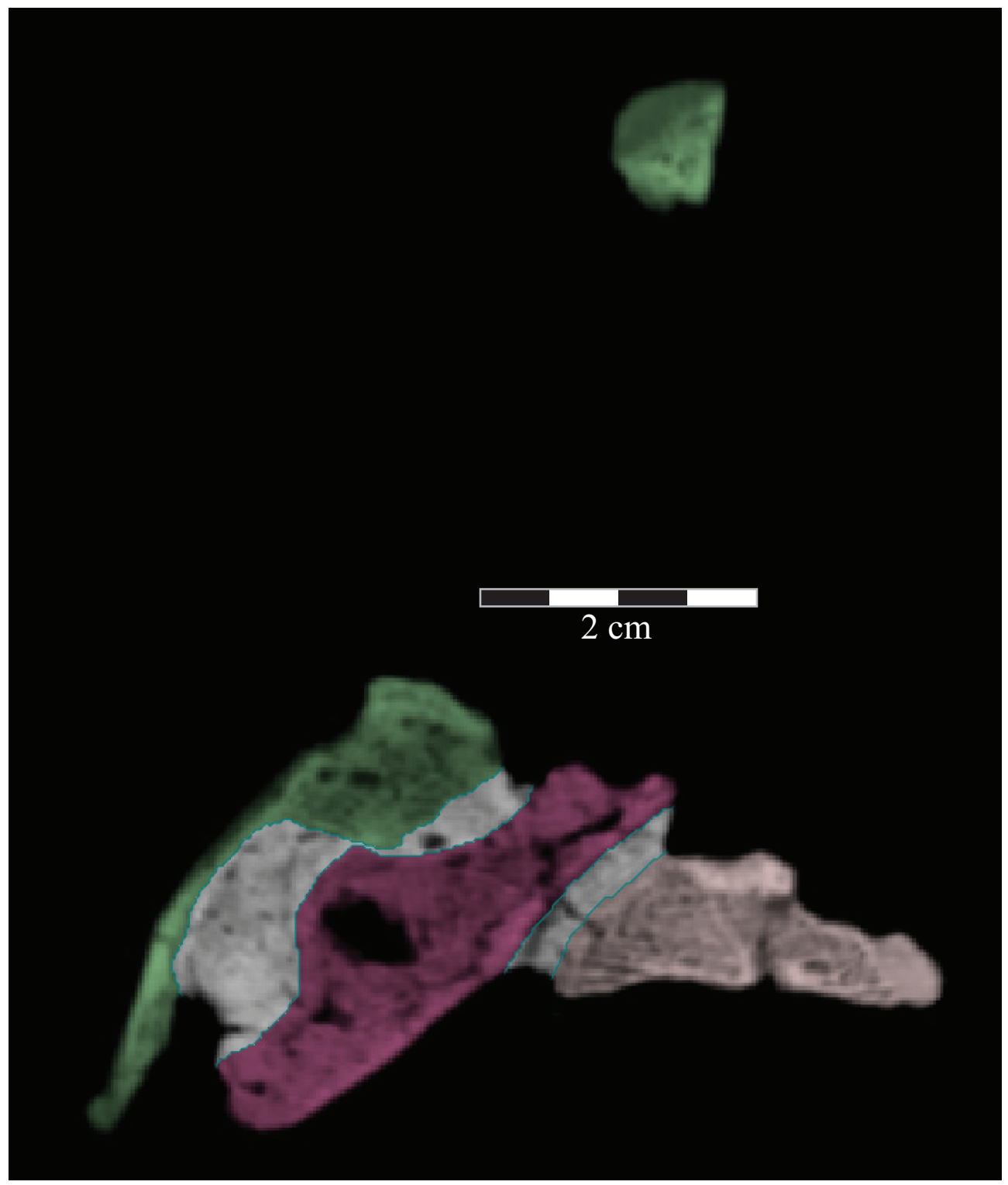

FIGURE 8. Coronal CT image, taken at the level of the open naris, of the left premaxilla (green, left), maxilla (pink, middle), and the vomer (cream, bottom) of OMNH 58340 showing the nature of their articulation. The maxilla likely fitted in closer articulation with the premaxilla. Faint green lines are an artifact of the program used to define the element on each image. Anterior is into the page.

Each posterior process quickly shortens behind its tallest point (Figure 9). The processes then rotate outward, so that the dorsal edges become ventral, and the ventral edges dorsal. The posterior end of each process flares again after a dorsoventral constriction, appearing triangular in medial aspect, with the dorsal edge forming the hypotenuse and the ventral edges making up the legs (Figure 9). Medially, the posterior triangle forms a small ridge that extends down and out into the probable passage of the pharynx (Figure 9, Appendix 4: Vomer). The posterior triangular area of the vomer also medially overlaps the body and palatine and medial processes of the pterygoid (Figures 9, 10, see below). Articulation of the vomer with the pterygoid was described as probable for many ornithopods (e.g., Iguanodon bernissartensis), but noted in only Dryosaurus altus and Edmontosaurus regalis.

Palatine. The palatine is a paired element with two main components, a lateral process and a sagittally oriented lamina (Figures 13, 14). In OMNH 58340, the left palatine is preserved in articulation while the right is preserved completely disarticulated. 

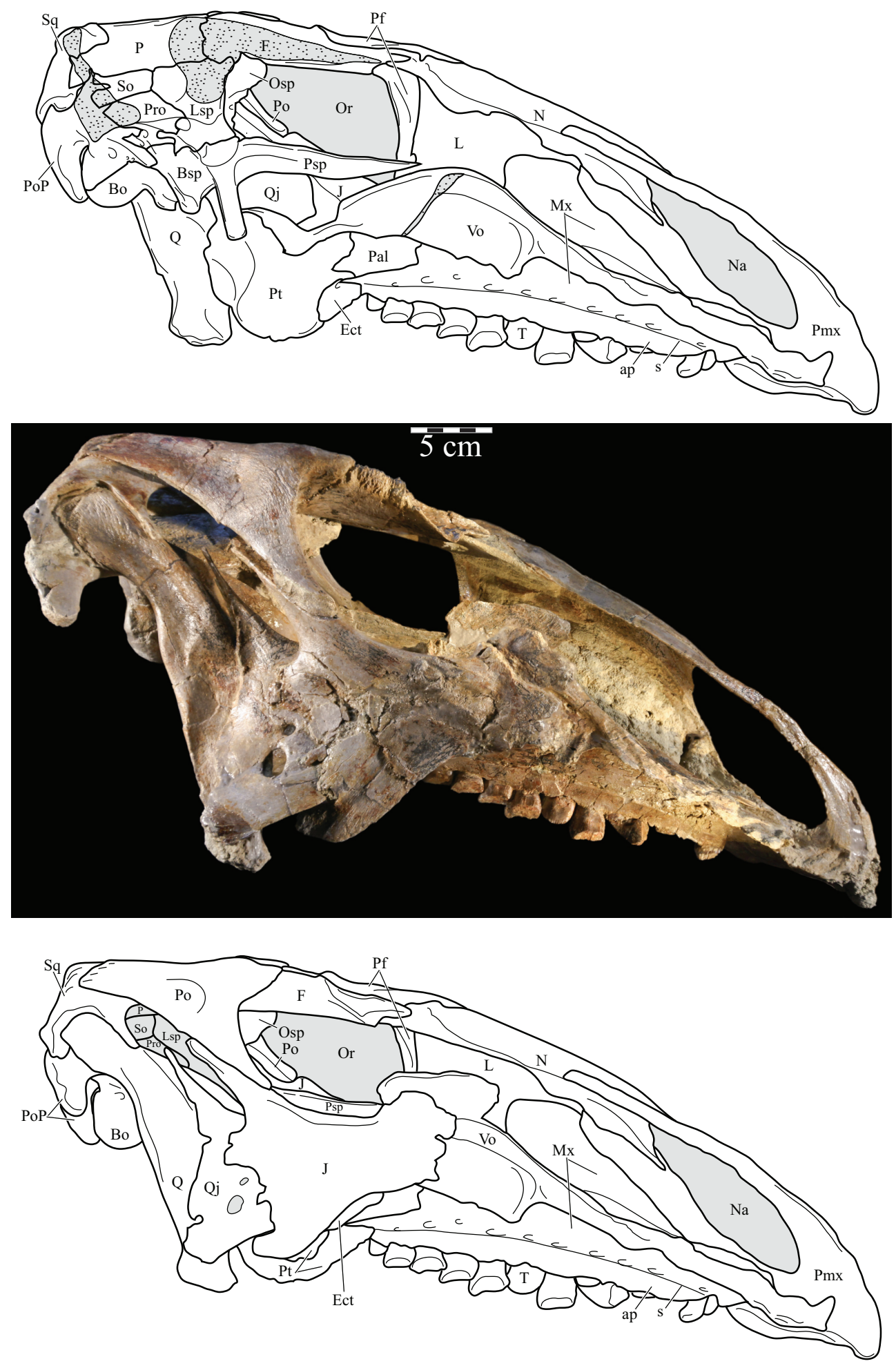

FIGURE 9. Medial skull schematic (above), right skull photograph (middle), and right skull schematic (below) of OMNH 58340. Parts of the vomer, frontal, parietal, laterosphenoid, squamosal, supraoccipital, exoccipital/opisthotic, and prootic, as well as the entirety of the right quadrate, quadratojugal, and jugal, have been removed from the medial skull schematic in order to show the structure of the braincase and its orientation with the rest of the skull. The broken texture in gray in the upper schematic indicates the sectioning of elements necessary to view the endocranium. The skull is angled at the 'alert position' indicated by the horizontal semicircular canal. Natural fenestrae are shaded gray. Anterior is to the right. Abbreviations: ap - alveolar parapet; s - sulcus. 


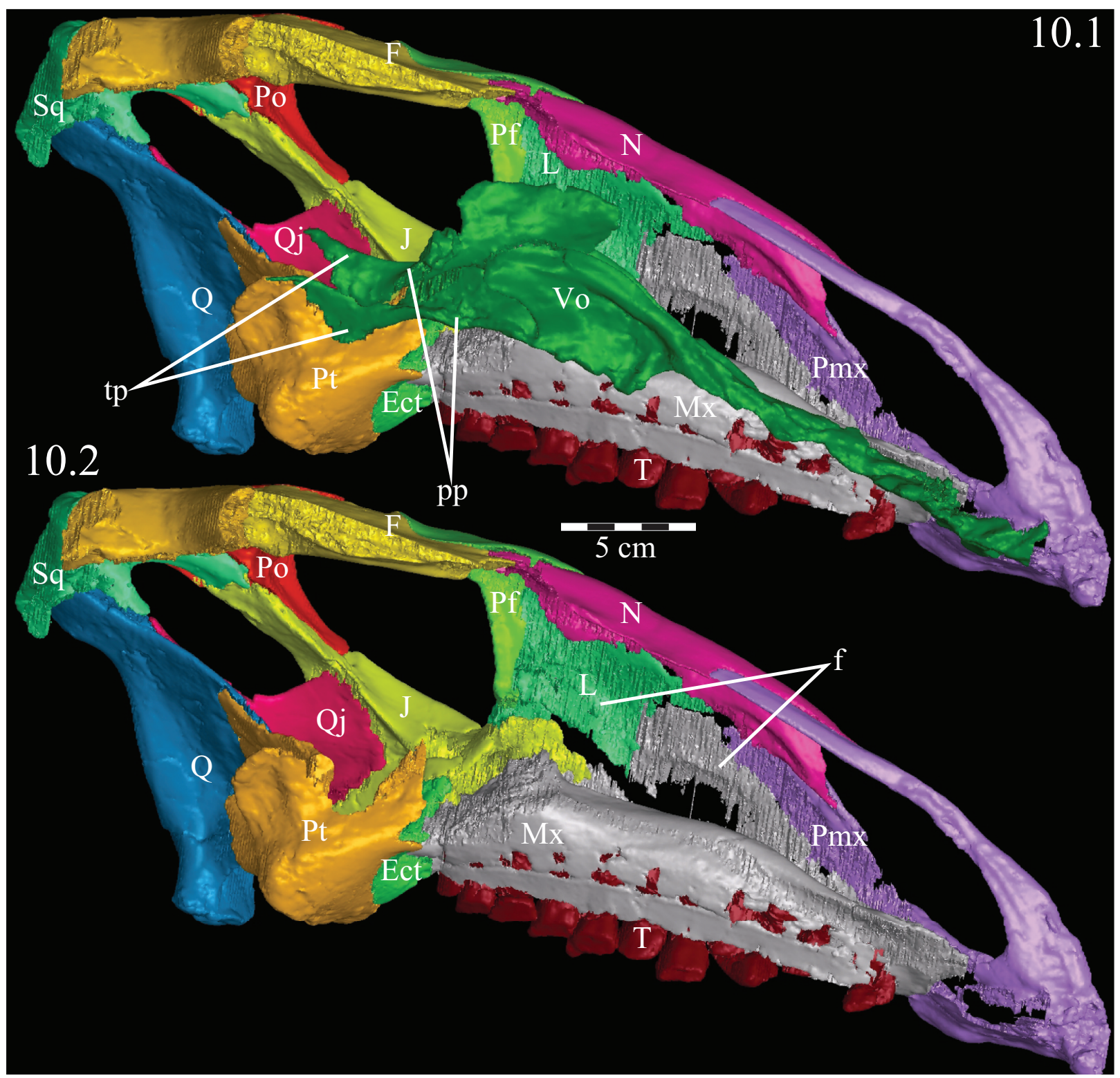

FIGURE 10. Medial view of the left side of the virtual skull of OMNH 58340 with the vomer present (10.1), allowing a view of the articulation of the vomer with the pterygoid, and with the palatine and vomer removed (10.2), allowing a view of the joints between the maxilla, lacrimal, prefrontal, jugal, ectopterygoid, and pterygoid. The vertically striated texture present on the visible surfaces of many elements, notably the lacrimal, maxilla, and premaxilla, is an artifact of the process used to isolate CT images of each element from the remainder of the data set. Abbreviations: $f$ - flange; $\mathrm{pp}$ - posterior processes; tp - triangular processes.

The medial lamina of the palatine is bootshaped in medial aspect, with an anterior process forming the toe (Figure 13.1, Character 29:0). This shape appears to be intermediate between basal ornithopods and more derived iguanodontians (e.g., Hypsilophodon foxii, Iguanodon bernissartensis, respectively). Transversely, the lamina is very thin, although it thickens in the anterior process and along its posteroventral edge due to contributions from the lateral process (Figure 13.2). The dorsal edge of the lamina is weakly sinusoidal (Appendix 4: Palatine). This edge expands into a digitate articular surface that connects to the palatine process of the pterygoid and the ectopterygoid posteriorly and the dental ridge of the maxilla ventrally (Figures 14, 15, Character 18:0). A single small foramen begins anterodorsally on the medial side of the lamina and opens posteroventrally in a concavity at the juncture of the posterior and ventral ridges of the lateral process. Medially, the lam- 


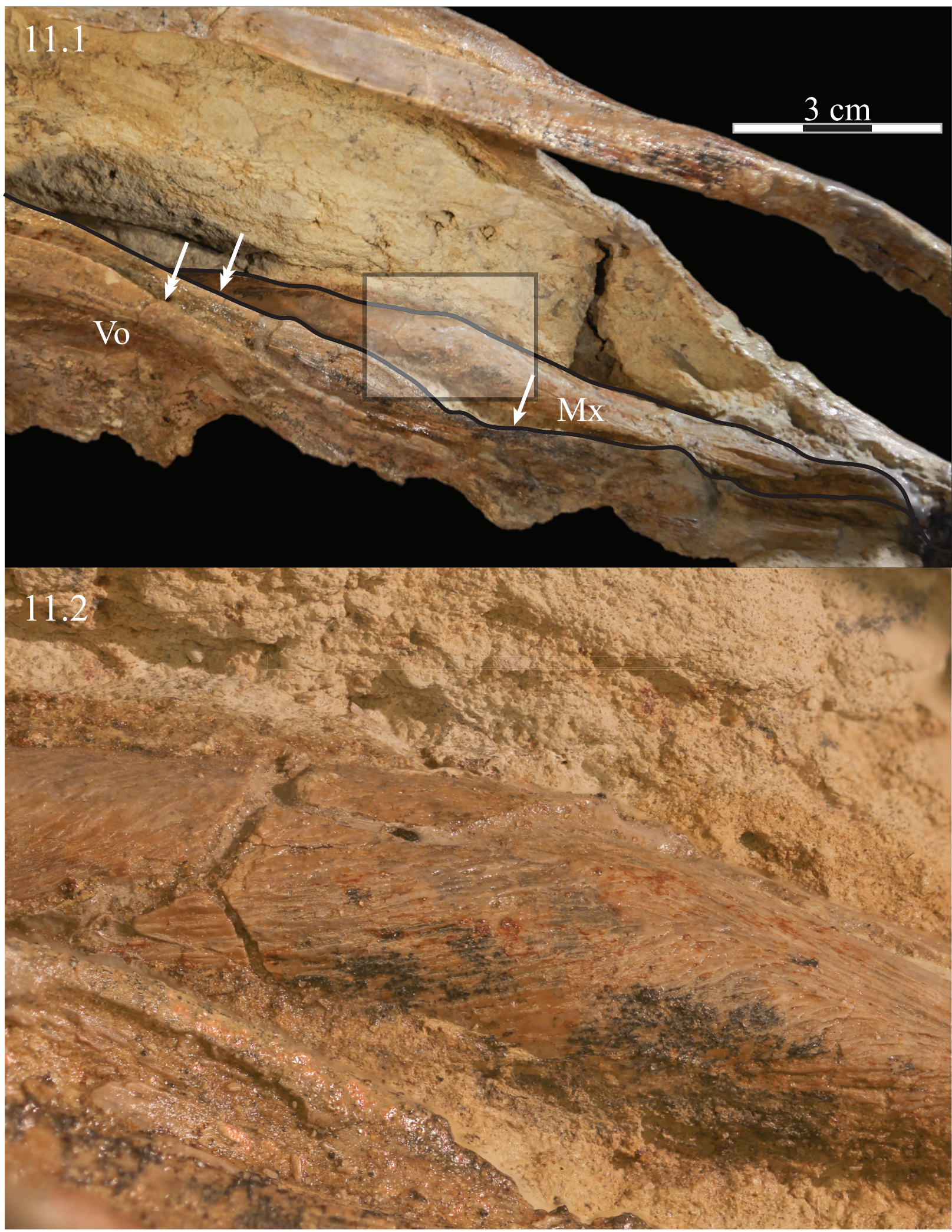

FIGURE 11. Photographs of the medial aspect of the vomer (foreground) and dental ridge of the maxilla (background, just below matrix) of OMNH 58340 (11.1) and showing the fine grain on the dorsal surface of the maxilla (11.2). Single headed arrow indicates the median ridge of the vomer. Double headed arrows indicate the paired sharp ridges of the same element. The area figured in 11.2 is indicated by the shaded area in 11.1. Anterior is to the right. 


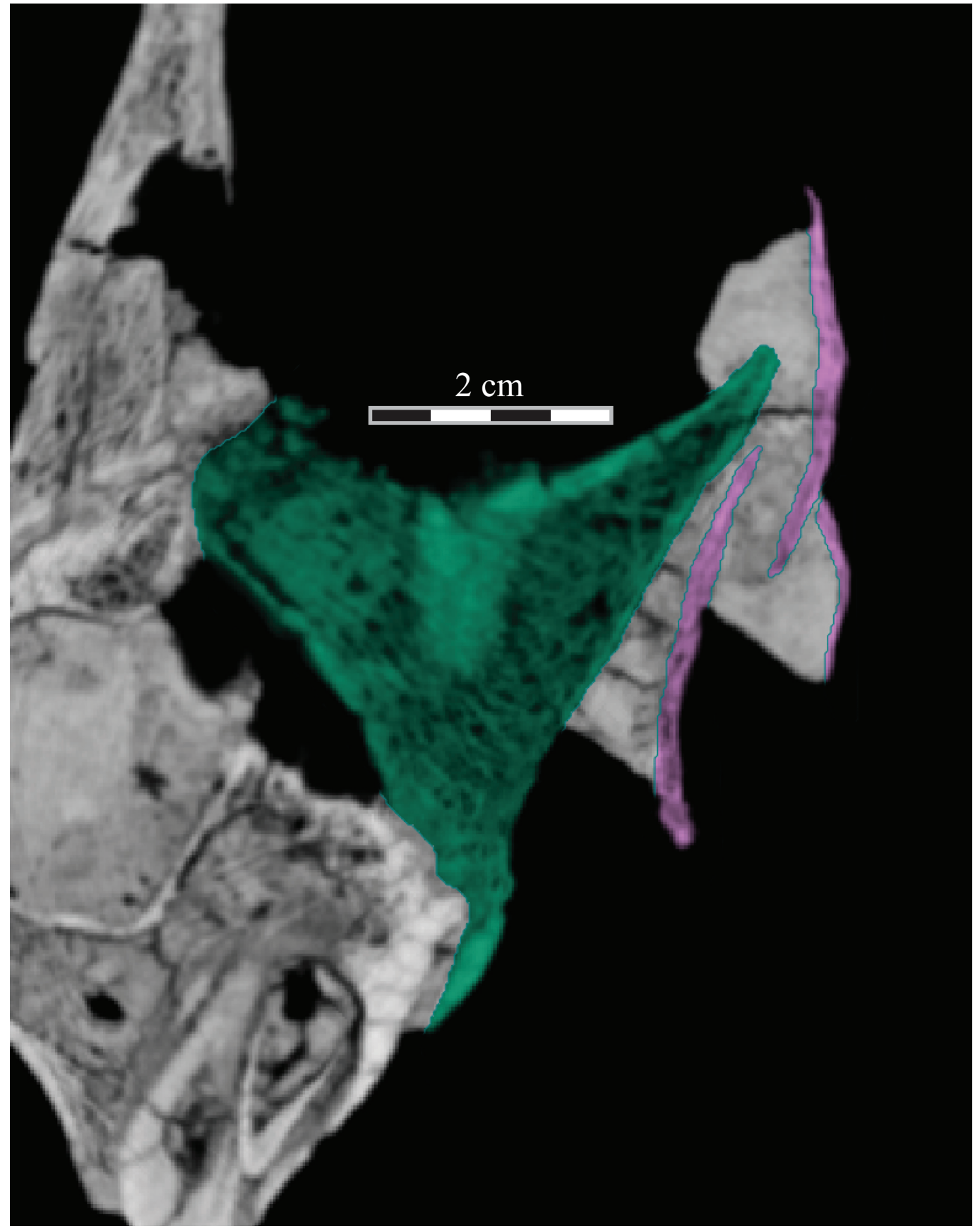

FIGURE 12. Coronal CT image, taken at the level of the posterior termination of the antorbital fenestra, of the paired posterior processes of the vomer of OMNH 58340. This image shows the disarticulated nature of the two halves of the posterior part of the element, as well as their shape just anterior to the opening of the orbit, between the paired palatines. Faint green lines are an artifact of the program used to define the element on each image. Anterior is into the page.

ina leans over the posterior processes of the vomer, mirroring the lateral convexity of that element (Figure 12; see above).

The lateral process extends outward from the lamina, with dorsal, anterior, and posterior ridges, which quickly smooth into an elliptical cross-section (Figures 13, 14, 15, Appendix 4: Palatine). The process continues laterally across the back of the dental ridge of the maxilla, spanning the maxillary canal, until it meets the superior anterior process of the jugal and the pedicles of both the prefrontal and lacrimal in a digitate suture (Figures 14, 15). The lamina of the palatine is almost totally obscured from view in the orbit in lateral aspect 


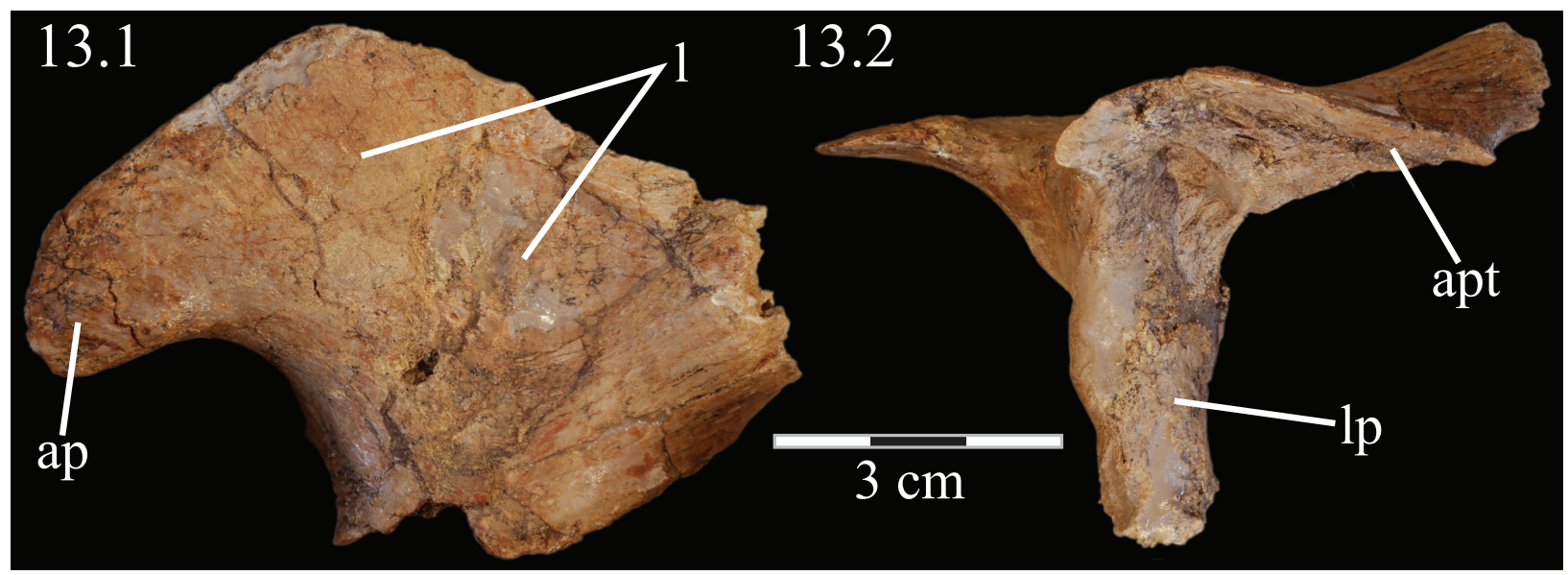

FIGURE 13. Medial (13.1) and ventral (13.2) photographs of the disarticulated right palatine of OMNH 58340. Anterior is to the left. Abbreviations: ap - anterior process; apt - articulation with pterygoid; I - lamina; Ip - lateral process.

(Figure 2), indicating that the palatine is farther forward than in other ornithopods (e.g., Hypsilophodon foxii, Iguanodon bernissartensis).

Ectopterygoid. The ectopterygoid is a simple, strap-like bone, which folds laterally almost to a right angle about its middle, thus appearing rectangular in dorsolateral aspect and C-shaped in dorsomedial aspect (Figure 14). The anterior, vertically-oriented leg of the element is thinner than the transversely oriented posterior leg. The outer anterior tip of the element appears to split into a dorsal and ventral point at its articulation with the jugal, which may create an actual foramen or which may be an artifact of preservation (Character 19:0). This lateral articulation with the jugal is a characteristic of most basal iguanodontians (e.g., Cumnoria prestwichii, Tenontosaurus dossi), which is lost in hadrosaurs (e.g. Corythosaurus casuarius, Edmontosaurus regalis). The suture between the two elements does not appear to be as strong as described by Galton (1974a) for Hypsilophodon foxii; however, the configuration of the ectopterygoid, jugal, and maxilla does not appear capable of allowing significant flexure.

There is a transverse groove running along the anterior surface of the ectopterygoid, toward the foramen mentioned above, that is filled by the posteromedial process of the maxilla (Figures 10, 14). The articulation with the maxilla occurs along the posterior edge of the latter element, in contrast to iguanodontians more derived than Iguanodon bernissartensis (e.g., Ouranosaurus nigeriensis, Figure 7) and hadrosaurs in which the ectopterygoid shelf lies much farther forward (e.g., Edmontosaurus regalis). The anterior leg likely formed the anteroventral border of the adductor chamber.
As the ectopterygoid curves back, the dorsal edge of the bone flares up in a tab with sharp anterior and posterior corners (Appendix 4: Ectopterygoid). The anterior corner overlaps the interdigitate lower posterior edge of the lamina of the palatine where it articulates with the palatine process of the pterygoid (Figure 14, Character 18:1). The only other iguanodontians in which the ectopterygoid articulates with the palatine are Tenontosaurus dossi and, possibly, Dryosaurus altus (Winkler et al., 1997; Galton, 1983). The posterior corner lies deep within the lateral fossa of the pterygoid. Behind this, the ectopterygoid moves out of the fossa and eventually overlaps the pterygoid, as the roof of the groove on the pterygoid recedes (see description of the pterygoid below).

On the lateral side of this curving area, a tubercle begins ventrally and extends back to the posterior tip of the bone, which apparently forms a functional unit with the ectopterygoid process of the pterygoid below (Figure 16). Galton (1974a, figure $5 \mathrm{C}$ ) figured a similar, but more exaggerated feature for Hypsilophodon foxii. This tubercle appears poorly ossified and may be for the connection of soft tissue or for the origin of M. pterygoideus dorsalis (Ostrom, 1961). Behind the curve, the ectopterygoid lies in the lateral fossa of the pterygoid (Figure 16).

Pterygoid. The pterygoid is a complex bone, with four main processes: quadratic, palatine, medial, and ectopterygoid (Figure 16). The medial and ventral surfaces of the element, which form a right angle with each other, are smooth. The medial surface was likely the lateral wall for the pharynx, and here the two bones in articulation resemble paired parentheses (Figure 1). Ventrally, the pterygoid 


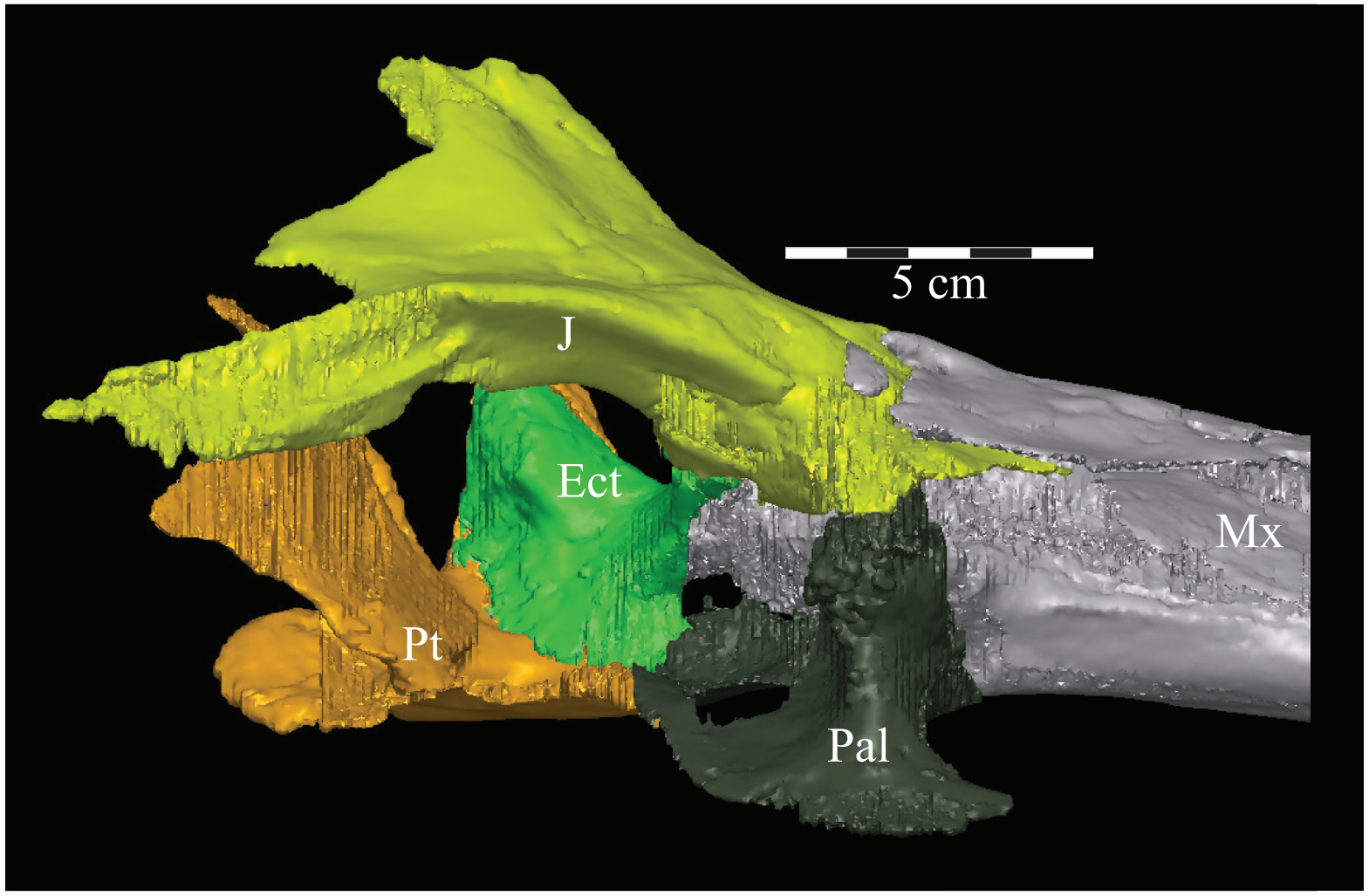

FIGURE 14. Dorsal view of the elements of the virtual palate of OMNH 58340, including the palatine, maxilla, pterygoid, and ectopterygoid, as well as the jugal, detailing the means of articulation between the various elements. Anterior is to the right.

forms the rear portion of the hard palate (Figure 6). Both pterygoids are preserved in articulation in $\mathrm{OMNH} 58340$, although the right element is shifted with the other components of the suspensorium (Figure 1).

The palatine process of the pterygoid extends forward, splitting into a larger dorsal and a smaller ventral tab (Figures 9, 10, 16, 17). Together, these articulate with the lower posterior edge of the lamina of the palatine. The ventral tab is overlapped laterally by the ectopterygoid (Figure 16). The ventral edge of the pterygoid swings laterally to become the anterior border of the ectopterygoid process, the upper surface of which is marked by a deep fossa to accommodate the back leg of the ectopterygoid. This fossa continues out to the tip of the process, which matches the rounded and rough shape of the lateral tubercle of the ectopterygoid above (Figure 16; see description of ectopterygoid above). Here, these elements form the pterygoid flange.

There is a transverse ridge on the posterior side of the pterygoid, which rises medially, before arcing upward to become the posterior edge of the medial process (Figures 1, 15, Appendix 4: Ptery- goid). The lateral concavity created by the upswing of this ventral ridge is likely the origin of the $M$. pterygoideus ventralis (Ostrom, 1961).

The medial process of the pterygoid is sigmoid in posterior aspect, with a medially convex bottom and concave top (Figure 1, Appendix 4: Pterygoid). There is no medial process in Hypsilophodon foxii, and the pterygoids are spaced farther apart in that species. For Dryosaurus altus, however, Galton (1983) described both form and spacing of the elements to be similar to that found here in Tenontosaurus tilletti.

There is a notch in the upper edge of the pterygoid between the medial and palatine processes, which is filled by the triangular tip of the posterior process of the vomer (Figures 10, 16). If an articulation with the vomer is surmised at all in ornithopods, it is suggested to occur farther forward, somewhere along the palatine process; however, poor preservation may account for this discrepancy (e.g., Norman, 1980; Galton, 1983). In OMNH 58340 , this joint appears to have been capable of some rotation about the sagittal axis.

The sheetlike quadratic process is weakly concave posteriorly (Figure 16) and extends back 


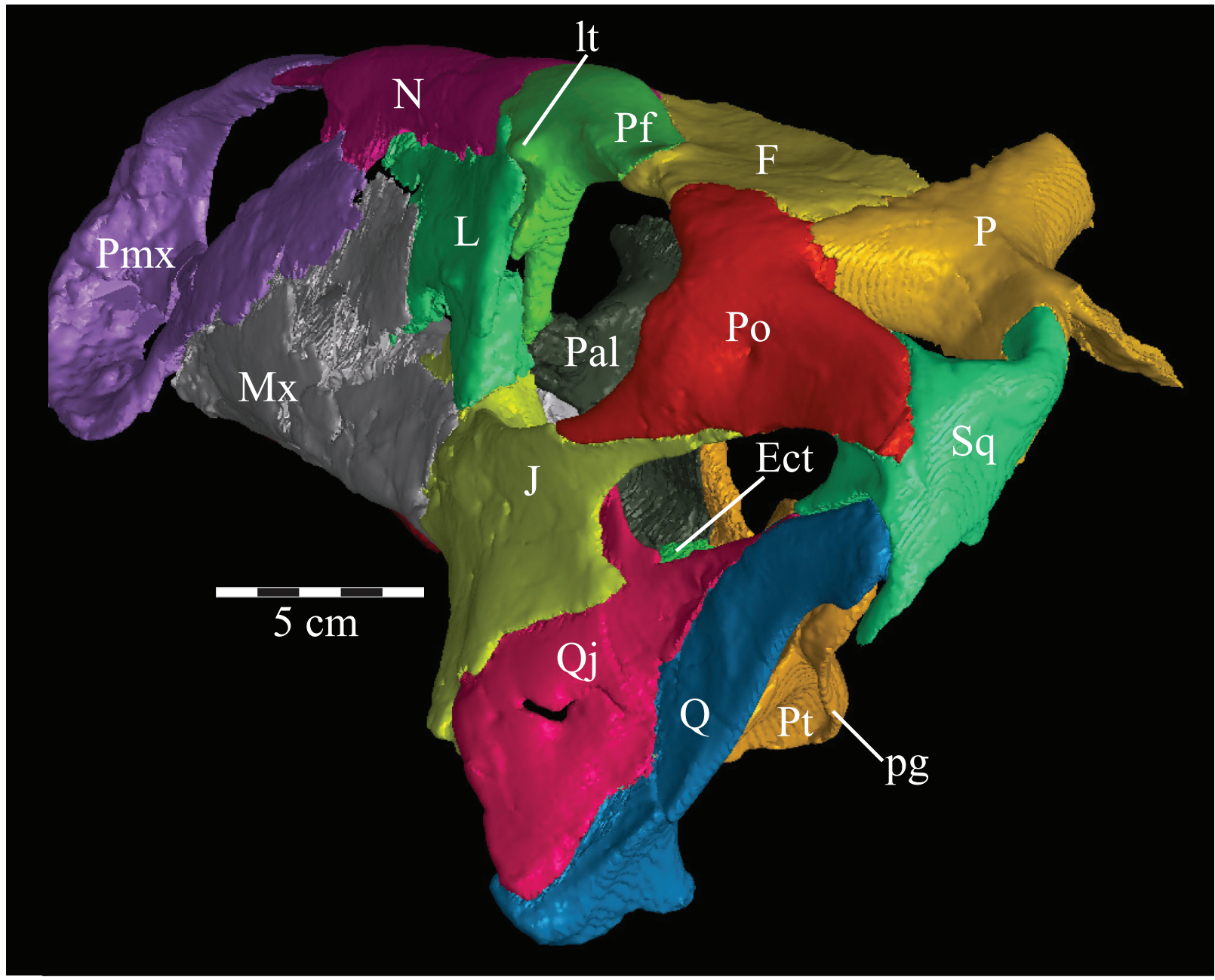

FIGURE 15. Posterolaterodorsal view of the left orbit of the virtual skull of OMNH 58340, presenting a view of the joint between the prefrontal, lacrimal, jugal, and palatine. The parallel contour lines on the posterior surfaces of the parietal, prefrontal, quadrate, and squamosal, are an artifact of the CT scanning process, and represent the faces of individual slice images. These lines occur on any surface angled near parallel with the scanner. Anterior is into the page, toward the top left. Abbreviation: It - lateral tubercle; pg - pterygoid groove.

to cover half of the pterygoid wing of the quadrate with its broad upper and lower points (Figure 1, Character 36:0). Galton (1974a, figure 8) figured a very similar shape for Hypsilophodon foxii. In some iguanodontians (e.g., Mantellisaurus atherfieldensis, Iguanodon bernissartensis) more derived than Theiophytalia kerri (Figure 7), the upper and lower points of the quadratic process become much more divergent. The quadratic process is almost entirely covered by the wing of the quadrate in anterior aspect. It is possible that this simple overlapping joint allowed posterolateral slipping of the latter element across the pterygoid. The basipterygoid process of the basisphenoid occupies the deep furrow between the medial and quadratic processes of the pterygoid in a peg-and-socket joint (Figure 1; see description of the basisphenoid below). This furrow is bordered ventrally by a strong ridge running from the middle of the medial process out along the lower edge of the quadratic process (Figure 1).

Nasal. In OMNH 58340, the elongate left nasal is preserved in articulation with the skull, while the incomplete right nasal is preserved with the disarticulated right maxilla (Figure 3). In transverse cross section, each nasal forms a dorsally convex arch that deepens slightly from the end of the naris to the orbit.

The anterior nasal forms less than a quarter of the border of the naris with two small, pointed processes (Figures 2, 18, Character 11:0), as reported for Tenontosaurus dossi (Winkler et al., 1997) and Ouranosaurus nigeriensis (Taquet, 1976). The lower of these processes does not extend as far 


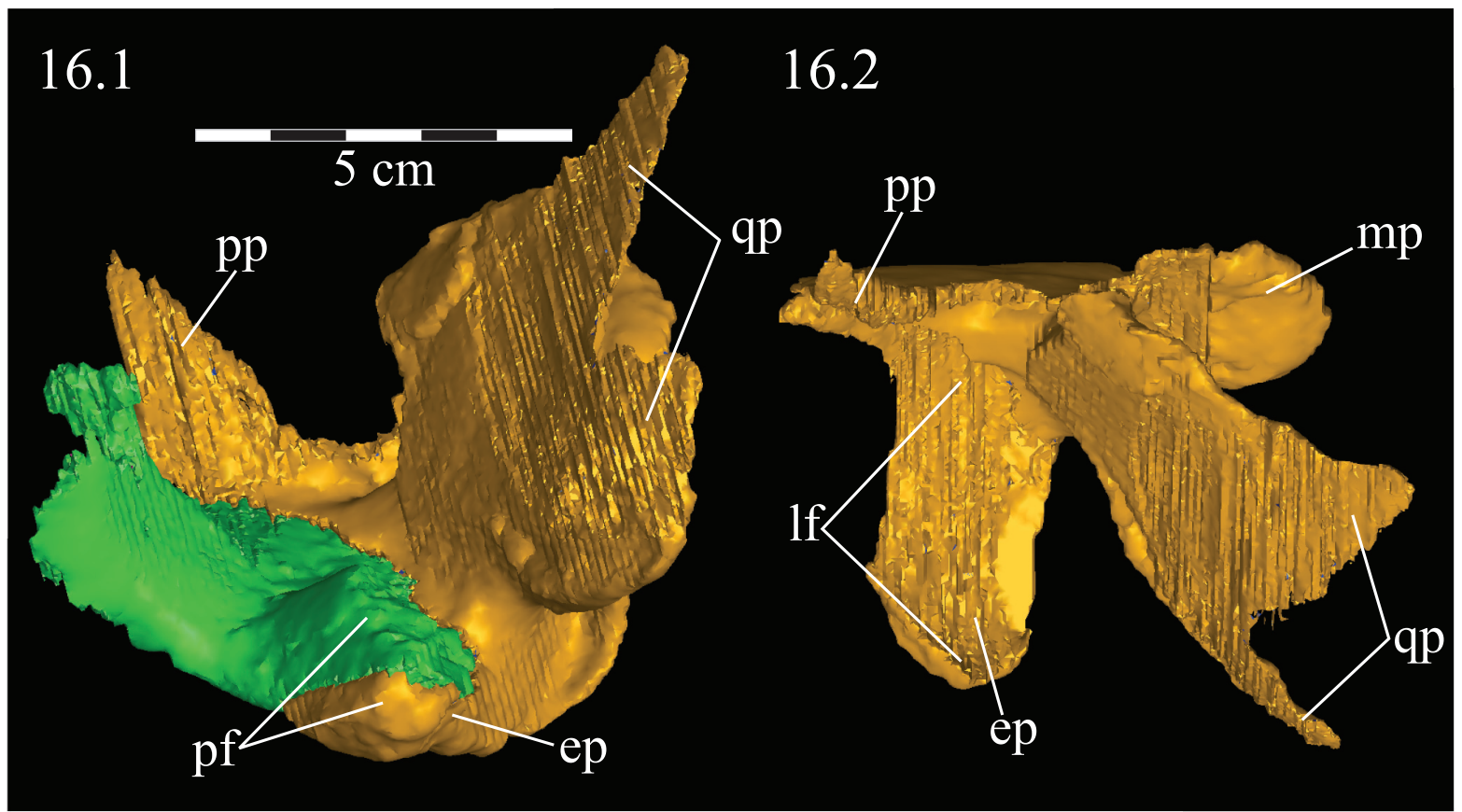

FIGURE 16. Lateral view (16.1) of the virtual ectopterygoid (green) and pterygoid (yellow) of OMNH 58340. Dorsal view (16.2) of the virtual pterygoid only. Anterior is to the left. Abbreviations: ep - ectopterygoid process; If - lateral fossa; pf - pterygoid flange; pp - palatine process; mp - medial process; qp - quadratic process.

forward as the upper (Figure 2). Beginning along the upper process, the nasal is met medially by the nasal process of the premaxilla for about two-thirds of their shared border. The premaxilla then lies in an open groove along the medial edge of the nasal, preventing contact between the paired nasals for more than half of their length (Figures 5, 18, Character 13:1). The nasals may have bordered each other in the sinusoid line preserved behind their articulation with the premaxillae (Figure 5), possibly alternately overlapping in a manner similar to Hypsilophodon foxii (Galton, 1974a), but the disarticulation of the right side of the rostrum creates uncertainty here. Below this, a gentle, dorsally convex arch runs back along the medial surface of the nasal to the end of the contact between that element with the premaxilla (Figure 19). Weishampel et al. (2003) suggested a similar feature in Zalmoxes robustus to be the bony base of a turbinate.

The front of the nasal is overlapped by the maxillary process of the premaxilla (Figure 2). Just behind this border with the premaxilla, the nasal is apparently excluded from contact with the maxilla by the upper anterior process of the lacrimal (Character 1:1; contra Ostrom, 1970), although this is uncertain due to taphonomic distortion and poor preservation of the extremely thin bone. The lack of nasal-maxillary contact is a characteristic shared by nearly all iguanodontians and hadrosaurs (e.g., Mantellisaurus atherfieldensis, Edmontosaurus regalis). Tenontosaurus dossi is one notable exception (Winkler et al., 1997).

The nasal continues to abut the lacrimal for about half of the length of the latter and is then laterally overlapped by it, at which point the nasal is covered above by the prefrontal (Figure 5, 10, 20, 21, Character 35:0). Just before its posterior end, the nasal overlaps the frontal (Character 12:0).

Toward the frontal, the medial border of the nasal leaves a significant gap along the midline (Figure 5, denoted by dashed line). It is possible that this gap was filled by pieces of the nasals that are now missing, or that the frontals, which are thin at this point, extended forward between the two nasals. Therefore, it is impossible to determine whether the nasals terminated in a medial point as in Zalmoxes robustus or a straight border as in Mantellisaurus atherfieldensis and Iguanodon bernissartensis, or were split by a median point formed by the frontals as in Hypsilophodon foxii.

Maxilla. The maxilla is the largest element in the skull, contributing most of the rostrum, and contains the upper teeth (Figure 2). It possesses two main components: a facial lamina connected ventrally to a dental ridge (Figures 17, 22). 


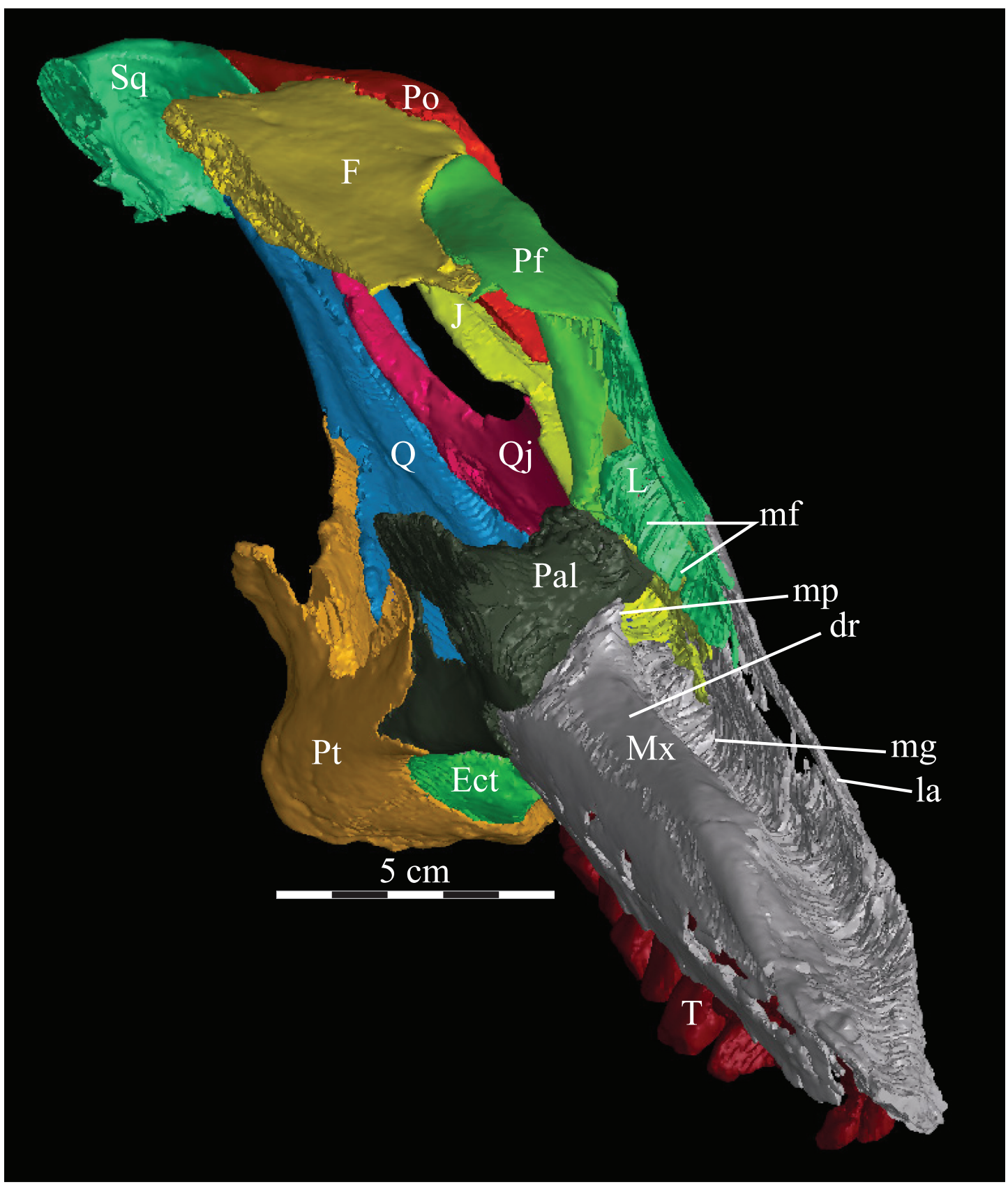

FIGURE 17. Anteromediodorsal view of the virtual skull of OMNH 58340, showing the features surrounding the maxillary groove of the maxilla, including the dental ridge and the possibility of a soft-tissue roof covering it in life. Abbreviations: $\mathrm{dr}$ - dental ridge of the maxilla; la - lamina of the maxilla; $\mathrm{mf}$ - medial flange of the lacrimal; mg - maxillary groove; $\mathrm{mp}$ - maxillary projection.

The facial lamina is triangular in lateral aspect, almost uniformly thin, and perforated behind by the front half of the antorbital fenestra (Figure 2, see descriptions of the lacrimal and jugal below). Below the fenestra, the lamina overlaps the anterior process of the jugal (Figure 14, Characters $32: 0,33: 0)$ and nearly touches the pedicle of the lacrimal (Figure 19). Above the fenestra, the lam- ina abuts the lower anterior process of the latter element. In most iguanodontians and hadrosaurs more derived than and including Theiophytalia kerri (Figure 7), the jugal abuts the maxilla laterally, often via a lateral process, much farther forward (e.g., Iguanodon bernissartensis, Edmontosaurus regalis). 

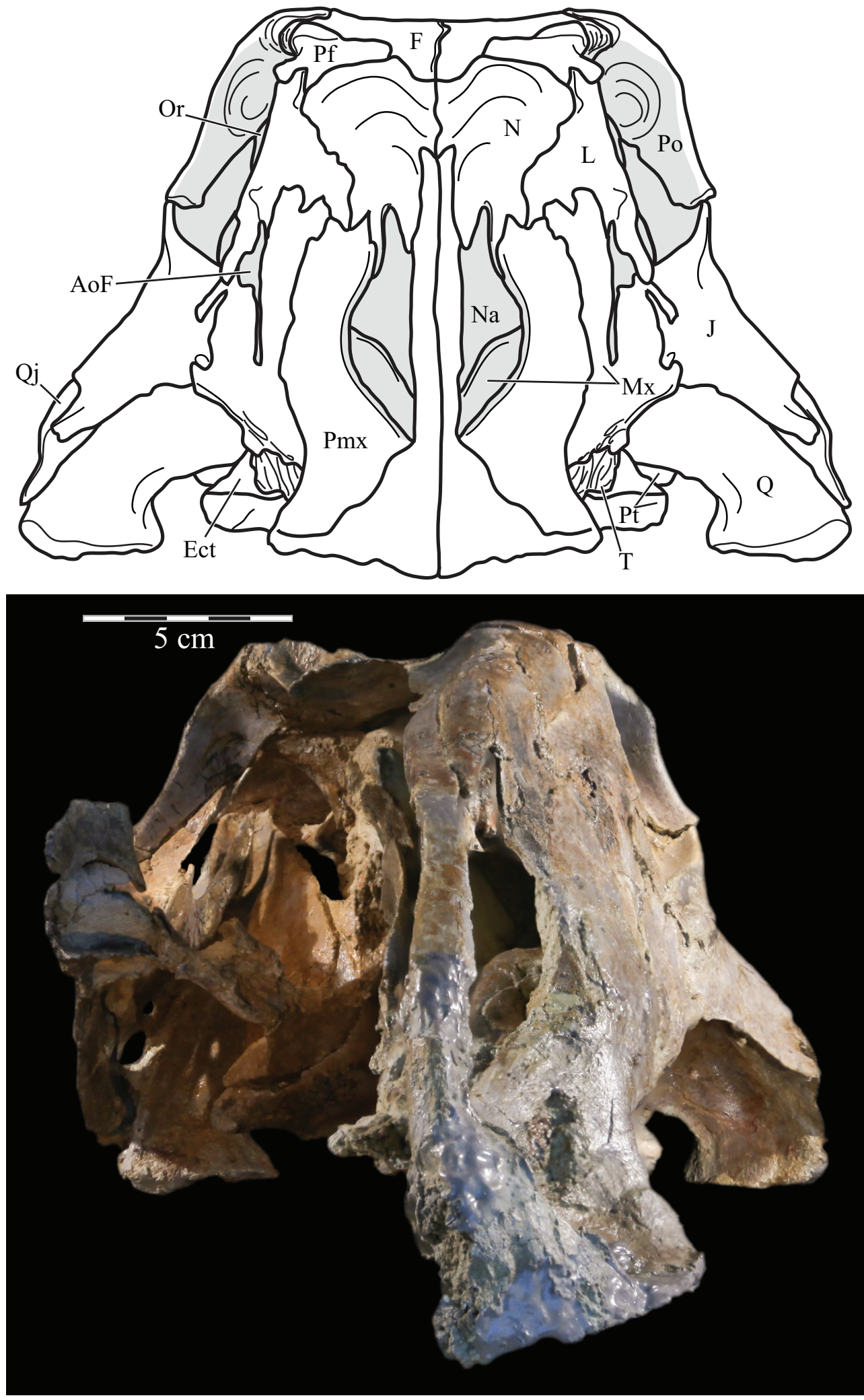

FIGURE 18. Anterior skull schematic (above) and photograph (below) of OMNH 58340. The two images are set to the same scale, demonstrating the amount of displacement in the right side of the skull. The schematic was reconstructed by digitally mirroring the left side of the rostrum and suspensorium in order to approximate the actual appearance of the skull. Natural fenestrae are shaded gray. Anterior is out of the page. 


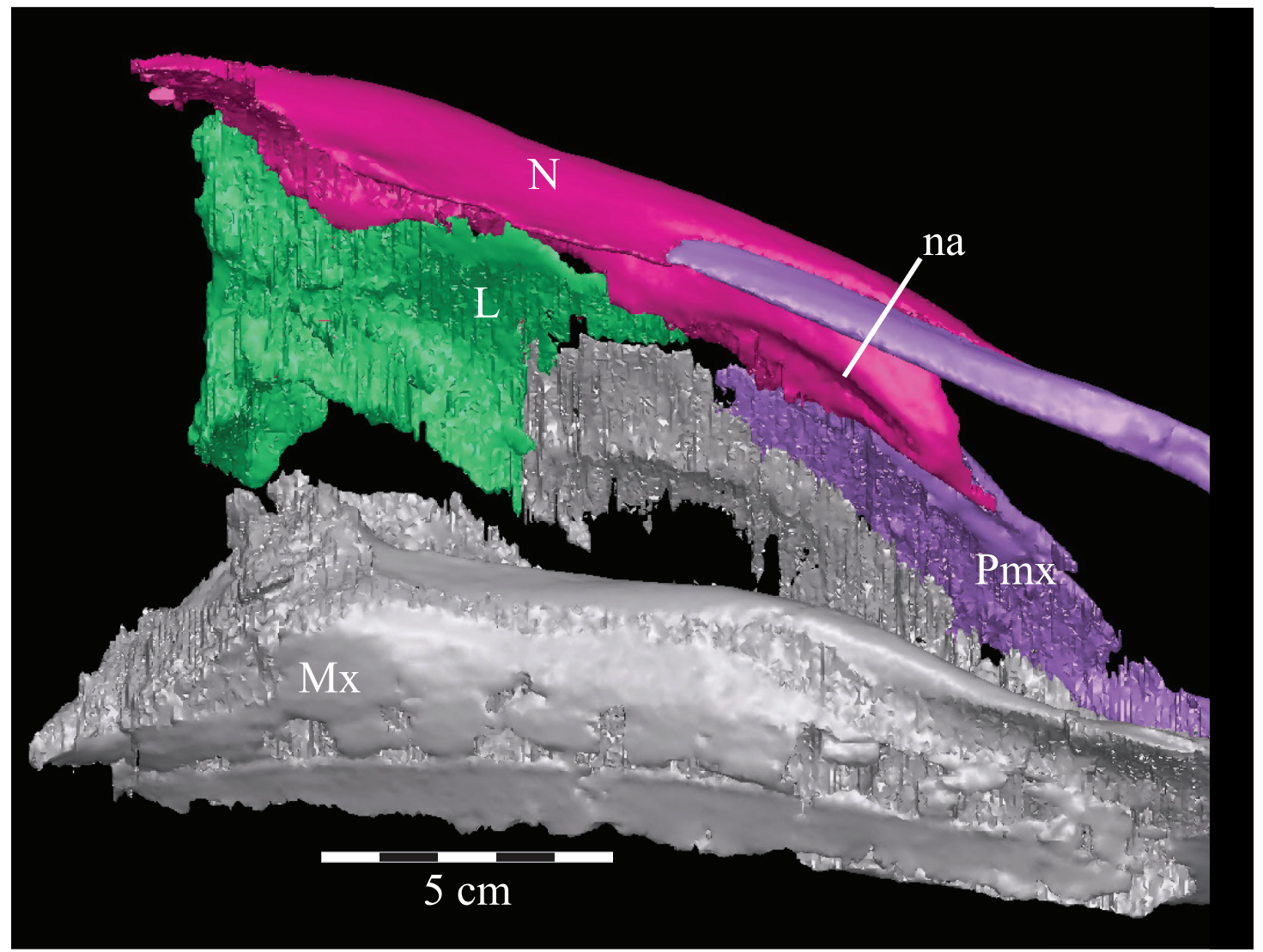

FIGURE 19. Medial view of the virtual left premaxilla, nasal, lacrimal, and maxilla of OMNH 58340 showing the nasal arch of the nasal just below its articulation with the nasal process of the premaxilla. Anterior is to the right. Abbreviation: na - nasal arch.

Above, the lamina is excluded from contact with the nasal by the anterior process of the lacrimal behind, which it overlaps, and in front by the maxillary process of the premaxilla, which overlaps it (Figure 2, Character 1:1). Although the maxilla apparently lacks direct contact with the nasal internally, a state which may be due to preservation, externally the premaxilla and lacrimal clearly divide the former elements. In front, the root of the maxillary process of the premaxilla overhangs the large maxillary foramen (Figure 2).

Below, as in most ornithopods, the lamina curves sharply inward toward the tooth row, forming a buccal ridge (Figures 2, 22, 23.1). The underside of this ridge is perforated by a number of foramina, ranging from $1 \mathrm{~mm}$ to $5 \mathrm{~mm}$. These continue up into the bone and open into the maxillary canal between the ridge and the lamina (Figure 22).

The maxillary canal begins as a blind opening in the anterior process of the jugal and continues forward as a deep and wide groove (Figure 22), narrowing and shallowing, until it is covered by a lateral extension of the dental ridge. The front of this canal becomes labyrinthine and very difficult to follow, possibly diverging and converging several times. Continuing through this network, however, is a single canal that runs forward from the main groove to open in the maxillary foramen.

In front of the maxillary foramen, where the premaxilla overlaps maxilla, the facial lamina continues to shrink in height and begins to run inward until it joins the flattening dental ridge (Figure 17). The two pieces of the maxilla converge as the element interjects between the plate of the vomer below and the beak of the premaxilla above (Figure 8 ), and the maxilla narrows to a single pointed anterior process (Figure 17). Basal ornithopods have a single anteromedial process extending from the maxilla, while more derived forms (Figure 7, e.g., Protohadros byrdi and Edmontosaurus regalis) have two.

The dental ridge of the maxilla is wide along its length (to accommodate the teeth) and is covered dorsally in a fine texture, resembling wood grain (Figure 11.2). The dorsal surface of the ridge 


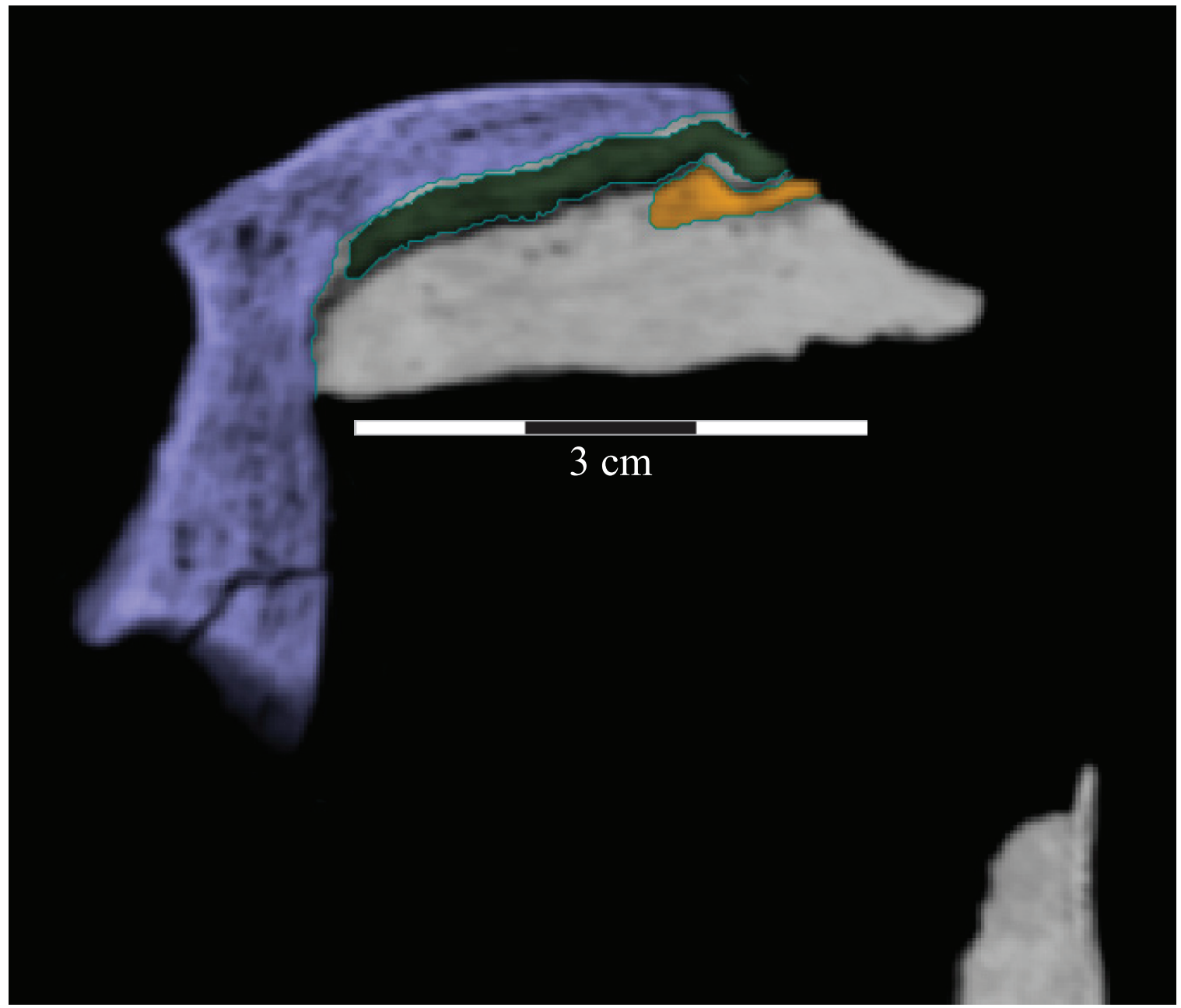

FIGURE 20. Coronal CT image, taken at the anterior edge of the left orbit, of the prefrontal (violet), nasal (dark green), and frontal (yellow) of OMNH 58340 showing their articulation. Faint green lines are an artifact of the program used to define the element on each image. Anterior is into the page.

is angled upward for the front half of its length (Figure 10.2). At the center, the ridge levels off and continues back until it sends a lateral projection up, under the lateral process of the palatine (Figure 17). The ridge then slopes quickly down to end in a medially oriented process that articulates with the ectopterygoid (Figures 9, 10.2). Lateral to this process, the dental ridge abuts the ectopterygoid and jugal (Figure 14).

There is an axial ridge that runs along the back half of the dorsolateral edge of the dental ridge (Figure 17). Below this ridge on the outside of the dental ridge, there is a gap in the lamellar bone, exposing the cancellous bone beneath (Figure 22). The lamellar bone at the edges of this gap is cusped, and may have continued outward in life. Interestingly, there is a corresponding ridge on the medial side of the facial lamina above. This, together with the preservation of a roof over the anterior maxillary canal, suggests that there was a cartilaginous or poorly ossified lamina which continued behind, possibly continuous with the medial flange of the lacrimal (see description of the lacrimal below), to the lateral process of the palatine (Figure 17). This may correspond to the medial of the two dorsal processes described by Galton (1974a) for Hypsilophodon foxii. It is also possible, however, that these features correspond to the similar feature on the nasal (described above), and may be the remnants of unpreserved turbinates.

On the medial side of the dental ridge is a dorsally arched sulcus that runs the length of the alveolar trench and separates the bulk of the maxilla from the alveolar parapet below (Figure 9). The sulcus is perforated by a foramen for each tooth family. A pattern in the relative sizes of the foramina is unclear due to preservation, but it is possible that they increase and decrease in size, relative to 


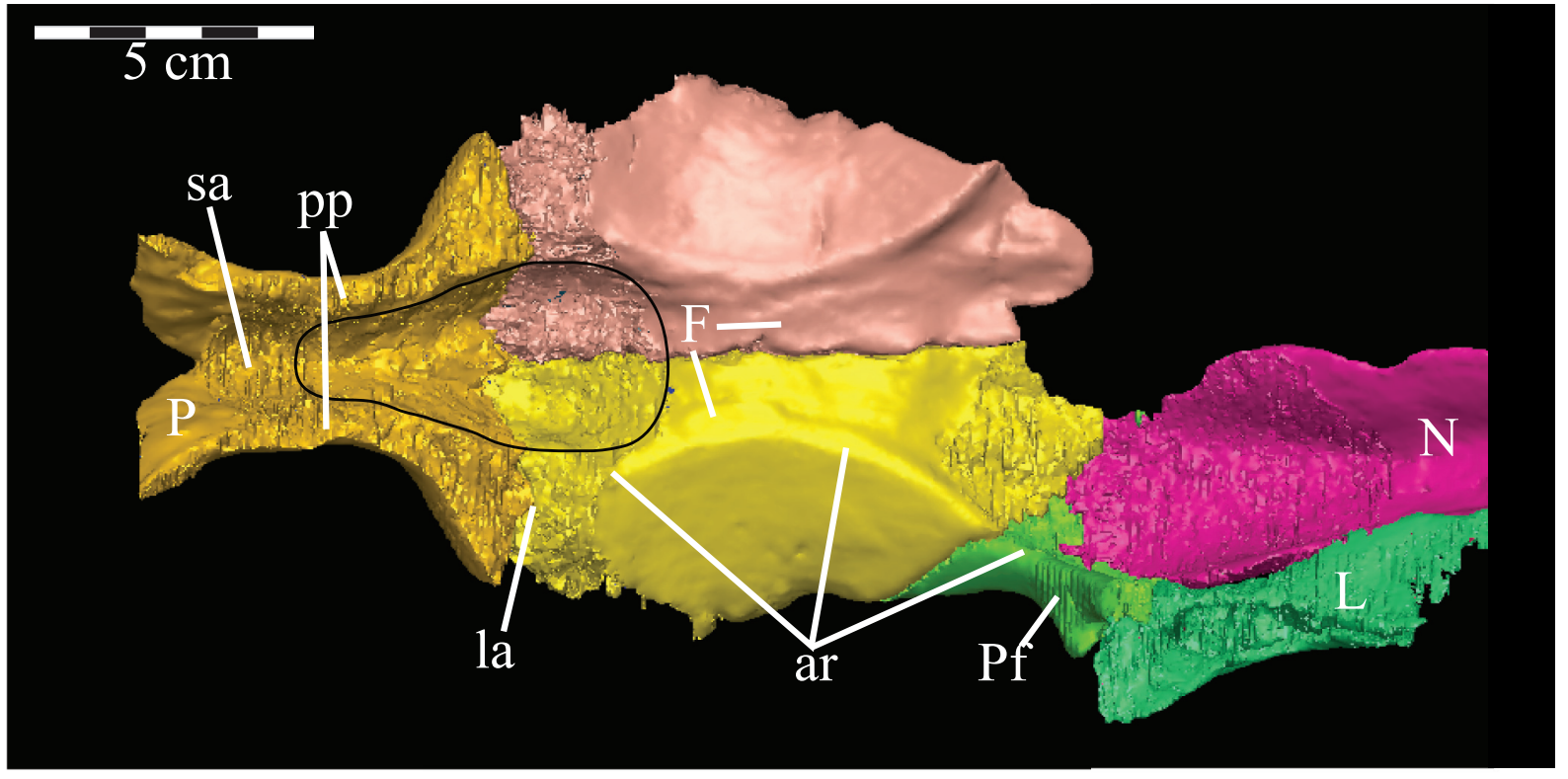

FIGURE 21. Ventral view of the skull roof of OMNH 58340. The palate and the lateral facial elements have been removed to allow for the viewing of the associations of the elements depicted. Location of endocast indicated by black outline. Note the nasal and prefrontal ending at nearly the same point posteriorly. Anterior is to the right. Abbreviations: la - area of articulation with capitate process of laterosphenoid; pp - pedicles of partietal; ar - arcuate ridge; sa area of articulation with supraoccipital.

the size of the teeth in their various positions, as in hadrosaurs (Ostrom, 1961). The medial surface of the parapet is covered in a distinctive, very low and fine ornamentation, which was probably associated with the physiological and metabolic requirements of growing new teeth (Norman, 1980). The same ornamentation is reproduced on the alveolar parapet of the dentary (Figure 23.2). The foramina likely allowed germ teeth to be shuttled from the parapet into the alveolar trench (Edmund, 1957). There are 13 tooth families within both maxillae of $\mathrm{OMNH}$ 58340 (Character 30:13).

Within the alveolar trench, the outline of the ventral surface of the bone is periodically indistinct and very difficult to trace. A pattern emerges between the more or less indeterminate areas of the trench, showing a series of hollows separated by ridges (Appendix 4: Maxilla). This undulating surface corresponds to areas of greater and lesser definition in the ventral surface of the bone, with clearer structure in the ridges between tooth families and more porous, less organized bone in the hollows for their roots. This pattern is echoed identically in the alveolar trench of the dentary (see description of the dentary below).

Lacrimal. Both lacrimals of OMNH 58340 are preserved fairly well, the left in articulation with the skull, and the right with the disarticulated maxilla (Figure 3 ). The lacrimal is laminate and roughly tri- angular with a dorsoventrally oriented body, a ventral pedicle, and a primary upper anterior process ending in a point (Figure 2). A shorter lower anterior process obscures the triangular profile (Figure 2 ). Although the element is fairly flat, a slight lateral convexity extends back from the front tip of the lower anterior process. The lacrimals extend upward nearly to the level of the skull roof, a relative height that is reflected in few, if any, reconstructions of ornithopods. This may represent an artifact of preservation (e.g. Dryosaurus altus), possibly due to transverse compression and the resultant heightening of the skull. Ostrom (1970, p. 87 , figure 8 ) extensively noted a tall rostrum as a principal character of Tenontosaurus tilletti.

The upper anterior process of the lacrimal partially overlaps the maxilla, preventing that element from making a clear external contact with the nasal (Figure 2, Character 1:1, see descriptions of the maxilla and nasal above). While the anterior tip of the process is close enough to the maxillary process of the premaxilla, preservation of the thin bone in this area is poor enough to make contact between the two debatable. A similar forward extension of the lacrimal is seen in basal iguanodontians, such as Tenontosaurus dossi (Winkler et al., 1997) and basal ornithopods, although the element may not contact the premaxilla in all cases. In contrast, the maxillary process of the premaxilla in 


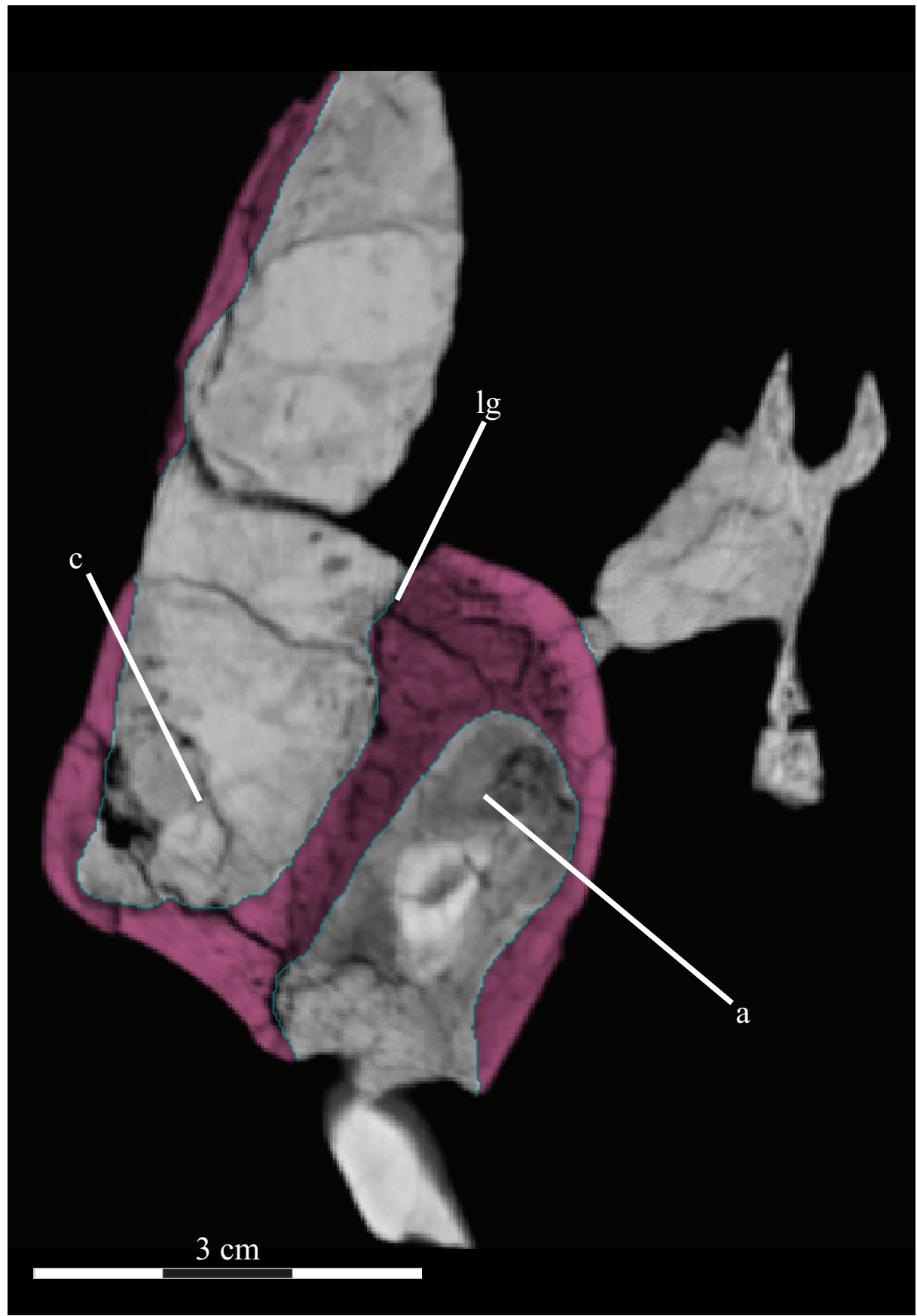

FIGURE 22. Coronal CT image of the left maxilla (pink) of OMNH 58340, taken midway along the axial length of the element, showing its facial lamina (left) and dental ridge (right, surrounding two teeth, lighter, due to higher density). Faint green lines are an artifact of the program used to define the element on each image. Abbreviations: a - alveolus; c - canal; Ig - lamellar gap.

hadrosaurs and iguanodontians more derived than and including Theiophytalia kerri (Figure 7) extends farther back across the apex of the maxilla (e.g., Iguanodon bernissartensis, Edmontosaurus regalis).

The lacrimal alternately abuts, overlaps, and is overlapped by the nasal above (Figures 2, 5, 15,
Character 28:0). Behind this, the lacrimal very broadly interlocks with the prefrontal in a sigmoid curve (Figure 2). A similar contact is seen in Ouranosaurus nigeriensis (Taquet, 1976). The posterior border is nearly vertical and, halfway down, begins to expand into a pedicle (Figure 15), which forms the posterior border of the antorbital fenestra (Fig- 


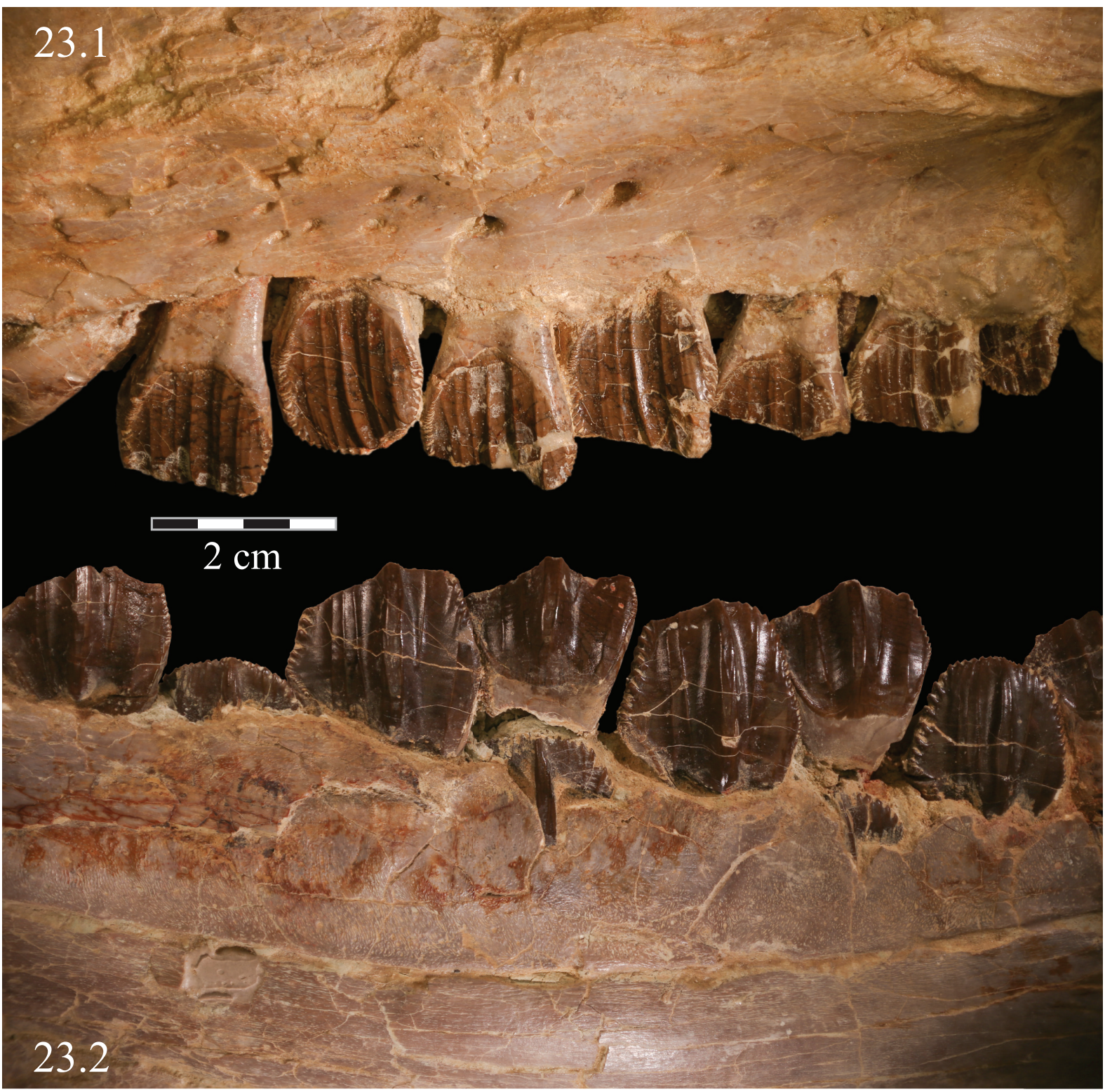

FIGURE 23. Labial photograph of the left maxillary dentition (23.1) and lingual photograph of the left dentary dentition (23.2) of OMNH 58340. Note the finely ornamented surface of the alveolar parapet of the dentary between the lower exposed surfaces of the teeth and the dorsally arched sulcus.

ure 2). This pedicle overlaps the jugal below and meets the lateral process of the palatine in a digitate suture (Figures 15, 17). The keyhole-shaped antorbital fenestra runs diagonally down at about a 45-degree angle to the tooth row (Figure 2). Just below the point where the pedicle begins, there are two small tubercles on the lateral surface of the lacrimal (Figures 2, 15).

The medial side of the lacrimal continues the expansion of the pedicle forward to the tip of the lower anterior process in the form of a dorsally arched flange (Figures 17, 24). This flange of the lower anterior process runs along the upper border of the antorbital fenestra and appears to be taken up by a similar ridge on the medial surface of the maxilla (Figure 10.2). In OMNH 16562 (which is transversely compressed) Winkler et al. (1997) described the antorbital fenestra as reniform and suggested that it is dorsally bordered by a fossa. Upon further review, however, this fossa appears to be the ventral surface of the medial flange, which can also be seen in the specimen figured by Ostrom (1970, YPM 5456). This flange and its continuation on the maxilla may also represent the bony base of a turbinate (see descriptions of nasal and maxilla above). 


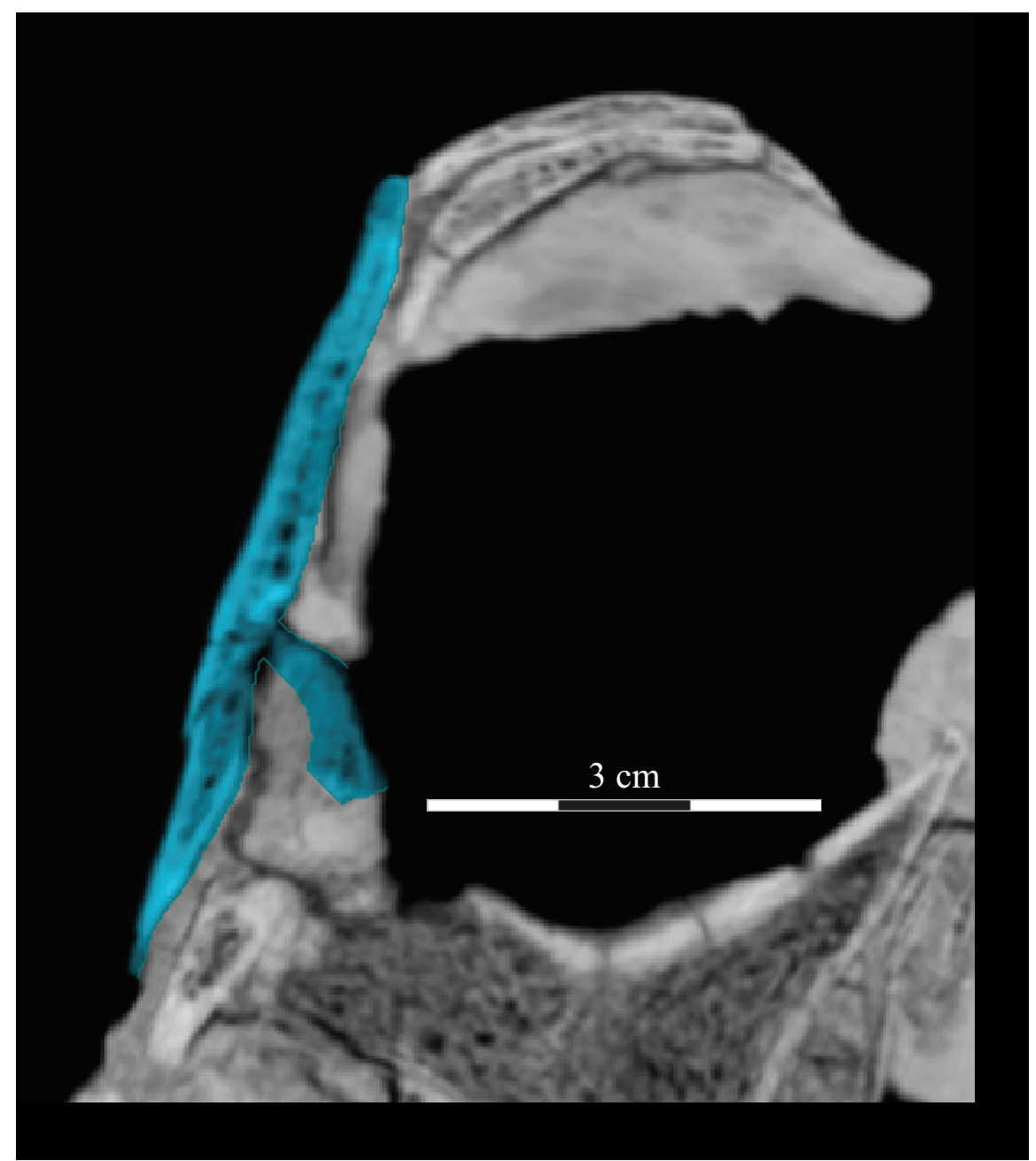

FIGURE 24. Coronal CT image, taken just anterior of the left orbit, of the lacrimal (light blue) and its shelf (lower right) of $\mathrm{OMNH}$ 58340. Faint green lines are an artifact of the program used to define the element on each image. Anterior is into the page.

The lacrimal forms the outside of the anterior border of the orbit (Figure 15, Character 27:1). Together with the prefrontal, the lacrimal articulated with the palpebral, which is not preserved in this specimen. In addition, a lacrimal canal does not appear to be preserved within the lacrimal, as is the case in some other ornithopods (e.g. Hypsilophodon foxii, Edmontosaurus regalis), although there is a foramen visible beneath the articular surface for the palpebral between the lacrimal and prefrontal (Figure 15). The canal opens above the flange described above, but its anterior extent is unclear.

Prefrontal. The prefrontals are composed of two major elements, subequal in length: a horizontally oriented, elliptical body and a long ventral process (Figures 2, 25). Anteriorly, these form the upper corner and medial portion of the orbital border, respectively. The ventral process is triangular in cross-section with lateral and anterior and posterior medial corners (Figure 25). The process tapers under the body then flares into a small ventral pedicle (Figure 15). Both of the prefrontals of $\mathrm{OMNH}$ 58340 are preserved, the left articulated with the skull and the right completely disarticulated, in two pieces (Figure 25).

The body of the prefrontal is deepest behind and tapers forward (Figure 25). In dorsal aspect, the body is narrower and rectangular behind, and expands forward, becoming more rounded (Figure 5 ). The dorsal surface of the prefrontal is very smooth and convex at its rounded anterior end and concave behind. The body appears relatively large when compared with the condition present in most other ornithopods (e.g., Hypsilophodon foxii, Man- 


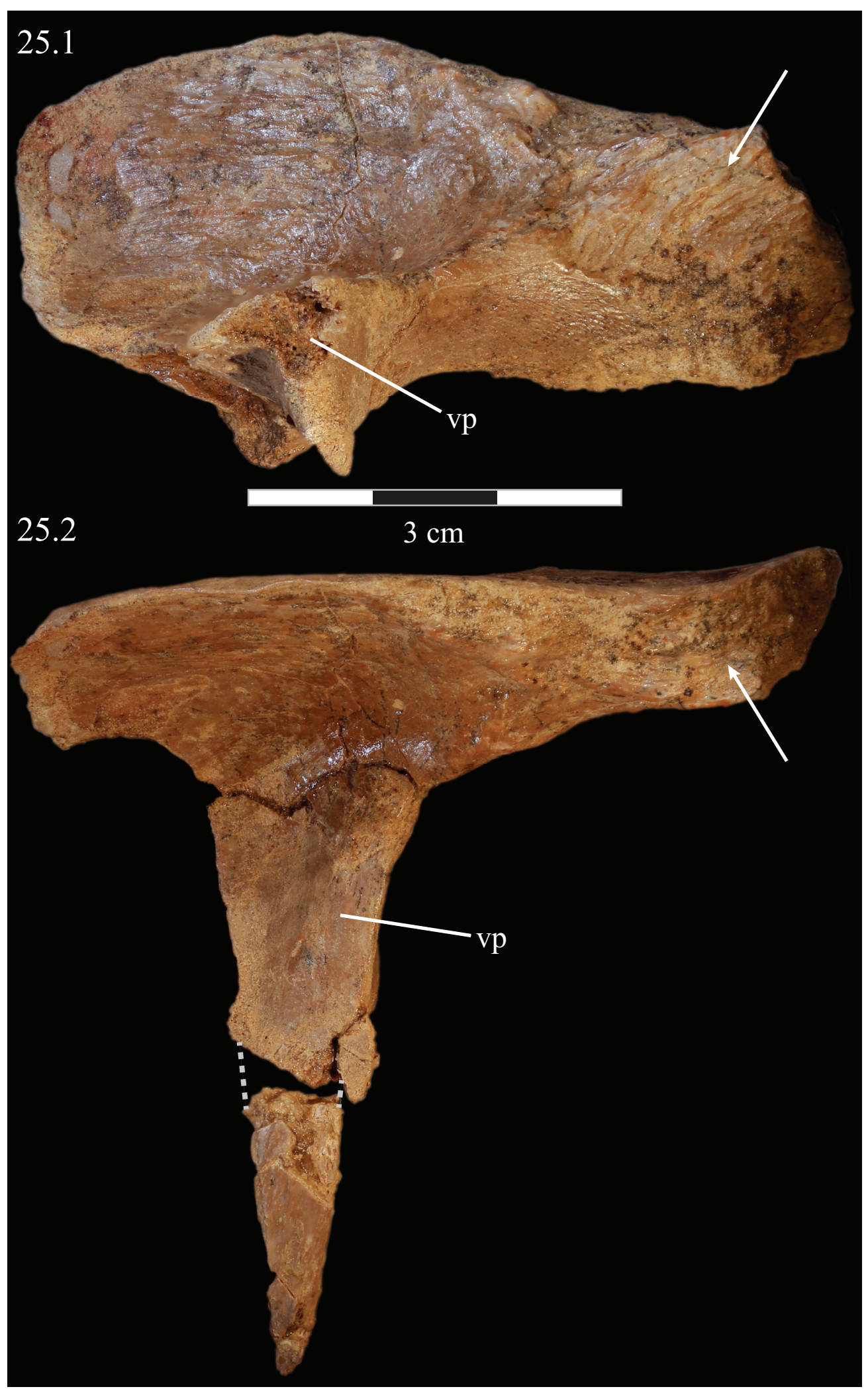

FIGURE 25. Ventral (25.1) and medial (25.2) photographs of the disarticulated right prefrontal of OMNH 58340. Anterior is left. Arrows indicate undulating texture on the ventral and medial surfaces of the element, which indicate the area of articulation with the frontal. The space between two broken ends of the ventral process in 25.2 is indicated by a dashed gray line. Abbreviation: $v p$ - ventral process. 


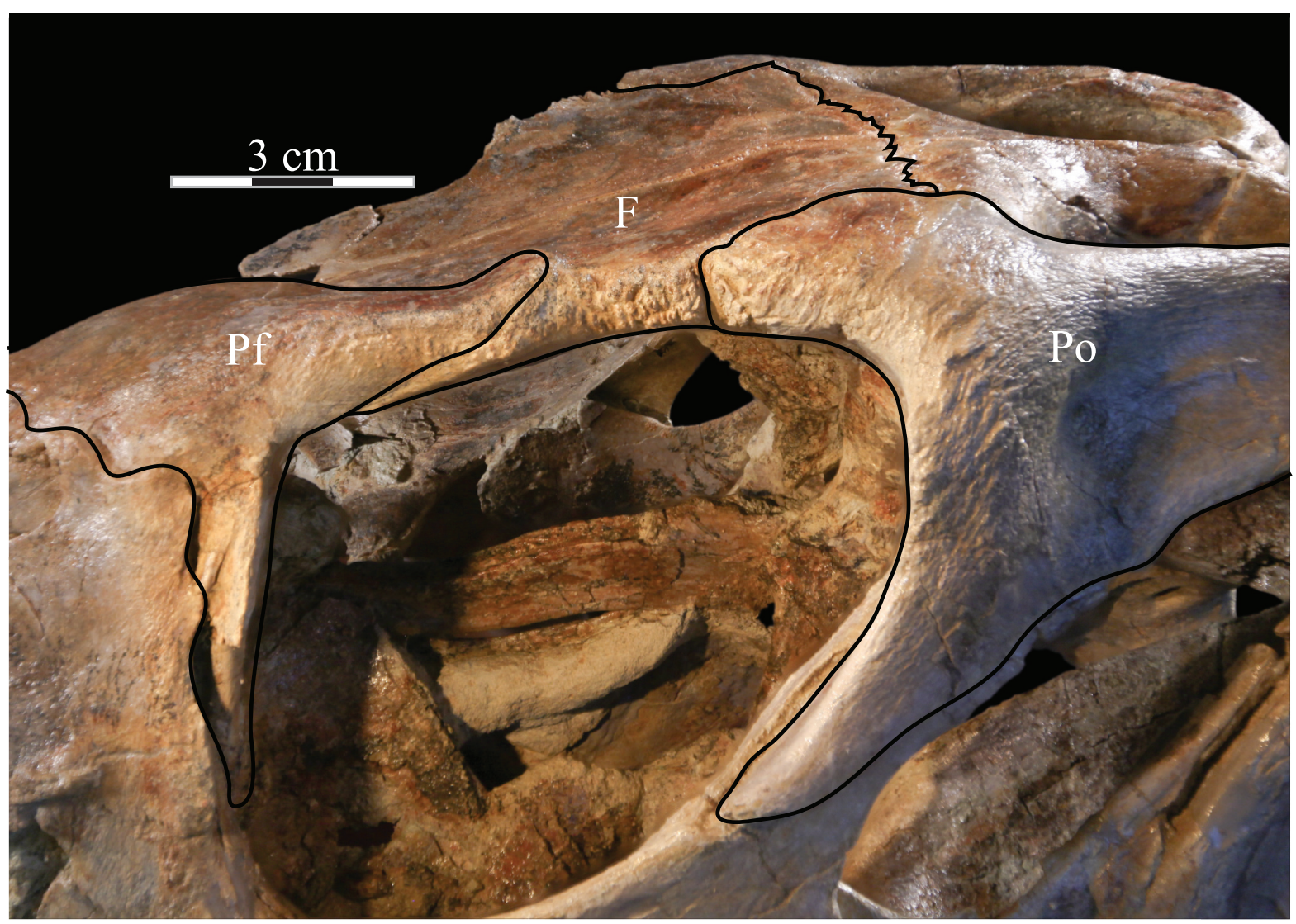

FIGURE 26. Photograph of the left prefrontal, frontal, and postorbital of OMNH 58340, showing the high degree of rugosity above the orbit. Anterior is to the left.

tellisaurus atherfieldensis), although not in comparison to Tenontosaurus dossi.

Behind the ventral process, the surface of the prefrontal above the orbit is curved and covered in increasingly rugose ornamentation (Figure 26). This rugose edge forms the front of the upper orbital border, which is completed behind by the frontal and postorbital (Figures 2, 26, see descriptions of the frontal and postorbital below). The anterior underside of the prefrontal is concave (Figure 25), allowing the element to overlap the nasal and frontal (Character 12:0). Here, the arched nasal lies immediately under the prefrontal, where it interjects between the articulation of the latter with the frontal (Figure 20, Character 35:0). The underside of the prefrontal is convex behind, with a medial corner formed by the posterior, medial, and ventral surfaces of the bone. The ventral and medial surfaces of the prefrontal are covered in a finely undulating texture which matches the texture within a fossa on the frontal, into which this corner of the prefrontal fits (Figures 25, 27).

The posterior medial corner of the ventral process continues, first dorsally as a ridge on the underside of the prefrontal, and then back to become the arcuate ridge of the frontal (Figure 21, see description of frontal below). The front of the process meets the body with a small, lateral tubercle (Figure 15). The broadly sinusoidal ventral border of this tubercle forms the lower anterior edge of the prefrontal, which articulates with the lacrimal (Figure 15).

The tapering of the ventral process is mainly transverse (Figure 15), and the pedicle extends slightly forward (Figure 10.2). Halfway down the ventral process are a pair of flanges (Figures 2, 15 ), one of which joins with the lacrimal (see description of the lacrimal above) to form an interdigitate articular surface for the palpebral, which is not preserved in OMNH 58340 (see description of the palpebral below). Here, the ventral process of the prefrontal also forms the anterior border of the orbit. The pedicle at the tip of the process interjects between the articulation of the pedicle of the lacrimal and the lateral process of the palatine (Figures $15,28)$.

The ventral process does not have any readily recognizable homologues in other ornithopods. 


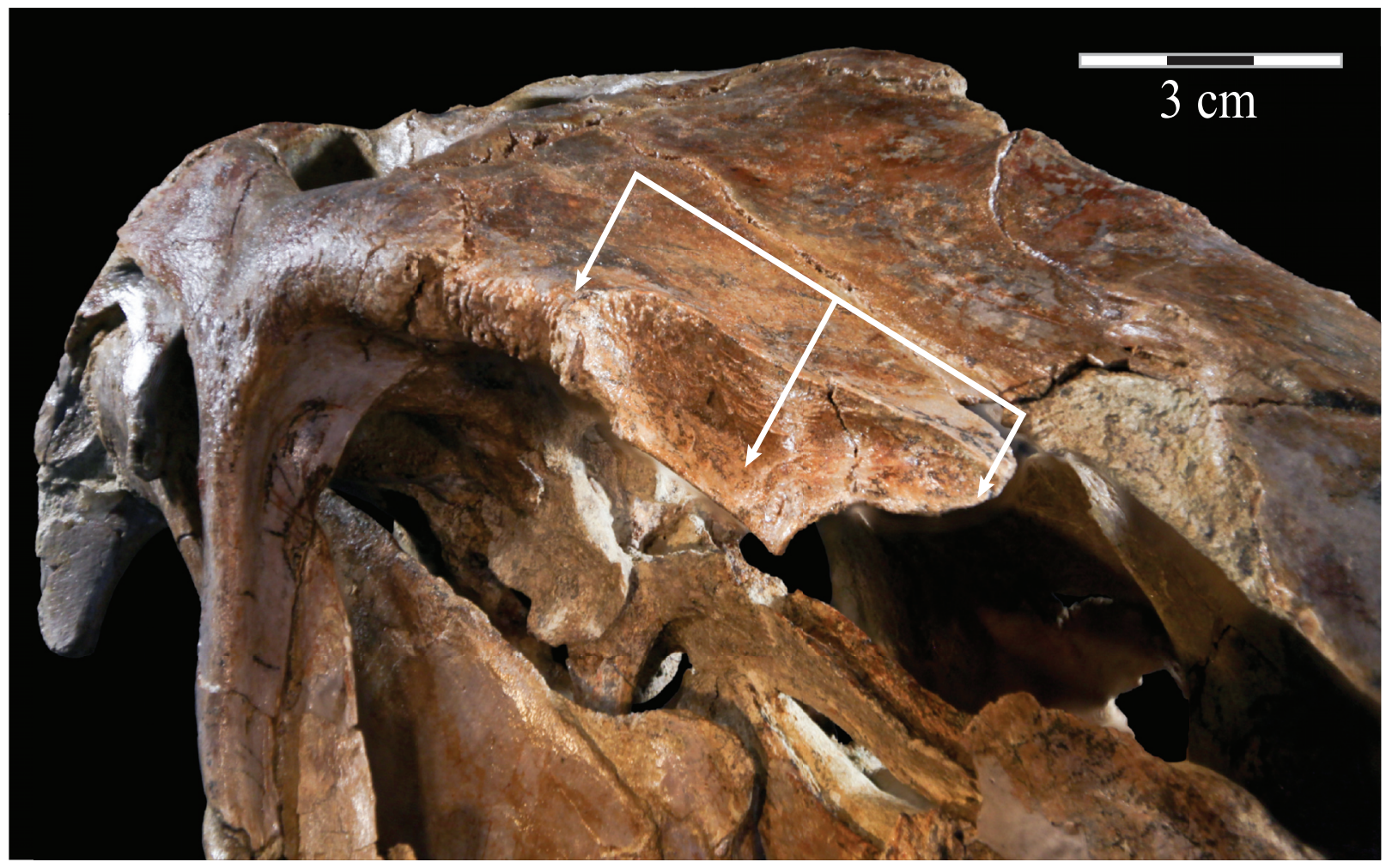

FIGURE 27. Photograph of the prefrontal fossa (indicated by arrows) of the right frontal of OMNH 58340. The pyramidal corner of the disarticulated prefrontal fits into this fossa. Anterior is to the right and out of the page.

The only exception may be the 'lateral sheet' of Galton (1974a, p. 35) in Hypsilophodon foxii, although this is only briefly described.

Palpebral. The palpebrals of OMNH 58340 are not preserved. However, another specimen, $\mathrm{OMNH}$ 16562, retains this element. This specimen is transversely flattened and only the right side of the skull has been prepared, which is remarkably complete, although distorted.

Although present, the palpebral is not particularly well preserved, and is displaced ventrally and laterally from what appear to be the articular surfaces of the prefrontal and lacrimal, respectively. The element is elongated and flares at its posterior end. The articular end appears to be expanded although, due to poor preservation, it is difficult to discern. The palpebral is thin, similar to the element described by Ostrom (1970). There is no articular surface on the frontal or postorbital for the posterior tip of the palpebral.

Frontal. The frontals are a pair of flat bones that appear hexagonal in dorsal aspect and which articulate in a weakly interdigitate border along the midline of the skull (Figures 5, 9). Both frontals are preserved articulated and intact in OMNH 58340. The combined frontals are approximately as wide as they are long among iguanodontians basal to Theiophytalia kerri (Figure 7, Character 6:1). The frontals of basal ornithopods are generally more elongate (e.g., Hypsilophodon foxii and Parksosaurus warreni) and those of iguanodontians more derived than and including T. kerri, including hadrosaurs, tend to be wider and shorter (e.g., Mantellisaurus atherfieldensis, Edmontosaurus regalis).

The frontal is dorsally concave, forming a shallow fossa running down from the highest points at the lateral and posterior borders (Figures 26, 27). The ventral surface of the element is very smooth. There is an outwardly concave arcuate ridge that continues forward and inward from the parietal to end on the prefrontal (Figure 21). The space between the paired ridges was probably occupied by olfactory epithelium. The arch of the laminate orbitosphenoid fits between the ridges posteriorly, forming the anterior border of the braincase. The frontal quickly tapers on either side of this ridge, likely accommodating the eye laterally and associated ocular and olfactory structures medially. Lateral to the posterior end of the arcuate ridge, the frontal smoothly abuts the laterosphenoid below (Figures 9 (schematic), 21, Character 15:0). 


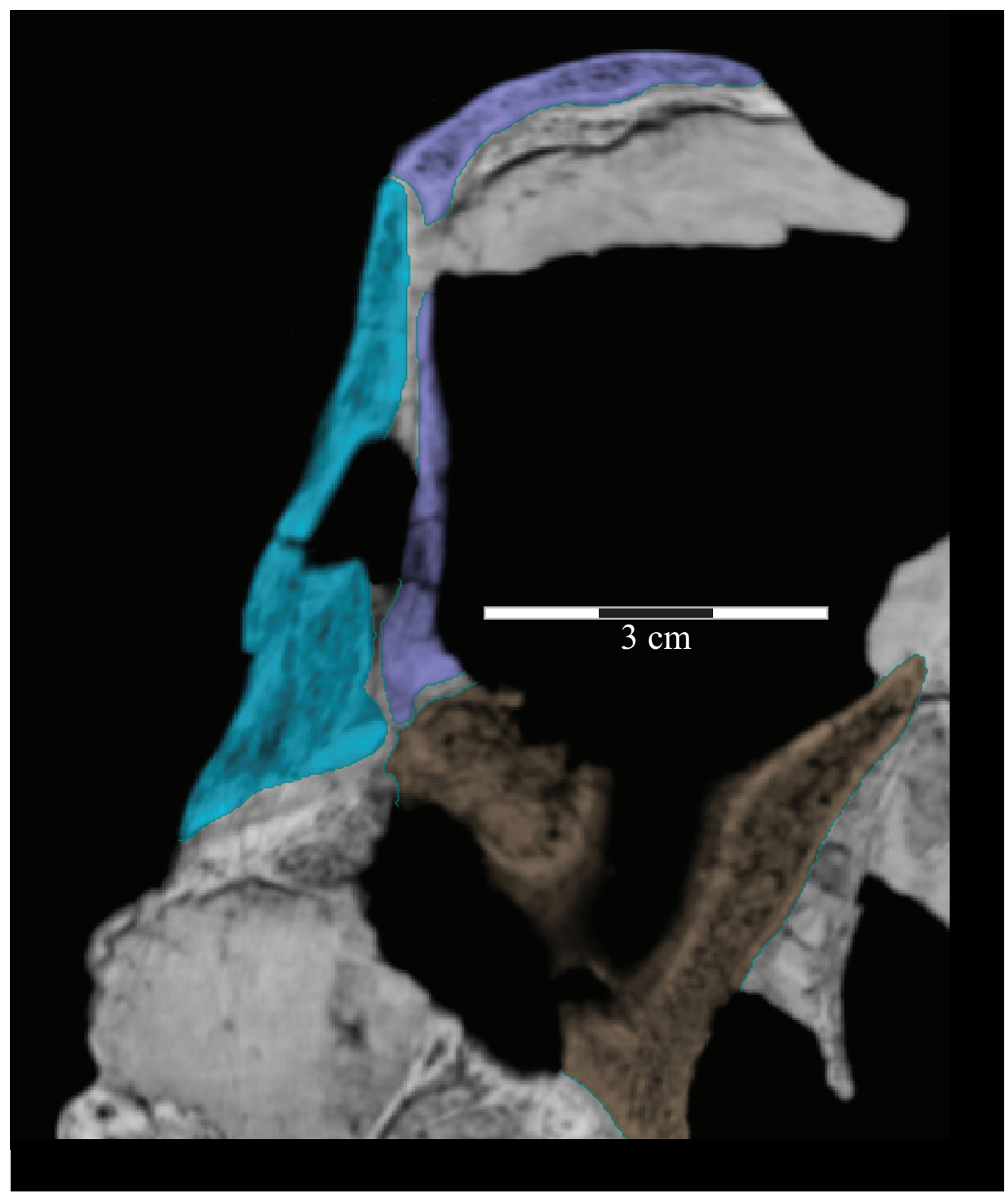

FIGURE 28. Coronal CT image, taken just anterior of the orbit, of the left prefrontal and its ventral process (violet), lacrimal (light blue), and palatine and its lateral process (brown) of OMNH 58340 showing the nature of their articulation. Faint green lines are an artifact of the program used to define the element on each image. Anterior is into the page.

There are a few, very small foramina on the ventral side of the frontals as well, the bores of which are too small to be observed in the CT images gathered. Of note, there is a short, shallow furrow, running outward on the underside of the right frontal, just beyond the arcuate ridge. The feature contains matrix, and its edges are smooth enough that it does not appear to be an artifact of preparation. In addition, the articulated nature of the skull and postcranial skeleton indicates that this individual was not a victim of predation or scavenging. It is possible that the mark was made when the right side of the skull was taphonomically shifted and the maxilla disarticulated.

The frontal appears wedge-shaped in medial aspect, tapering forward (Figure 9). As it is preserved, the medial edge of the bone in front of its median suture angles laterally, coming to a point against the medial edge of the prefrontal (Figure 5, photograph). This is restored as a straight, dotted line in the schematic, as neither the nasal nor the frontal are preserved here (see description of the nasal above). 
The straight posterior border of the frontal forms a strongly interdigitate suture across the entire face of the parietal as well as the superior orbital process of the postorbital (Figure 5, Character $7: 1)$. The articulation with the postorbital continues onto the lateral surface of the frontal. The element bears deeply rugose ornamentation where it is laterally exposed as part of the upper border of the orbit (Figure 26). The frontal forms less than half of the dorsal rim of the orbit (Character 8:1), similar to nearly all iguanodontians, including hadrosaurs (e.g., Tenontosaurus dossi, Mantellisaurus atherfieldensis, and Edmontosaurus regalis), with few exceptions (e.g., Dryosaurus altus, Jinzhousaurus yangi), much less than the contribution of the element to the orbital border in basal ornithopods (e.g., Hypsilophodon foxii and Parksosaurus warreni). The lateral surface of the frontal is cut by a deep fossa where it articulates with the prefrontal (Figures 2, 27). At the anterior tip of the fossa, the rear edge of the nasal interjected between the frontal and the prefrontal (Figure 20).

Postorbital. The postorbitals are a pair of triradiate bones, composed of superior and inferior orbital processes extending forward and a squamosal process that extends posteriorly (Figures 2, 5). Both postorbitals are preserved articulated in OMNH 58340.

The inferior orbital process is wedge-shaped in lateral aspect, triangular in cross section, and gently sweeps forward at its lower tip where it covers the dorsal surface of the postorbital process of the jugal in a scarf joint, as in all ornithopods (Figure 2). Between the inferior and superior orbital processes, there is a deep concavity at the posterior corner of the orbit (Figure 9). Above this concavity there is a fine rugosity, continuing back from the prefrontal and frontal, which lessens until it fades away before the body of the postorbital (Figure 26, Character 16:1). This texture is present in many iguanodontians basal to Theiophytalia kerri (Figure 7, e.g., Zalmoxes robustus and Cumnoria prestwichii).

The superior orbital process is rounded dorsally. This convexity continues down along the outside edge of the inferior orbital process and back along the upper edge of the squamosal process (Figures 2, 5). There is a tubercle present on the lateral surface of the postorbital between the orbital processes that may have served as a point of attachment for musculature of the eye (Figure 15).

The squamosal process is narrow and tall with subparallel upper and lower borders and slopes outward (Figures 2, 5, 15). Beginning in a concav- ity just behind the tubercle mentioned above, striations run along the outer surface of the entire process (Figures 2, 29).

The squamosal process forms the upper part of the outer border of the superior temporal fenestra before the former lies in a scarf joint with the postorbital process of the squamosal (Figures 2, $5)$. The squamosal process roofs a lateral concavity between the postorbital and quadrate processes of the squamosal (Figures 2, 30), which was probably the origin of $M$. adductor externus superficialis (Ostrom, 1961). Finally, the lower edge of the squamosal process forms a small part of the dorsal border of the inferior temporal fenestra (Figure 2).

The large medial surface of the superior orbital process is strongly digitate and articulates with the frontal in front, the parietal behind, and the capitate process of the laterosphenoid below (Figure 5 , Characters 15:0, 17:0). This medial surface terminates in a point, behind which the postorbital is scooped into a concavity to form the outer corner of the superior temporal fenestra (Figure 5). The rest of the medial surface of the postorbital is scooped into a gentle concavity. This concavity is filled by the postorbital process of the jugal along the inferior orbital process and by the postorbital process of the squamosal along the squamosal process.

Jugal. The laminate jugal is roughly triangular in lateral aspect, and comprises a maxillary process, a small posterior tab, and postorbital and ventral processes (Figure 2). The element is laterally convex except for a small groove running under the postorbital process. In OMNH 58340, both jugals are preserved in articulation with the rest of the skull, although the right is shifted with the quadrate and quadratojugal (Figures 1, 9, 18). Among other ornithopods, the element most closely resembles that of Hypsilophodon foxii (Galton, 1974a).

The maxillary process and the front half of the jugal carry a canal, continuing back from the maxillary canal (see description of the maxilla above). The exact nature of the anterior jugal is difficult to discern due to preservation, but it is clear that the facial lamina of the maxilla overlaps it and that it contributes to the antorbital fenestra (Figure 2). Here, the jugal abuts the pedicle of the lacrimal dorsally and the lateral process of the palatine medially (Figure 15). The only other ornithopod in which this contact with the lacrimal was noted is Ouranosaurus nigeriensis (Taquet, 1976). Below this, the jugal meets the bridge between the facial lamina and dental ridge of the maxilla in front and the ectopterygoid medially (Figure 6, Character 


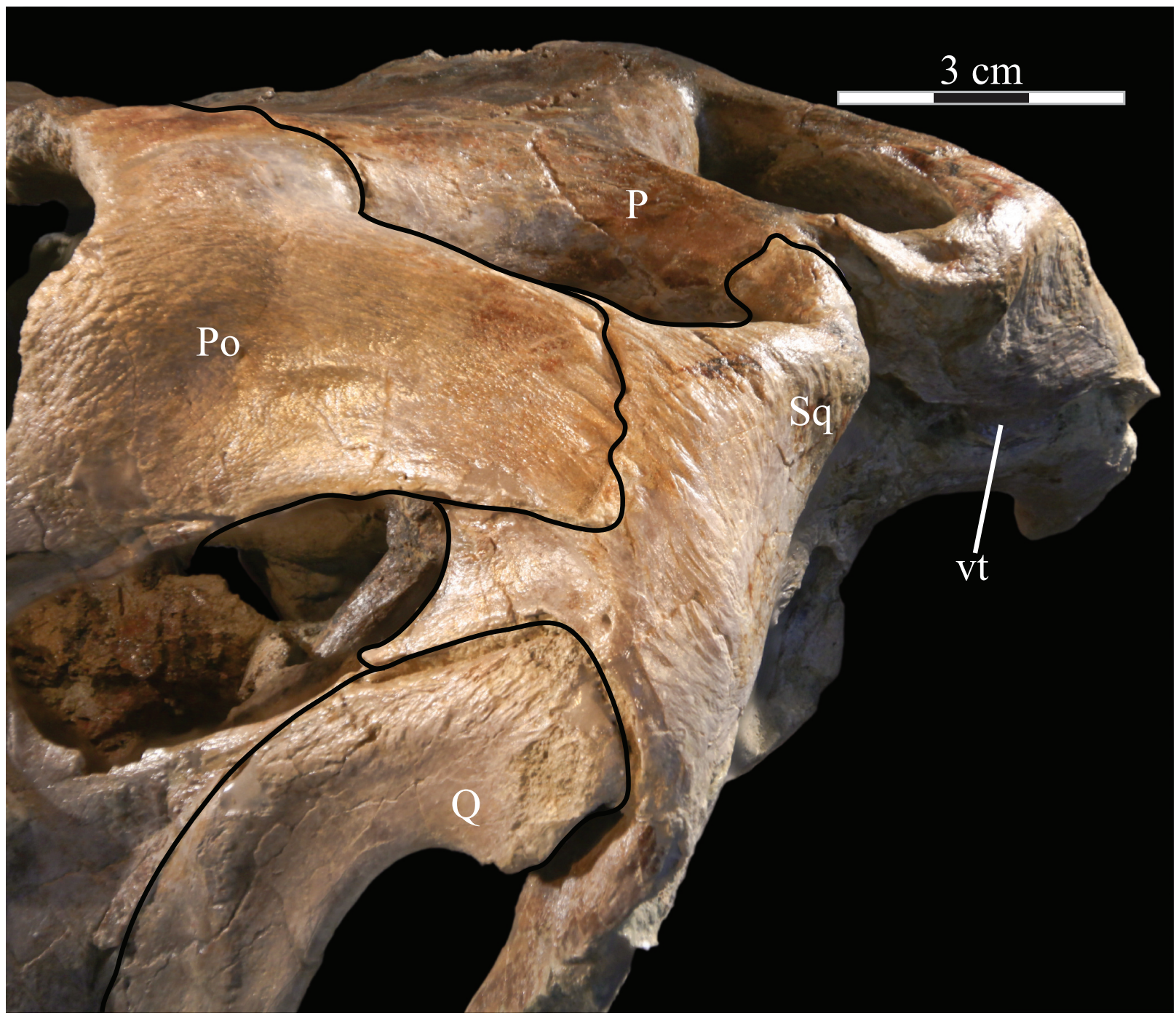

FIGURE 29. Photograph of the left squamosal of OMNH 58340, showing the deep texture on the lateral surface of the body of the bone, continued forward onto the lateral surface of the squamosal process of the postorbital. Anterior is to the upper left and into the page. Abbreviations: vt - ventral tab of the squamosal.

19:0). A medial articulation with the ectopterygoid is a plesiomorphic trait shared by all ornithopods, excluding hadrosaurs (Figure 7; Weishampel, 1984; Norman, 2004).

Behind the maxillary process on the inside of the jugal are two fossae (Appendix 4: Jugal). The lower fossa is roofed by a ridge running forward from the dorsal process of the jugal. The jugal likely contributed the outer anterior border of the adductor chamber below this ridge. The dorsal fossa is located above the ridge and forms the lower border of the orbit (Figures 2, 5, 10.2, 14).

The inferior orbital process of the postorbital overlaps the postorbital process of the jugal in a scarf joint (Figures 2, 9, 10). At its upper tip, the postorbital process meets the anterior process of the squamosal (Figure 10.2). Here, the postorbital process forms most of the anterior borders of the inferior temporal fenestra (Figures 2, 15).

Below this, the jugal divides into the larger medial and smaller lateral tabs of its ventral process (Appendix 4: Jugal). The tabs are separated by the lower edge of the anterior quadratojugal (Figure 2). The ventral border of the jugal is gently undulating and covers the coronoid process of the mandible. All ornithopods more derived than Tenontosaurus tilletti (Figure 7) feature a relatively longer quadratojugal process that, in some cases, contacts the quadrate (e.g., Zalmoxes robustus, Iguanodon bernissartensis, Edmontosaurus rega- 


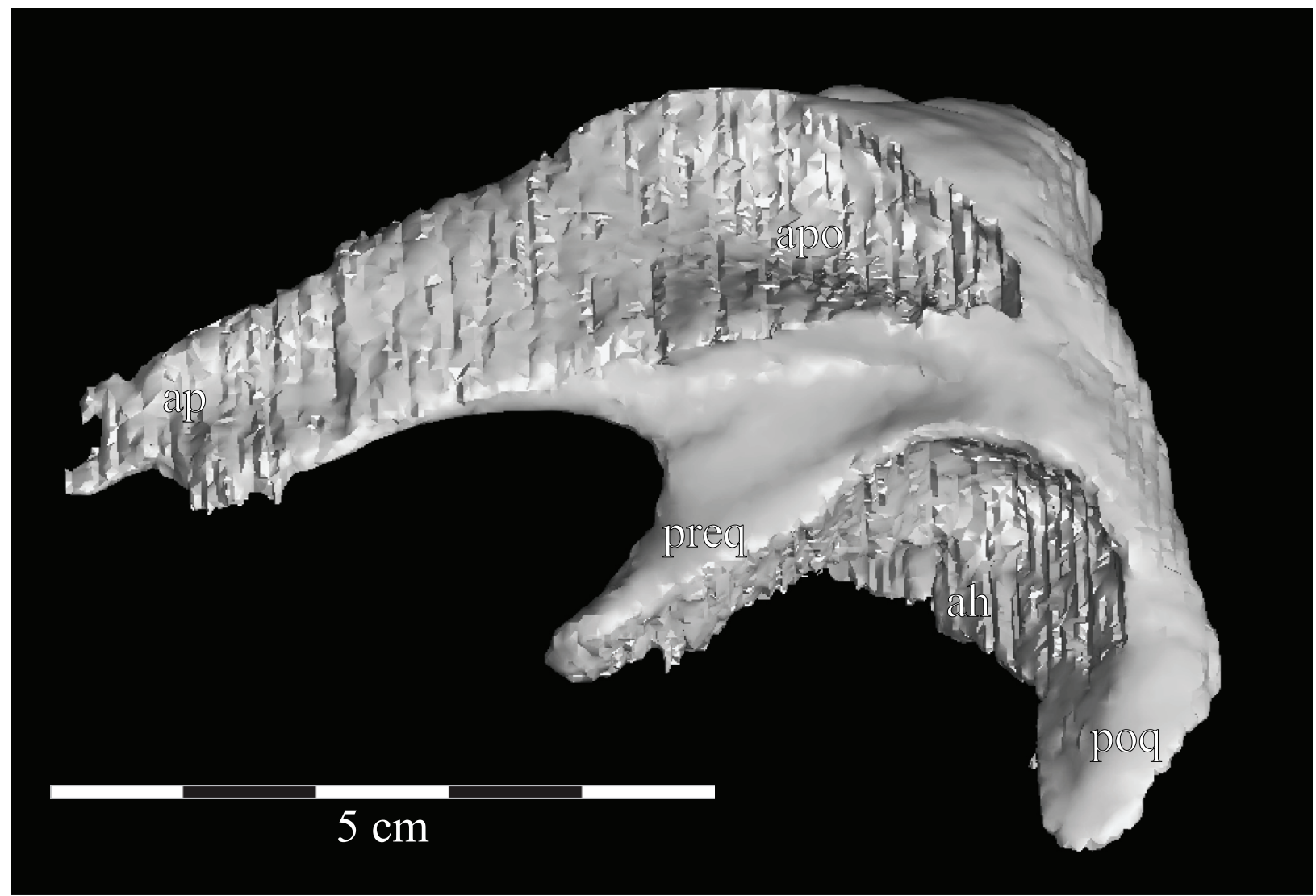

FIGURE 30. Lateral view of the virtual left squamosal of OMNH 58340 showing the deep articular fossae and the processes of the element. Anterior is to the left. Abbreviations: ap - anterior process; ah - articulation for head of quadrate; apo - articulation for postorbital; poq - postquadratic process; preq - prequadratic process.

lis). The jugal does not contact the quadrate posteriorly (Character 20:0, contra Ostrom, 1970).

Quadratojugal. The quadratojugal is a simple laminate bone, laterally convex throughout and rhomboidal in lateral aspect (Figures 2, 31). The squamosal process of the element contacts the ventral tip of the prequadratic process of the squamosal. There is a variable supplementary inferior temporal fenestra on the lower half of the element (Character 23:1). There are two subcircular fenestrae, one quite larger than the other, in the right quadratojugal of OMNH 58340 (Figure 9, photograph) and a single, slit-like one in the left (Figure 2). There also appears to be a single, large fenestra in the left side of OMNH 16562. This accessory foramen is shared by Hypsilophodon foxii and Tenontosaurus dossi. Contra Ostrom (1970, figure 8 ), the jugal does not contribute to the borders of this foramen and that element appears roughly the same length and height as the quadratojugal (Figures 2, 31, Character 26:1). The quadratojugal comprises most of the outer wall of the mandibular adductor chamber.
About half of the quadratojugal is overlapped laterally by the jugal in front and lies between the medial and lateral tabs of the ventral process of the latter (Figure 2). The squamosal process and body of the quadratojugal form the lower and posterior borders of the inferior temporal fenestra, respectively (Figure 2). The process lies in the groove between the body and the pterygoid wing of the quadrate behind, and then moves out to overlap the body, ending in a ventral point just above the articular condyle (Figures 1,2). There is no paraquadratic foramen as is found in basal iguanodontians more derived than Theiophytalia kerri (Figure 7, e.g., Mantellisaurus atherfieldensis).

Quadrate. In OMNH 58340, the left quadrate is preserved in its original orientation, running diagonally down and out from the skull roof. The right quadrate is shifted so that it appears nearly vertical from behind (Figures 1,18) and points the suspensorium dorsolaterally rather than simply forward (Figures 5, 9). When restored to their original orientation the articular condyles of the quadrates achieve the greatest width of the skull (Figures 1, 


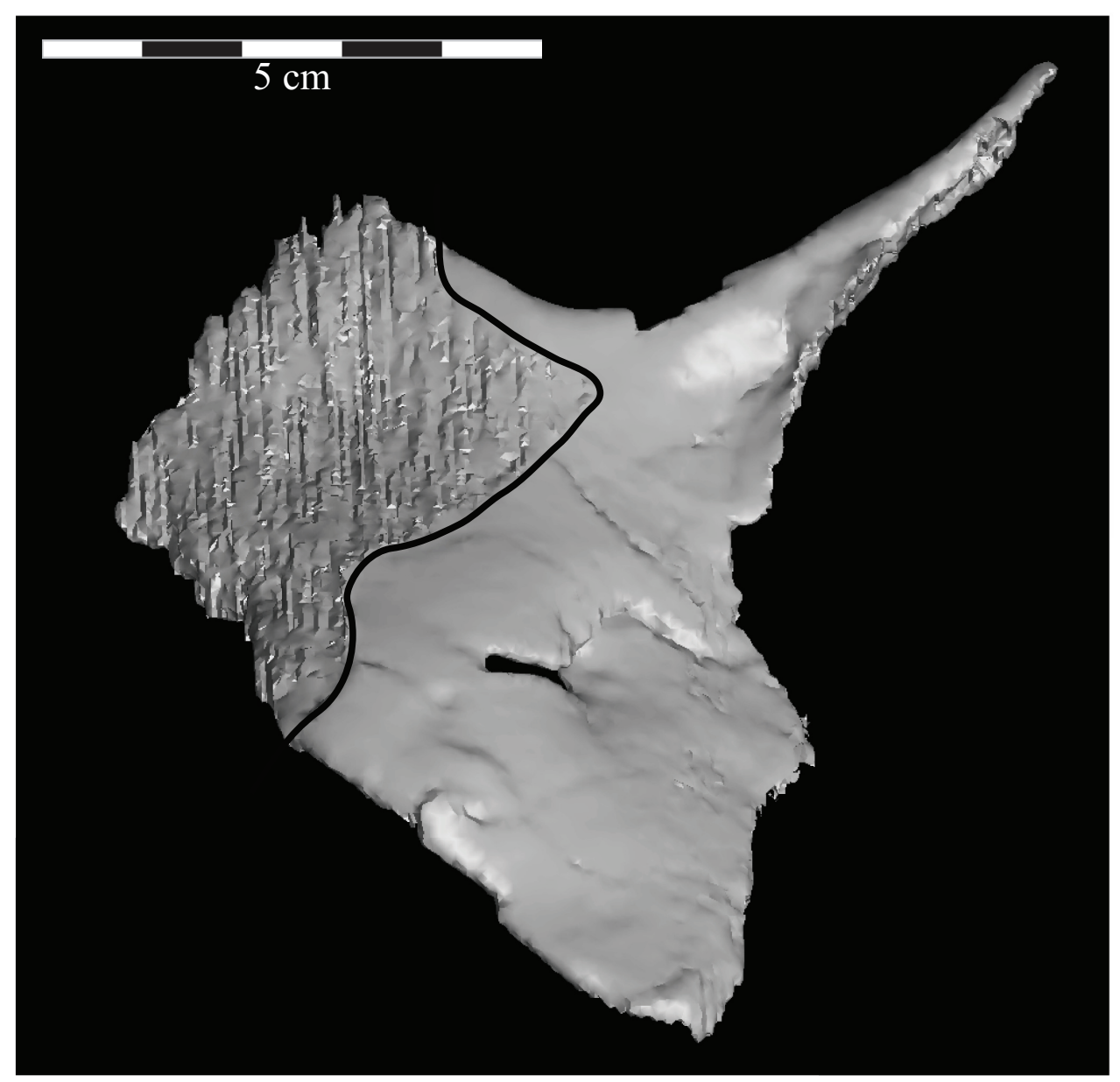

FIGURE 31. Lateral view of the virtual left quadratojugal of OMNH 58340 showing the rhomboidal shape of the element. The corrugated area on the left half of the element (outlined in black) is an artifact of the sectioning process and represents the articular surface for the jugal. Anterior is to the left.

$5,18)$, resulting in much larger jowls than restored by Ostrom (1970, figure 9), who restored the temporal region as the widest.

In lateral aspect, the quadrate is a long, fairly thin, vertical bone. When the skull is oriented at the angle indicated by the plane of the lateral semicircular canals (Figure 32), the upper body of the quadrate is angled back (Figure 2). The quadrate consists of the body, a small anterior flange, and a large pterygoid wing. This basic shape is fairly consistent throughout Ornithopoda, though it is more or less pillar-like in certain taxa (e.g., Theiophytalia kerri for a particularly curved quadrate and Protohadros byrdi for a notably straight, columnar one).

The body is very thin transversely, has subparallel borders in lateral aspect, and is overlapped by the quadratojugal (Figure 2). Halfway up, the superior process of the quadratojugal shifts to lie in the groove between the wing and anterior flange of the quadrate, and contacts the prequadratic pro- cess of the squamosal dorsally, which lies in the same groove. In Hypsilophodon foxii, there is a forward expansion of the anterior flange at this point, not present in Tenontosaurus tilletti. Also, in nearly all iguanodontians more derived than T. tilletti (Figure 7, e.g., Probactrosaurus gobiensis), there is a notch in the flange, which helps create the paraquadratic foramen (e.g., Mantellisaurus atherfieldensis, Jinzhousaurus yangi). There is no such notch in T. tilletti (Character 37:0). However, a small embayment in the flange may represent one of the early stages of the evolution of the paraquadratic foramen (Character 25:0, Appendix 4: Quadrate). The notch and foramen are lost in the hadrosaurs.

The body thins above to allow for a notch between the quadrate and the paroccipital process of the opisthotic, likely for the superficial tympanic membrane (Figure 2). Above this, the head of the quadrate is wedge-shaped in dorsal aspect (Character 39:0) and lies in a mortise created by the 


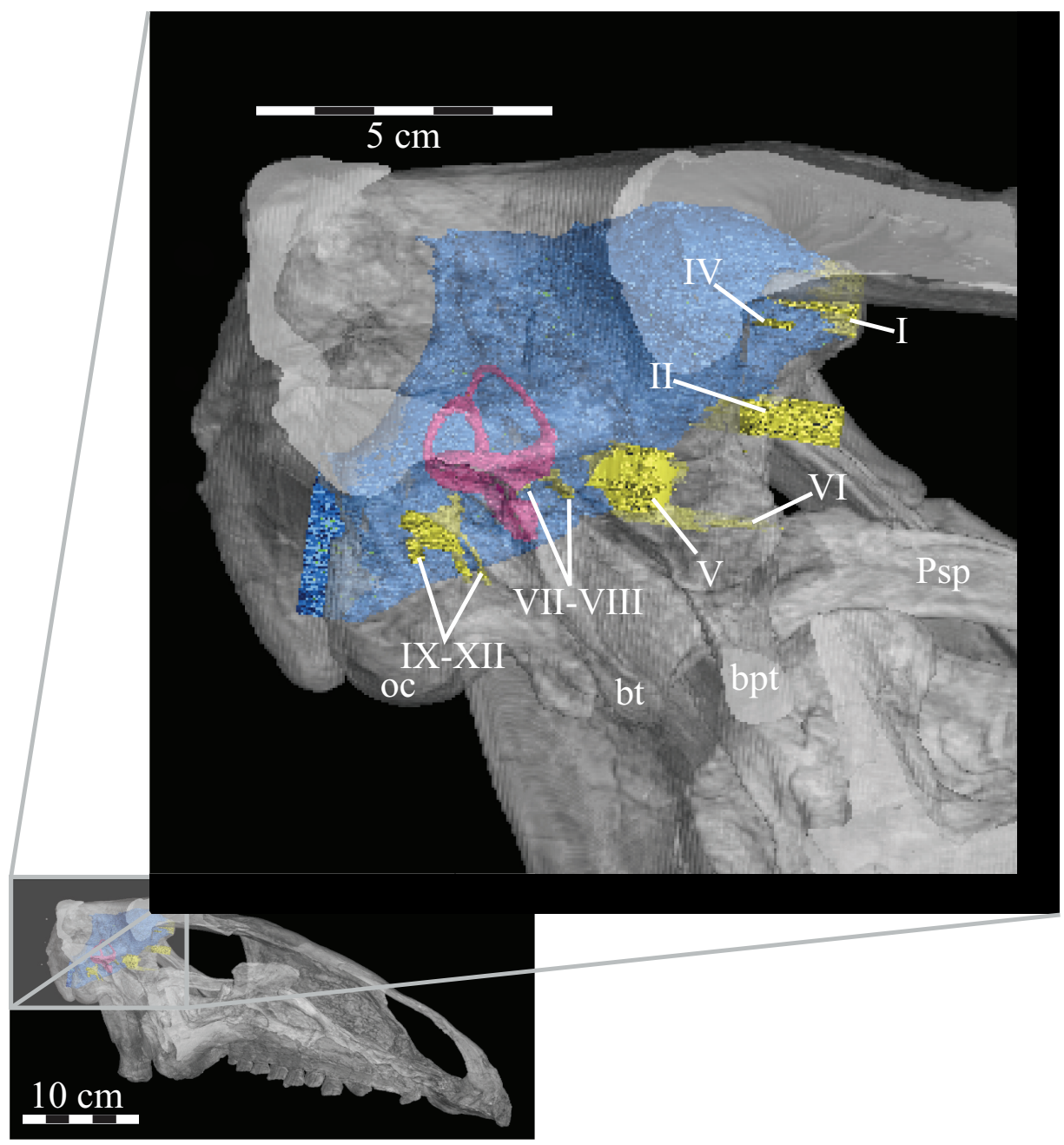

FIGURE 32. A lateral view of the virtual skull (lower left) and braincase (inlay, upper right) of OMNH 58340 with a high transparency, making the endocast (blue), cranial nerves (yellow), and semicircular canals (magenta) visible. The skull is at the 'alert position' indicated by the horizontal semicircular canals. Abbreviations: bt - basal tubera; bpt - basipterygoid process; oc - occipital condyle.

squamosal (Figures 2, 30). There is a diagonal ridge running along the outside of the body, about halfway up (Figure 2). Above and below the ridge are two very shallow fossae. The fossae appear to be sites for muscle attachments, although corresponding sites of origination or insertion of the hypothetical muscles are not immediately obvious.

The laminate pterygoid wing of the quadrate is concave behind and triangular in posterior aspect, with a rounded medial corner. The wing meets the body of the quadrate just above the articular condyle below and above at the head as it articulates with the squamosal. The convex anterior surface of the wing probably served as the origin of $M$. posterior adductor mandibulae (Ostrom, 1961). The quadratic process of the pterygoid overlaps the wing behind (Figure 1).
The quadrate expands below into the articular condyle (Figure 1, Character 38:0), which is subrectangular in ventral aspect (Figure 6), with medial and lateral tubercles (Figure 1). The larger lateral tubercle is a feature shared with Hypsilophodon foxii. In hadrosaurs, the condyle of the quadrate is narrower, effectively losing the medial tubercle (Weishampel et al., 1993). The condyle articulates with the prearticular, surangular, and articular of the mandible below.

Squamosal. The squamosal is a complex bone, composed of four processes: medial, anterior, prequadratic, and postquadratic (Figures 2, 30). Both of the squamosals are preserved in articulation in OMNH 58340. The shape of this element appears to be similar among most ornithopods. 
The medial process of the squamosal appears rhomboidal from behind, with a corner above the paroccipital process of the exoccipital and two above the supraoccipital (Figure 1). The posterior surface of the bone is concave in its middle, with striations carried back from the squamosal process of the postorbital (Figure 29). Between the ventral edge of the medial process and the paroccipital process below is a groove that begins at the top of the ascending process of the supraoccipital and runs laterally (Figure 1). There is a small tab here on the underside of the squamosal that preserves the shape of the groove but extends down to contact the exoccipital (Figures 1, 29). Medially, the squamosal curves forward to overlap the occipital process of the parietal (Figures 5,33 ) and make a small contact with the supraoccipital. The paired squamosals do not meet across the midline (Character 40:0).

The squamosal forms part of the border of the superior temporal fenestra between the medial and anterior processes (Figure 5). The anterior process of the squamosal is long, tall, and very narrow (Figure 30). The process slopes down and touches the tip of the dorsal process of the jugal in front, a feature that is either autapomorphic or yet to be described in other taxa (no character coded). Laterally, it is almost totally obscured by the squamosal process of the postorbital, which fits into a deep lateral fossa, almost a groove, in the anterior process (Figures 2, 29, 30). More derived iguanodontians (Figure 7, e.g., Mantellisaurus atherfieldensis and Iguanodon bernissartensis) have a greater amount of the anterior process exposed along the intertemporal bar (Character 41:0).

Below the fossa in the anterior process is a sulcus for the origin of $M$. adductor mandibulae externus superficialis (Ostrom, 1961). Below the sulcus is the hooked prequadratic process (Figures $2,30)$. The prequadratic process ends in a point where it meets the squamosal process of the quadratojugal. The process lies along the head of the quadrate, which inserts into a deep fossa underneath (Figures 2, 30). This fossa is roughly hemispheric as in most ornithopods (e.g., Hypsilophodon foxii, Edmontosaurus regalis). Just behind the quadrate is the small, thin postquadratic process, which mimics the shape and orientation of the larger paroccipital process below and lies along its dorsolateral edge (Figure 2). The postquadratic process appears to be the most variable feature of the squamosal among the ornithopods. It is not present in Zalmoxes robustus and supports the head of the quadrate from behind to varying degrees throughout more derived iguanodontians and hadrosaurs (Figure 7) without any obvious phylogenetic pattern.

Orbit. The preserved left orbit of OMNH 58340 has straight dorsal and anterior borders, a gently curving ventral one, and a relatively sharp angle behind (Figure 2). Taken together, this gives a subtriangular outline to the orbital fenestra, rather than subrectangular as originally described and figured (Ostrom, 1970, figure 8). The borders of the orbital fenestra of Tenontosaurus tilletti comprise the lacrimal and prefrontal in front; the prefrontal, frontal, and postorbital above; the postorbital behind; and the jugal below (Figure 2). Generally, the proportions of the contributions of these elements to the fenestra are more similar to those of most basal iguanodontians (Figure 7, e.g., Iguanodon bernissartensis, Ouranosaurus nigeriensis) than to basal ornithopods (e.g., Hypsilophodon foxii). Relative to skull height, however, the overall size of the orbit (Figure 2) compares well with that of $H$. foxii (Galton, 1974a, figure 3).

The orientation of the underside of the frontal, which comprises most of the dorsal border of the orbit (Figure 21, Appendix 4: Frontal), indicates a large eye, although the ocular dimensions are made difficult to determine without preservation of the ethmoidal elements of the skull. Similarly, the outer extent of the eye is unknown due to the lack of an articulated palpebral in OMNH 58340. The angle and position of the optic nerve foramen in the orbitosphenoid indicate that the eye, or at least the entry point of the optic nerve into the eye, was located toward the upper end of the posterior orbit (Figure 2).

\section{Cranial Kinesis}

Analysis of the skull of OMNH 58340 has helped determine the nature of cranial kinesis in Tenontosaurus tilletti. Significant overlap of elements or sutures rule out the possibility of prokinetic, mesokinetic, and metakinetic movements.

As described by Norman (1984) for basal ornithopods and iguanodontians and by Norman and Weishampel (1985) for hadrosaurs, there are two articulated units that would have facilitated pleurokinesis in Tenontosaurus tilletti. The first, toothbearing unit comprises the maxilla, the lacrimal, the palatine, the pterygoid and ectopterygoid, and the jugal and quadratojugal. The second unit comprises the premaxilla, the nasal, the prefrontal and frontal, the postorbital and squamosal, and the parietal, which anchored this unit to the endocranium (see description of the parietal below). A joint 


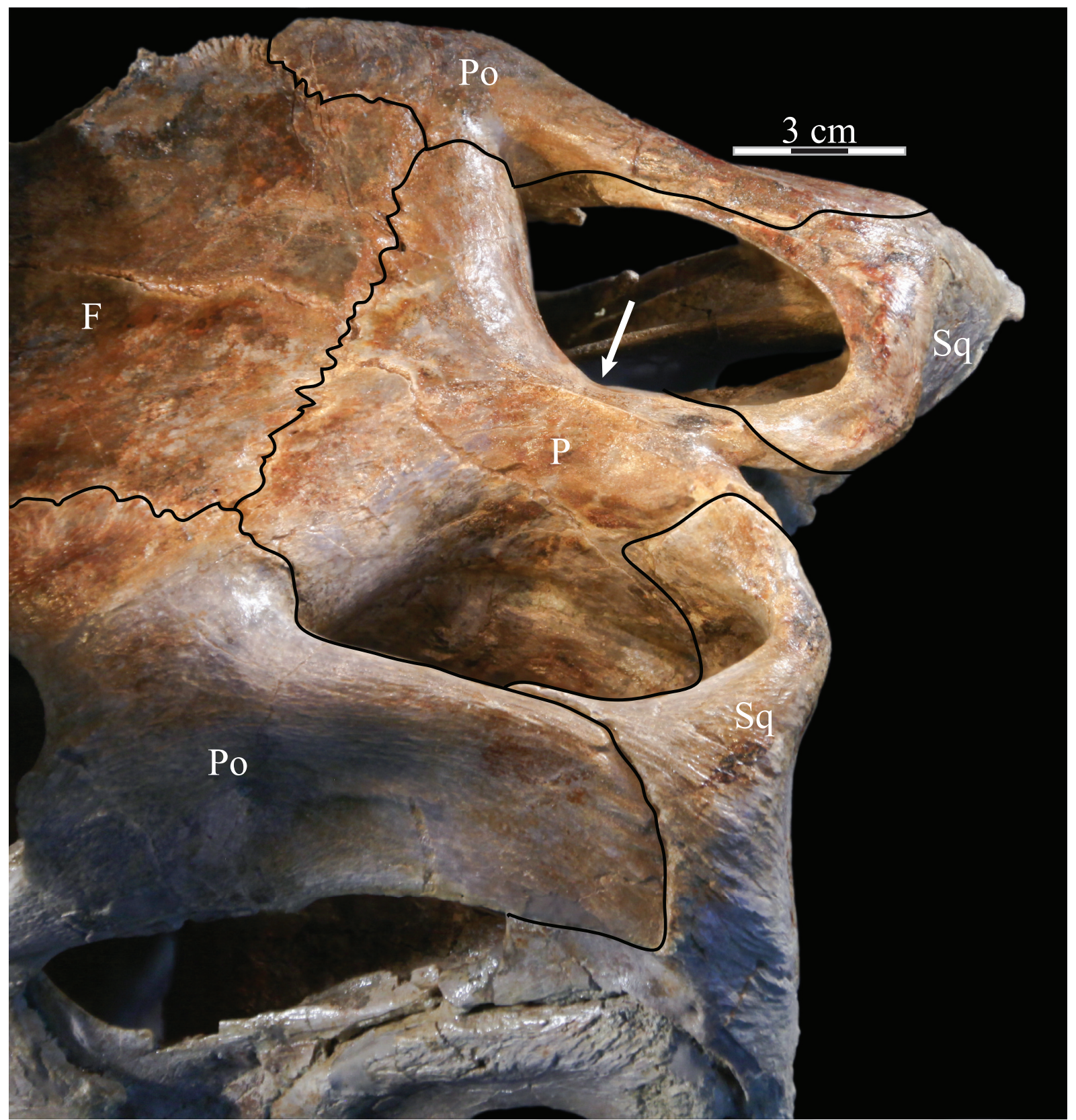

FIGURE 33. Photograph of the posterior portion of the dorsal surface of the skull of OMNH 58340, showing the small waist of the parietal (indicated by arrow). This groove may have been for the passage of an artery or may mark the separation between two muscles. There is an identical feature on the opposite side of the skull. Anterior is to the left.

running between these two units would have allowed the lower part of the first unit to swing out while the second remained stable, corresponding to the upward stroke and inward rotation of the mandible. However, a longer contact between the jugal and postorbital in T. tilletti (Figures 2, 15) probably allowed less movement than was possible in iguanodontians more derived than Dryosaurus altus (Figure 7).
Streptostylic movement between the quadrate and other elements is also likely. The rounded head of the quadrate fits well into the socket formed between the pre- and postquadratic processes of squamosal, and may have allowed transverse and axial movement of the quadratic articular condyles below. It also appears that the jugal, quadratojugal, and quadrate could slide over each other axially and, in the case of the quadratojugal and jugal, dorsoventrally (Figure 2). Finally, the 
pterygoid wing of the quadrate overlaps the quadratic process of the pterygoid, making sliding displacement possible at this joint as well.

Together, these features indicate outward displacement of the lower maxilla, hinging along the lower border of the nasal and premaxilla, together with forward and lateral displacement of the jugal, quadratojugal, and quadrate to compensate. The quadrate could also swing out in order to track with mandible as it pivoted inward during chewing. The vomer, which joins the two sides of the skull, appears to limit lateral displacement of the above elements, in addition to the maxillary adductor musculature proposed by Norman (1984). However, elastic deformation of the long, thin posterior processes, which attach solidly to the upper pterygoid, would have provided mechanical impetus to return to an unstressed resting position without expending muscular energy. The vomer is uniformly tall and bending across the vertical axis is unlikely, which corresponds well to a lack of the first three types of kinesis mentioned above. Overall, cranial kinesis in Tenontosaurus tilletti appears to be very similar to, if not as progressed as, that described for other basal iguanodontians (Norman, 1984) and hadrosaurs (Weishampel, 1983).

\section{Neurocranial Series}

In OMNH 58340, the braincase is preserved in articulation with the rest of the cranium, with little or no apparent distortion. There are a few imperfections in preservation, likely due to over-preparation, which will be noted as they arise.

Parietal. The parietal is tall and completely fused across the midline of the skull, as in all mature ornithopods. It is T-shaped in dorsal aspect, with paired lateral processes forming the bars of the " $T$ " and smaller occipital processes extending behind (Figures 1, 5, 9, Character 45:1, Character 46:0). The parietal of Tenontosaurus tilletti most closely resembles that of $T$. dossi.

The parietal forms a flat, strongly interdigitate border with the frontals anteriorly (Figures 5, 34) and with the postorbitals laterally (Character 17:0). The processes and the body of the parietal form the anterior and medial borders of the superior temporal fenestrae, respectively (Figure 5).

Beginning halfway between the midline of the skull and the outer edge of each lateral process is a pair of weak crests, which become stronger posteromedially and join to form a weak sagittal crest, a feature common to all ornithopods (Figure 5). The crest becomes a shallow furrow just before the bone splits into the occipital processes (Figure 33,
Character 47:0). A pair of shallow grooves runs forward on either side of the crest and forms a waist about the middle of the bone (Figure 33). This is the only feature in the area that could correspond to the separation of the $M$. adductor mandibulae externus profundus and $M$. pseudotemporalis suggested by Ostrom (1961), although he reported a crest separating the probable sites, not a groove.

The parietal divides into two flaring, wing-like occipital processes behind, which are overlapped by the medial processes of the squamosals and which terminate just above the squamosal bosses of the supraoccipital (Figures 1,33). The occipital processes of the parietal form a hood over and around the ascending process of the supraoccipital. The parietal and supraoccipital are highly fused with no readily discernible suture, although the latter element appears to end near the dorsal sagittal venous sinus of the endocast (see description of the endocast below).

The bone is strongly concave for the braincase below (Figure 21) and narrows into a waist that then flares into the overlapping articulation with the supraoccipital behind (Figure 1). The endocranial cavity itself ends abruptly after becoming roofed by the supraoccipital. The braincase quickly narrows under the parietal from its greatest width under the frontals (Figure 21).

On either side of the endocast are the sturdy pedicles of the parietal that articulate with the supraoccipital behind and with the flat heads of the laterosphenoids in front (Figures 34, 35). The parietal does not contact the prootic below (Character 49:1), as it does in all ornithopods more derived than Tenontosaurus tilletti (Figure 7, e.g., Iguanodon bernissartensis, Corythosaurus casuarius). As in other taxa basal to T. tilletti (Figure 7, e.g., Hypsilophodon foxii, T. dossi), the parietal is excluded from this contact by the anterior process of the supraoccipital.

Opisthotic and Exoccipital. Although the opisthotic and exoccipital likely make distinct contributions to the lateral and posterior braincase, as well as to the paroccipital processes, they are completely fused and indistinguishable in OMNH 58340 and in the smaller (and ontogenetically younger) OMNH 2531. The front of the fused element, which makes up the rear of the lateral braincase, is presumed to be the opisthotic while the exoccipital behind presumably comprises most of the paroccipital processes and part of the borders of the foramen magnum (Figures 1, 35). They are referred to as such. 


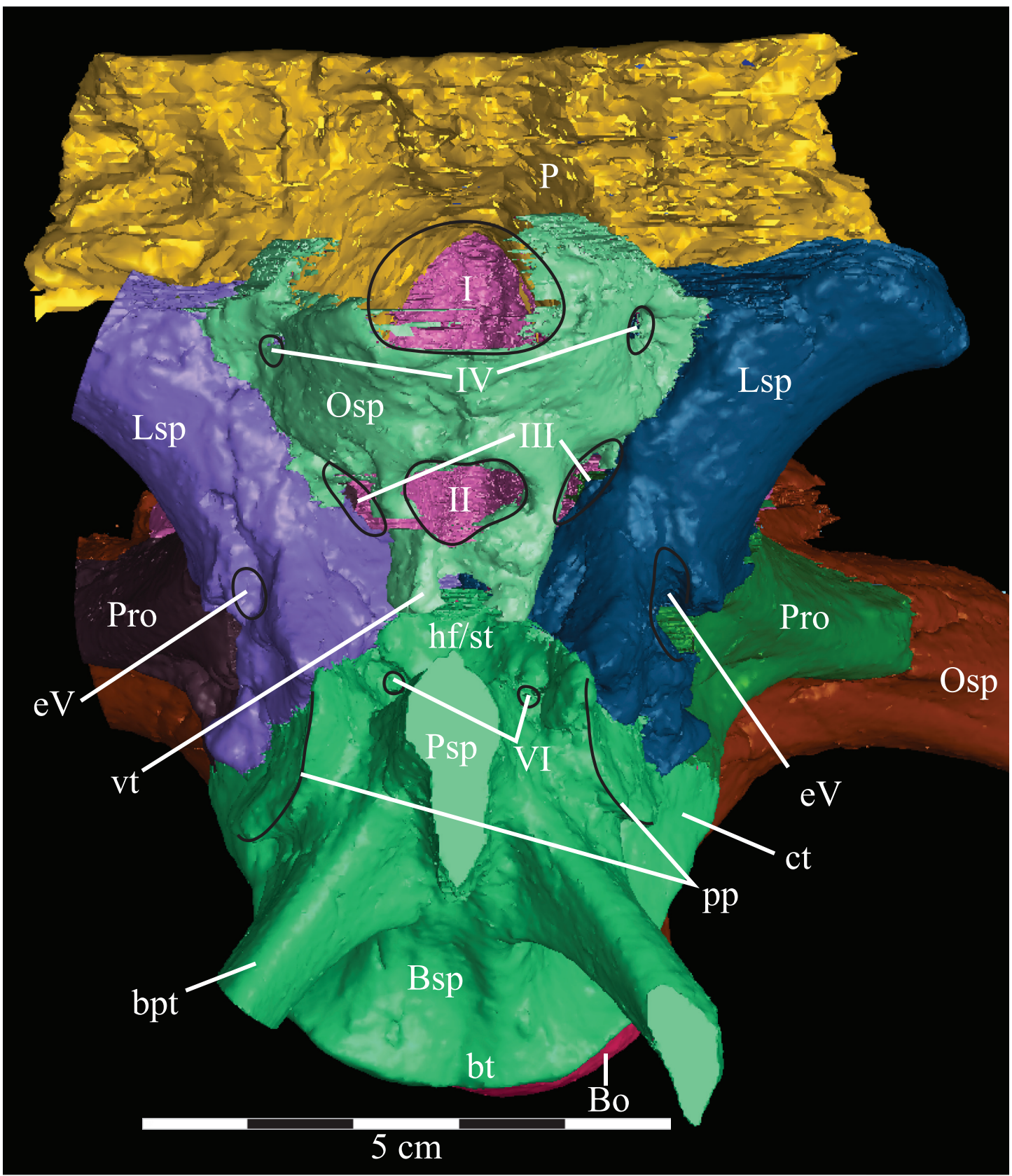

FIGURE 34. Anterior view of the virtual braincase of OMNH 58340. The supraoccipital is indicated by the pink that shows through the foramina for CN I-III. The smooth area across the front of the parasphenoid and the left basipterygoid process, as well as the regular curvature of the outer edge of the right laterosphenoid, prootic, and opisthotic (to the left), are artifacts of CT scanning and represent the limits of the data set. Note the irregular anterior surface of the parietal, showing the interdigitate nature of the suture of that element with the frontals. Black lines are used to denote particular features. Anterior is out of the page and ventral is down. Abbreviations: bt - basal tubera; bpt - basipterygoid process; ct - crista terminalis; eV - excavation for trigeminal nerve (CN V); hf - hypophysial fossa; pp - preotic pendant; st - sella turcica; vt - ventral tubercle of the orbitosphenoid.

The opposite sides of the element are joined by a small bar, triangular in sagittal section (Figures 1,36$)$, which creates the dorsal edge of the foramen magnum and excludes the supraoccipital from participation in its border (Character 43:1).
The posterior face of the exoccipital has the same texture as the adjoining area of the supraoccipital (Figure 1, see description of the supraoccipital below). 


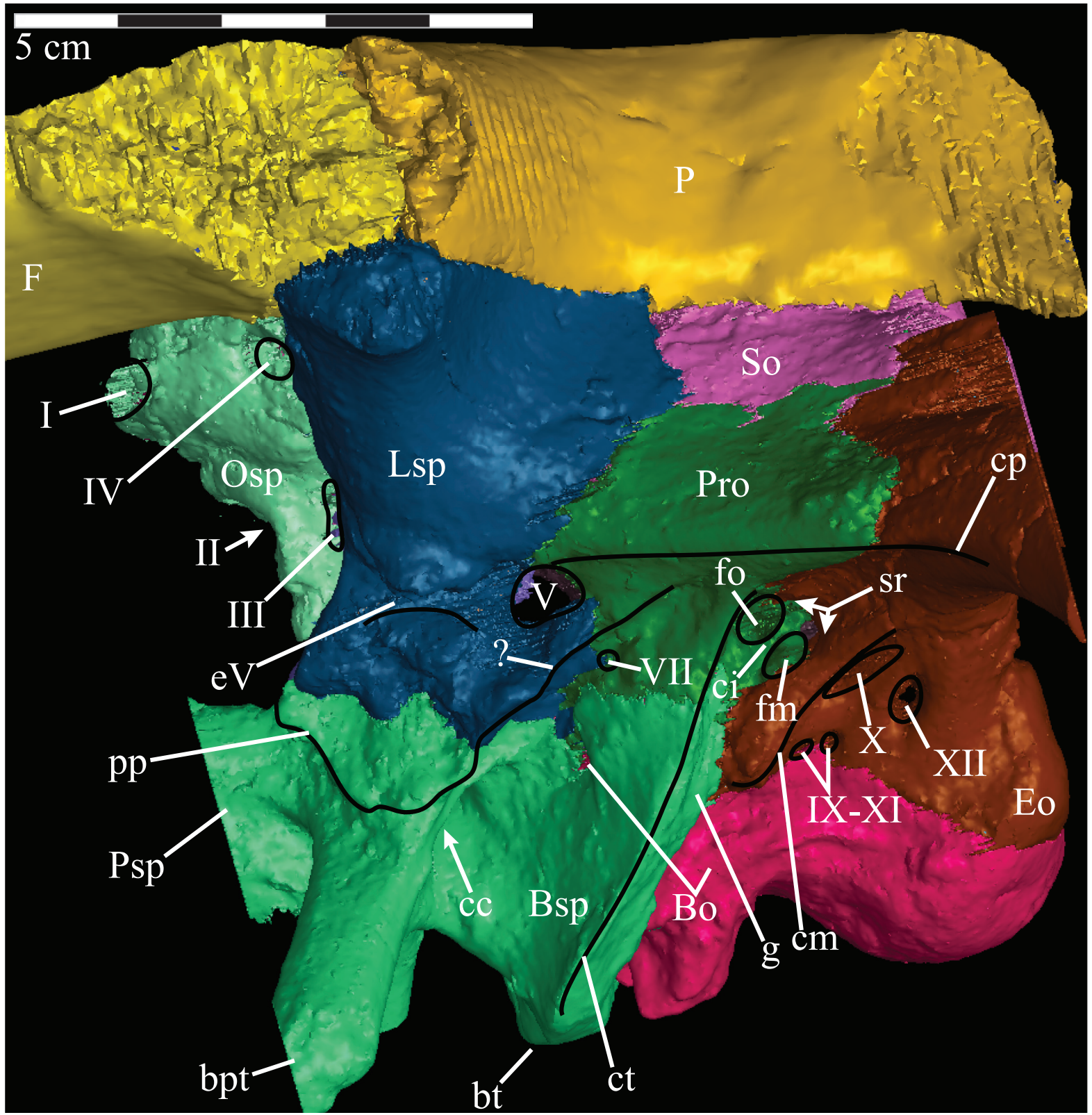

FIGURE 35. Lateral view of the virtual braincase of OMNH 58340. Black lines are used to denote particular features. The straight lines at the front of the basisphenoid to the left and the exoccipital/opisthotic to the right are artifacts of the CT scanning and represent the limits of the data set. The frontal and parietal extend beyond these levels because they were transplanted from the full cranial scan. Note that the basioccipital is visible in two locations. Anterior is to the left. Abbreviations: bt - basal tubera; bpt - basipterygoid process; cc - internal carotid canal (obscured); ci - crista interfenestralis; $\mathrm{cm}$ - crista metotica; $\mathrm{cp}$ - crista prootica; ct - crista tuberalis; eV - excavation for trigeminal nerve (Cranial Nerve V); fm - fenestra metotica; fo - fenestra ovalis; $g$ - groove of crista tuberalis; pp - preotic pendant; sr - stapedial recess; ? - unnamed ridge.

Above the foramen magnum, the exoccipital sends up a pair of dorsally tapering processes around the squamosal bosses of the supraoccipital (Figure 1). Between these processes, above the foramen magnum, the exoccipital flatly abuts the supraoccipital. The exact margins of the two ele- ments are almost impossible to distinguish here, due to a high level of fusion. A pair of bosses just below the transverse bar cause undulation in the dorsal edge of the foramen magnum (Figure 1). There are two shallow fossae next to these bosses, similar to the ones described for Theiophytalia kerri 


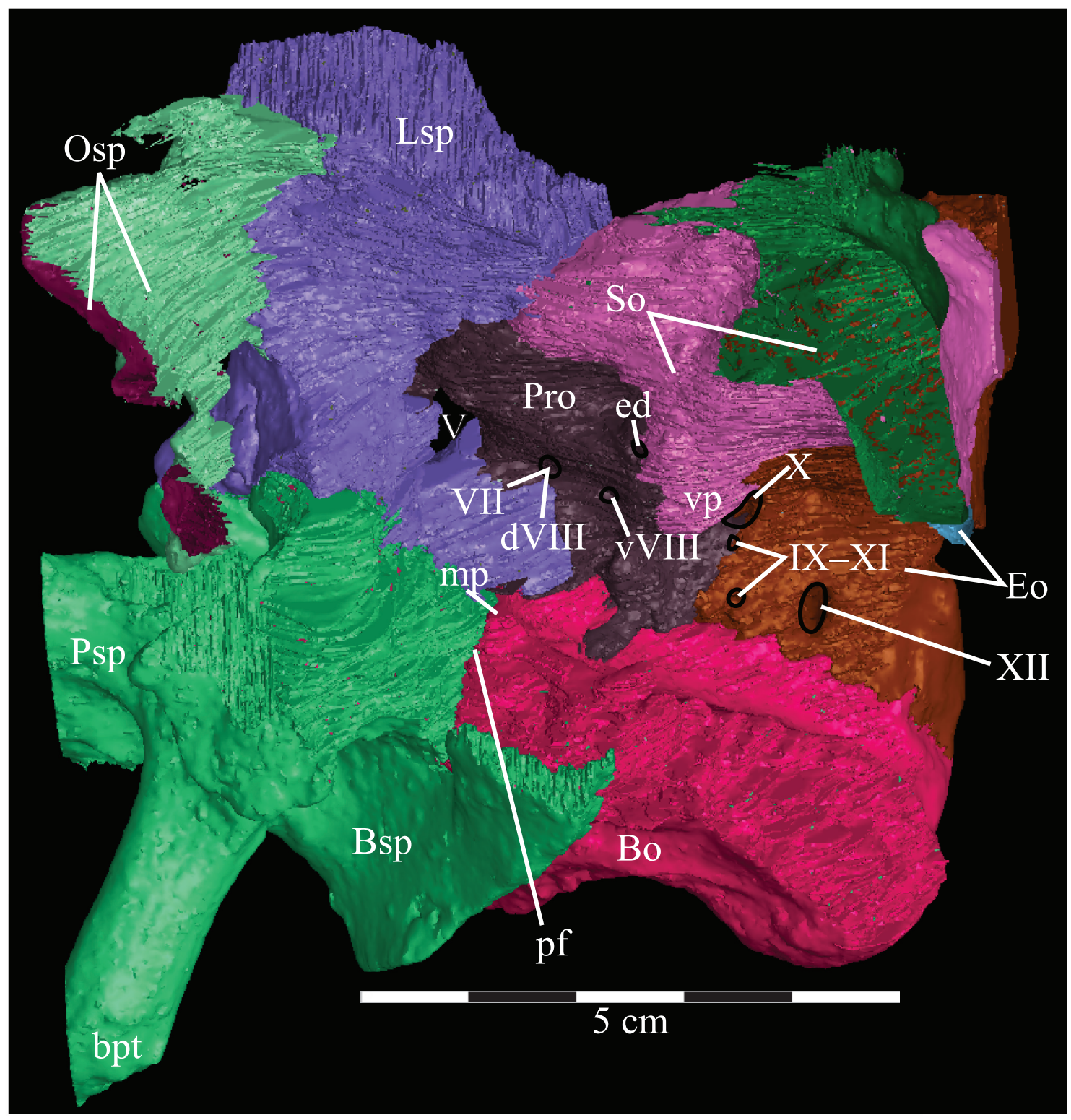

FIGURE 36. Dorsolateral view of the virtual braincase of OMNH 58340 with the parietal, left laterosphenoid, left prootic, and left sections of the supraoccipital and exoccipital/opisthotic removed. The flat edges of the parasphenoid and of the supraoccipital and exoccipital to the left and right, respectively, are artifacts of the CT scanning, and represent the limits of the data set. The supraoccipital (pink), exoccipital (brown), and orbitosphenoid (light green) were sectioned to allow for viewing of structures that would normally be covered by these elements. The computer program used to do this randomly assigns interior colors to the models, hunter green in the case of the supraoccipital, light blue in the case of the exoccipital, and maroon for the orbitosphenoid. Anterior is to the left. Abbreviations: bpt basipterygoid process; dVIII - dorsal ramus of the acoustic nerve; ed - endolymphatic duct; mp - median process of the basioccipital; pf - area of the pontine flexure; vp - ventral process of the supraoccipital; vVIII - ventral ramus of the acoustic nerve.

(Gilmore, 1909, figure 4), although perhaps not as pronounced.

The exoccipital extends below on either side of the foramen magnum, expanding into condylids (Weishampel and Bjork, 1989) that exclude the basioccipital from all but about a quarter of the foramen (Figure 1). The condylids form the upper third of the bulbous occipital condyle. The articular surface with the basioccipital below is broadly interdigitate and preserved unfused. A similar condition was noted for Hypsilophodon foxii (Galton, 1974a). 
As the exoccipital extends outward into the paroccipital processes (Figure 1), its dorsal edge rotates back and the ventral edge forward until it terminates in a point (Figures 1, 2,6). The superficial tympanic membrane was likely located here, between the paroccipital process and the neck of the quadrate (Figure 2, see description of the quadrate above). The tip of the paroccipital process also likely served as the origin of $M$. depressor mandibulae. The processes do not form a dramatic angle in dorsal aspect, as figured by Ostrom (1970, figure 9), but instead are nearly straight (Figures 5, 6).

Norman (1980) outlined the evidence for the composition of the paroccipital process in Ornithopoda. Galton (1974a) described the opisthotic of Hypsilophodon foxii as contributing primarily to the process, while Langston (1960) demonstrated that the exoccipital of hadrosaurs was the dominant element. Norman (1980) suggested an intermediate state for Iguanodon bernissartensis. This is likely also the case in Tenontosaurus tilletti.

The front of the paroccipital process is marked by a sharply convex ridge extending from the crista prootica of the prootic, which overlaps the opisthotic in a pointed posterior process (Figure 35). The stapedial recess lies just forward of the opisthotic, below the crista prootica (Figure 35). The opisthotic does not directly contribute to the border of the fenestra ovalis.

Below the stapedial recess is a thin ridge, the crista metotica, which continues forward from the base of the paroccipital process to interject between the basioccipital and basisphenoid, just behind the crista tuberalis of the basisphenoid (Figure 35). The anterior end of the crista is the farthest anterior point of the opisthotic.

The foramen for the vagus nerve $(\mathrm{CN} \mathrm{X})$, which opens below the crista metotica, is fairly large, about the same size as the fenestra ovalis above (Figure 35). Directly below this foramen are two smaller, subequal foramina, which are here tentatively assigned to the glossopharyngeal nerve (CN IX) in front and the spinal accessory nerve ( $\mathrm{CN} \mathrm{XI)} \mathrm{behind.} \mathrm{Alternatively,} \mathrm{the} \mathrm{glossopharyngeal}$ nerve may, with the jugular vein, have exited through the fenestra metotica (Figure 35) of the auditory foramen above, and this small foramen may have carried other cerebral vasculature. The hypoglossal canal (CN XII), which is roughly the same size as the vagus nerve foramen, opens behind all three of these foramina. The opisthotic/ exoccipital entirely contain all four of these foramina, although the foramina for IX and XI open very near the basioccipital below (Figure 35). Weishampel et al. (2003) described a similar spatial arrangement for the cranial nerve foramina in $\mathrm{Zal}-$ moxes robustus, although the landmark those authors used was the crista tuberalis, not the ridge below the stapedial recess described here for Tenontosaurus tilletti.

Medially, the ventral tip of the lagena of the cochlea is in the opisthotic, adjacent to the crista metotica. The posterior half of the lateral and the ventral half of the posterior semicircular canals pass through the opisthotic (see description of the endosseous labyrinths below). Neither the anterior canal nor the common crus pass through this element, although the common crus may make a slight contact at its base. The rear of the flocculus may make contact with the element, but this is doubtful.

The paired halves of the element around the cranial nerve foramina are directed medially, helping form a waist for the medulla of the endocranial cavity (Figure 36). The opisthotic slopes inward, but is replaced by the basioccipital before it can form any portion of the floor of the braincase.

Supraoccipital. The supraoccipital appears roughly triangular behind, with paired squamosal bosses (Langston, 1960) below and an ascending median process above (Figures 1, 37). This configuration most closely resembles the inverted " $T$ " of Tenontosaurus dossi in general appearance. A pair of ridges and grooves runs between the bosses and the process, flanking the base of the latter (Figure 37).

The ascending process has a flat top that projects behind, forming a nuchal shelf (Figures 1, 37). The median tip of the process continues below as a ridge, which divides and then recedes into the flat surface beneath. This flat surface has a texture unique to this area of the skull, which is present here and immediately below on the exoccipital (see description of the opisthotic and exoccipital above), just above the foramen magnum. It appears corrugated, with the tops of the transverse ridges shaved away, leaving very shallow, sharply edged furrows (Figure 1). This area probably served as the point of insertion for M. spinalis capitis (Ostrom, 1961). Above the squamosal bosses, as in Tenontosaurus dossi, are the two posterior temporal foramina, which are bordered above by the squamosals and likely provided the passage of the vena capitis dorsalis.

The supraoccipital is excluded from participation in the foramen magnum by a thin transverse bar of the exoccipital (Figures 1, 36, Character 


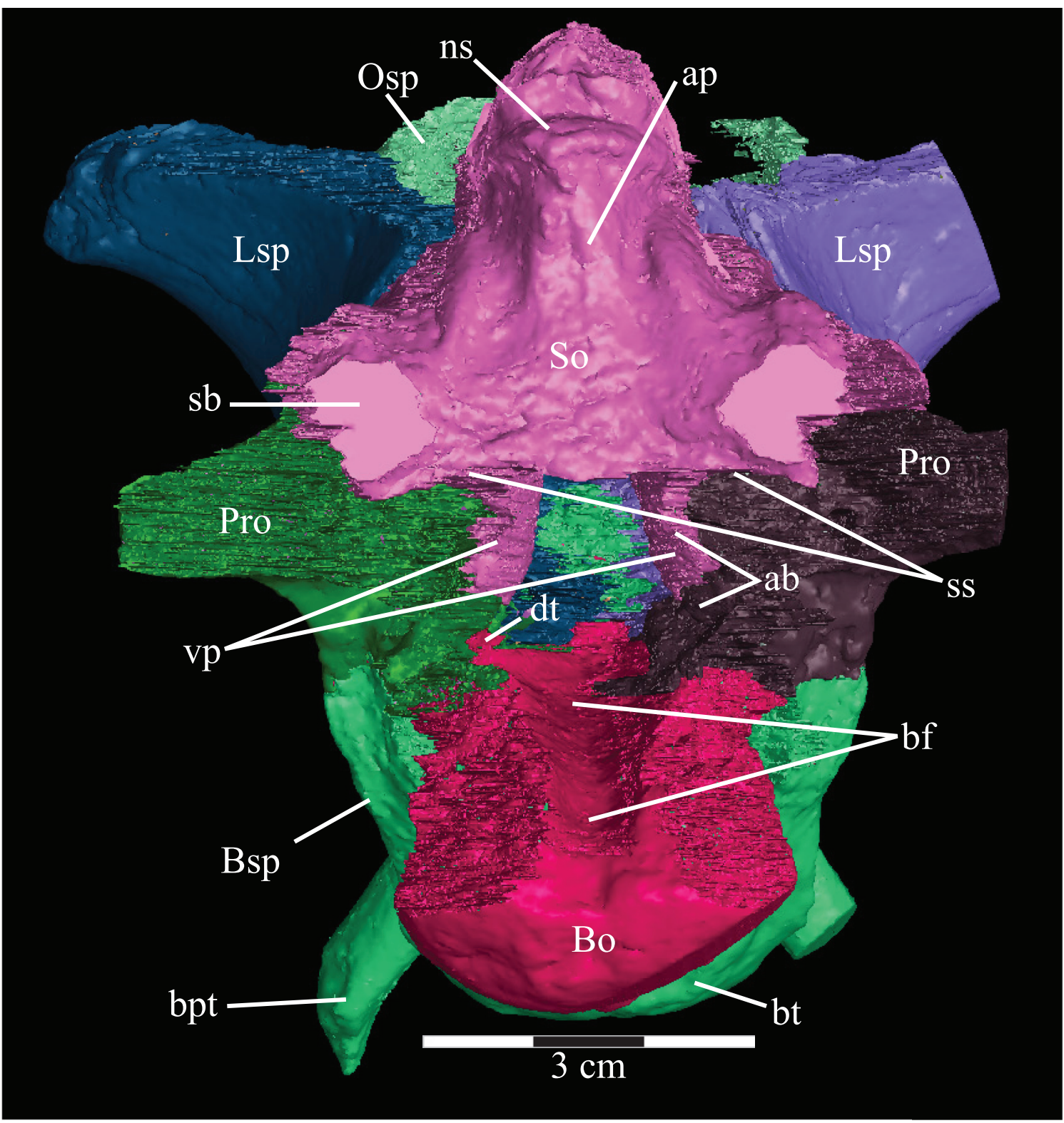

FIGURE 37. Posterior view of the virtual braincase of OMNH 58340 with the exoccipital/opisthotic removed. The regularly curved borders along the right side, as well as the flat squamosal bosses of the supraoccipital, are an artifact of the CT scanning and represent the limits of the data set. Anterior is into the page and dorsal is up. Abbreviations: ab - auditory bulla; ap - ascending process; bf - basioccipital furrow; bt - basal tubera; bpt - basipterygoid process; dt dorsal tubercle; ns - nuchal shelf; sb - squamosal boss; ss - supraoccipital shelf; vp - ventral processes.

43:1). This condition is shared with Thescelosaurus neglectus, Tenontosaurus dossi, and with all ornithopods more derived than Mantellisaurus atherfieldensis (Figure 7; Norman, 1990).

The supraoccipital continues briefly above its nuchal shelf and actually flares posteriorly again to roof over the shelf (Figure 37). The fossa in between, which is continued by the parietal and squamosals (Figure 1), probably served as the point of attachment of the nuchal ligament (Ostrom, 1961). The supraoccipital continues forward under the parietal to roof the posterior endocranial cavity. The parietal excludes the supraoccipital from articulation with the squamosals.

The dorsal extent of the supraoccipital is difficult to discern, especially forward, due to fusion with the overlying parietal. The supraoccipital 
appears, however, to form a dorsal arch, below which the element forms a shelf, abutted above by the parietal, which is continuous with the top of the squamosal bosses (Figures 37, 38, 39). The supraoccipital has two partially exposed anterior processes (Figures 35, 38), which slope downward before they are sharply undercut to become shelves (Figure 38, Appendix 4: Supraoccipital). These processes abut the exoccipital and prootic below and the parietal above (Figures 35, 39). The ventral surface of the supraoccipital primarily comprises a flat, almost entirely fused, suture with the exoccipital below. The posterior processes of the laterosphenoids (see description of the laterosphenoid below) overlap the tips of the anterior processes (Figure 39). Such a forward extent of the supraoccipital is, apparently, a basal character shared among Tenontosaurus tilletti and basal ornithopods (e.g., Hypsilophodon foxii). The supraoccipitals of ornithopods more derived than T. tilletti appear to be restricted behind; that is, they do not participate externally in the lateral braincase. However, based upon observations in OMNH 58340 (Figures 5, 35, 39), including the high degree of fusion between the supraoccipital and its neighbors, it is possible that the supraoccipital is similarly present in the derived taxa with indeterminate borders.

The only other significant feature on the underside of the supraoccipital is a pair of ventral processes that extend down from the element (Figure 37). These processes appear lighter in the CT scan, indicating a higher density (Figure 40), and, in ventral aspect, are laterally concave and angled slightly forward (Figure 36, Appendix 4: Supraoccipital). Together with the prootics, these processes form medial auditory bullae and help house the endosseous labyrinths (Figure 38), containing the upper halves of the anterior and posterior semicircular canals as they join to form the common crura. Another feature, the endolymphatic duct canal (cf. Witmer and Ridgely, 2009), is shared between the ventral process of the supraoccipital and the prootics (Figure 36). The ventral processes contact the exoccipitals/opisthotics behind, the prootics in front, and both laterally (Figure 36).

In anterior aspect, the most prominent feature of the supraoccipital is a deep groove, the dorsal sagittal sinus, running vertically along the midline (Figure 41). This furrow is widest below, thins in a waist at its middle, and then flares again dorsally. The cerebellum was likely housed in two medially oriented concavities in front of the waist (Figure 41). Between these concavities and the ventral processes are the conical (subarcuate) fossae that contained the flocculi (Figure 41), which are completed by the prootics below. The borders of the fossae are similarly shared in basal ornithopods (Figure 7). The supraoccipital contributes a significant portion to the walls of the braincase, laterally, dorsally, and, most significantly, posteriorly (Figure 41).

Basioccipital. The basioccipital is a median element, triangular in lateral aspect with the posterior and anterior ends separated below by a deep groove (Figure 35). A small foramen perforates the basioccipital near the midline in this groove, which traces up into the bone and ends blindly before it reaches the endocranium. The larger rear section of the basioccipital is bulbous and forms the lower two-thirds of the subspherical occipital condyle (Figures 1, 35, Character 42:0). The exoccipital condylids contribute above, as in all iguanodontians and hadrosaurs (e.g., Tenontosaurus dossi, Mantellisaurus atherfieldensis, Corythosaurus casuarius). The bone is quite dense throughout, with closely packed trabeculae.

The anterior section of the basioccipital has two definite points below, a median and a right lateral tubercle, with a poorly preserved left lateral tubercle (Figure 6). The lateral tubercles are offset just forward of the median tubercle, which appears triangular from below. The right lateral tubercle is continuous with the basal tuber of the basisphenoid. The basioccipital does not contribute to the formation of the basal tubera, as it does in some basal iguanodontians (e.g., Ouranosaurus nigeriensis) and hadrosaurs.

There is a median furrow that comprises most of the floor of the endocast running forward over the bone (Figures 37, 42). The furrow is roughly diamond-shaped, with two small processes at its widest point (Figure 37) and a small median process at its anterior tip (Figures 39, 42). The latter process corresponds roughly to the pontine flexure of the endocast.

The anterior face of the basioccipital, which abuts the basisphenoid, is flat (Appendix 4: Basioccipital). The element is bounded laterally, from its front to the level of the ventral groove, by the crista tuberalis of the basisphenoid (Figure 35). A small corner of the basioccipital is visible externally between the body of the basisphenoid and its crista, although this may be due to incomplete preservation (Figure 35). The crista metotica of the opisthotic replaces the basisphenoid as border behind the crista tuberalis. The opisthotic forms an abutting suture with the outside of the basioccipital. 


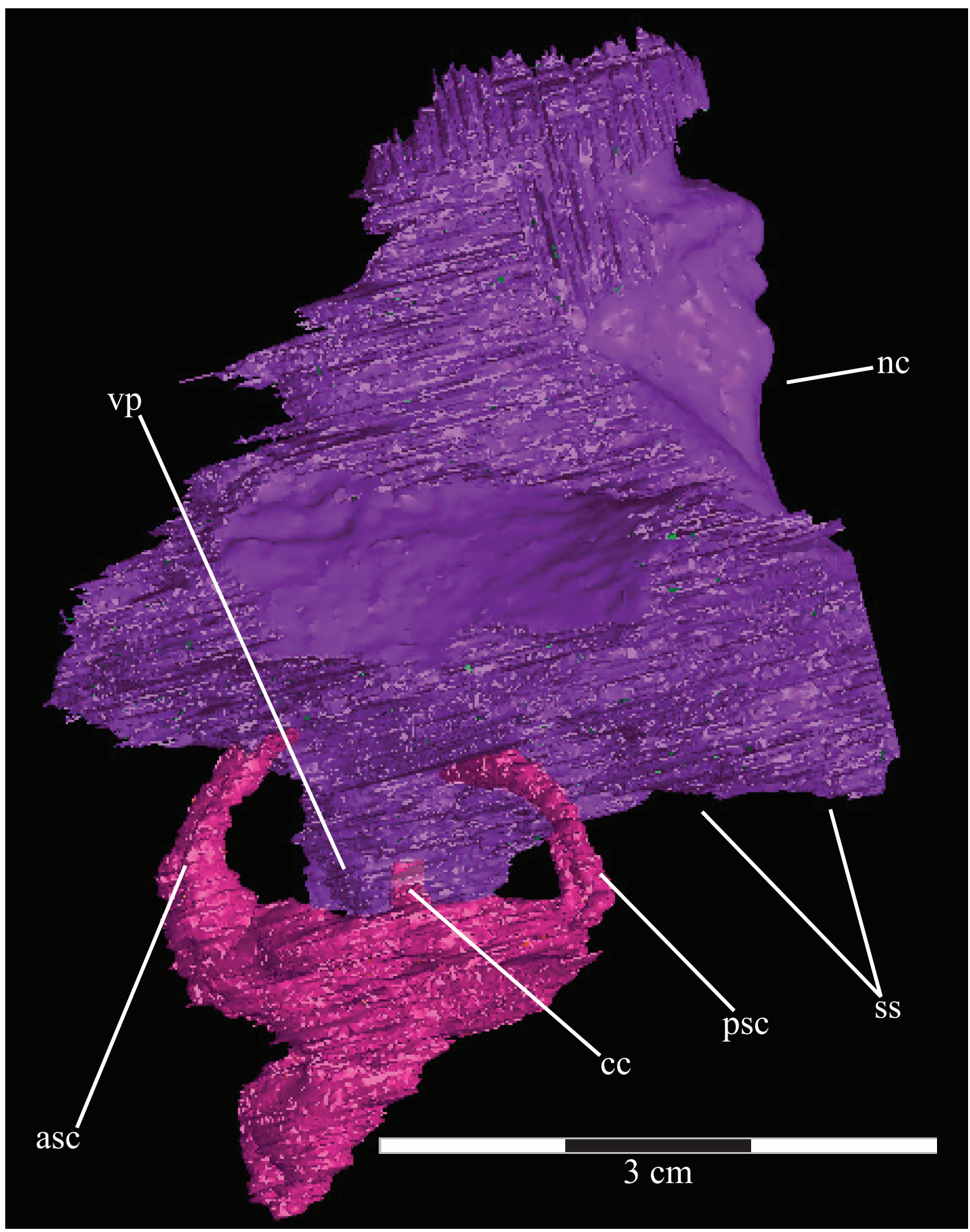

FIGURE 38. Lateral view of the virtual supraoccipital (purple) and the left endosseous labyrinth (pink) of OMNH 58340 showing the common crus of the semicircular canals entering the ventral process of the supraoccipital. Anterior is to the left. Abbreviations: asc - anterior semicircular canal; cc - common crus; nc - nuchal crest; psc - posterior semicircular canal; ss - supraoccipital shelf; vp - ventral process. 


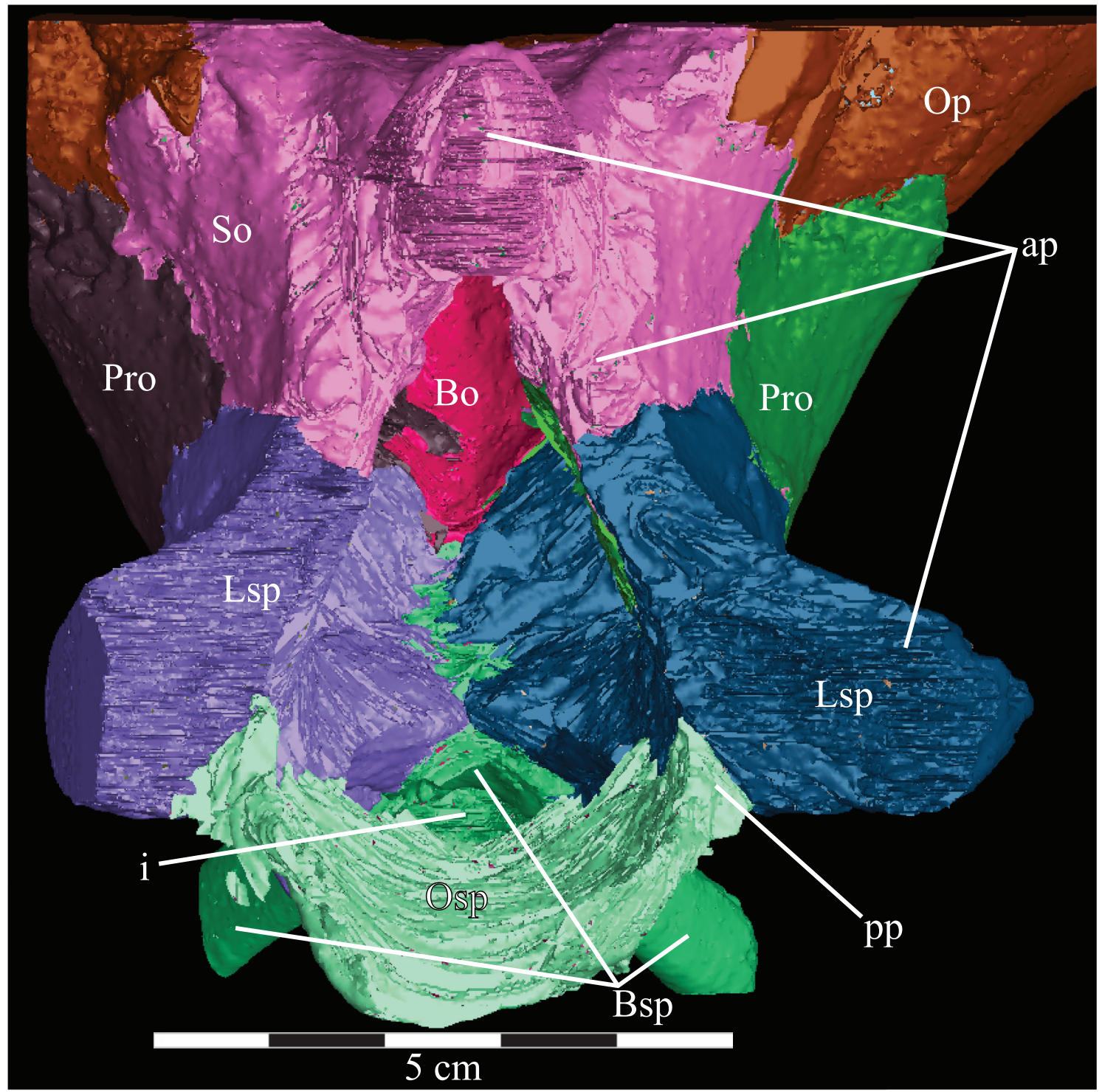

FIGURE 39. Dorsal view of the virtual braincase of OMNH 58340 with the parietal removed, showing the composition of the floor of the braincase. The straight posterior edge of the opisthotic, as well as the straight anterior edge of the basisphenoid and lateral edge of the laterosphenoid, are artifacts of the CT scanning and represent the limits of the data set. Anterior is down. Abbreviations: ap - area of articulation with parietal; i - infundibular canal; pp - posterior process of the orbitosphenoid.

The opisthotic/exoccipital then shifts above at the level of the small, paired dorsal processes of the basioccipital to form a strongly interdigitate suture (Figures $35,36,42$ ). The paired processes of the basioccipital are covered by the prootics (Figure 36). The laterosphenoids form the rest of the dorsal border forward of the prootics. Contact with the laterosphenoids has not been described in other related taxa and may represent an autapomorphy for Tenontosaurus tilletti, although it is also likely that this lack of description is due to a lack of avail- ability of specimens of a similar quality and access to CT data.

Basisphenoid/Parasphenoid. The basisphenoid and parasphenoid form another pair of elements that are described as a single unit due to their high degree of fusion and the uncertainty of their boundaries. The front of the elements, protruding between the paired posterior processes of the vomer (Figures 6, 9), will be described as parasphenoid. The rest, because of uncertainty, will be called basisphenoid. There is an apparent internal 


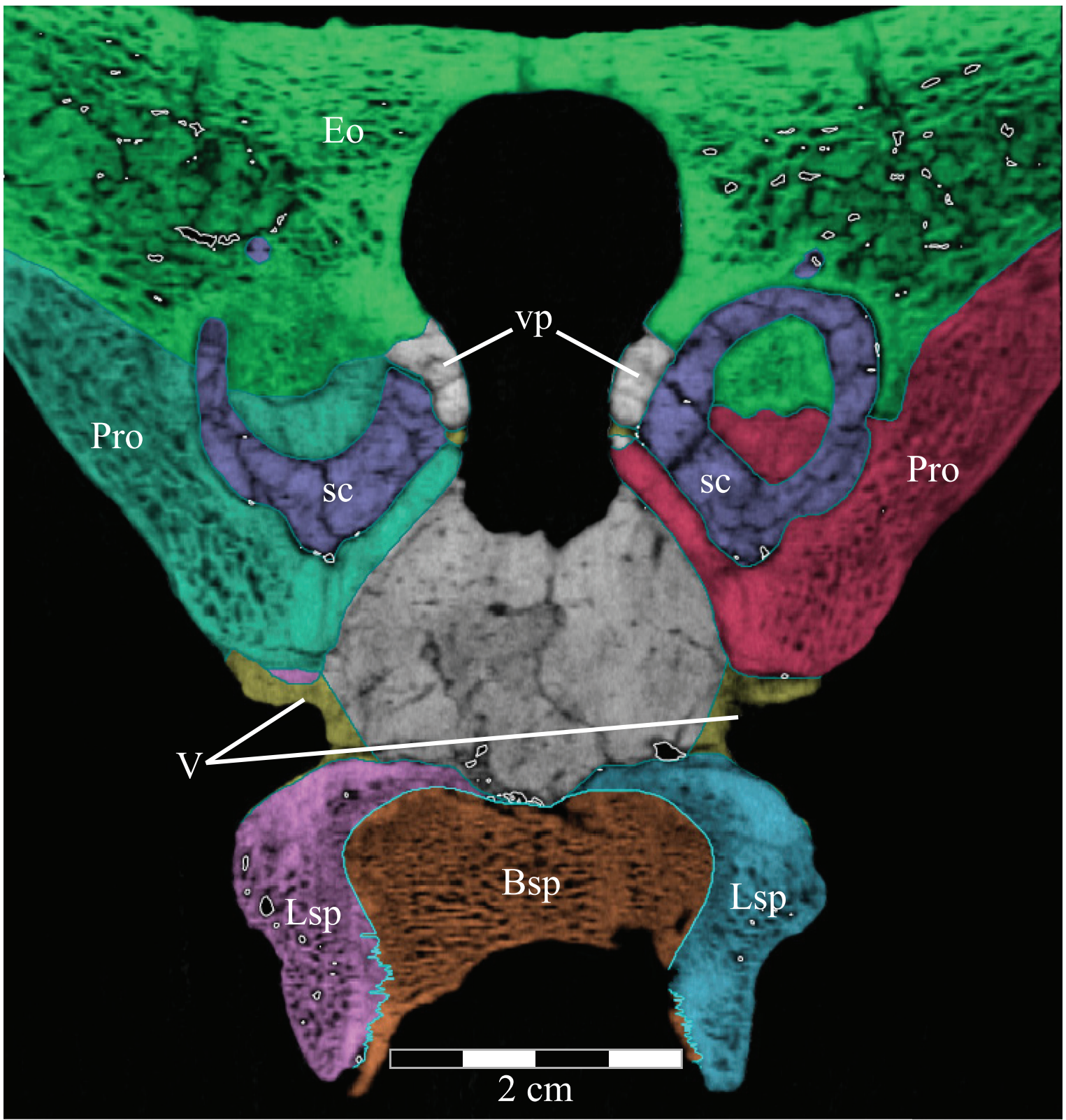

FIGURE 40. Horizontal CT image, taken at the level of the trigeminal foramina, of the basisphenoid and parasphenoid (orange), laterosphenoids (pink and light blue), trigeminal nerve foramina (yellow), prootics (teal and red), horizontal semicircular canals (purple), and exoccipital/opisthotic (green) of OMNH 58340. The endocast is the black and gray area in the middle of the image. The ventral processes of the supraoccipital can also be seen, as well as their peculiar density. Faint green lines are an artifact of the program used to define the element on each image. Anterior is down. Abbreviations: sc - horizontal semicircular canals; vp - ventral processes.

suture near the probable junction of the elements, but it is inconsistent and incomplete. I have noted where possible sutures indicate that the combined elements may be better delineated.

The parasphenoid is long, narrow, and tall (Figure 9, schematic, Character 44:0). In coronal cross-section, the parasphenoid is V-shaped, with a dorsal furrow running along its length (Figure 43). The element extends forward past the orbit and ends in a point between the paired posterior processes of the vomer. The parasphenoid is weakly sinusoidal along its length (Figure 9): convex dorsally behind, then concave dorsally about halfway forward.

The median groove of the parasphenoid may be filled behind with the paired cristae trabeculares of the basisphenoid, which appear to extend into the groove from below and continue about a third 


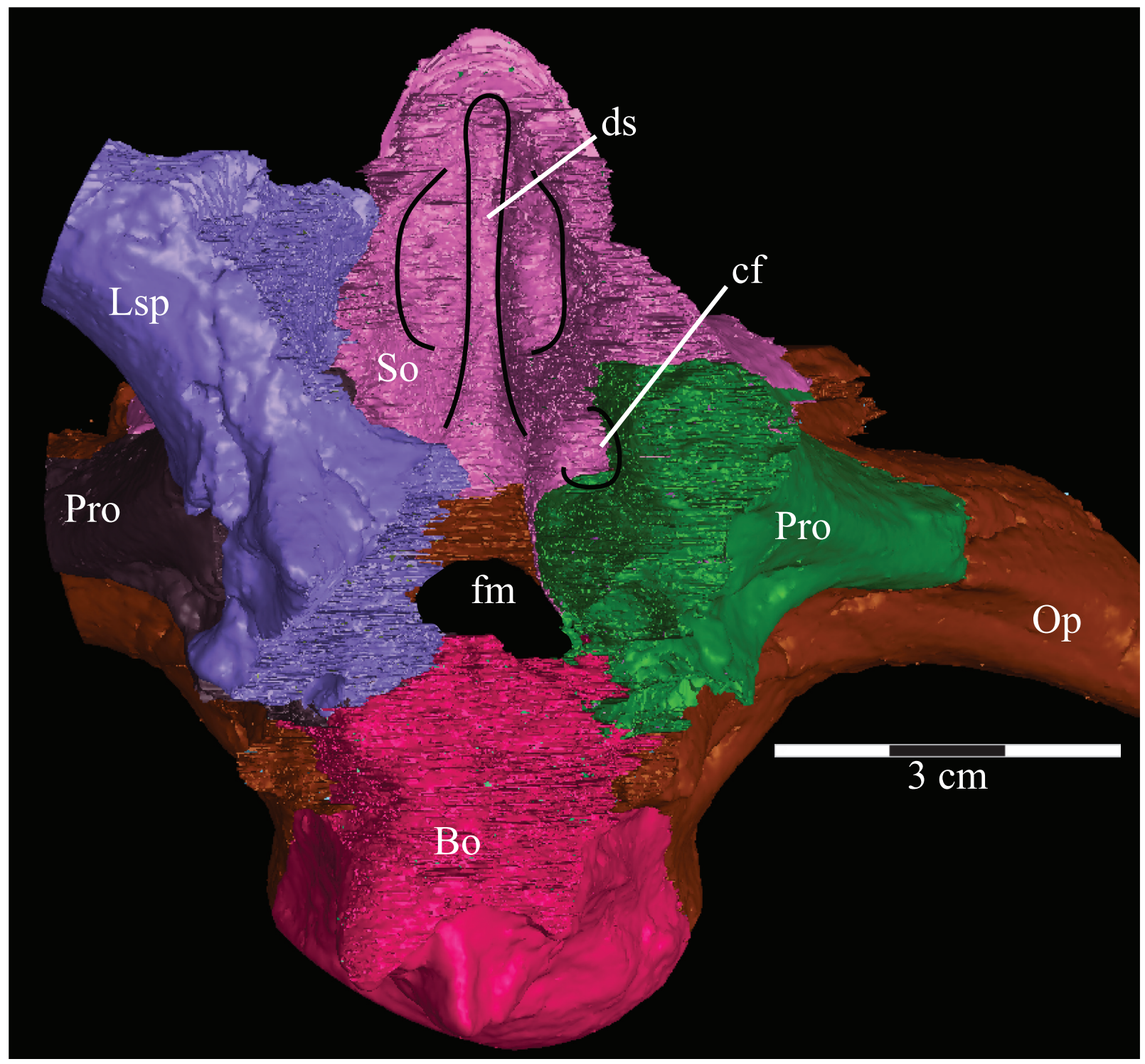

FIGURE 41. Anterior view of the virtual braincase of OMNH 58340 with the parietal, orbitosphenoid, left laterosphenoid, and basisphenoid/parasphenoid removed. The curvature along the left border is an artifact of the CT scanning and represents the limit of the data set. Black lines are used to denote particular features. Anterior is out of the page and ventral is down. Abbreviations: conical fossa; ds - dorsal sagittal sinus; fm - foramen magnum.

of the way forward (Figure 43). Alternatively, this could be a remnant of the presphenoid anteriorly. The groove probably accommodated the paired median palatine arteries, branching from the internal carotid arteries behind (Ostrom, 1961).

In the CT data, the parasphenoid appears to insert between the basipterygoid processes of the basisphenoid behind, and to continue back until nearly reaching the basal tubera; but here, again, its extent is difficult to discern. In Mantellisaurus atherfieldensis and, tentatively, in lguanodon bernissartensis, Norman (1986; 1980) suggested that the parasphenoid continues far enough back to contribute to, or indeed to enclose the basipterygoid processes of the basisphenoid. It is possible that the parasphenoid contributes similarly in Tenontosaurus tilletti.

The basisphenoid is a complex element made of paired basipterygoid processes, paired cristae tuberales continuing down from the prootic, and paired basal tubera underneath (Figures 34, 35). The deep hypophyseal fossa, which probably separates the parasphenoid and basisphenoid, terminates below in two small internal carotid artery foramina (Figures 34, 35). These foramina are quite round and nearly meet on the midline just 


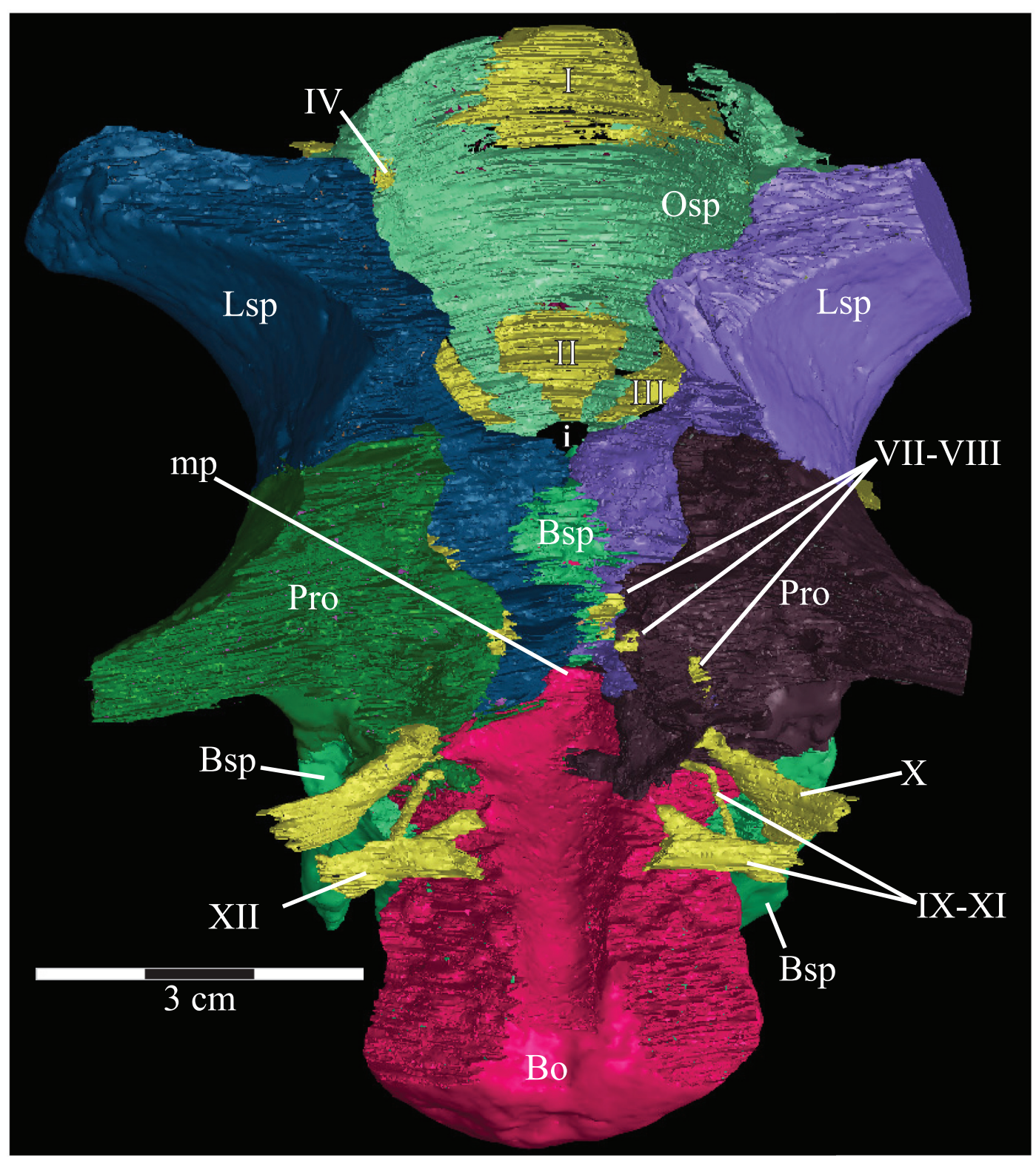

FIGURE 42. Posterodorsal view of the virtual braincase of OMNH 58340 with the parietal, supraoccipital, and exoccipital/opisthotic removed and the paths of the cranial nerves highlighted. Anterior is into the page. Abbreviations: $\mathrm{i}$ infundibulum; mp - median process.

below the fossa. The foramina were alternatively called Vidian canals by several authors (e.g., Galton, 1974a; Winkler et al., 1997; Norman, 1980), and may have also carried the palatine branch of the facial nerve (CN VII).

The basipterygoid processes lie just anterior to the foramina and extend below the rest of the braincase (Figures 9, 35). Each process is thin, retaining the same axial width until, distally, it flares slightly before ending bluntly, and articulates with the pterygoid in a peg-and-socket joint between its medial and quadratic processes (Figure 1).
Behind the ventral openings of the internal carotid foramina, occluding a line traveling directly out from them, are the basal tubera (Figures 6, 34, 35). These are paired arcs in posteroventral aspect that meet in a small median groove (Figure 6). The tubera are wider above and come to a point below, making a sharp ridge along their lower edge (Figure 35). There is a shallow groove running up the posterior side of the tubera, which continues up the cristae tuberales onto the prootic (Figure 35 ). The tubera of all other ornithopods are described as 


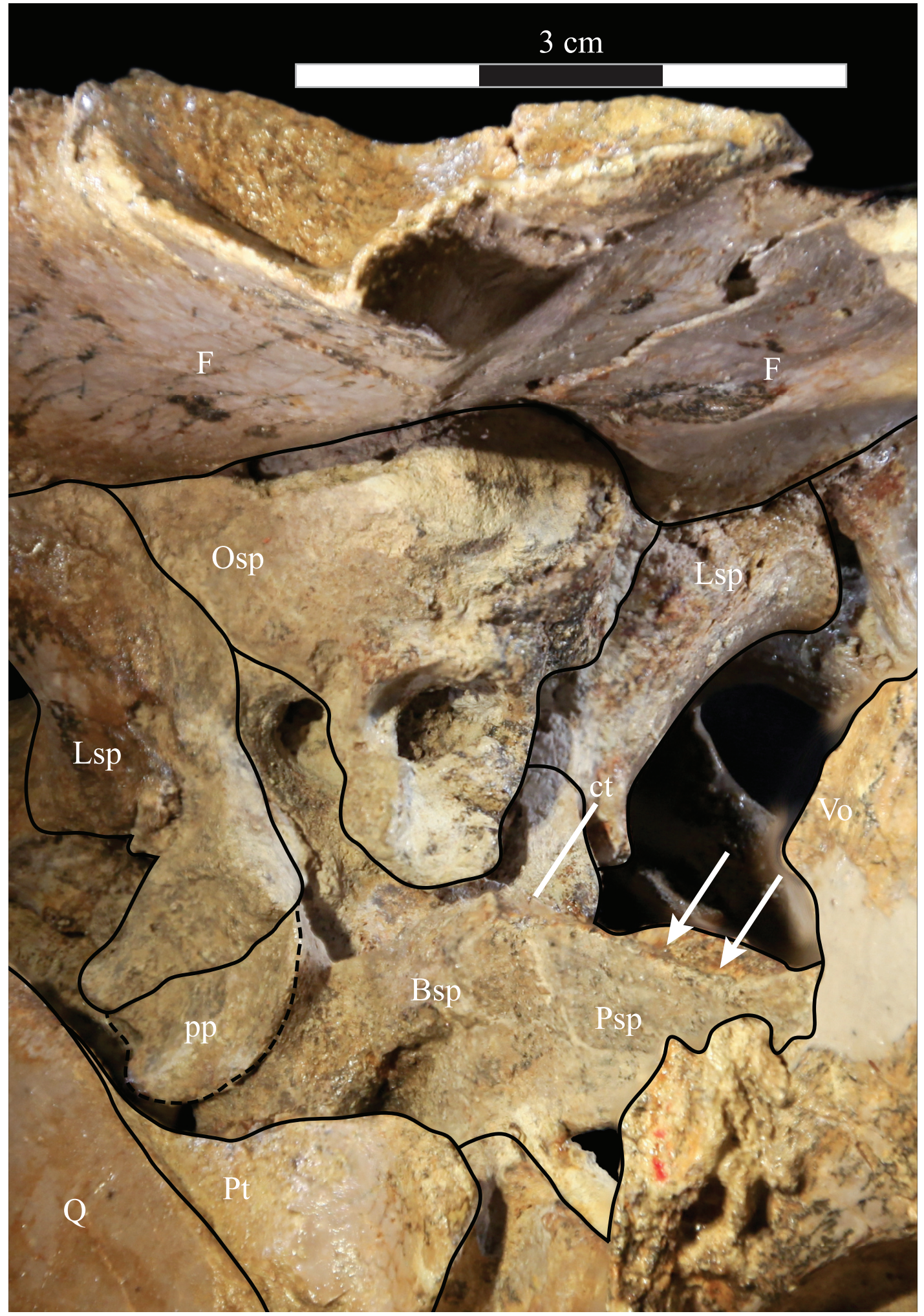

FIGURE 43. Photograph of the parasphenoid and basisphenoid complex and the anterior braincase of OMNH 58340. The parasphenoid extends forward (right) from the body of the basisphenoid and, at this angle, disappears behind the displaced right posterior process of the vomer. The trench running along the dorsal surface of the parasphenoid is visible (indicated by arrows). The ventral outline of the preotic pendant is indicated by the dashed line. Anterior is to the right and out of the page. Abbreviations: ct - possible crista trabecularis; pp - preotic pendant. 
bulbous and are more poorly ossified or defined (e.g., Ouranosaurus nigeriensis).

The cristae tuberales are narrow and long and continue back onto the prootics from the basal tubera (Figure 35). Between the paired cristae on the posterior face of the basisphenoid (Appendix 4: Basisphenoid) is a flat surface with a small median tubercle. Just above the tubercle are two small foramina for the abducens nerves (CN VI), which continue forward to exit the bone near the hypophyseal fossa (Figures 32, 34). Above the tubercle, within the braincase, is a small fossa that corresponds roughly to the pontine flexure of the endocast (Figure 36, Appendix 4: Basisphenoid).

The right side of the basisphenoid where these foramina emerge is not entirely preserved, and the exact path of the right foramen is indeterminate. The left foramen continues outside the fossa to emerge without entering it, while the right emerges from broken bone behind this point, appearing to pass through the fossa. However, because this area is incompletely preserved, it is possible that the left and right paths are more similar.

On either side of the hypophyseal fossa are two short processes, the preotic pendants (Figures $34,35,43$ ), composed of the basisphenoid and laterosphenoid (Figures 35, 43), contra Galton (1983; 1989), who assigned the dorsal component to the prootic. The plateau-like lateral surface of each pendant is axially broad, with all but its dorsal edges undercut. According to Ostrom (1961), the undercutting probably transmitted at least one of the branches of the trigeminal nerve ( $\mathrm{CN} \mathrm{V})$, either the maxillary $\left(\mathrm{V}_{2}\right)$ or both the maxillary and mandibular $\left(\mathrm{V}_{3}\right)$ branches (Figures 35,43$)$. The positioning of the trigeminal foramen in relation to the pendant, however, may indicate that the mandibular branch traveled down the rear of the basipterygoid process, or close to it (Figure 35). Ostrom also designated the lateral surface of the pendant as the origin of $M$. levator bulbi. However, Norman (1984) and Galton (1989) both later identified it as the origin of components of the constrictor dorsalis musculature.

The basisphenoid articulates across the preotic pendants with the laterosphenoid above in an undulating line on the left side (Figure 35) and in a straighter line on the right (Figures 9, 43). The articulation of the laterosphenoid with the basisphenoid continues up to cover the dorsal surface of the latter (Figure 42). The laterosphenoids also bridge the gaps between the main body of the basisphenoid and its cristae tuberales behind, which meet the laterosphenoids medially (Figure 35 ). The basisphenoid flatly articulates with the basioccipital between the cristae tuberales. The cristae of the basisphenoid abut the corresponding components of the prootics above (Figure 35).

The posterior processes of the laterosphenoids largely exclude the basisphenoid from the floor of the endocast (Figure 42). However, the state of preservation makes it difficult to tell whether or not the basisphenoid may contribute in small, irregular spaces between the laterosphenoids. The most likely contribution of the basisphenoid to the braincase is in a small gap between the laterosphenoids above the posterior processes and the pontine flexure (Figure 42).

Laterosphenoid. The laterosphenoids form two buttresses, supporting the skull roof (Figures 34, 43). Each laterosphenoid tapers above into its blunt, rounded capitate process that abuts the body of the postorbital laterally and the underside of the frontals and the lateral processes of the parietal (Figures 9, 34, Character 15:0). The suture with the parietal continues back along the medial surface of the superior temporal fenestra as the capitate process extends as a relatively flat, narrow ridge (Figures 35, 39). The element ends almost vertically behind, overlapping the anterior process of the supraoccipital (Figures 9, 35, 39). The external surface of the laterosphenoid between the buttress and the supraoccipital is laterally concave (Figure 35).

The posterior border of the laterosphenoid continues down until it hits the prootic, at which point it angles forward to run along the crista prootica (Figure 35). Just below the crista, the element forms all but the posterior borders of the trigeminal foramen ( $\mathrm{CN} V$ ), which probably carried the medial cerebral vein as well (Oelrich, 1956). There is a deep, partially roofed groove (Figures 34,35 ) running forward from the foramen that likely carried the ophthalmic branch $\left(V_{1}\right)$ of the trigeminal nerve (Ostrom, 1961).

Behind the lower margin of the trigeminal foramen, the laterosphenoid forms a small process that bridges the body and crista tuberalis of the basisphenoid and meets the ventral process of the prootic. There is an additional ridge in front of the crista that is begun on the prootic, which runs farther forward to become the posterior border of the preotic pendant and then angles down to become the posterior margin of the basipterygoid process of the basisphenoid (Figure 35). Galton (1989) identified the dorsal half of the preotic pendant in 
Tenontosaurus as the prootic; CT data from OMNH 58340 clearly show otherwise.

The pendant forms both the lower border of the laterosphenoid and part of the outer wall of the sella turcica (Figure 34). Just above the sella, the laterosphenoid forms the posterior half of the border of the oculomotor foramen (CN III). Above this, the base of the capitate process overlaps the posterior process of the orbitosphenoid (Figures 39, 42 ). The leading edge of the bone is laterally displaced, presumably providing space for the bulge of the cerebrum (Figures 34, 39).

The laterosphenoids meet on the midline below to form the posterior and ventral borders of the infundibulum (Figures 39, 42). Behind this, the laterosphenoid runs back, forming part of the floor of the braincase beginning at the pontine flexure. Here, the abducens foramen (CN VI) pierces the element and continues straight forward to the basisphenoid. Although not well preserved in this area, the paired laterosphenoids probably met along the midline beginning at the infundibulum and continued back to the basioccipital.

Prootic. The prootic is made of two main components: a posterior and a ventral process (Figure 35 ). The posterior process has a convex dorsolateral surface and tapers in height and width as it overlaps the opisthotic behind. The crista prootica runs below this, continuing back from the laterosphenoid to meet the paroccipital process of the opisthotic (Figure 34, 35, Character 50:0). Tenontosaurus tilletti shares a similar composition of the crista prootica with both basal ornithopods and basal iguanodontians, while in Ouranosaurus nigeriensis and hadrosaurs the ridge is restricted to the prootic and opisthotic (Appendix 3). In life, based on the orientation of the lateral semicircular canals, the crista was probably held roughly horizontal (Figure 32) in the "alert" head posture (Witmer and Ridgely, 2009).

Two more ridges extend forward from the middle of the crista prootica: an anterior, unnamed ridge and the crista tuberalis, which continue down to the preotic pendant and the basal tubera of the basisphenoid, respectively (Figure 35 ). The foramen for the facial nerve (CN VII) emerges between these two ridges as it does in virtually all other ornithopods.

Just behind the crista tuberalis is the fenestra ovalis, which emerges entirely from the prootic (Figure 35). In most descriptions of ornithopods, the fenestra is noted to occur at the boundary between the prootic and opisthotic (e.g., Galton, 1974a; Norman, 1980). Although this is not pre- cisely the case in Tenontosaurus tilletti, the prominent stapedial recess is shared between the elements (Figure 35).

Behind the fenestra ovalis, within the stapedial recess, are the crista interfenestralis and the fenestra metotica (Figure 35). Behind the fenestra metotica is another ridge, the crista metotica, formed by the prootic and opisthotic. Behind this ridge is another large foramen (CN X), which briefly contacts the prootic internally (Figure 36 ). The remaining borders of this foramen are made up of the opisthotic. Although the fenestra ovalis almost certainly conveyed the footplate of the stapes (the fenestra opens into the middle portion of the lagena internally), which is not preserved, the contents of the remaining foramina are uncertain (see description of the cranial nerves below).

Below these foramina, the prootic tapers irregularly (Figure 35), articulating with the basisphenoid along most of its lower edge. The prootic is overlapped internally by the basioccipital (Figure 42). Behind the pontine flexure, there appears to be a small projection (which may actually be composed of matrix; it is difficult to determine in the CT images) extending inward from the right prootic to floor part of the braincase (Figure 42).

The prootic forms the posterior border of the large trigeminal foramen $(\mathrm{CN} \mathrm{V})$, which is completed by the laterosphenoid (Figure 35). Above this foramen, the prootic ends bluntly in front where it is overlapped by the laterosphenoid.

Beginning at the trigeminal foramen, there is a furrow running down the medial prootic that creates a corresponding ridge on the endocast. Above this groove, there is a small foramen that perforates the thin medial wall of the prootic to enter the endosseous labyrinth just below the anterior ampulla and a second foramen behind it, which enters the vestibule. These foramina probably carried the dorsal and ventral rami of the acoustic nerve (CN VIII, Figure 36, see description of cranial nerves below). Behind these foramina is a third, which enters the vestibule just before the common crus. The borders of this foramen are shared by the ventral processes of the supraoccipital and the prootic. Immediately before and partially confluent with the foramen of the dorsal ramus of the acoustic nerve, the canal for the facial nerve (CN VII) proceeds outward to emerge as described above. The remainder of this surface is fairly flat and angled forward so that the endocast expands out toward the trigeminal nerve and the pontine flexure. 
The prootic and the ventral process of the supraoccipital form an auditory bulla around the endosseous labyrinth (Figure 37). The anterior semicircular canal and its ampulla are contained primarily within the prootic (Figure 38). The anterior half of the lateral canal also travels through the prootic. Thescelosaurus neglectus shares these characteristics with Tenontosaurus tilletti. The element may wrap around the bottom of the common crus, but the CT image is unclear in this area. Aside from its ventral tip, the lagena is entirely contained within the prootic.

The anterior half of the deep conical (subarcuate) fossa, which contained the floccular lobe of the cerebellum, is found along the posterodorsal border of the prootic. The supraoccipital makes up the posterior half of the border where its ventral processes cover the prootic medially (Figures 37,41 ). Hypsilophodon foxii and Parksosaurus warreni have a similarly pronounced conical fossa, while that found in Thescelosaurus neglectus is shallower (Galton, 1989).

Orbitosphenoid. The orbitosphenoid is perhaps the most poorly preserved element in $\mathrm{OMNH}$ 58340 , due partially to taphonomic preservation, over-preparation, and possibly to poor or incomplete ossification. It is a simple, thin bone; almost exclusively an anteriorly convex dome that extends back into posterior processes (Figures 34, 35, 39, Appendix 4: Orbitosphenoid).

In closely related taxa, such as Tenontosaurus dossi, the domelike orbitosphenoid appears to resemble that in T. tilletti quite closely. In ornithopods more derived than T. tilletti (Figure 7), especially those in which an ossified presphenoid is preserved (e.g., Ouranosaurus nigeriensis, Corythosaurus casuarius), the orbitosphenoid appears more conical, terminating in an anterior keel or point. The presphenoid is not preserved in either $T$. dossi or T. tilletti, either because of poor preservation or lack of ossification, but may have completed the pointed profile found in higher taxa.

The orbitosphenoid is perforated by numerous foramina (Figure 34). Dorsally, on either side, are two small holes, probably for the trochlear nerves (CN IV). The element narrows forward into the fairly large, median olfactory foramen (CN I), just below its articulation with the frontals. The orientation of the orbitosphenoid and of the arcuate ridges of the frontals may indicate that neural tissue or an extension of the braincase (probably the presphenoid) continued forward between the ridges (Figure 21).
Below the olfactory foramen is another, slightly smaller foramen (Figure 34), which, because of its association with the hypophyseal complex, was likely for the optic nerves (CN II). Due to preservation, the exact nature of this foramen is indeterminate. A small median tubercle here may indicate that a pair of foramina were present, possibly relating to the optic chiasm.

On either side of this foramen are a pair of tall oculomotor foramina (CN III), bordered laterally by the laterosphenoids (Figure 34). The laterosphenoids continue above to overlap the posterior processes of the orbitosphenoid, which appear wedged between the laterosphenoids and the endocast (Figure 39).

Below the optic foramen, the orbitosphenoid begins to narrow until it divides into two small tubercles over the hypophyseal fossa (Figure 34). These may be remnants of an articulation with the parasphenoid, presphenoid, or with soft tissue. The infundibulum would have traveled below and then behind these tubercles on its way to the brain (Figure 39). The orbitosphenoid hangs out over empty space and does not articulate with any other element below, although this may be due to a lack of preservation or over-preparation.

As was stated, the orbitosphenoid of Tenontosaurus tilletti closely resembles that of $T$. dossi in most respects, with the spacing and relative positioning of the foramina correlating very closely (Winkler et al., 1997, figure 10). However, the orbitosphenoids of $T$. dossi are paired and display a generally looser configuration, with larger foramina and a lesser degree of fusion among the elements (e.g., the oculomotor and trochlear foramina appear to be linked). This may represent a difference in relative maturity or a phylogenetic difference between the specimen of $T$. dossi figured and the specimen of $T$. tilletti described here. In light of the fact that the $T$. dossi specimen is larger, the latter possibility seems more likely.

Endocast. In keeping with common practice (e.g., Ostrom, 1961; Galton 1989), the following description is made with the assumption that the endocast approximates the shape of the brain in life. The true form of the brain itself is likely largely obscured by the thick dura mater present in most reptiles.

The endocast of OMNH 58340 appears triangular in lateral aspect (Figure 44.1), with a roughly $90^{\circ}$ angle in the dorsal sagittal sinus and its hypotenuse running from the foramen magnum to the olfactory foramen $(\mathrm{CN} \mathrm{I})$. The hypotenuse is fairly flat due to the lack of a pronounced pontine flexure (Figure 44.1). The dorsally expanded cerebrum 
and the exit of the medulla through the foramen magnum obscure the triangular profile (Figure 44.1). The endocast of OMNH 58340 differs from the elongate specimens of Tenontosaurus tilletti from the Cloverly Formation of Montana by Galton (1989, text-figure 4) and instead more closely resembles Dryosaurus altus and Hypsilophodon foxii in general form (Galton, 1989, text-figure 1). The endocranium of hadrosaurs tends to be more elongate as well (Ostrom, 1961).

Three constrictions are apparent in anteroventral aspect, separating some of the major sections of the brain: one at the front of the endocranial cavity, where the forebrain sends out the olfactory tracts, a second between the telencephalon (cerebrum) in front and the diencephalon and mesencephalon (cerebellum) in the middle, and a final constriction, filled with the endosseous labyrinths (Figure 32), between the mesencephaIon and the rhombencephalon (Figure 44.2). There are two faint lobes at the top of the endocast with a faint ridge in between (Figure 44.3). These hemispheric lobes, which are not present in other ornithopods, are consistent with the observations of Galton (1989) for Tenontosaurus tilletti.

The rhombencephalon, or hindbrain, of Tenontosaurus tilletti comprises the endocast forward from the foramen magnum to include the cerebellum (Figures 44.1, 44.2). It is composed of the myelencephalon behind and the more anterior metencephalon.

The myelencephalon begins at the foramen magnum and ends at the foramen for the glossopharyngeal nerve (CN IX) and the constriction created by the endosseous labyrinths (Figures 44.1, 44.2). It was composed of the medulla in life and likely gave rise to the final five cranial nerves (CN VIII-XII). There are two small projections (Figure 44.4) pointing outward above the hypoglossal nerves (CN XII), which Witmer and Ridgely (2009) interpreted in Tyrannosaurus rex as blind dorsal venous sinuses. Above these projections, the surface of the endocast forms a cone pointing to the dorsal sagittal sinus (Figure 44.4), which is bordered by the endosseous labyrinth constriction. The region above the medulla may have been filled with the choroid plexus (Ostrom, 1961).

The metencephalic portion of the rhombencephalon includes the cerebellum and likely gave rise to the fifth through seventh cranial nerves, as well as part of the eighth (CN V-VIII). The prominent bulbous expansions below the dorsal sagittal sinus (Figure 44.4) may preserve the outline of the cerebellum. These expansions have also been interpreted as endolymphatic sacs (Ostrom, 1961). The endocast narrows into the dorsal sagittal sinus above here (Figures 44.1, 44.4). Galton (1989) claimed that this space, which he restores between the parietal and supraoccipital, was probably filled with cartilage in life. In OMNH 58340 this area is bounded entirely by the supraoccipital. An anteroventral ridge (Figure 44.1) runs along the suture of the supraoccipital and prootic behind and the parietal and laterosphenoid ahead, which ends in the trigeminal nerve (CN V, Appendix 4: Cranial Nerves, Endocast). An anterodorsal ridge (Figure 44.1) runs down from the area around the facial and acoustic nerves (CN VII, VIII) to end near the trigeminal nerve. The flocculus projects back from between these ridges, through the arc of the anterior semicircular canal (Figures 32, 44.1, Appendix 4: Endocast, Endosseous Labyrinths). There is no flocculus present in endocasts of hadrosaurs (Langston, 1960; Ostrom, 1961). An additional ridge runs along the underside of the endocast from the foramen magnum to the abducens nerves $(\mathrm{CN} \mathrm{VI})$ and may have carried the palatine arteries (Figure 44.2).

The mesencephalon, or midbrain, is poorly defined in this specimen, as it is in most ornithopods (Ostrom, 1961; Galton, 1989). The poor definition is likely due to the overhang of the enlarged cerebrum above. The oculomotor and trochlear nerves (CN III and IV) probably originated from this region. The mesencephalon includes the tectum and tegmentum. The former is developed into the optic lobes in reptiles and birds, which are not visible in $\mathrm{OMNH} 58340$ (e.g., Witmer and Ridgely, 2009, figure 4).

The prosencephalon, or forebrain, comprises the diencephalon behind and the telencephalon in front. The prosencephalon is located between the second constriction, in the region of the trigeminal nerves, and the first, just before the branching of the olfactory tracts (Figure 44.1).

The diencephalon probably formed most of the constricted region behind the expanded cerebrum, and shared this space with the mesencephalon. The main features of this region of the brain are the infundibulum below and a small dorsal projection, which may represent parietal or pineal organs (Figure 44.1). The optic nerves (CN II) likely arose from this area of the brain as well. The infundibulum extends down from the endocast under the optic nerves (Appendix 4: Cranial Nerves, Endocast), behind the lower orbitosphenoid, and between the laterosphenoids and abducens nerves (Figures 34, 39, 42). The infundibulum probably 


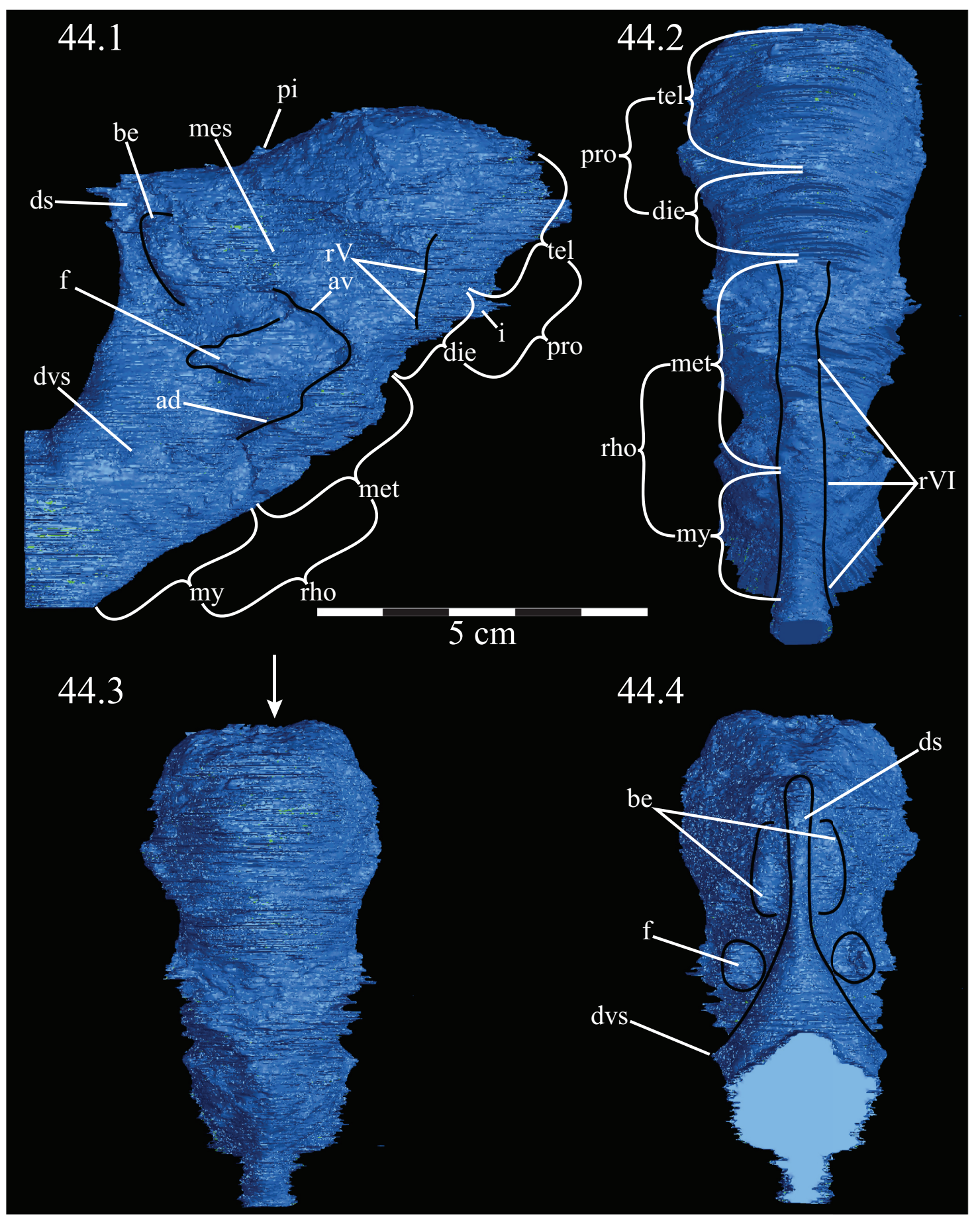

FIGURE 44. Right lateral (44.1), anteroventral (44.2), anterior (44.3), and posterior (44.4) views of the virtual endocast of OMNH 58340. 44.1 shows various landmarks for the passage of venous sinuses and cranial nerves. 44.2 highlights the waists between the cerebrum and cerebellum anterodorsally and the cerebellum and medulla posteroventrally. 44.3 shows the faint lobes present on the dorsal surface of the endocast, separated by a small trough (indicated by arrow). 44.4 shows the dorsal sagittal sinus in the middle and the flocculi of the endocast, as well as the dorsal bulbous expansions. Abbreviations: ad - anterodorsal ridge; av - anteroventral ridge; be - bulbous expansions; die - diencephalon; ds - dorsal sagittal sinus; dvs - dorsal venous sinus; f - flocculus; i - beginning of infundibular stalk; mes - mesencephalon; met - metencephalon; my - myelencephalon; pi - pineal process; pro - prosencephalon; rho - rhombencephalon; rV - ridge running to trigeminal nerve (CN V); rVI - ridge running to abducens nerves (CN VI) tel - telencephalon. 
expanded forward into the pituitary gland below the orbitosphenoid, and would have resided in the sella turcica below. The sella divides below into two canals that carried the internal carotid arteries. The constriction of the anterior sella suggests that the pituitary did not descend very deeply into it; the combined bores of the internal carotid canals (and, presumably, their contents) nearly equal the circumference of the space. If the gland did not descend far into the sella, the position of the abducens nerves as they pass the hypophyseal fossa (Figure 32, Appendix 4: Cranial Nerves, Endocast) serves as a good indicator of the lower pituitary.

The cerebrum comprises the entire telencephalon, which is the front of the endocast and its widest point (Figures 44.2, 44.3). The telencephalon contains the expanded hemispherical lobes (Figure 44.3). Galton (1989) could not determine the anterior extent of this region due to poor preservation of the orbitosphenoid. Although this element is again poorly preserved in OMNH 58340, it provides sufficient detail to determine the anterior border of the endocast (Figure 44.1). The telencephalon terminates in the olfactory tracts $(\mathrm{CN} \mathrm{I})$, which run along the underside of the paired frontals (Figure 21).

Cranial Nerves. The paths of the cranial nerves of OMNH 58340 are generally distinguishable (Figure 35). The first eight foramina are readily identifiable (Figures 34, 35, 42), and there are a sufficient number of foramina for the remaining nerves, although their actual contents are uncertain (Figure 35). Tenontosaurus tilletti most closely resembles $T$. dossi in the distribution of its foramina, especially in the first six, which are well preserved in both taxa. In ornithopods more derived than Iguanodon bernissartensis (Figure 7, e.g., Ouranosaurus nigeriensis, Corythosaurus casuarius), the contributions of the ossified presphenoid alter the configuration of the first six foramina. The remaining nerves (CN VII-XII) are fairly consistent throughout Ornithopoda (e.g., Hypsilophodon foxii, Edmontosaurus regalis).

The olfactory nerves $(\mathrm{CN} \mathrm{I})$ originated in the telencephalon and exited the braincase via a foramen bordered by the frontals above and on all other sides by the orbitosphenoid (Figures 32, 34). Because of the very smooth underside of the frontals, it is likely that an anterior extension of neural tissue, probably composed of olfactory tracts, nerves, bulbs, or epithelium extended forward, rather than osseous elements, which would have likely left a trace on the neighboring elements (Figures 21,43 ). In ornithopods more derived than Iguanodon bernissartensis (Figure 7, e.g., Ourano- saurus nigeriensis, Corythosaurus casuarius) the ossified presphenoid continues the neural path forward. The only fossilized anterior border for the olfactory apparatus in OMNH 58340 is the termination of the arcuate ridge at the ventral process of the prefrontal (Figure 21).

The optic nerves (CN II) exited the braincase via a smaller foramen in the orbitosphenoid below the olfactory foramen, having arisen from the diencephalon (Figure 34). Due to the state of preservation of the element, it is impossible to tell if this foramen was originally divided, and the optic chiasm is unknown. An ossified chiasm is preserved in hadrosaurs. Based upon the relative positioning of the orbital fenestrae and the exit of the optic nerves from the braincase, it is likely that the eyes were probably set far back and just above the middle of the orbit (Figure 2).

The oculomotor foramina (CN III) exit on either side of the optic foramen (Figures 34, 42). The nerves probably arose from the ventrolateral surface of the mesencephalon. The foramina are tall, although not nearly as large as those found in Tenontosaurus dossi, owing to the looser configuration of the neurocranial elements present in that taxon (Winkler et al., 1997, figure 9). These foramina are bordered by the laterosphenoids laterally and by the orbitosphenoid on their other borders (Figures 34, 35).

The trochlear foramina (CN IV) are located above the oculomotor foramina, nearly on a level with the olfactory foramen, and their nerves probably arose from the ventral mesencephalon (Figures 34,42 ). These foramina are quite small and are in line with the rear corners of the orbits. This nerve is solely responsible for the innervation of $M$. obliquus oculi superioris.

The trigeminal nerves ( $\mathrm{CN} \mathrm{V}$ ) arose from the metencephalon and exited from large foramina bordered by the prootics posteriorly and the laterosphenoids on all other sides (Figure 35). The foramina are large enough to have accommodated the trigeminal ganglia. Deep, partially enclosed grooves run forward from the foramina, which carried the trigeminal ophthalmic branches $\left(\mathrm{V}_{1}\right)$. The other two branches of the trigeminal nerve, the maxillary and the mandibular branches $\left(V_{2}\right.$ and $V_{3}$ ), likely extended farther down before they separated. There is another pronounced groove, just below the preotic pendant, which possibly carried the maxillary branch (Figure 35). There is no obvious feature preserved on the skull for the passage of the mandibular branch, but if it followed the maxillary branch down to the pendant, it is likely that it 
continued behind the basipterygoid process of the basisphenoid for a short space before it moved out to the mandible.

The foramina for the abducens nerves ( $\mathrm{CN} \mathrm{VI)}$ begin at the floor of the metencephalon and extend forward through thin medial pieces of the laterosphenoids. The foramina continue forward through the body of the basisphenoid until they exit lateral to the hypophyseal fossa (Figure 34). The state of preservation of the basisphenoid here makes the exact paths of these foramina difficult to distinguish (see description of the parasphenoid/basisphenoid above). There is a groove present in the lateral walls of the fossa for the passage of these nerves in Thescelosaurus neglectus (Galton, 1989).

The paths of the facial and acoustic nerves (CN VII and VIII) are similar to those previously described in other reptiles (Oelrich, 1956) and ornithopods (Ostrom, 1961; Galton, 1989). Both nerves probably originated in the metencephalon. There are three small foramina which enter the inner ears: one just below the ampulla of the anterior semicircular canal and another into the vestibule, both of which travel through the medial wall of the prootic; and the final one just before the common crus of the canals, the borders of which are split between the prootic and the ventral process of the supraoccipital (Figures 42, 45.1). The first of these foramina divides into two branches, which may represent two openings internally, although they are not preserved separately in OMNH 58340. As in many other ornithopod taxa, the anterior of these branches carried the facial nerve out to exit forward of the crista tuberalis of the prootic (Figure 35). The shorter, posterior branch ends just below the anterior ampulla of the endosseous labyrinth and conveyed the dorsal branch of the anterior ramus of the acoustic nerve. The middle foramen hits near the vestibule and carried the ventral branch of the anterior ramus. The final foramen possibly carried the posterior ramus of the acoustic nerve, as described by Galton (1989, Dryosaurus altus, plate 2, figure 6, and Thescelosaurus neglectus, text-figure 4K). Witmer and Ridgely (2009) identified this foramen as carrying the endolymphatic duct in theropods.

The exact paths of the glossopharyngeal, vagus, and spinal accessory nerves (CN IX-XI) are difficult to determine. These nerves all likely originated in the myelencephalon. There are four candidates for the passage of these nerves first, the fenestra metotica; second, a foramen of similar size posterior to the fenestra metotica, just behind the crista metotica; and finally, two smaller foram- ina below, near the border of the opisthotic with the basioccipital (Figure 35). Witmer and Ridgely (2009) and Knoll et al. (2012) assign the glossopharyngeal nerve to the fenestra metotica immediately behind the fenestra ovalis, which necessitates close association between the vestibule of the ear and the glossopharyngeal nerve, as revealed by CT data here. Various other authors (e.g., Galton, 1974a; Winkler et al., 1997) assign the above nerves, with the internal jugular vein, to these foramina in different combinations. The internal jugular vein likely occupied the first of the smaller foramina, which traces a sinuous path through the opisthotic (Appendix 4: Cranial Nerves, Exoccipital/Opisthotic). In addition to the indirect course of this opening, its presence seems to be the most variable between specimens, as noted by Ostrom (1961). The foramen immediately behind this one takes a much more direct course out of the cranium, and likely accommodated the spinal accessory nerve. It is possible that this posterior foramen carried an anterior branch of the 12th nerve, described below.

The hypoglossal nerve (CN XII), which probably originated in the myelencephalon, exited the braincase from the last of the cranial foramina and, possibly, from the opening immediately before it (Figure 35 ). The posterior hypoglossal foramen is one of the larger foramina and takes a direct course through what is likely the exoccipital (rather than the opisthotic). This foramen is large enough to have accommodated vasculature as well.

Endosseous Labyrinths. The endosseous labyrinths of OMNH 58340 comprise the lateral, anterior, and posterior semicircular canals, and the cavum capsularis, which includes the vestibule above and the lagenar recess below (Figures 45.2, 45.3).

The largest of the semicircular canals is the anterior. Interestingly, the posterior canal is larger in hadrosaurs (Brown, 1914; Langston, 1960). In addition, the general form of the inner ear of Tenontosaurus tilletti is much more gracile than that found in hadrosaurs, in that the canals tend to be thinner, while the common crus is certainly of lesser girth.

In fact, the general form of the endosseous labyrinths of Tenontosaurus tilletti more closely resembles those of large theropods (e.g., Witmer and Ridgely, 2009, figure 8; Eddy and Clark, 2011, figure 17). Witmer and Ridgely (2009) noted that the well-developed semicircular canals in Tyrannosaurus rex and other theropods, especially the elongate anterior and lateral canals, form a close 


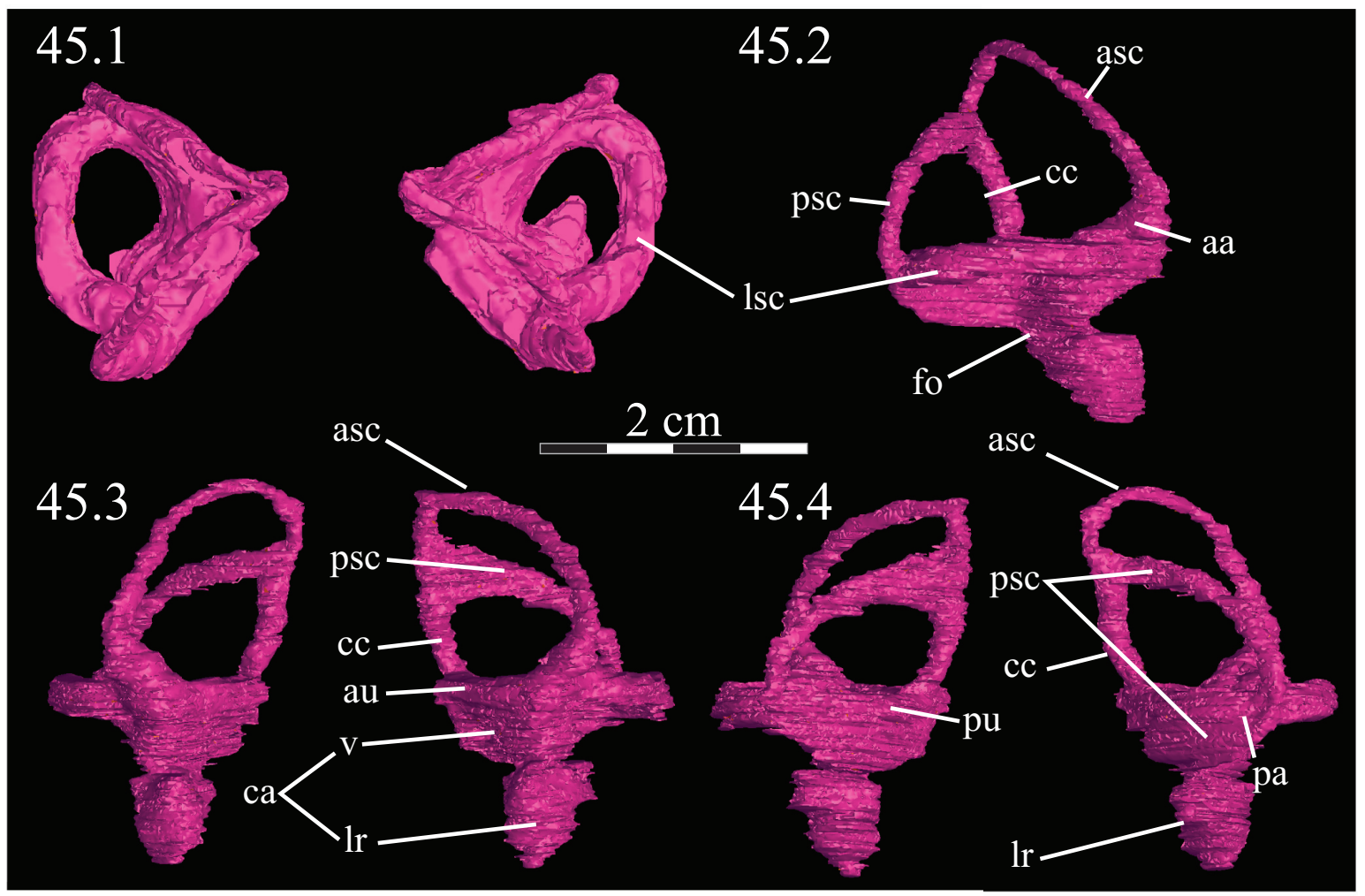

FIGURE 45. Dorsal (45.1), right lateral (45.2), anterior (45.3), and posterior (45.4) views of the virtual endosseous labyrinths of OMNH 58340. The canals are oriented in 45.1 such that anterior is above and posterior below. Ventral is down in all other images. Abbreviations: aa - anterior ampulla; asc - anterior semicircular canal; au - anterior utricle; ca - cavum capsularis; cc - common crus; fo - fenestra ovalis; Ir - lagenar recess; Isc - lateral semicircular canal; pa - posterior ampulla; psc - posterior semicircular canal; pu - posterior utricle; v - vestibule.

functional relationship with the eye muscles utilized by the vestibulo-ocular reflex (Schwarz and Tomlinson, 1994; Cohen and Raphan, 2004; Graf and Klam, 2006). The vestibulo-ocular reflex is associated with coordinating movements of the eye muscles so that, during motion of the head, a focused image remains in the eye. This evidence supports the conclusion of Forster (1990), based on aspects of the postcranial skeleton, that $T$. tilletti was bipedal. The endosseous labyrinths of basal ornithopods and other iguanodontians are currently unknown.

Each canal is distally flattened to varying degrees rather than being perfectly round (Figures $45.1,45.2)$. There is an angle of roughly $60^{\circ}$ between the anterior and posterior canals in dorsal aspect, similar to that measured in hadrosaurs (Langston, 1960). Between the anterior and lateral canals there is an angle of about $75^{\circ}$, and an angle of about $90^{\circ}$ between the posterior and lateral canals. The angle of the lateral canal indicates that the 'alert' position of the skull of Tenontosaurus tilletti was such that the crista prootica and the dorsal surface of the parietal were held nearly parallel to the ground (Figure 32).

The anterior canal passes through the supraoccipital dorsally and the prootic ventrally (Appendix 4: Endosseous Labyrinths, Prootic, Supraoccipital), while the flocculus of the endocranium interjects into the midst of the labyrinth under the arc of the anterior canal. The lateral canal is divided between the prootic in front and the opisthotic behind (Appendix 4: Endosseous Labyrinths, Opisthotic, Prootic). The ventral process of the supraoccipital surrounds the common crus. The posterior canal passes mainly through the opisthotic, although a small portion enters the supraoccipital as it joins the common crus, well below the anterior canal (Figure 45.2, Appendix 4: Endosseous Labyrinths, Opisthotic, Supraoccipital).

The anterior ampulla is readily visible where the anterior and lateral canals meet, but the lateral ampulla is more difficult to discern (Figure 45.3, Appendix 4: Endosseous Labyrinths). The posterior ampulla is very difficult to see with any certainty (Figure 45.4). It is possible to trace the path of the 
posterior canal as it passes the lateral canal ventromedially (Figure 45.4), without creating an inferior common crus, as it does in crocodilians (Wever, 1978). This is a shared characteristic with some theropods (Sanders and Smith, 2005).

The vestibule of the cavum capsularis does not contain a dramatically expanded space for an enlarged sacculus, as it does in extant squamates (e.g., Oelrich, 1956). Rather, the vestibule begins below the lateral canal and extends down (Figures $45.2,45.4)$. Otoliths were not found. This configuration is similar to that reported in hadrosaurs (Brown, 1914; Langston, 1960).

The utriculi are faintly discernible extending along the vestibules in front of and behind the bases of the common crura. Traces of the utriculi are much more pronounced in the hadrosaur Lophorhothon atopus (Langston, 1960). The fenestra ovalis opens on the anterolateral vestibule, just above a constriction that signals the beginning of the lagenar recess (Figure 45.2).

As discussed in the section on cranial nerves above, there are three foramina on the medial surface of the vestibule that communicate with the endocranial space. One of these, the middle foramen, is situated on the medial side of the constriction mentioned above. Another foramen, in front of the former, enters the endosseous labyrinth just below the anterior ampulla, in a space that may have held the anterior utriculus. These likely carried the ventral and dorsal rami of the acoustic nerve (CN VIII) into the vestibule, the space of the inner ear largely responsible for auditory perception. The smallest of the three foramina is located at the base of the common crus. Witmer and Ridgely (2009) interpreted this foramen as carrying an endolymphatic duct in certain theropods, although other authors (e.g., Oelrich, 1956, Galton, 1989) claimed that it transmitted the posterior ramus of the acoustic nerve.

After the initial constriction below the vestibule, the lagenar recess continues down (Figure 45.2). This constriction appears more pronounced than that described in hadrosaurs (Langston, 1960). The lagenar recess continues below, rotating slightly as it descends, until the tip of the lagena opens into the fenestra metotica (Figure 35). The tips of the lagenar recesses appear to be formed by the basioccipital.

\section{Mandibular Series}

The mandibles of OMNH 58340 are generally well preserved. The right mandible is partially crushed, dorsoventrally, but the left is virtually undistorted. The exact nature of a few of the elements, including the splenials, articulars, coronoids, and prearticulars, is uncertain. Both jaws were found in near-articulation with the skull and with each other. The predentary was found disarticulated from the jaws, associated with the postcrania. In an emendation to Ostrom (1970), the retroarticular process does not sweep dramatically upward at its posterior tip. Rather, the dorsal curve is gentle (Figures 46, 47).

Predentary. The median predentary is deep (Figure 48), contra Ostrom (1970). The element is $U$ shaped in dorsal aspect (Figure 48.1), as in many other iguanodontians (e.g., Tenontosaurus dossi, Mantellisaurus atherfieldensis), not pointed as in basal ornithopods and Zalmoxes robustus, or rectangular as in more derived ornithopods (Figure 7, e.g., Ouranosaurus nigeriensis, Corythosaurus casuarius). The element is composed of the body, which terminates behind in two dentary processes and a median ventral process (Figures 48.1, 48.2). A ventral sulcus runs along the underside of the body (Figure 48.3). This sulcus is the point of articulation with the symphysial processes of the dentaries, the symphysis of which was covered by the ventral process of the predentary. There are several foramina within the sulcus that correspond to similar ones on the symphysial processes of the dentaries (Figure 49.2). The predentary occluded with the beak of the premaxilla above. The element was recovered within the ribcage of OMNH 58340.

The sharp, edentulous dorsal rim of the predentary is lined with a series of denticles that form the anterior occlusal margin of the mandibles (Figures 48.2, 48.4, Character 62:1). This feature is present in all iguanodontians and hadrosaurs, with the exception of Dryosaurus altus and Zalmoxes robustus (Appendix 3). The largest, primary denticle lies on the midline. Two pairs of denticles flank the primary denticle, which are, in turn, flanked by about three more pairs of clustered, very small, bony tubercles. Together, this gives the impression of a median primary denticle, flanked by five pairs of distinct, progressively smaller units that extend nearly to the posterior edge of the bone (Figure 48.2-4). Several authors (e.g., Ostrom, 1970; Norman, 2004) have suggested that these denticles serve to anchor an overlying keratinous rhamphotheca and may correspond to similar external features.

The primary denticle is bordered on either side by two furrows running behind (Figure 48.4), which terminate below in two foramina, one of which pierces through to the anterior side of the 

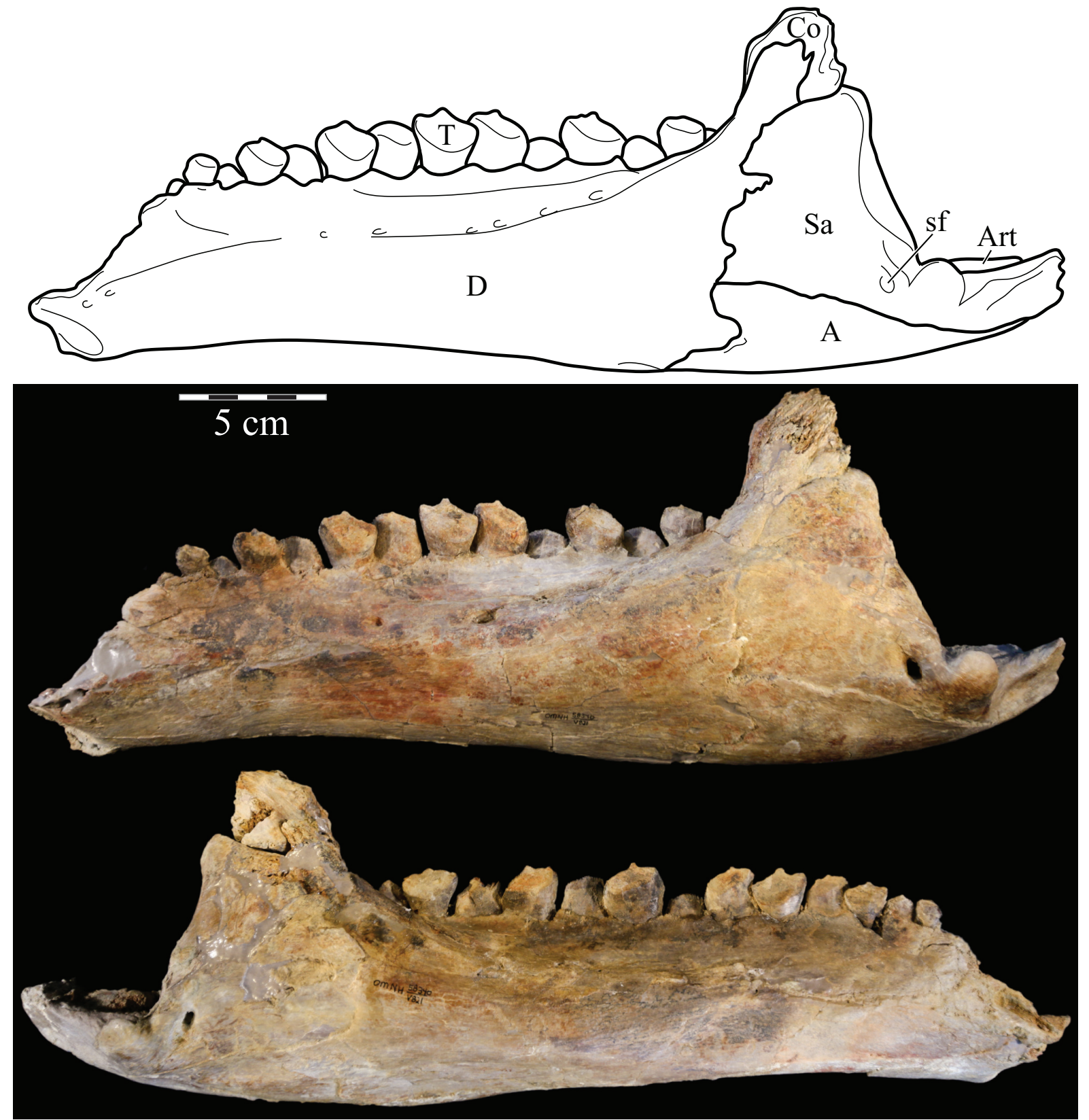

FIGURE 46. Lateral schematic of the left (above) and photographs of the left (middle) and right (below) mandibles of OMNH 58340. Note the amount of dorsoventral compression of the right mandible when compared with the left. Anterior is to the left in the first two images and to the right in the third. Abbreviation: sf - surangular foramen.

occlusal margin (this may be an artifact of preparation). A series of shrinking foramina run back from these larger foramina in an arc parallel to the occlusal margin (Figure 48.1). These presumably provided vascular and nutritive supply to the overlying beak (Norman, 1980). The medial posterior surface of the body of the predentary slopes down dramatically, but this slope becomes nearly horizontal as the dentary processes curve behind (Figure 48, Appendix 4: Predentary). The posterior surface of the body terminates below in a small median tab that forms part of the border of the ventral sulcus (Figures 48.3, 48.4).

The face of the predentary is rugose and covered in a series of furrows and ridges that run ventrolaterally, decrease in size laterally, and likely helped anchor the rhamphotheca (Figure 48.2). The larger, more medial furrows terminate in foramina below, which probably served a similar 


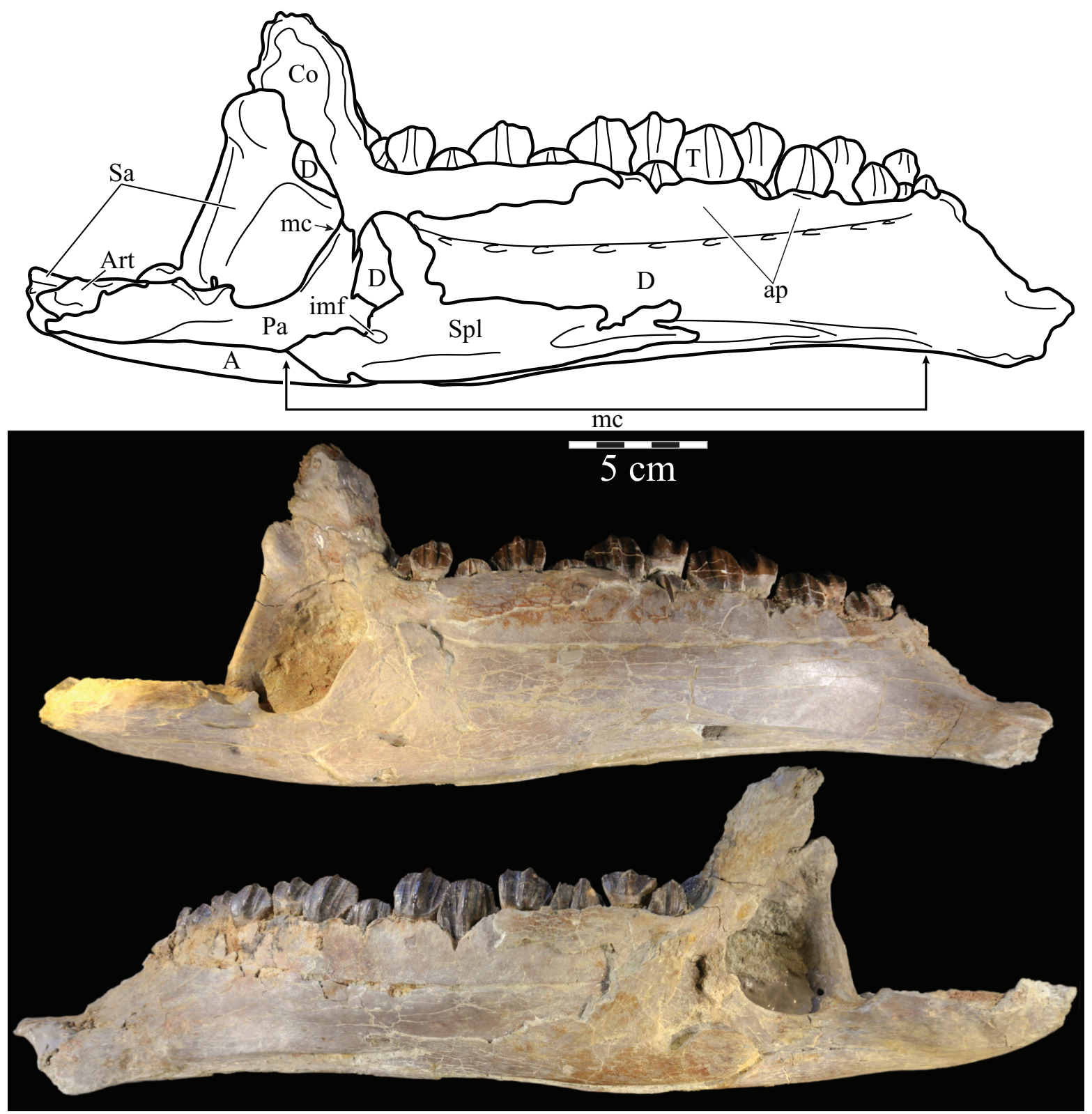

FIGURE 47. Medial schematic of the left (above) and photographs of the left (middle) and right (below) mandibles of $\mathrm{OMNH}$ 58340. Note the amount of dorsoventral compression of the right mandible when compared with the left. Anterior is to the right in the first two images and to the left in the third. Abbreviations: ap - alveolar parapet; imc - internal mandibular foramen; mc - Meckelian canal.

function to those mentioned above on the posterior surface.

The ventral process extends down from the anterior surface of the body (Figure 48.3). It is roughly as tall as the rest of the body above it. The process is not bifurcate in OMNH 58340 (Character 61:0, contra Sereno, 1986; Norman, 1990). However, a very shallow median indentation is present on the ventral edge (Figures 48.2, 48.4), only slightly more pronounced than that figured for Tenontosaurus dossi (Winkler et al., 1997, figure
10). The apparent variability of the ventral process in Ornithopoda may be analogous to the cleft chin in humans, resulting from varying degrees of fusion of the predentary. The face of the ventral process is covered in a weakly undulating texture continuing down from the body (Figure 48.2).

Dentary. The large dentary runs from the coronoid process of the mandible to the front of the mouth (Figures 46, 49) and most closely resembles that of Tenontosaurus dossi. There are two pronounced grooves running along the element: the dorsal 


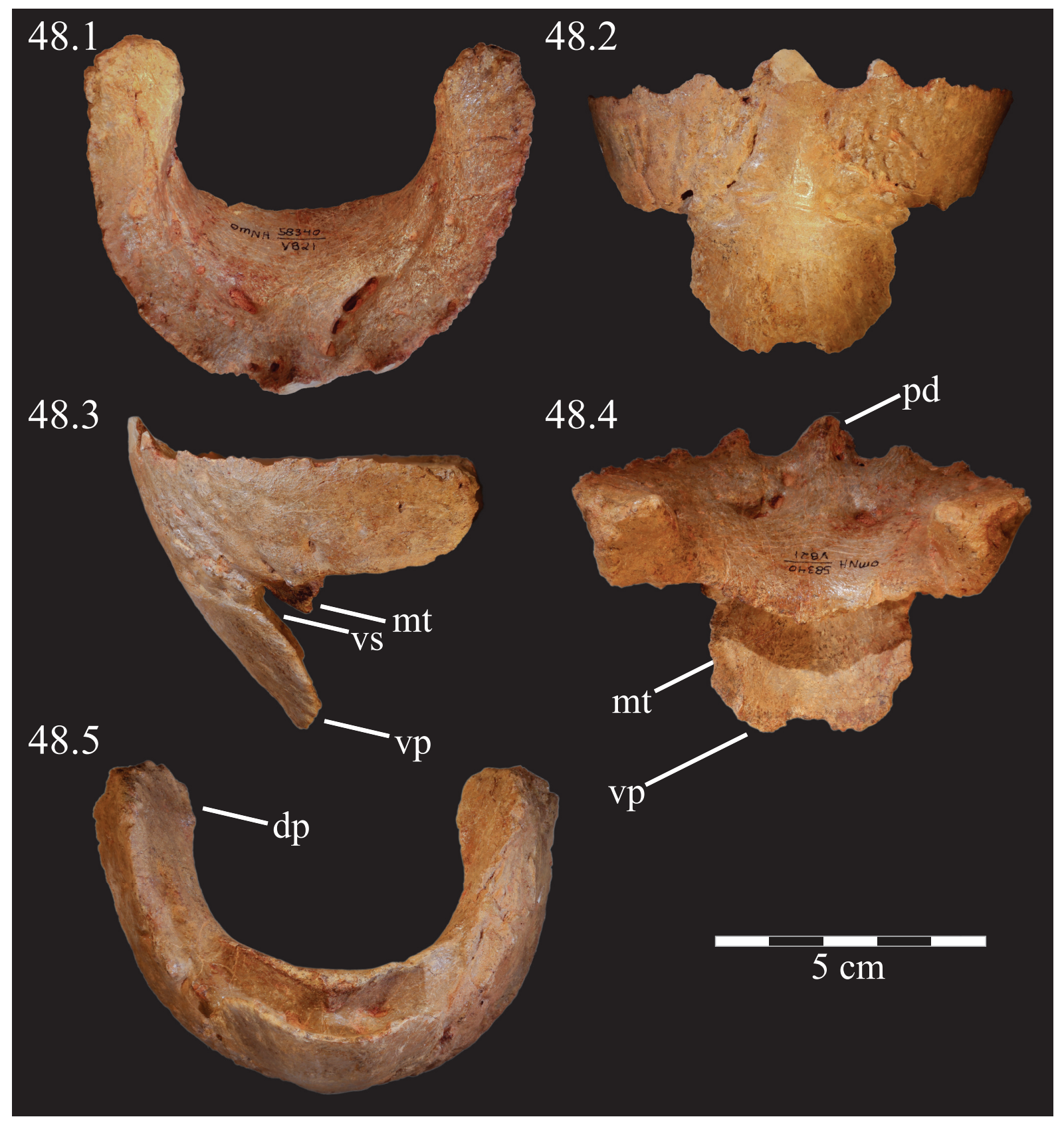

FIGURE 48. Photographs of the predentary of OMNH 58340 in dorsal (48.1), anterior (48.2), lateral (48.3), posterior (48.4), and ventral (48.5) views. Abbreviations: dp - dentary process; mt - median tab; pd - primary denticle; vp - ventral process; vs - ventral sulcus.

alveolar trench (Figure 49.1) and the medial Meckelian canal, which tapers to end just below the anteriormost tooth (Figures 47, 49.3). In articulation, the alveolar trench runs in an anterolaterally concave arch, with the tooth row oriented axially in front and curving out behind.

Although slightly taller behind, the dentary is rectangular in medial aspect (Character 54:1) with two main deviations: the posterodorsally-angled coronoid process and the tapering mandibular symphysis (Figure 49.3). The dentaries curve ventromedially toward the symphysis in the spout-like fashion characteristic of ornithopods and articulate there with the predentary above (Figures 49.1, 49.3). There are grooves on the medial dentary near the symphysis, perhaps for the attachment of a cartilaginous pad, although this area is not well preserved on either side. There are also several 


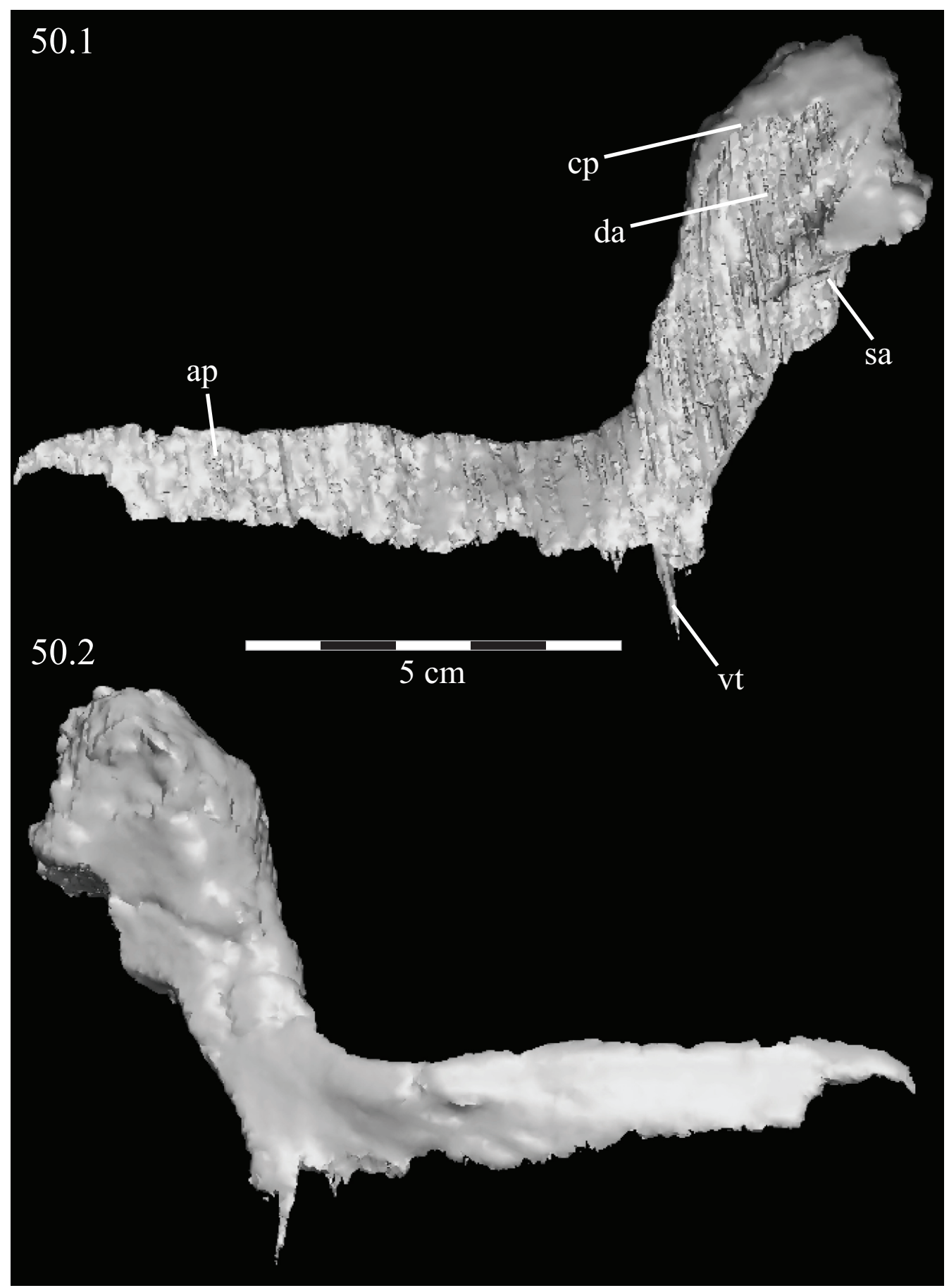

FIGURE 49. Dorsal (49.1), lateral (49.2), and medial (49.3) views of the virtual left dentary of OMNH 58340, disarticulated. Anterior is to the left in the first two images and to the right in the third. Abbreviations: at - alveolar trench; au - alveolar undulations; ca - area for articulation with coronoid; Mc - Meckelian canal; pf - predentary foramina; sa area for articulation with surangular.

small foramina here that probably communicated neurovasculature to the corresponding foramina in the ventral sulcus of the predentary.

One or two of these dorsal foramina communicate with a small canal that runs nearly the entire length of the dentary, parallel to and just above the Meckelian canal (Figure 49.3). Norman (1980) described a similar feature in Iguanodon bernissartensis. A series of large dental foramina communicate with this canal from the lateral surface of the 
element (Figures 46, 49.2). These likely transmitted vasculature and branches of the mandibular ramus of the trigeminal nerve $(\mathrm{CN} \mathrm{V})$ to the cheeks, as in most ornithopods (e.g., Hypsilophodon foxii, Corythosaurus casuarius). Posteriorly, the dentary overlays the surangular above and the angular below (Figure 46).

The large dentary foramina line up along the buccal ridge, which leads to the coronoid process behind (Figures 49.1, 49.2). A narrow shelf of the dentary creates a small pocket between this process and the teeth (Character 59:0). Although smaller, the shelf is reminiscent of that found in ornithopods more derived than Iguanodon bernissartensis (Figure 7, e.g., Ouranosaurus nigeriensis, Edmontosaurus regalis). The coronoid process of the dentary fits into the larger of two furrows on the outside of the coronoid bone (Figures 49.2, 50) but does not reach the apex of the overall process (Figure 46), contra Ostrom (1970). The dorsal angle of the surangular fits into a furrow on the outside of the dentary (Figures 46, 49.2), similar to one described by Galton (1983) for Dryosaurus altus. Finally, the coronoid process of the dentary forms part of the roof of the adductor fossa below, into which $M$. adductor mandibulae inserted (Ostrom, 1961).

The adductor fossa and Meckelian canal both lie in the lower medial surface of the dentary (Figures $47,49.3)$. The medial side of the canal is walled by the splenial, which overlaps the dentary above the canal and abuts the element below it (Figure 47). The anterior process of the prearticular overlaps part of the medial wall of the dentary (Figures 47,51 ).

A shallow, ventrally arched sulcus, regularly perforated with a series of foramina, runs above the canal on the medial side of the dentary (Figure 47 ), along the entire tooth row, mirroring a similar feature found on the maxilla (see description of the maxilla above). The alveolar parapet lies above the sulcus and is covered in the same fine ornamentation as that found on the maxilla (Figure 23). The parapet is partially overlapped behind by the anterior process of the coronoid until the sixth tooth locus (Figures 47, 49.3). The dentary fits closely to the erupted teeth (Figure 49.1). Within the alveolar trench, the alveoli create an undulating pattern along their dorsal surface (Figure 49.1). There are 14 tooth families present in both dentaries of OMNH 58340 (Figure 52, Character 71:14). One large posterior tooth perforates the Meckelian canal.
Coronoid. The coronoid of the mandible is Lshaped in medial aspect, with a wide coronoid and a narrow anterior process (Figure 50, Characters 57:0, 65:0). This corresponds well with Hypsilophodon foxii (Galton, 1974a, figure 10). In more derived iguanodontians (Figure 7, e.g., Mantellisaurus atherfieldensis, Iguanodon bernissartensis), the coronoid diminishes to occupy a small space on the inside of the coronoid process of the dentary, and the bone is lost altogether in hadrosaurs (e.g., Edmontosaurus regalis, Corythosaurus casuarius). The paratype specimen, YPM 5456 (Ostrom, 1970, plates 16, 17), appears to be missing most of the coronoid.

The anterior process is medially convex, with straight dorsal and ventral borders that converge to a point in front (Figure 50). Medially, the anterior process borders the final three teeth and part of the alveolar parapet (Figure 47). The process is overlapped on its lower edge by a small piece of the dorsal process of the splenial and overlaps the dorsal process of the prearticular just behind this. There appears to be a small ventral tab here at the junction of the two processes (Figures 47, 50).

The coronoid process of the coronoid is angled posterodorsally and visible in lateral aspect (Figure 47, Characters 56:0, 66:1). The process begins narrowly below but soon swells to become bulbous (Appendix 4: Coronoid). On the outside of the coronoid process, there is a furrow running up along the anterior angle of the bone and a second furrow branching off and heading nearly straight behind (Figure 50.1). The coronoid process of the dentary fills the larger furrow, while the dorsal angle of the surangular occupies the second (Figure 46). The posterior border of the coronoid process forms part of the medial wall of the Meckelian canal below. The bulbous tips of the coronoid processes are poorly ossified on both sides, with the cancellous bone exposed. This is likely due to preservation, as the smaller, juvenile specimen, OMNH 2531, retains both processes in much better condition. The coronoid process probably served as the insertion of $M$. pseudotemporalis (Ostrom, 1961).

Splenial. The splenial is a long, narrow splint of bone with a tall dorsal process near its posterior end (Figures 47,53 ). This process causes the element to more closely resemble that of Ouranosaurus nigeriensis (Taquet, 1976) than Hypsilophodon foxii, which extends dorsally as well, although not by means of a process. The borders of the splenials of Mantellisaurus atherfieldensis and Iguanodon bernissartensis appear parallel. 


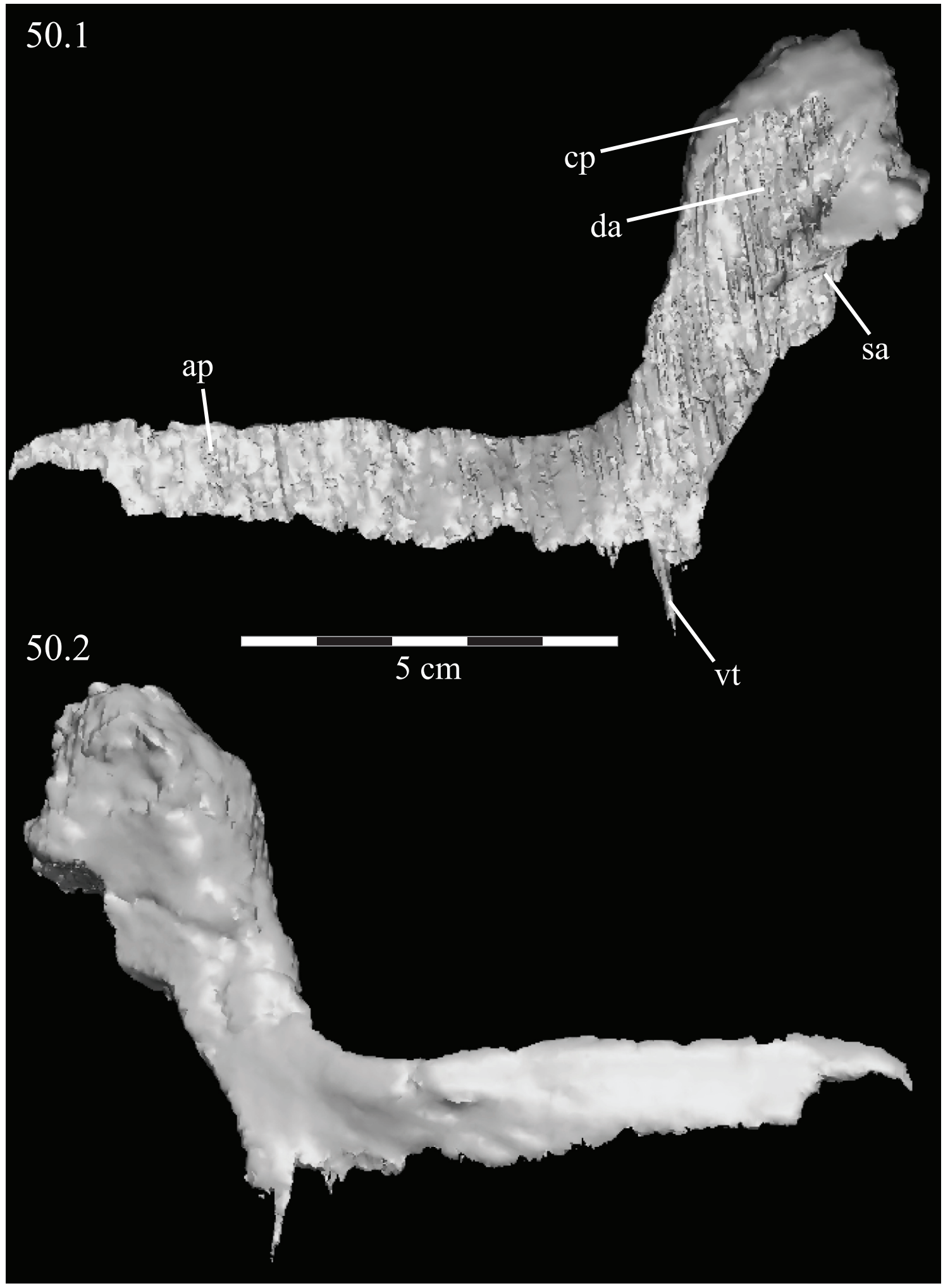

FIGURE 50. Lateral (50.1) and medial (50.2) views of the disarticulated virtual left coronoid of OMNH 58340. The parallel texturing covering most of 50.1 is an artifact of the sectioning process used to isolate the element and represents the articular surface for the surangular and dentary. Anterior is left in 50.1 and right in 50.2. Abbreviations: ap - anterior process; cp - coronoid process; da - articular surface for dentary; sa - articular surface for surangular; vt ventral tab. 


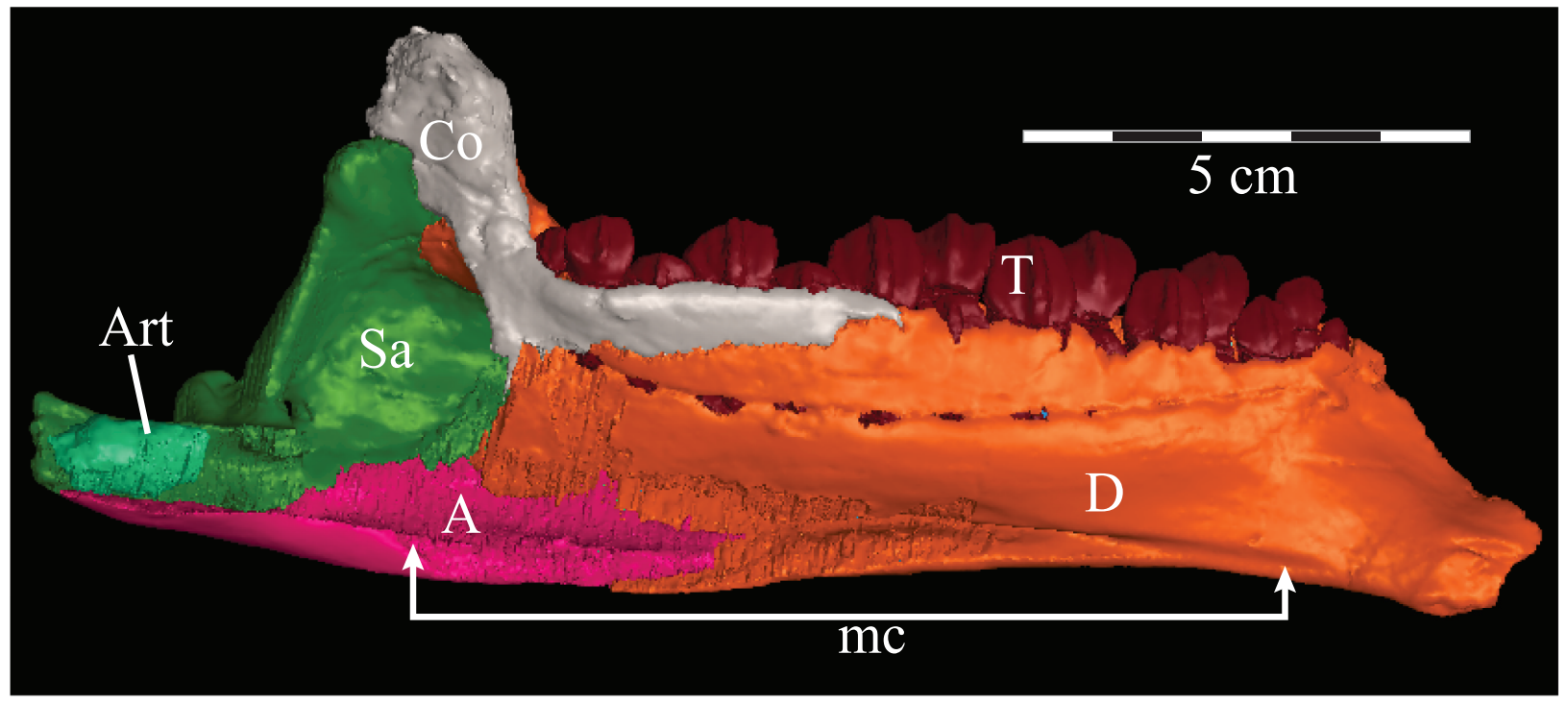

FIGURE 51. Medial view of the virtual left mandible of OMNH 58340 with the splenial and prearticular removed to show the extent of the Meckelian canal as it traverses the mandible. Anterior is right. Abbreviation: mc - Meckelian canal.

The splenial is convex medially and the dorsal process is angled slightly back. The lower edge of the element is rounded medially. There is a ridge running the entire lateral length of the element (Figure 53). Above this ridge is a small furrow, bordered above by a second, posterior ridge running from the posterior tip of the element to the large internal mandibular foramen below the dorsal process (Figure 53, Appendix 4: Splenial). The angular contacts both this furrow and the longer ridge and borders the posterior half of the splenial until it is replaced in front by the dentary. The splenial forms over half of the medial wall of the Meckelian canal forward of the internal mandibular foramen (Figures 47, 51, Character 67:0). The splenial contributes to the canal similarly in Hypsilophodon foxii, Mantellisaurus atherfieldensis, and Iguanodon bernissartensis. In hadrosaurs the splenial is restricted to the rear of the canal (e.g., Edmontosaurus regalis, Corythosaurus casuarius). The splenial forms all but the posterodorsal corner of the border of the internal mandibular foramen, which is formed by the prearticular (Figure 47).

The dorsal process of the splenial lies against the dentary medially and its upper tip covers part of the anterior process of the coronoid (Figure 47). Forward of the process, the element gently tapers and the anterior tip splits into dorsal and ventral points. These may be due to preservation or preparation; it is possible that the element terminated more regularly. The splenial tapers to end in a point behind the process and foramen.
Angular. The angular is a simple bone, flat below and arched above, that terminates in a point at both ends (Figure 54). The angular is nearly half the length of the mandible, although it is only visible along the posterior third (Character 63:0) as it floors the retroarticular process (Figure 46). The angular is similarly visible in lateral aspect in basal ornithopods and most basal iguanodontians (e.g., Hypsilophodon foxii, Ouranosaurus nigeriensis). However, in Mantellisaurus atherfieldensis, Iguanodon bernissartensis, and some other more derived iguanodontians (Figure 7, Appendix 3), the element is lower and less visible. In hadrosaurs, the element is only visible medially (e.g., Edmontosaurus regalis, Corythosaurus casuarius).

There is a ridge on the medial surface of the angular running along the rear third of the element (Figure 54.2). Another ridge begins below the former and extends the rest of the way forward. Above both ridges is a furrow running the entire length of the bone. The lateral surface of the angular is smooth except for a very shallow fossa running under the tallest part of the bone.

The angular overlaps the surangular for most of its length until the surangular shifts laterally to lie in the furrow mentioned above (Figures 46, 54.2, 55.1 , Character 64:0). In ornithopods basal to Mantellisaurus atherfieldensis (Figure 7), the lateral visibility of the angular appears to be related to its overlapping the surangular. When the angular becomes less visible in more derived taxa, it tends to insert in a ventral groove in the surangular. 


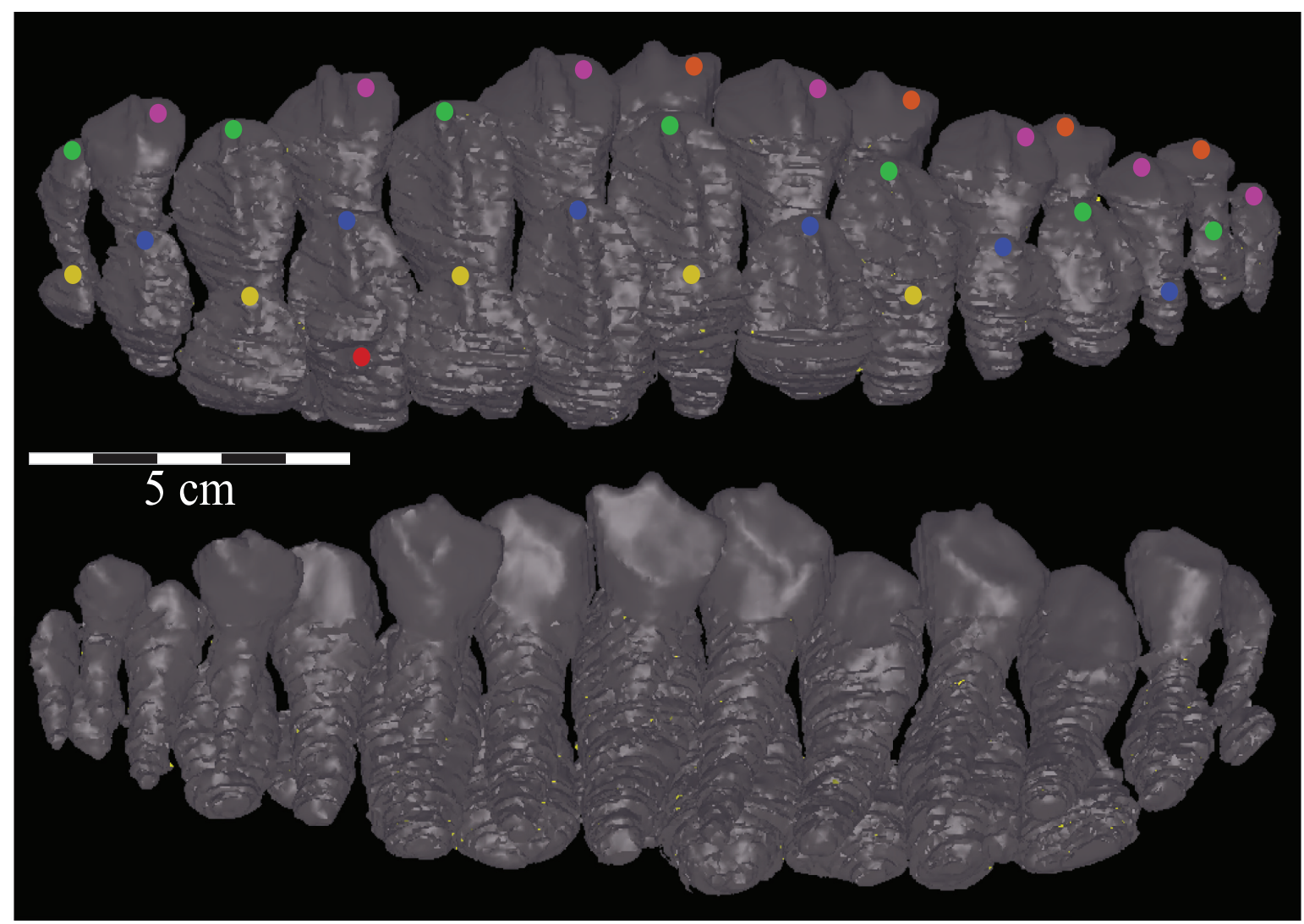

FIGURE 52. Lingual (above) and labial (below) views of the complete virtual tooth set of the left dentary of OMNH 58340 , demonstrating the method of replacement, with groups of teeth beginning to be replaced in alternating succession from posterior to anterior. Individual Zahnreihen are indicated by dots of the same color placed at the occlusal tips of the teeth. Anterior is to the left in the upper image and to the right in the lower image.

When, as in hadrosaurs, the element becomes visible only medially, the angular simply abuts the surangular.

The angular articulates with the prearticular medially along most of its length (Figures 54.2, 55.1). The articular contacts the posterior tip of the element (Figure 51). The dentary overlaps the front third of the angular and, there and behind, the angular forms the lower part of the outer wall of the Meckelian canal (Figure 51). The angular meets the splenial medially, with the back half of the latter fitting between the longer ventral ridge and the lower edge of the former.

Surangular. There are two main components to the surangular: an anterior wing and a retroarticular process (Figures 46, 55). The element is transversely thin and slightly convex laterally.

The anterior wing is triangular in lateral aspect with anterior and dorsal angles, and a ventral angle that contributes to the retroarticular process. Thick medial ridges run from the dorsal angle to the other two angles (Figure 55.2). There is a medially oriented boss on the dorsal angle that probably served as the insertion of $M$. pseudotemporalis (Ostrom, 1961). The M. adductor mandibulae externus group likely inserted between the boss and the retroarticular process (Ostrom, 1961).

The ventral angle is perforated by the surangular foramen (Character 69), which probably carried the cutaneous branches of the inferior alveolar nerve, as in modern lizards (Oelrich, 1956; Galton, 1974a). A similar foramen is found in nearly all non-hadrosaurid ornithopods (e.g., Hypsilophodon foxii, Ouranosaurus nigeriensis). There is a lateral boss behind the foramen (Figures 56,57 ), which is present in ornithopods basal to Mantellisaurus atherfieldensis (Figure 7, e.g., H. foxii, Thescelosaurus neglectus, Tenontosaurus dossi), but becomes an everted lip in basal iguanodontians including and more derived than M. atherfieldensis (e.g., Iguanodon bernissartensis). Medial to the boss is a groove (Figure 56), which forms part of the mandibular articular cotyle, together with the prearticular.

The surangular projects medially and forms the lateral part of the retroarticular process of the 


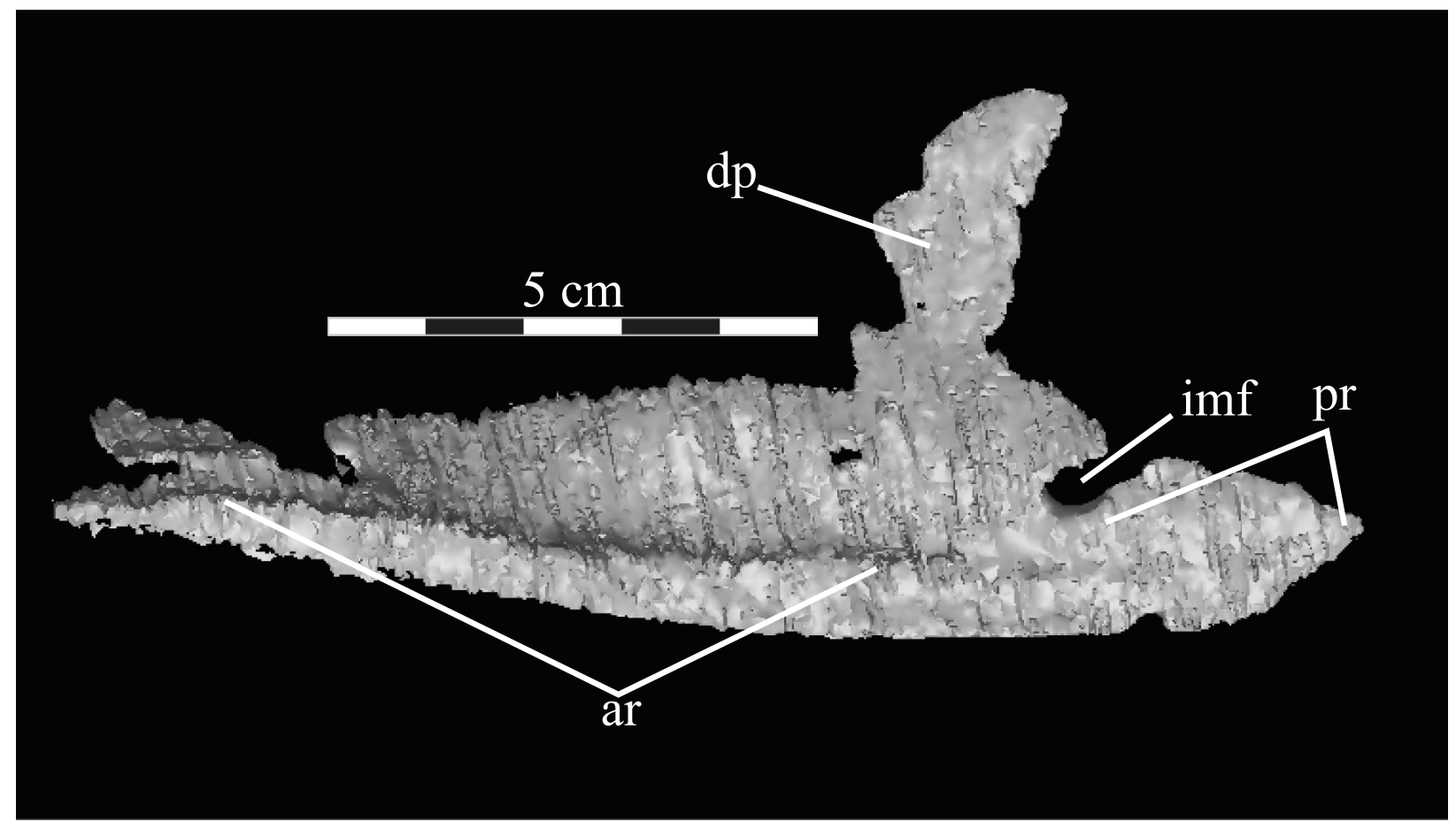

FIGURE 53. Lateral view of the virtual left splenial of OMNH 58340, disarticulated. Anterior is left. Abbreviations: ar axial ridge; dp - dorsal process; imf - internal mandibular foramen; pr - posterior ridge.

mandible (Figures 56, 57). The underside of this projection is arched and forms a short canal with the corresponding lateral process from the prearticular (Figure 58.1), which may have carried retrograde neurovasculature from the Meckelian canal or contained unpreserved portions of the articular. This arch soon recedes and the rear of the medial surface is smooth.

The retroarticular process of the surangular is rectangular in lateral aspect and thicker above, with its ventral border angled inward, and is slightly upturned behind (Figures 46, 57). This dorsally angled retroarticular process is common to most ornithopods and becomes exaggerated in hadrosaurs (e.g., Edmontosaurus regalis, Corythosaurus casuarius). A shallow fossa extends from behind the lateral boss to the posterior end of the element, which Ostrom (1961) identified as the insertion of M. pterygoideus ventralis.

The anterior angle is overlapped by the dentary but extends underneath to the final dental foramen (Figures 46, 57). The dorsal angle fits into the smaller furrow on the outside of the coronoid and meets the coronoid process of the dentary (Figure 47). The surangular forms a scarf joint with the angular below for nearly its entire length; only its anterior and posterior tips are excluded (Figure 59, Character 64:0). The posterior tip of the element just touches the prearticular under the articular (Figure 58.1). The articular lies against the flat medial surface of the retroarticular process of the surangular. The surangular forms a significant portion of the outer wall of the Meckelian canal (Figure 51).

Prearticular. The prearticular is L-shaped in lateral aspect, with anterior and retroarticular processes (Figures 47, 58.2, Character 68:0). The rear of the anterior process is angled forward while its leading edge is nearly vertical.

There is a short waist on the retroarticular process just behind the anterior process. The prearticular then swings up into a prominent saddle-hornshaped projection, behind which are the larger lateral and smaller medial articular fossae (Figure 56, Appendix 4: Prearticular). Here, the prearticular bridges a canal to join the surangular laterally (Figure 58.1). In Hypsilophodon foxii, the element remains narrow along its length and does not meet the surangular in this fashion.

The articular fossae of the prearticular are sharply defined and form, together with the surface of the surangular medial to the ventral boss, the preserved articular cotyle of the mandible for the quadratic condyle. Both mandibles were found with this area in very close association with the quadratic condyles. The mandible does not articulate 


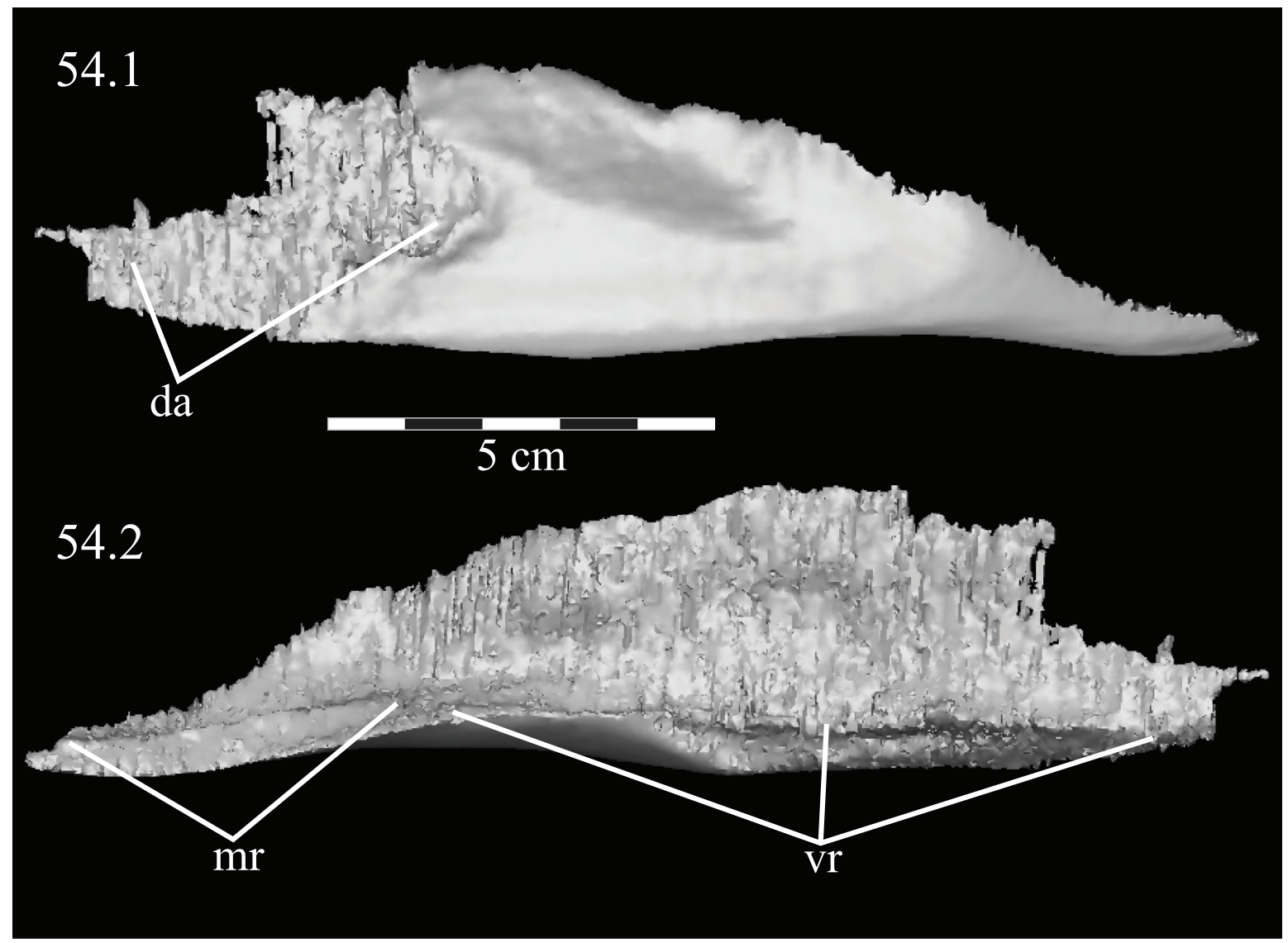

FIGURE 54. Lateral (54.1) and medial (54.2) views of the virtual left angular of OMNH 58340. Anterior is left in the upper image and right in the lower image. Abbreviations: da - articular surface for the dentary; mr - medial ridge; $\mathrm{vr}$ ventral ridge.

properly with the suspensorium unless this surface is used, indicating that the articular is nearly, if not entirely, excluded from articulation with the cranium. In Mantellisaurus atherfieldensis and Iguanodon bernissartensis, the prearticular extends laterally but does not participate in the articular cotyle as it does here. The only other taxon considered in this study in which this element contributes to the articulation with the quadrate is Theiophytalia kerri (Gilmore, 1909).

The retroarticular process of the prearticular is medially convex except for a strong furrow behind, which probably served as the insertion for $M$. depressor mandibulae (Ostrom, 1961). This area is better preserved on the right mandible (Figure 47). The lateral side of the element is correspondingly concave, and the articular fits into a pocket between the prearticular and the surangular (Figure 57).

The ventral surface of the prearticular is broad and flat and abuts the angular along its entire length (Figure 47). As the element swings up to the anterior process in front, the prearticular is overlapped by the splenial (Figures 47, 58.2) and fills the gap left by the latter in the border of the internal mandibular foramen (Figure 47). In Theiophytalia kerri, this foramen is wholly contained within the splenial. Above this, the anterior process is overlapped by the lower corner of the coronoid.

Articular. The articular is a small, roughly cylindrical, poorly ossified bone (Figures 51,57 ). There is very little lamellar bone present. The poor state of preservation is consistent with other ornithopods, making systematic analysis of this element very difficult. The lateral and dorsal surfaces of the element are a little flatter than the other sides. The articular tapers to end flatly in front and in a point behind.

The articular fits in a pocket bounded by the retroarticular processes of the prearticular and surangular on its sides, and a very small piece of the angular below (Figures $47,51,57$ ). It is possible that the articular may have extended forward to articulate with the quadrate. However, participation 


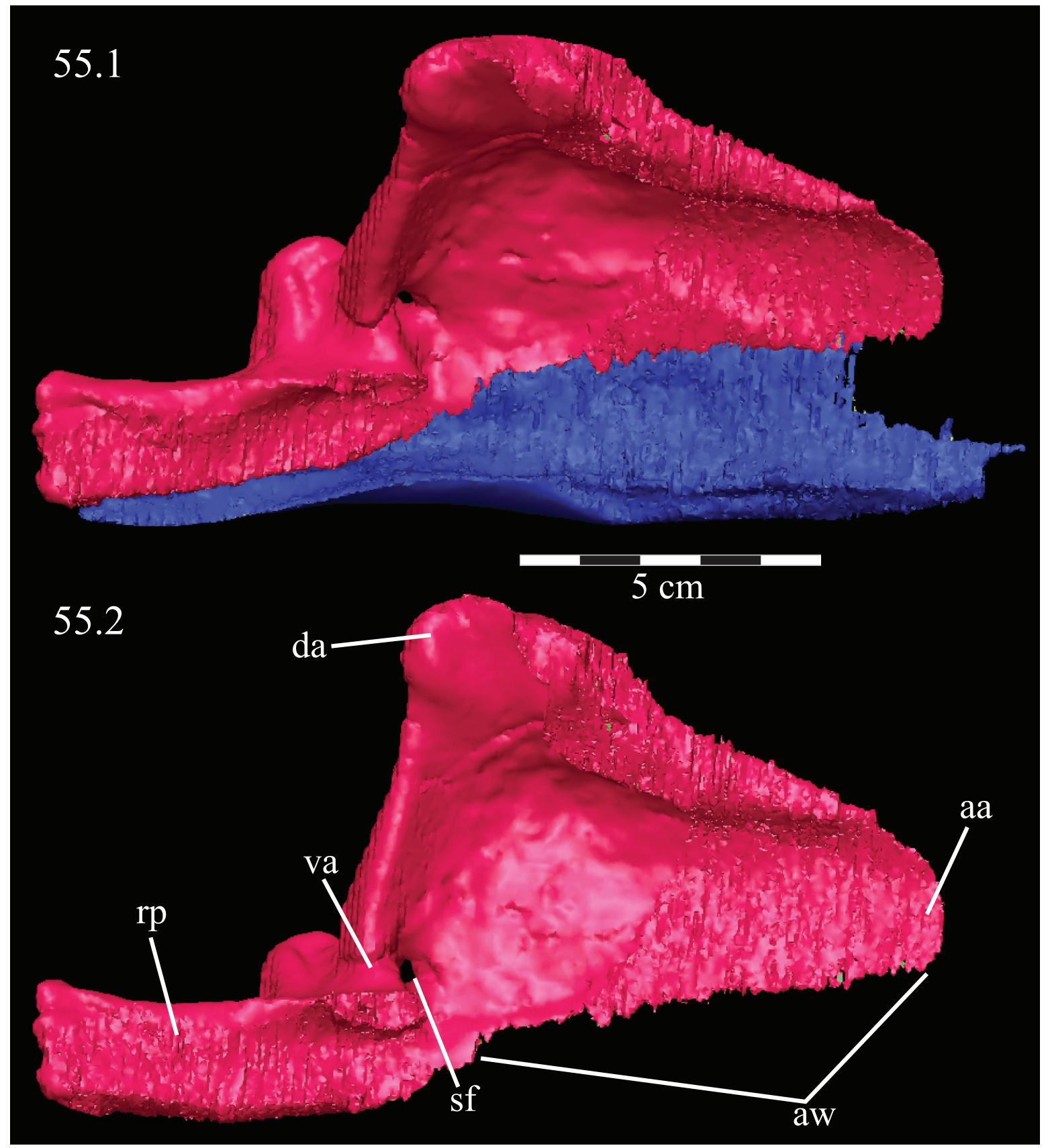

FIGURE 55. Medial view with the dorsal tip rotated lingually (55.1) of the virtual left angular (blue) and surangular (pink) and medial view (55.2) of the disarticulated virtual left surangular of OMNH 58340. Anterior is to the right. Abbreviations: aa - anterior angle; aw - anterior wing; da - dorsal angle; sf - surangular foramen; rp - retroarticular process; va - ventral angle.

in the quadratic cotyle is impossible without this unpreserved extension.

Dentition. The teeth in OMNH 58340 are largely preserved in articulation with the maxilla and dentary, the only notable exception being the majority of the functional tooth row of the right maxilla, which is lost (Figures 3,9). For this description, the common dental directional terminology is used: mesial (toward the articulation of the paired dentaries), distal (toward the mandibular/cranial articulation), buccal (toward the presumed cheeks), lingual (toward the tongue), apical (toward the crown of the tooth), and thecal (toward the tooth root).

In occlusal view, the tooth row is shaped in a buccally concave arch, which is angled such that 


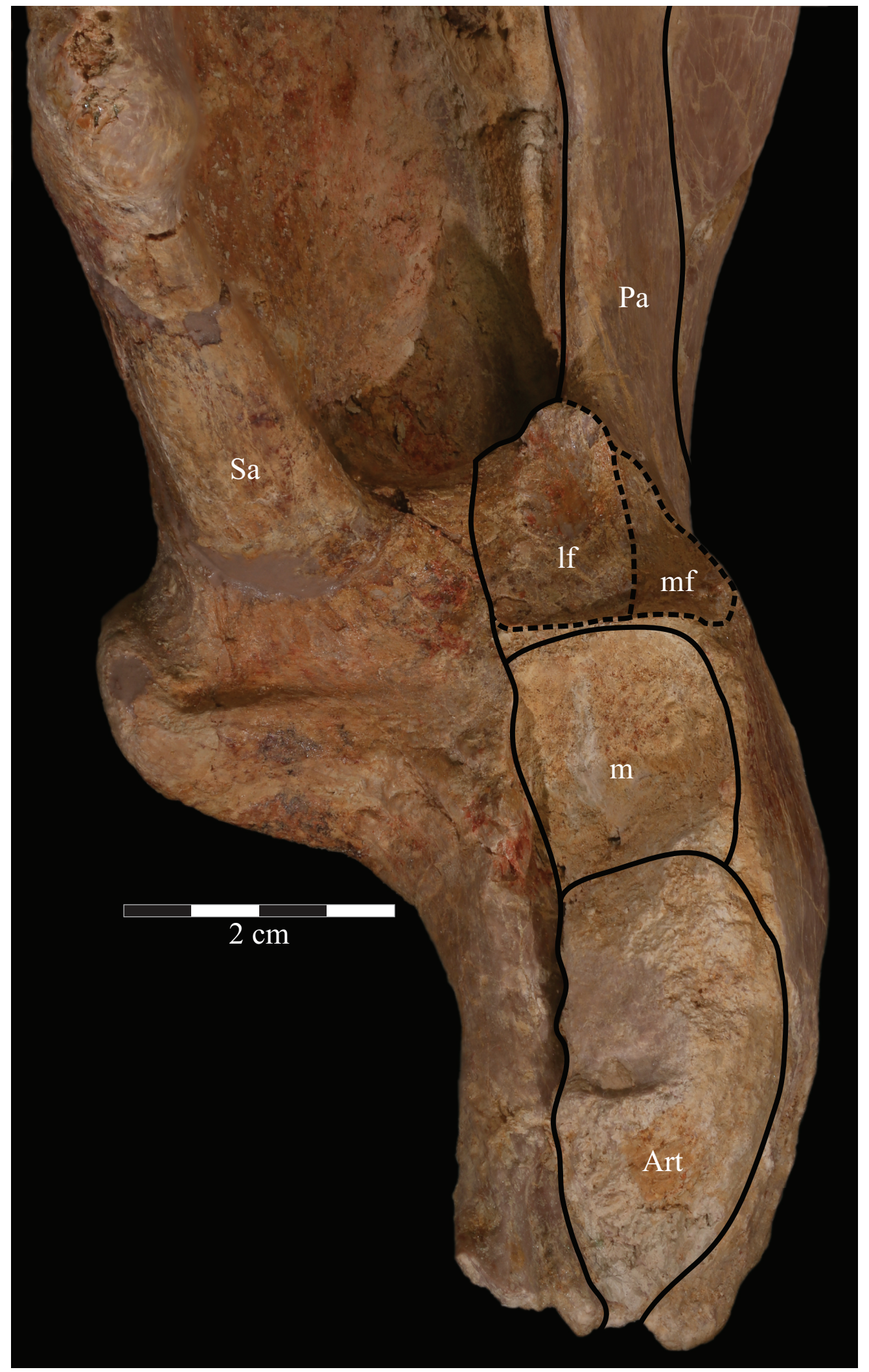

FIGURE 56. Photograph of the articular surface of the left mandible of OMNH 58340, detailing the facets present on the dorsal surfaces of the surangular and prearticular. The dashed lines circumscribe the articular fossae of the prearticular. Anterior is up. Abbreviations: If - lateral articular fossa; $\mathrm{m}$ - matrix; $\mathrm{mf}$ - medial articular fossa. 

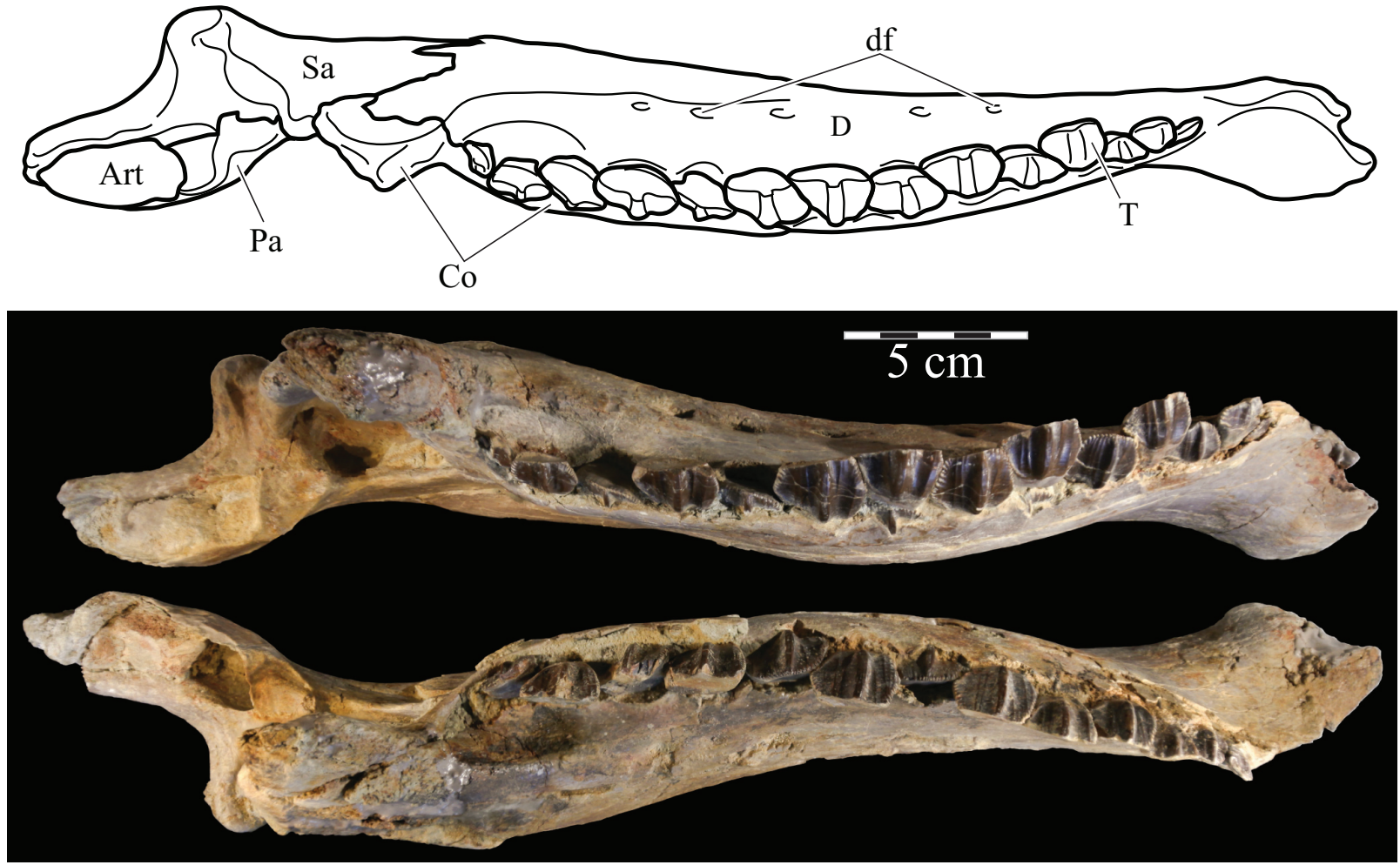

FIGURE 57. Dorsal schematic of the left (above) and photographs of the left (middle) and right (below) mandibles of $\mathrm{OMNH}$ 58340. Anterior is to the right. Abbreviation: df - dental foramina.

the tooth row runs in a straight line behind the beak and bows outward distally (Figures 6, 57). Teeth are largest in the middle of each tooth row, becoming smaller mesially and distally (Figure 52). Both the maxillary and dentary teeth are arranged en echelon, with the distal edges of each tooth lingually overlapping the tooth behind it. The teeth are not unilaterally enameled (Figure 60), as suggested by Ostrom (1970).

In the maxillary tooth rows, there are a total of 13 vertical tooth rows, or families, each with a maximum of two teeth present (Character 30:13). There are 14 tooth families in the dentaries (Character 71:14), with three teeth in four of the rows (three on the left and one on the right) and two in all of the rest. The presence of a second replacement crown significantly widens the phylogenetic distribution of this characteristic, previously thought to occur only in ornithopods more derived than Iguanodon bernissartensis (Figure 7, e.g., Probactrosaurus gobiensis, Edmontosaurus regalis). There are not multiple teeth occluding in a single family, as is the case in certain iguanodontians more derived than I. bernissartensis (Figure 7, e.g., P. gobiensis) and all hadrosaurs (e.g., Corythosaurus casuarius).
In general form, the teeth of Tenontosaurus tilletti most closely resemble those of ornithopods basal to Iguanodon bernissartensis and Mantellisaurus atherfieldensis (Figure 7), including Hypsilophodon foxii, Theiophytalia kerri, and Dryosaurus altus. These taxa share spatulate teeth completely coated in enamel, a prominent carina on the lingual surface of the dentary teeth, and no prominent carina on the buccal surface of the maxillary teeth, which instead bear several small, subparallel grooves and ridges with a cingulum along the base.

Each unworn maxillary tooth in Tenontosaurus tilletti is roughly elliptical in lingual profile, with a prominent distal apical corner (Figure 23.1). Each tooth is lingually concave, with root and unworn crown subequal in length (Figure 60). A pronounced cingulum runs along the buccal base of each crown and, on the same surface, a faint median and several smaller ridges run apically (Figures 23.1,60). The effect of the cingulum is to make the face of each tooth appear recessed. Along the mesial and distal margins of each tooth, running from the base of the crown to the apex, is a series of enameled denticles, which decrease in size nearer the crown (Figure 23.1). Within the maxilla, each developing tooth is solely composed 


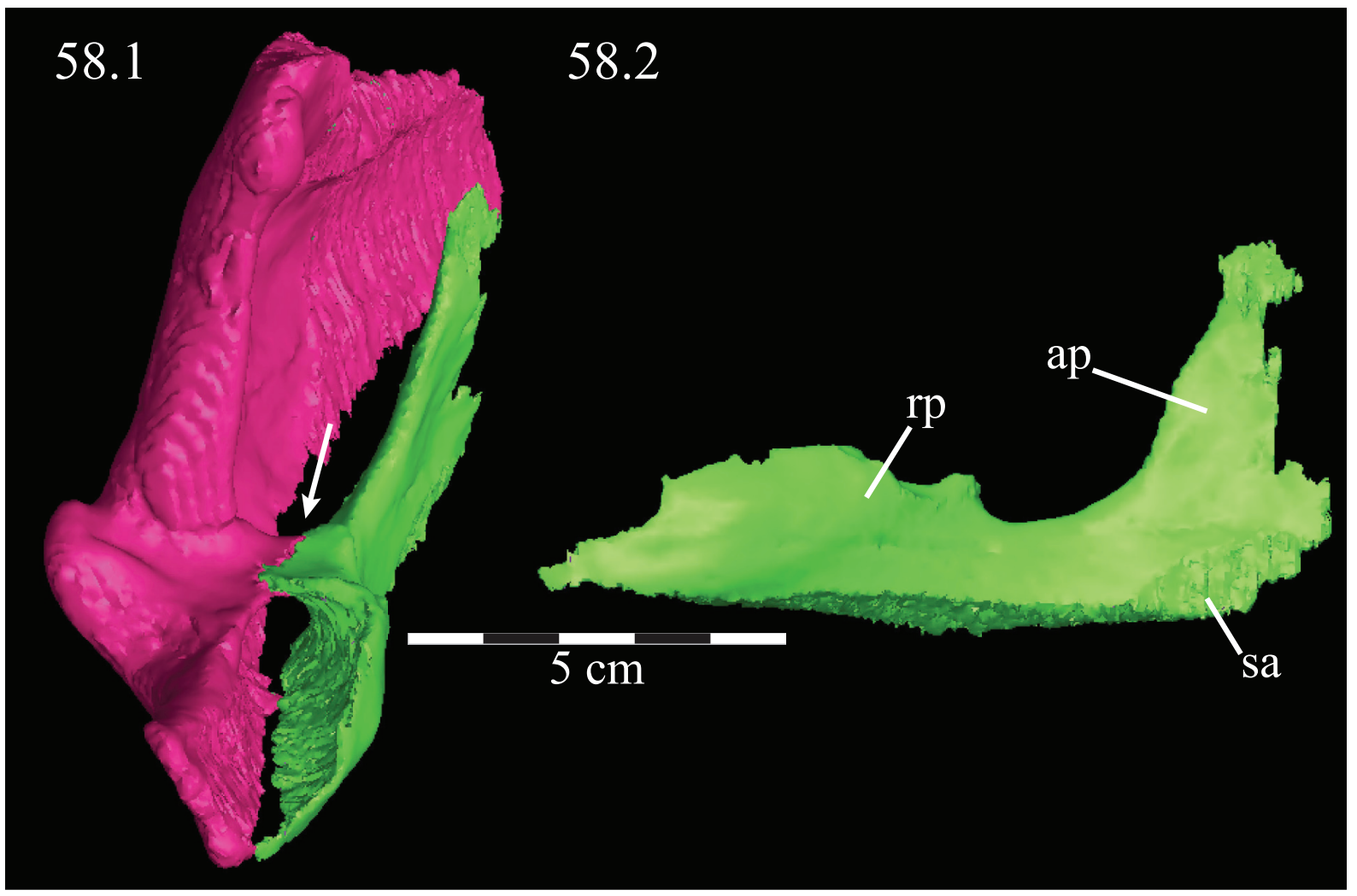

FIGURE 58. Posterodorsal view (58.1) of the virtual left surangular (pink) and prearticular (green) and medial view (58.2) of the virtual left prearticular of OMNH 58340. The left image shows the nature of the articulation of the two elements at the point of articulation of the mandible with the quadrate (indicated by arrow). Anterior is into the page in 58.1 and to the right in 58.2. Abbreviations: ap - anterior process; rp - retroarticular process; sa - area of articulation with the splenial.

of the crown (Figure 60). Wear begins mesiolingually on newly erupted teeth.

The dentary teeth are generally similar to those of the maxilla, save that they are concave buccally, with their thickly enameled crowns oriented lingually (Figures 23.2, 60). A single dentary tooth pierces the roof of the left Meckelian canal. The lingual surface of the dentary teeth bears a mesially offset, prominent carina running from the base of the crown to the apex, which is surrounded by a few smaller ridges on either side, usually one to two mesially and two to three distally (Figure 23.2, Character 70:1). This is in contrast with most other basal iguanodontians (e.g., Theiophytalia kerri), in which the primary carina is distally offset. The secondary ridges tend to be oblique to the long axis of the teeth more so than the primary ones. The dentary teeth have a more angular outline than the maxillary teeth, but they bear similar marginal denticles (Figure 23).

The occlusal surface of each tooth is offset at an approximately 45-degree angle from the long axis of the crown. The wear on the teeth is uneven, and is apparently concentrated, or at least begun, on the distal end in dentary and the mesial end in maxillary teeth. This results in dentary teeth with taller mesial occlusal corners and maxillary teeth with taller distal ones, and produces a 'stair step' effect along the occlusal edge (Figures 23.2, 52). Because of an uneven distribution of enamel and dentine in the tooth, the occlusal surface of each tooth is slightly concave, with a high point at the leading occlusal edge and an increasingly deep fossa down along the occlusal surface. There are no secondary wear surfaces present on the teeth.

All teeth are replaced lingually (Figure 52) in a similar pattern (Edmund, 1960). There are waves of teeth, or Zahnreihen, which begin to erupt distally, alternating alveoli. There are six Zahnreihen in the left dentary, with a z-spacing of 1.40 , and four in the left maxilla with a z-spacing of 1.44 . These z-spacings fall outside the range (1.75-2.5) generally accepted for reptiles (Osborn, 1975). Further analysis into this feature throughout Ornithopoda must be carried out before meaningful comparison can be made. The roots of older, 

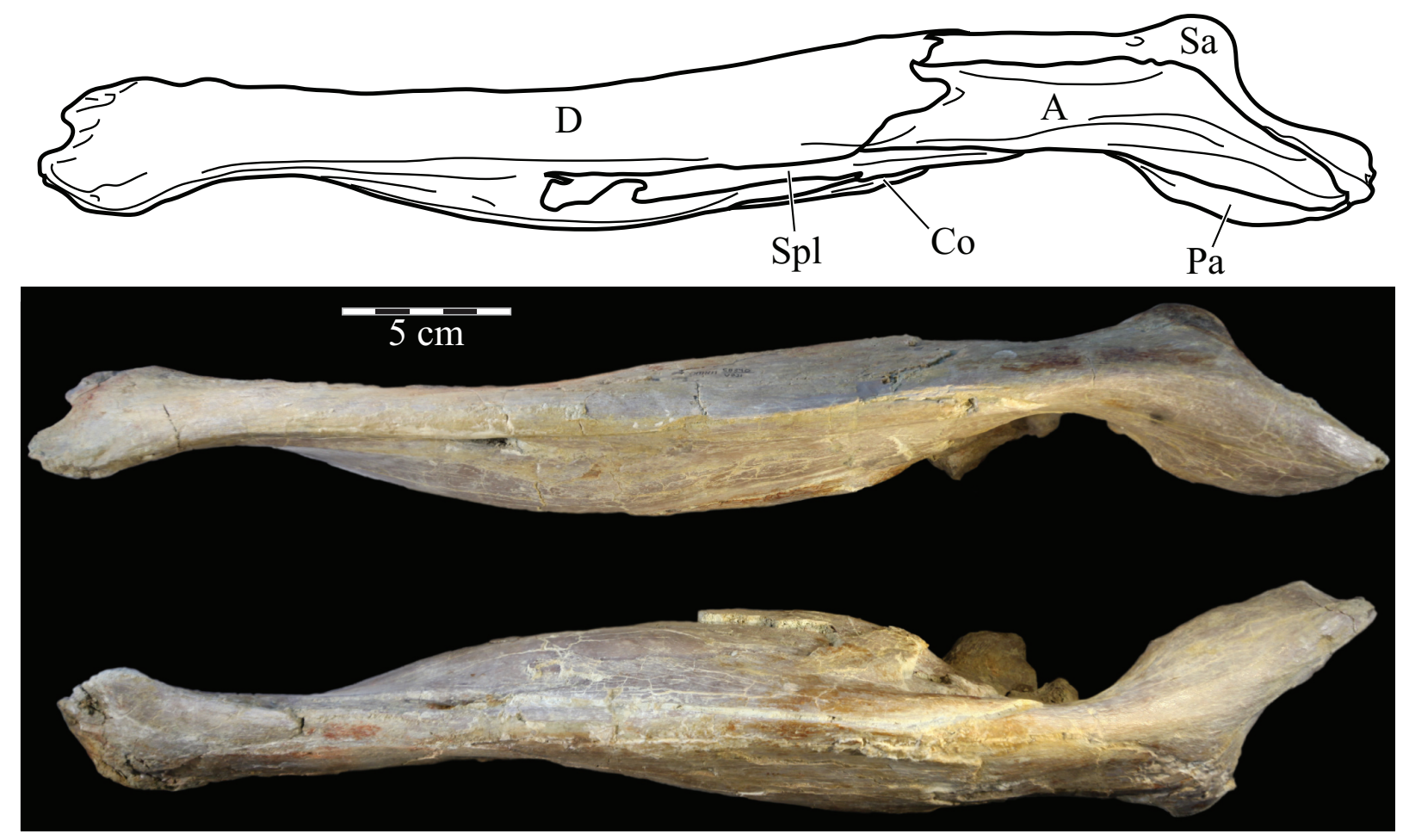

FIGURE 59. Ventral schematic (above) and photographs of the right (middle) and left (below) mandibles of OMNH 58340. Anterior is to the left.

erupted teeth are apparently resorbed as the new teeth develop and move towards eruption, resulting in the crowns of new teeth being partially contained within the roots of old ones (Figure 60).

\section{Accessory Elements}

These elements do not directly articulate with any of the others in the skull and were found displaced from their original positions.

Hyoid Apparatus. Both of the first hyobranchials (Figure 61) of OMNH 58340 were preserved between the mandibles, in close association with the rest of the skull. The poorly ossified anterior end of each bone, which likely articulated with the hyoid itself via a cap of hyaline cartilage, is expanded into a pronounced block. The block quickly tapers behind and the hyobranchial ends in a blunt, rounded tip. Each element is curved to create a ventrally convex bow. The hyobranchials of Tenontosaurus tilletti most closely resemble those of Dryosaurus altus and Theiophytalia kerri. The elements in Ouranosaurus nigeriensis are dramatically curved, and those of Corythosaurus casuarius continue to be thickened too far behind.

Sclerotic Ring. Within the bottom of the left orbit of OMNH 58340, resting above the ectopterygoid, maxilla, and palatine, is a series of small plates (not illustrated, but visible in CT data) embedded in matrix, which are here interpreted as the remnants of the left sclerotic ring. A few of the plates appear to mirror each other, with an inner and outer counterpart. Most are simply partially overlapped by the following plate. The preservation is too poor, and there are too few plates to provide much information beyond evidence of the existence of the structure in Tenontosaurus tilletti (Figure 2).

\section{SYSTEMATIC ANALYSIS}

\section{Methods}

The nineteen taxa listed for comparison in the introduction were utilized as ingroup taxa for phylogenetic analysis. Lesothosaurus diagnosticus was selected as the outgroup, following common practice for analyses of derived Ornithopoda (e.g., Norman, 2004). A complete list of taxa, with the references used to compile the data matrix, is presented below (Appendix 1).

The full matrix (Appendix 3 ) consists of twenty taxa (19 ingroup taxa and one outgroup taxon) coded for 101 characters (Appendix 2). Seventyone of the characters are cranial and were devised independently for this analysis, nearly doubling the amount used by Norman (2004). The final thirty 


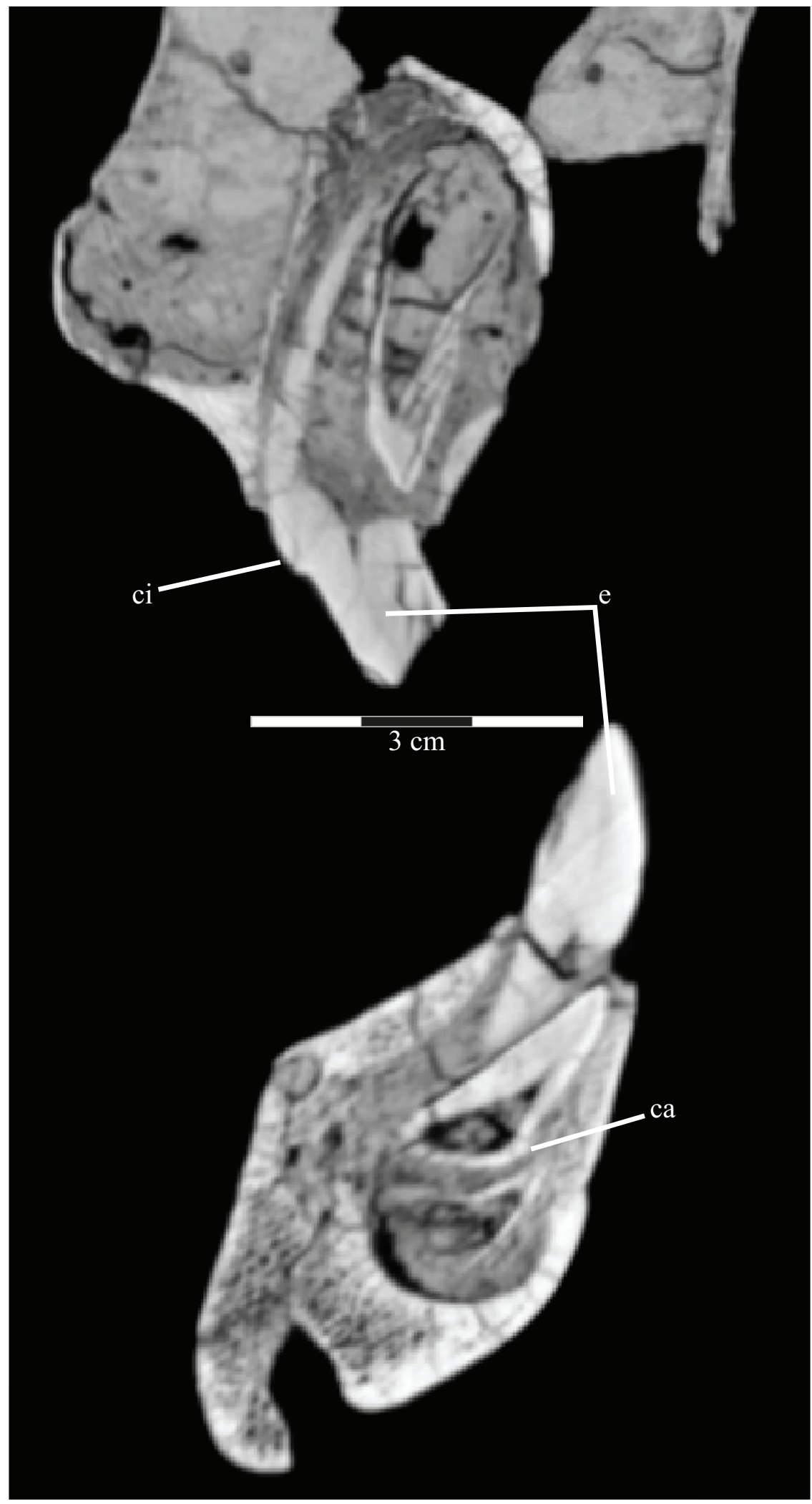

FIGURE 60. Coronal CT images of the maxillary (above) and dentary (below) teeth, taken midway along the length of the respective elements, of OMNH 58340 showing the method of lingual replacement and the concurrent resorption of the tooth being replaced. Dorsal is up and anterior is into the page. Abbreviations: ca - carina; ci - cingulum; e enamel. 


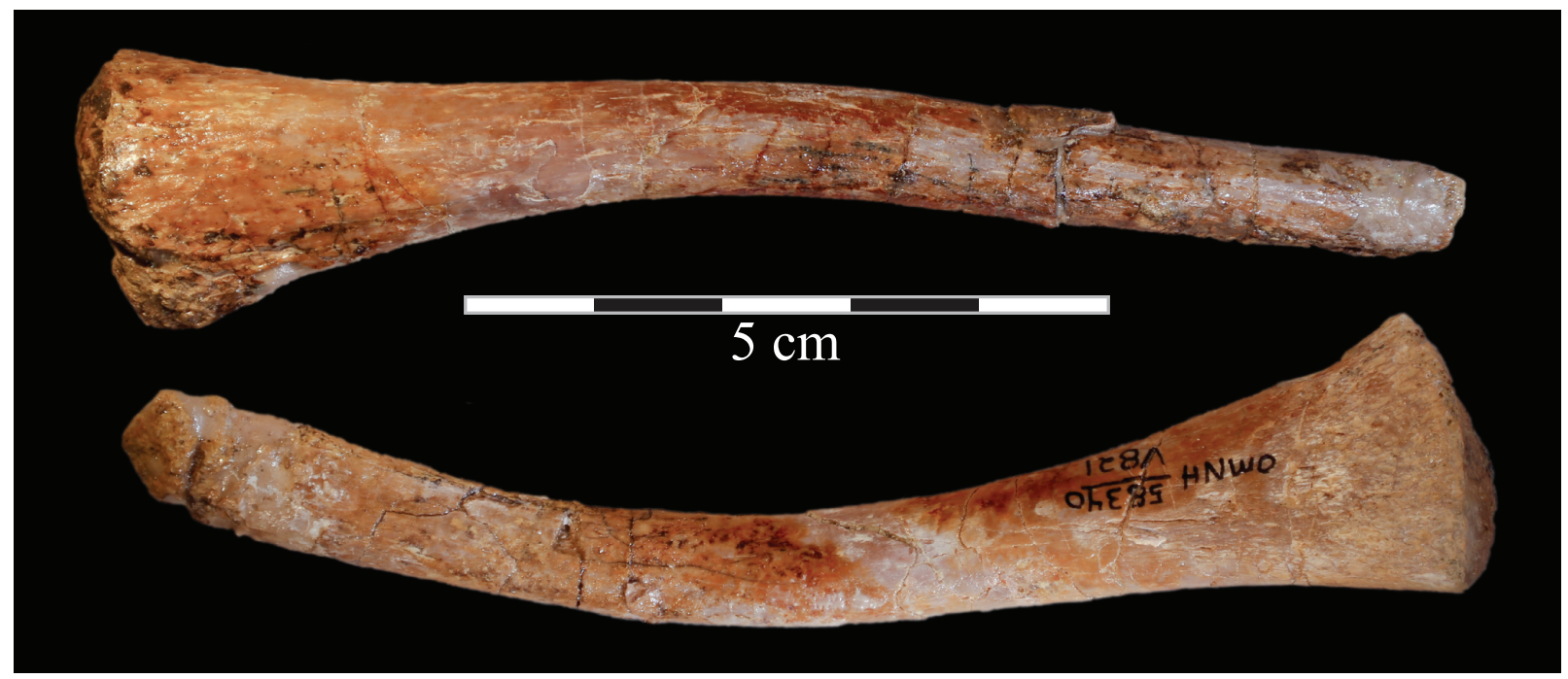

FIGURE 61. Photograph of the left (above, in lingual view) and right (below, in buccal view) hyoids of OMNH 58340. Anterior is to the left in the upper image and to the right in the lower image.

postcranial characters were taken from that study with few modifications. Character similarities shared with other analyses were determined post hoc and are noted below (Appendix 2). Safe taxonomic reduction was found to be unnecessary. The matrix does not contain any taxonomic equivalents and all taxa were included in subsequent analyses.

Analyses were carried out in PAUP 4.0b10 (Swofford, 2003); all characters were treated as unordered and equally weighted, and polymorphisms were treated as uncertainty. The analysis recovered eighteen trees with a minimum number of 277 steps (Consistency Index $(\mathrm{Cl})=0.556$, Retention Index $(\mathrm{RI})=0.696$, Rescaled Consistency Index $(R C)=0.387)$. The strict component consensus (SCC) tree (Figure 7) was well resolved and did not warrant the production of $50 \%$ majorityrule component consensus or Adams consensus trees. The analysis was checked and supported by TNT (Goloboff et al., 2008) and a most parsimonious tree (MPT) was selected to denote unambiguous character transformations (Figure 62).

A Bremer analysis (Bremer, 1994) was completed in TNT, reaching a maximum support value of six within the analyzed taxa and a value of greater than ten (the maximum number of additional steps selected) separating the outgroup from the analyzed taxa (Figure 7). Four of the nodes within the analysis were recovered with a Bremer support value of two; that is, they are absent from the strict consensus of all trees of 279 steps or more. Stronger support is found within several clades (Figures 7). Bootstrap and Group Present/ Contradicted (GC) analyses (Goloboff et al., 2003) were also carried out using TNT, with one thousand replications. Bootstrap and GC support are relatively weak, with support for most clades falling below $50 \%$, although support for several clades (Figure 7, nodes $A-G$ ) appears quite strong. Bremer, bootstrap, and GC values are shown with the SCC (Figure 7). ACCTRAN/DELTRAN analyses were run.

\section{Results}

The strict component consensus tree (SCC) recovered in this analysis (Figure 7 ) is broadly consistent with those recovered in previous analyses (e.g., Weishampel et al., 2003; Norman, 2004; Butler et al., 2008). Thescelosaurus neglectus is recovered as the most derived of the basal ornithopods, as was previously suggested (Butler et al., 2008), and the closest to Tenontosaurus (Figure 7 , node G). The genus Tenontosaurus is recovered as the sister clade to all other iguanodontians (Figure 7 , node A). The clade Rhabdodontidae is not recovered due to the inclusion in this analysis of only one of the taxa generally recovered in this group (Zalmoxes robustus). Dryosauridae is absent for the same reason. Ankylopollexia is recovered with a relatively strong Bremer support value of four (Figure 7, node B), with the notable addition to that clade of $Z$. robustus. Styracosterna is recovered with the highest Bremer, bootstrap, and GC values in the analysis (Figure 7, node $C$ ). Protohadros byrdi and Probactrosaurus gobiensis are recovered in a poorly supported Hadrosauroidea (Figure 7, node D), which includes Telmatosaurus transsylvanicus with much stronger node 


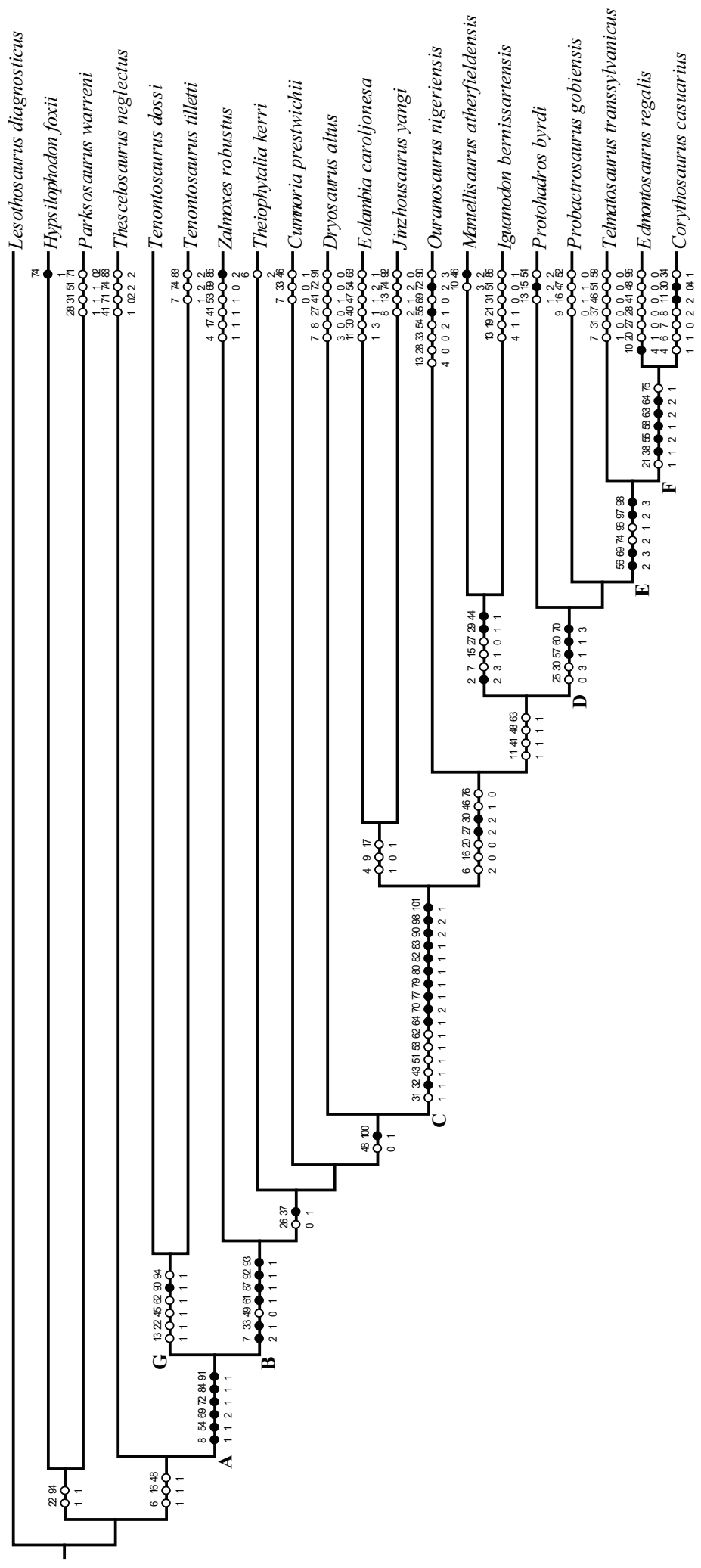

FIGURE 62. Optimized character distribution showing unambiguous character state changes on one of 12 most parsimonious trees (MPTs) recovered in this analysis. Character number and state present are indicated by the number above and below each line, respectively. Synapomorphic character states are indicated by black circles, while white circles denote homoplastic character states. Certain nodes are noted with letters referenced in the text. Optimization was performed in PAUP* version 4.0b10 (Swofford, 2002) and checked in TNT (Goloboff et al., 2008). 
support as the sister taxon to crown Hadrosauridae (Figure 7, node F; sensu Prieto-Márquez, 2010).

All characters and character states discussed in this section are listed in Appendix 2, and a data matrix coding each taxon can be found in Appendix 3.

Iguanodontia. The clade Iguanodontia (Figure 7, node A) is inferred by this analysis to include Tenontosaurus, Corythosaurus casuarius, their most recent common ancestor, and all of its descendants. The clade is supported by six unambiguous synapomorphic characters recovered by the analyses run in both PAUP and TNT (Figure 62, node A, Appendix 3). These include a frontal that contributes less than half of the orbital rim (8:1), parallel dorsoventral borders of the dentary (54:1), a single surangular foramen (69:2), low and square dorsal neural spines (72:1), three phalanges in the third manual digit (84:1), and a pubic shaft shorter than the ischium with no pubic symphysis (91:1).

The following homoplastic characters also help define Iguanodontia. The supraoccipital in this clade (except in Zalmoxes robustus, Dryosaurus altus, Theiophytalia kerri, and Cumnoria prestwichii) is excluded from forming any part of the border of the foramen magnum by the exoccipital (43:1). The relatively high number of basal iguanodontian taxa that do not possess this trait increases the likelihood of convergence between Tenontosaurus and higher iguanodontians in this instance (in all of which the supraoccipital is excluded from the foramen magnum). In addition, the occlusal margin of the predentary is denticulate $(62: 1)$ in iguanodontian taxa with the exceptions of $D$. altus and $Z$. robustus (Appendix 3). The presence or absence of premaxillary teeth is a problematic character, because it splits the genus, Tenontosaurus, which is recovered as the sister taxon to all other iguanodontians. Tenontosaurus dossi retains the plesiomorphic teeth (3:0), while $T$. tilletti does not (3:1). Therefore, either this character cannot be called a synapomorphy of Iguanodontia while allowing Tenontosaurus to remain within the clade, or one of these species must have acquired their respective character state secondarily.

The trait for which Iguanodontia is named, tooth shape, does not code as a synapomorphy for the clade. Rather, there is a spectrum of similar teeth, tending gradually toward the interlocking battery of relatively small teeth in the hadrosaurs (e.g., Edmontosaurus regalis, 30:53, 70:4, 71:50). This trend apparently begins with larger, more spatulate teeth in basal taxa (e.g., Hypsilophodon foxii,
30:11, 70:1, 71:13, Tenontosaurus tilletti, 30:13, $70: 1,71: 14)$ that become progressively smaller and more numerous as the taxa become more derived (e.g., Iguanodon bernissartensis, 30:29. 70:2, 71:25).

Ankylopollexia. The clade Ankylopollexia (Figure 7 , node $B$ ) is inferred by this analysis to include Zalmoxes robustus, Cumnoria prestwichii, Theiophytalia kerri (all unresolved within a polytomy), Corythosaurus casuarius, their most recent common ancestor, and all of its descendants. Ankylopollexia are supported by three unambiguous synapomorphic characters recovered only in the analysis run with TNT, one only recovered in PAUP, and three that were recovered by both analyses. The inclusion of $Z$. robustus and Dryosaurus altus within this clade is unusual (e.g., McDonald, 2012).

The characters uniquely recovered in the analysis performed in TNT were the length of the frontals' suture with the parietal about half the combined width of the frontals $(7: 2)$, the articulation of the jugal with the maxilla displaced anteriorly on the maxilla (33:1), and a bifurcate ventral process of the predentary (61:1). Cumnoria prestwichii and Ouranosaurus nigeriensis are the only reversals of the anterior articulation of the maxilla with the jugal and Jinzhousaurus yangi is the only ankylopollexian taxon to possess a single ventral process of the predentary (Appendix 3). The character uniquely recovered by the analysis in PAUP is absence of parietal contact with the prootic (49:1), a homoplastic trait that ankylopollexians share with the outgroup, Lesothosaurus diagnosticus.

Finally, the unambiguous character transformations recovered by both analyses are a thickened dorsal edge of the ilium above the ischial peduncle (87:1), a dorsally arched ischium (92:1), and a rounded ischial shaft (93:1). All three of these traits are shared throughout the clade, with the exception of a derived and a reversed character in hadrosaurids (87:2 and 92:0, respectively) and a reversal in Jinzhousaurus yangi (92:0). These traits would seem to indicate the importance of pelvic morphology in identifying ankylopollexian taxa.

Styracosterna. The clade Styracosterna is very well supported in this analysis, with the highest Bremer, bootstrap, and GC support of any node (Figure 62, node C). Six unambiguous transformations support this node in the PAUP analysis, one character is uniquely recovered in the TNT analysis, and ten support the clade in both. In this analysis, Styracosterna are defined as Eolambia caroljonesa, Corythosaurus casuarius, their most 
recent common ancestor, and all of its descendants.

The characters recovered in PAUP alone are the presence of a maxillary diastema (31:1), a lack of supraoccipital participation in the foramen magnum due to the exoccipital (43:1), the presence of a dentary diastema (51:1), the dentary symphysis set below the tooth row (53:1), a denticulate occlusal margin of the predentary (62:1), and a triangular femoral fourth trochanter $(97: 1)$. The character recovered only in TNT is the femoral extensor groove forming a partially enclosed channel (98:2).

Among these characters, a lack of supraoccipital participation in the foramen magnum is a homoplastic reversal of the condition present in Zalmoxes robustus, Theiophytalia kerri, Dryosaurus altus, and Cumnoria prestwichii, to the state in Tenontosaurus and Thescelosaurus neglectus. A dentary diastema unites all styracosternans, save Iguanodon bernissartensis and Ouranosaurus nigeriensis. In addition, all members of the clade possess a dentary symphysis below the tooth row and a denticulate oral margin of the predentary, the latter of which they share with Tenontosaurus. Finally, all members of the clade below node $\mathrm{E}$ (Figure 7) possess a triangular fourth trochanter of the femur.

The characters recovered in both analyses are the presence of a lateral process of the maxilla for articulation with the jugal (32:1); the presence of a groove in the ventral surangular to receive the angular (64:1); ridges present on the maxillary and dentary teeth (70:2); hatchet-like sternals (77:1); short and block-like first metacarpals (79:1); closely appressed second through fourth metacarpals (80:1); a conical ungual of the first manual digit (82:1); flattened, twisted, and hoof-like second and third manual unguals (83:1); a short constriction followed by a distal expansion of the prepubic process of the pubis (90:2); and elongate but bluntly truncated pedal unguals (101:1).

All styracosternans share a lateral process of the maxilla for the jugal, hatchet-like sternals, and a similar morphology of the second and third manual unguals. In addition, all members of the clade, save hadrosaurids (Figure 7 , node $F$, defined below), share short and block-like first metacarpals; a conical ungual of the first manual digit; and elongate, bluntly truncated pedal unguals. Styracosternans basal to node E (Figure 7, discussed below) share a groove on the ventral surangular to articulate with the angular, while those basal to node $D$ (Hadrosauroidea, defined below) share ridges on maxillary and dentary teeth and closely appressed second through fourth metacarpals. Finally, the prepubic process of the pubis is variable within the clade.

Hadrosauroidea. The clade Hadrosauroidea (Figure 7 , node $D$ ) is recovered in this analysis with a Bremer support of four. Three unambiguous transformations uniquely support this node in the analysis run in PAUP, while one provides support only in the analysis run in TNT. Two unambiguous character optimizations were commonly recovered. Hadrosauroidea are defined herein as Probactrosaurus gobiensis, Corythosaurus casuarius, their most recent common ancestor, and all of its descendants.

The characters recovered in PAUP are a parietal twice as long as wide (46:3); appressed, slender, and elongate second through fourth metacarpals (80:2); and posteriorly and anteriorly expanded distal condyles of the femur (99:1). The analysis in TNT recovered the absence of the coronoid bone at this node (57:1). Finally, both analyses recovered an axially flared coronoid process $(60: 1)$ and the presence of ridges on the maxillary teeth with reduced ridges on the dentary teeth (70:3). Although all of these character states are synapomorphic for this clade, there are a few deviations. The ratio of the length to the width of the parietal and an axially flared coronoid process are not shared with Telmatosaurus transsylvanicus. Metacarpal morphology and the expanded condyles of the femur are shared among all taxa in this clade, although the former is unknown in T. transsylvanicus and both are unknown in Protohadros byrdi. The absence of the coronoid is shared among all members of the clade, although the character state is unknown in Probactrosaurus gobiensis. Finally, tooth morphology unites the clade, although T. transsylvanicus and Edmontosaurus regalis possess a derived state.

Node E. The hadrosauroid clade, excluding Probactrosaurus gobiensis and Protohadros byrdi (Figure 7, node E), is also well supported by these analyses, with bootstrap and GC values of 81 and 83 , respectively. Although this clade is well supported and does not have a clear correlate in other analyses (e.g., Prieto-Márquez, 2010), the ingroup of the present analysis is too cursory and representative to justify the erection of a novel clade. The node is supported by two unambiguous character transformations unique to the TNT analysis and by two shared by both analyses.

The character transformations unique to the analysis conducted in TNT are a fourth trochanter of the femur shaped as a crested eminence (97:2) 
and a fully enclosed femoral extensor groove (98:3). The character transformations recovered in both analyses for this node are an anteriorly deflected coronoid process of the mandible (56:2) and a lack of surangular foramina (69:3). Each of these states is synapomorphic.

Hadrosauridae. The clade Hadrosauridae (Figure 7 , node F) is nearly as well supported as Styracosterna (Figure 7, node $\mathrm{C}$ ). In this analysis, Hadrosauridae are defined as Edmontosaurus regalis, Corythosaurus casuarius, their most recent common ancestor, and all of its descendants. The clade is supported by two unambiguous character transformations unique to the analysis run in PAUP and by five shared by both analyses.

In the analysis conducted in PAUP, Hadrosauridae are supported by the presence of a ventral lobe of the jugal (21:1) and a laterally reflected acromial boss of the scapula $(75: 1)$. The characters which support the node in both analyses are a narrow ventral quadratic condyle (38:1), half of the dentary bearing dentition (55:2), the presence of two processes around the Meckelian canal (58:1), lack of lateral visibility of the angular (63:2), and the angular laterally abutting the surangular $(64: 2)$.

Among these characters, the ventral lobe of the jugal is homoplastic with Iguanodon bernissartensis, and the laterally reflected acromial boss of the scapula is homoplastic with the genus Tenontosaurus and with Zalmoxes robustus. The narrow ventral quadratic condyle, the dentition restricted to half of the dentary, two processes around the Meckelian canal, the lack of lateral visibility of the angular, and the angular laterally abutting the surangular are all autapomorphic for hadrosaurids. Eolambia caroljonesa and Jinzhousaurus yangi. The North American taxon, Eolambia caroljonesa (Kirkland, 1998; Head, 2001; McDonald et al., 2012) is recovered in this analysis as the sister taxon of Jinzhousaurus yangi, an older species from Asia (Wang and Xu, 2001; Wang et al., 2011). Although McDonald (2012) recovered E. caroljonesa as the sister taxon of another Asian species (Probactrosaurus gobiensis), it is interesting to note that the North American taxon is once again resolved with Asian affinities. In addition, the placement of $E$. caroljonesa and J. yangi as basal to Iguanodon bernissartensis and Mantellisaurus atherfieldensis is unusual, as the former taxa are generally recovered as more closely related to the hadrosaurids (e.g., McDonald et al., 2010; McDonald, 2012). The basal position of these taxa when compared to previous analyses may be influenced, in part, by the relatively recent description of their postcranial anatomy (Wang et al., 2011; McDonald et al., 2012).

The node uniting Eolambia caroljonesa and Jinzhousaurus yangi is supported by three unambiguous character optimizations in both PAUP and TNT. The character states which unite these taxa are the nasal process of the premaxilla extending back to the posterior border of the dorsal process of the maxilla ( $4: 1$; shared with Zalmoxes robustus and Corythosaurus casuarius), presence of the triangular process of the parietal (9:0; shared with Probactrosaurus gobiensis), and absence of parietal contact with the postorbital (17:1; shared with Z. robustus). Each of these character states optimizes in additional locations on the tree, for other taxa, implying homoplasy.

Tenontosaurus. The monophyletic genus Tenontosaurus, with members $T$. dossi and $T$. tilletti, is recovered in a position very similar to that in previous work (e.g., Norman, 2004; Butler et al., 2008; McDonald, 2012), with a Bremer support of five and high bootstrap and GC support values (Figure 7 , node G). Six unambiguous character optimizations were recovered in the analysis conducted in PAUP, and an additional character was commonly recovered by both. The two species of the genus share over $90 \%$ of their character states, although $T$. dossi is less completely known and may differ in unknown ways from T. tilletti (Appendix 3).

The following characters were recovered as unambiguous only in the analysis conducted in PAUP: the nasal process of the premaxilla overlaps the nasal (13:1), the postorbital process of the jugal is the posteriormost extent of that element (22:1), the parietal appears triangular in dorsal aspect $(45: 1)$, the oral margin of the predentary is denticulate (62:1), the scapular acromial boss is reflected laterally (75:1), and the obturator process of the ischium is present near the middle of the ischial shaft $(94: 1)$. Although these character states were recovered as support for this node by the analysis performed in TNT as well (Figure 62, node G), most are ambiguous (that is, they resolve in other locations on the tree), with no clear distribution pattern among other taxa. However, one of the character states, the denticulate oral margin of the predentary, is shared with all other iguanodontians except Dryosaurus altus and Zalmoxes robustus (Appendix 3). The character state recovered in both analyses is a laterally compressed and barlike prepubic process of the pubis (90:1).

Tenontosaurus tilletti. Synapomorphic characters of the clade Iguanodontia, of which Tenontosaurus tilletti is the basalmost member, and of the genus 
Tenontosaurus are discussed in the preceding sections (see Results sections on Iguanodontia and Tenontosaurus, above).

Plesiomorphies for Tenontosaurus tilletti, which are listed in the foregoing diagnosis (see Systematic Paleontology section above), are discussed below. Although the lack of contact between the jugal and the quadrate in T. tilletti $(20: 0)$ is presumably plesiomorphic, most taxa analyzed here share this character, including some of the most derived (e.g., Corythosaurus casuarius). Contact between the two elements is acquired by only four taxa: Dryosaurus altus, Theiophytalia kerri, Jinzhousaurus yangi, and Edmontosaurus regalis (Appendix 3). Tenontosaurus tilletti also lacks a quadrate foramen (25:0), a characteristic shared with most other ingroup taxa (Appendix 3, e.g., Protohadros byrdi and Edmontosaurus regalis), except $D$. altus, Mantellisaurus atherfieldensis, Iguanodon bernissartensis, J. yangi, and Ouranosaurus nigeriensis, which all possess a quadrate foramen (Appendix 3). Tenontosaurus tilletti shares a large quadratojugal (26:1); contact between the nasal and the lacrimal (28:0); an articulation of the jugal with the posterior maxilla (33:0); lack of a quadrate notch (37:0); parietal and prootic that do not make contact (49:1); and, postcranially, a thin and beveled dorsal edge of the ilium above the ischial peduncle (87:0); and, finally, a straight (92:0) and flattened (93:0) shaft of the ischium with more basal taxa, with few exceptions (Appendix 3 ).

Finally, the following suite of characters is autapomorphic for Tenontosaurus tilletti. Members of the taxon lack premaxillary teeth (3:1), a feature shared with all more derived iguanodontian taxa (Figure 7, Appendix 3). However, because the sister taxon, T. dossi, retains the plesiomorphic premaxillary teeth, this characteristic becomes autapomorphic for the species. The length of the suture between the frontals and the parietal of $T$. tilletti is equal to about two-thirds the combined width of the frontals (Figure 62, homoplastic character shared with Telmatosaurus transsylvanicus, Appendix 3: 7:1) and the length of the nasals is one and one-half times the length of the frontals (10:1). Postcranially, the scapular blade of $T$. tilletti is posteriorly curved and distally flared (74:2), the distal phalanx of the second manual digit is clawlike while that of the third is a nub (83:2), and the femoral shaft is straight $(96: 1)$.

\section{CONCLUSION}

The unique preservation of OMNH 58340 and the CT data gathered from it allow emendation of several cranial features proposed by Ostrom (1970, p. 86) in the original diagnosis of Tenontosaurus tilletti (see revised cranial diagnosis above). These include the nearly triangular, rather than subrectangular, borders of the orbit (Figure 2), the single, rather than double, coronoid process of the mandible (Figure 46), the relatively straight retroarticular process of the mandible (Figure 46; Ostrom, 1970 , figure 8), and the bilaterally enameled teeth (Figure 60). Beyond this, several apparent, although incorrect, features of the specimens described that were not part of the formal diagnosis were included in the description and illustrations of the species (Ostrom, 1970). These include the exaggerated height of the maxilla and nasal in lateral aspect (Ostrom, 1970, figure 8), the contribution of the jugal to the accessory lateral fenestra (Ostrom, 1970, figure 8), and the sharp posterior concavity of the paroccipital processes (Ostrom, 1970 , figure 9). Each of these is emended in the present study.

The two species within Tenontosaurus are retained, and specimens from the Antlers Formation of Oklahoma are assigned to T. tilletti on the basis of consensus in all characters coded in the preceding phylogenetic analysis as well as characteristics outside of the analysis included in Table 1. These characteristics are based upon the work of Winkler et al. (1997) in their description and definition of the sister species, $T$. dossi, as well as from personal observations of specimens of both species from their known ranges.

With regard to the phylogenetic position of the genus Tenontosaurus, Norman suggested (1986, p. 330) that several apomorphic characteristics of the taxon are a result of parallel or convergent evolution in large-bodied ornithopods (no premaxillary teeth in T. tilletti, crested 4th trochanter in the middle of the femur and a laterally compressed lesser trochanter on the same element, a deep anterior pubic process and a short posterior pubic process, and a phalanx lost in manual digit III). The genus also retains several plesiomorphic features, such as a lack of a prominent ridge on the maxillary teeth, a relatively enlarged jugal, and, in $T$. dossi, the presence of premaxillary teeth. Moreover, some characteristics that Forster (1990) argued place Tenontosaurus in closer association with iguanodontians, such as paired anterior processes of the maxilla and a small antorbital fenestra, are invalidated by this study. Finally, Tenontosaurus occurs late (Aptian-Albian) compared to Dryosaurus altus (Kimmeridgian-Tithonian); and Theiophytalia kerri, Mantellisaurus atherfieldensis and 
THOMAS: SKULL OF TENONTOSAURUS

\begin{tabular}{|c|c|c|c|c|}
\hline Characteristic & $\begin{array}{l}\begin{array}{l}\text { Tenontosaurus } \\
\text { tilletti }\end{array} \\
\end{array}$ & \begin{tabular}{|l|}
$\begin{array}{l}\text { Antlers Formation } \\
\text { specimens }\end{array}$ \\
\end{tabular} & $\begin{array}{l}\begin{array}{l}\text { Tenontosaurus } \\
\text { dossi }\end{array} \\
\end{array}$ & Interpretation \\
\hline Premaxillary Teeth & Absent & Absent & Present & \\
\hline Tooth Count & $\begin{array}{l}9-13 \text { maxillary and } \\
10-13 \text { dentary tooth } \\
\text { positions }\end{array}$ & $\begin{array}{l}11-13 \text { maxillary and } \\
12-14 \text { dentary tooth } \\
\text { positions }\end{array}$ & $\begin{array}{c}11-12 \text { tooth positions } \\
\text { in both the maxilla and } \\
\text { the dentary }\end{array}$ & $\begin{array}{l}\text { Appears ontogenetically related. Smaller individuals } \\
\text { from both the Antlers and the Cloverly have fewer } \\
\text { alveoli. It is interesting to note that similarly sized } \\
\text { individuals of } \mathrm{T} \text {. dossi have fewer teeth than Cloverly or } \\
\text { Antlers tenontosaurs. }\end{array}$ \\
\hline Tooth Morphology & & & & $\begin{array}{l}\text { Similar between species; keeled Dentary and ridged } \\
\text { Maxillary Teeth }\end{array}$ \\
\hline Anteromedial Maxillary Process & & & & Similar between species \\
\hline Facial Lamina of Maxilla & $\begin{array}{l}\text { Expanded posteriorly } \\
\text { above the antorbital } \\
\text { fenestra }\end{array}$ & $\begin{array}{c}\text { Expanded posteriorly } \\
\text { above the antorbital } \\
\text { fenestra }\end{array}$ & $\begin{array}{c}\text { Not expanded } \\
\text { posteriorly above the } \\
\text { antorbital fenestra }\end{array}$ & \\
\hline Premaxillary Contact with the Lacrimal & & & & $\begin{array}{l}\text { Similar between species; Premaxilla contacts the } \\
\text { Lacrimal posteriorly }\end{array}$ \\
\hline Shape of the Antorbital Fenestra & Slit-like & Slit-like & Reniform & Variable? \\
\hline $\begin{array}{l}\text { Nature of Nasal, Prefrontal, Frontal } \\
\text { articulation }\end{array}$ & Unknown & $\begin{array}{l}\text { Nasals overlap } \\
\text { Frontals, overlapped } \\
\text { by Prefrontals }\end{array}$ & $\begin{array}{l}\text { Nasals overlap } \\
\text { Frontals, overlapped } \\
\text { by Prefrontals }\end{array}$ & \\
\hline Quadrate Morphology & & & & Similar between species \\
\hline Palatine Morphology & & & & Similar between species \\
\hline Jugal Morphology & & & & Similar between species \\
\hline Quadratojugal Foramen & & & & Similar between species \\
\hline Contact between Prootic and Parietal & Unknown & No contact & No contact & \\
\hline $\begin{array}{l}\text { Participation of the Supraoccipital in } \\
\text { the Foramen Magnum }\end{array}$ & & & & Similar between species \\
\hline Supraoccipital Morphology & Unknown & \begin{tabular}{|c|} 
Visible on the \\
dorsolateral braincase
\end{tabular} & $\begin{array}{c}\text { Visible on the } \\
\text { dorsolateral braincase }\end{array}$ & \\
\hline Facial Nerve (CN VII) Foramen & Unknown & Single, undivided & Single, undivided & \\
\hline $\begin{array}{l}\text { Angle of the Crista Tuberalis relative to } \\
\text { the Parietal }\end{array}$ & Unknown & $65^{\circ}$ & $55^{\circ}$ & \\
\hline $\begin{array}{l}\text { Angle of the base of the } B \text { raincase } \\
\text { relative to the long axis of the } \\
\text { Drainnace. }\end{array}$ & & & & Similar between species \\
\hline Ventral Process of the Predentary & & & & Similar between species; weakly indented \\
\hline Predentary cusps & $\begin{array}{c}\text { One central flanked by } \\
\text { three lateral pairs }\end{array}$ & $\begin{array}{c}\text { One central flanked by } \\
\text { three lateral pairs }\end{array}$ & $\begin{array}{c}\text { One central flanked by } \\
\text { a single pair of large } \\
\text { lateral denticles }\end{array}$ & \\
\hline Cervical Vertebral Morphology & & & & Similar between species \\
\hline Dorsal Vertebral Morphology & & & & Similar between species \\
\hline Sacral Vertebral Morphology & Some centra unfused & All centra fused & All centra fused & Similar between species; fusion possibly ontogenetic \\
\hline Caudal Vertebral Morphology & \begin{tabular}{|c|} 
Straight to anteriorly \\
concave neural spines \\
on proximal caudals; \\
middle caudals less \\
posteriorly concave \\
\end{tabular} & $\begin{array}{c}\text { Concave neural spines } \\
\text { on proximal caudals; } \\
\text { middle caudals } \\
\text { unknown }\end{array}$ & $\begin{array}{l}\text { Strongly concave } \\
\text { neural spines on } \\
\text { proximal caudals; } \\
\text { middle caudals more } \\
\text { posteriorly concave }\end{array}$ & Variable between individuals \\
\hline Hypaxial Tendons & Single Layered Sheet & Single Layered Sheet & $\begin{array}{l}\text { Run in distinct } \\
\text { hundlos }\end{array}$ & \\
\hline Scapular Morphology & $\begin{array}{c}\text { Straight anterior } \\
\text { border }\end{array}$ & $\begin{array}{l}\text { Straight anterior } \\
\text { border }\end{array}$ & $\begin{array}{c}\text { Flares, resulting in } \\
\text { concave anterior edge }\end{array}$ & \\
\hline Coracoid Morphology & $\begin{array}{l}\text { Overall oblong } \\
\text { appearance }\end{array}$ & $\begin{array}{c}\text { Overall square } \\
\text { appearance, but with } \\
\text { deeper ventral arch } \\
\text { between the glenoid } \\
\text { fossa and sternal } \\
\text { process } \\
\end{array}$ & $\begin{array}{l}\text { Overall square } \\
\text { appearance }\end{array}$ & Similar between species, despite slight differences \\
\hline Sternal Morphology & Large, lunate & Large, lunate & Large, lunate & Similar between species \\
\hline Humeral Morphology & & & & Similar between species \\
\hline Radial and Ulnar Morphology & & & & Similar between species \\
\hline Carpal Morphology & $\begin{array}{l}\text { Ulnare and } \\
\text { intermedium less } \\
\text { tightly articulated; } \\
\text { radiale with distinct } \\
\text { shaft }\end{array}$ & $\begin{array}{l}\text { Uinare and } \\
\text { intermedium less } \\
\text { tightly articulated; } \\
\text { radiale larger and with } \\
\text { reduced shaft }\end{array}$ & $\begin{array}{l}\text { Untermede and } \\
\text { interlocked, tightly } \\
\text { tongue-and-groove } \\
\text { joint; radiale larger } \\
\text { and with reduced }\end{array}$ & $\begin{array}{l}\text { Same three elements shared between species: radiale, } \\
\text { intermedium, and ulnare }\end{array}$ \\
\hline Metacarpal V articular surface & \begin{tabular}{|l} 
Less extensive dorsal \\
surface, presumably \\
lesser hyperextension
\end{tabular} & \begin{tabular}{|c|} 
Less extensive dorsal \\
surface, presumably \\
lesser hyperextension
\end{tabular} & $\begin{array}{c}\text { Extensive dorsal } \\
\text { surface, indicating } \\
\text { greater } \\
\text { hyperextension }\end{array}$ & \\
\hline Manual Phalangeal Morphology & & & & Similar between species \\
\hline Iliac Morphology & $\begin{array}{l}\text { Weak lateral brevis } \\
\text { shelf }\end{array}$ & \begin{tabular}{|c|}
$\begin{array}{c}\text { Weak lateral brevis } \\
\text { shelf, anterior process } \\
\text { turned laterally }\end{array}$ \\
\end{tabular} & $\begin{array}{c}\text { Weak lateral brevis } \\
\text { shelf; anterior process } \\
\text { turned laterally }\end{array}$ & Similar between species \\
\hline Ischial Morphology & $\begin{array}{c}\text { Tab-like obturator } \\
\text { process in some } \\
\text { individuals (e.g. } \\
\text { AMNH } 3040 \text {, OMNH } \\
\text { 10132); Obturator } \\
\text { processes low ridges } \\
\text { with tapering ends in } \\
\text { other individuals (e.g., } \\
\text { OMNH 10144, OMNH } \\
34785 \text { ) } \\
\end{array}$ & $\begin{array}{l}\text { Obturator processes } \\
\text { low ridges with } \\
\text { tapering ends } \\
\end{array}$ & $\begin{array}{l}\text { Obturator processes } \\
\text { low ridges with } \\
\text { tapering ends }\end{array}$ & Variable between individuals; possibly ontogenetic \\
\hline Pubic Morphology & $\begin{array}{l}\text { Prepubic process with } \\
\text { bulge in ventral } \\
\text { border; prepubic } \\
\text { process relatively long; } \\
\text { postpubic process } \\
\text { shorter than ischium }\end{array}$ & \begin{tabular}{|c|} 
Prepubic process with \\
bulge in ventral border; \\
prepubic process \\
relatively long; \\
postpubic process \\
shorter than ischium
\end{tabular} & $\begin{array}{l}\text { Prepubic process } \\
\text { dorsally concave and } \\
\text { ventrally convex; } \\
\text { relatively short } \\
\text { prepubic process; } \\
\text { postibubic process } \\
\text { possibly subequal to } \\
\text { ischium in length } \\
\end{array}$ & \\
\hline Femoral Morphology & & & & Similar between species \\
\hline Length of Femur relative to Tibia & $\begin{array}{c}\text { Femur longer than the } \\
\text { tibia }\end{array}$ & $\begin{array}{c}\text { Femur longer than the } \\
\text { tibia }\end{array}$ & $\begin{array}{l}\text { Tibia slightly longer } \\
\text { than femur }\end{array}$ & Possibly ontogenetic \\
\hline Tibial and Fibular Morphology & & & & Similar between species \\
\hline Tarsal Morphology & & & & Similar between species \\
\hline Metatarsal Morphology & $\begin{array}{l}\text { Metatarsal V relatively } \\
\text { short (<30\% length of } \\
\text { Metatarsal III) }\end{array}$ & $\begin{array}{l}\text { Metatarsal V relatively } \\
\text { short }(<30 \% \text { length of } \\
\text { Metatarsal III) }\end{array}$ & $\begin{array}{l}\text { Metatarsal } V \text { relatively } \\
\text { long (>30\% length of } \\
\text { Metatarsal III) }\end{array}$ & \\
\hline
\end{tabular}


TABLE 1. Comparisons of non-coded characteristics between the two species of Tenontosaurus from their originally described ranges ( $T$. tilletti from Montana, USA and T. dossi from Texas, USA) and specimens of T. tilletti from the Antlers Formation of Oklahoma. Short descriptors of each characteristic are listed in the first column, and explanations of those characteristics from Tenontosaurus specimens in each of the three known ranges are listed in the second through fourth columns. A short interpretation is included in the fifth column with additional information. Cells colored in blue represent commonalities between one or both of the known Tenontosaurus species with individuals from the Antlers Formation, cells colored in yellow represent differences, and cells colored in red represent unknowns. This table is based off of observations from Ostrom (1970), Forster (1990), and Winkler et al. (1997), as well as personal observations made by the author. (Table on previous page.)

Iguanodon bernissartensis (Aptian-Albian), all of which resolve higher in the iguanodontian tree (Figure 7), as observed by Winkler et al. (1998). These lines of evidence support the hypothesis that Tenontosaurus may very well have been a late, convergent offshoot from more basal ornithopod ancestors, and may constitute a sister taxon to Iguanodontia rather than a direct precursor in the evolutionary lineage from basal ornithopods to hadrosaurs.

Conversely, although plesiomorphic, the relatively smooth maxillary teeth (Appendix 3: 70:1) affiliate Tenontosaurus with Dryosaurus altus and Theiophytalia, two firmly established iguanodontians, as noted. Also of note are the roughly parallel dorsal and ventral borders of the dentary, which T. tilletti (Figure 49) shares with most iguanodontians (Figure 62, node A, Appendix 3: 54:1). Finally, the clade Iguanodontia, with Tenontosaurus as its basalmost member, is well supported with six synapomorphies (see results section above, Appendices 2 and 3 .

Ultimately, the overall effect of the anatomy of Tenontosaurus is both ambivalent and intriguing, relating the genus to more basal or more derived taxa depending upon the features examined. Accordingly, the present study agrees with Winkler et al. (1997), who declared the placement of the genus within Iguanodontia to be ambiguous at best and recommended caution when assigning the genus to a particular clade in future analyses. The character changes that occur between Thescelosaurus neglectus and Tenontosaurus (Figure 7, node A) and those that occur between Tenontosaurus and Zalmoxes robustus (Figure 7, node F) are, therefore, deemed equivocal, and I will conclude on the side of conservation, retaining the genus Tenontosaurus as the most basal of the iguanodontians examined in this study. In addition, both species of Tenontosaurus possess the characters used to define the clade Iguanodontia by Norman (2004, p. 413), which allows that diagnosis of the clade to stand.
The similarity between the two species of the genus-Tenontosaurus dossi and T. tilletti-is striking, both in appearance and in their resolution in phylogenetic analyses (e.g., McDonald (2012) consistently recovered Tenontosaurus in all stages of his analysis, even though the rest of the cladogram may have been unresolved). Very few characteristics distinguish the two taxa (absence of premaxillary teeth in T. tilletti: $3: 1$; different relative lengths of the suture of the frontals with the parietal: $7: 1$; differing maxillary tooth count: $30: 13$; curved and distally flared scapular blade in T. tilletti: 74:2; and differing unguals in manus digits II and III: 83:2), and future research and discovery could very well collapse the species of the genus, although no such data is presently at hand (Table 1). As noted by Winkler et al. (1997), anatomical comparisons between the two species facilitate an enlightening glimpse into the evolutionary trajectory of the genus, as well as the larger clade Iguanodontia. A more thorough analysis of the systematic differences between the taxa is certainly warranted.

In order to further elucidate the affinities of basal ornithopod and iguanodontian taxa, as well as the origins of the derived hadrosaurids, additional analysis is crucial, as a more rigorous and thorough phylogenetic analysis of Ornithopoda is beyond the scope of this study. It is recommended that future analyses work to produce a major reevaluation of the systematics of Ornithopoda, in particular trying to elucidate the position of Tenontosaurus with respect to the rest of Iguanodontia. The apparent phylogenetic lability of several taxa (including Zalmoxes robustus, Eolambia caroljonesa, Jinzhousaurus yangi, and Ouranosaurus nigeriensis, among others), in comparison with their positions in previous analyses (e.g., Norman, 2004; McDonald, 2011), emphasizes the need for such a reevaluation in future research. 


\section{ACKNOWLEDGMENTS}

Special thanks to the members of my MS committee, Drs. R.C., C.G., N.C., and S.W. Thanks also to K.D. for his time, patience, and expertise. Thanks also to the faculty and staff of the Department of Biology at the University of Oklahoma and the Sam Noble Oklahoma Museum of Natural History. Special thanks to the High-Resolution X-ray Computed Tomography Facility at the University of Texas at Austin for scanning and processing the specimens used in this study. Finally, most sincere gratitude to my wife, K.T., and all of my family, who have supported me.

Funding for this research was provided by the Loren G. Hill Zoology Excellence Fund, the Robberson Research and Creative Endeavors Grant, the Graduate Student Senate, and the M. Blanche Adams and M. Frances Adams Memorial Scholarships in Zoology Fund.

\section{REFERENCES}

Barrett, P.M., Butler, R.J., Wang, X.-L., and Xing, X. 2009. Cranial anatomy of the iguanodontoid ornithopod Jinzhousaurus yangi from the Lower Cretaceous Yixian Formation of China. Acta Palaeontologica Polonica, 54:35-48.

Boulenger, G.A. 1881. Sur l'arc pelvien chez les dinosauriens de Bernissart. Bulletin de l'Academie Royale de Belgique, 3 sèrie, 1:600-608.

Bremer, K. 1994. Branch support and tree stability. Cladistics, 10:295-304.

Brill, K. and Carpenter, K. 2006. A description of a new ornithopod from the Lytle Member of the Purgatoire Formation (Lower Cretaceous) and a reassessment of the skull of Camptosaurus, p. 49-67. In Carpenter, K. (ed.), Horns and Beaks: Ceratopsian and Ornithopod Dinosaurs. Indiana University Press, Bloomington, Indiana.

Brown, B. 1914. Anchiceratops, a new genus of horned dinosaurs from the Edmonton Cretaceous of Alberta. With discussion of the origin of the ceratopsian crest and the brain casts of Anchiceratops and Trachodon. Bulletin of the American Museum of Natural History, 33:539-548.

Brown, B. 1916. Corythosaurus casuarius: skeleton, musculature and epidermis. Bulletin of the American Museum of Natural History. Published by order of the Trustees, American Museum of Natural History, 35:701-716.

Brown, C.M., Evans, D.C., Ryan, M.J., and Russell, A.P. 2013. New data on the diversity and abundance of small-bodied ornithopods (Dinosauria, Ornithischia) from the Belly River Group (Campanian) of Alberta. Journal of Vertebrate Paleontology, 33:495-520.
Butler, R.J., Upchurch, P., and Norman, D.B. 2008. The phylogeny of the ornithischian dinosaurs. Journal of Systematic Palaeontology, 6:1-40.

Cifelli, R.L., Gardner, J.D., Nydam, R.L., and Brinkman, D.L. 1997. Additions to the vertebrate fauna of the Antlers Formation (Lower Cretaceous), southern Oklahoma. Oklahoma Geology Notes, Oklahoma Geological Survey, 57:124-131.

Cohen, B. and Raphan, T. 2004. The physiology of the vestibuloocular reflex, p. 235-285. In Highstein, S., Fay, R., and Popper, A. (eds.), The Vestibular System. Springer, New York, New York.

Crompton, A.W. and Charig, A.J. 1962. A new ornithischian from the Upper Triassic of South Africa. Nature, 196:1074-1077.

Davis, B.M, and Cifelli, R.L. 2011. Reappraisal of the tribosphenidan mammals from the Trinity Group (Aptian-Albian) of Texas and Oklahoma. Acta Palaeontologica Polonica, 56:441-462.

Davis, B.M., Cifelli, R.L., and Kielan-Jaworowska, Z. 2008. Earliest evidence of Deltatheroidea (Mammalia: Metatheria) from the Early Cretaceous of North America, p. 3-24. In Sargis, E.J. and Dagosto, M. (eds.), Mammalian Evolutionary Morphology: A Tribute to Frederick S. Szalay. Springer, Dordrecht, The Netherlands.

Dollo, L. 1888. Iguanodontidae et Camptonotidae. Comptes rendus de l'Académie des Sciences, 106:775-777.

Eddy, D.R. and Clarke, J.A. 2011. New information on the cranial anatomy of Acrocanthosaurus atokensis and its implications for the phylogeny of Allosauroidea (Dinosauria: Theropoda). PLoS ONE, 6:e17932.

Edmund, A.G. 1957. On the special foramina in the jaws of ornithischian dinosaurs. Contributions of the Royal Ontario Museum, Division of Zoology and Palaeontology, 48:3-14.

Edmund, A.G. 1960. Tooth replacement phenomena in the lower vertebrates. Contributions of the Life Sciences Division of the Royal Ontario Museum, 52:1190.

Forster, C.A. 1985. A description of the postcranial skeleton of the Early Cretaceous ornithopod Tenontosaurus tilletti, Cloverly Formation, Montana and Wyoming. Unpublished MS Thesis, University of Pennsylvania at Philadelphia, Pennsylvania, USA.

Forster, C.A. 1990. The postcranial skeleton of the ornithopod dinosaur Tenontosaurus tilletti. Journal of Vertebrate Paleontology, 10:273-294.

Franzosa, J. and Rowe, T. 2005. Cranial endocast of the Cretaceous theropod dinosaur Acrocanthosaurus atokensis. Journal of Vertebrate Paleontology, 25:859-864.

Galton, P.M. 1973. Redescription of the skull and mandible of Parksosaurus from the Late Cretaceous with comments of the family Hypsilophodontidae (Ornithischia). Life Sciences Contribution, Royal Ontario Museum, 89:1-21. 
Galton, P.M. 1974a. The ornithischian dinosaur Hypsilophodon from the Wealden of the Isle of Wight. Bulletin of the British Museum of Natural History (Geology), 25:1-152.

Galton, P.M. 1974b. Notes on Thescelosaurus, a conservative ornithopod dinosaur from the Upper Cretaceous of North America, with comments on ornithopod classification. Journal of Paleontology, 48:1048-1067.

Galton, P.M. 1978. Fabrosauridae, the basal family of ornithischian dinosaurs (Reptilia: Ornithopoda). Paläontologische Zeitschrift, 52:138-159.

Galton, P.M. 1983. The cranial anatomy of Dryosaurus, a hypsilophodontid dinosaur from the Upper Jurassic of North America and East Africa, with a review of hypsilophodontids from the Upper Jurassic of North America. Geologica et Palaeontologica, 17:207-243.

Galton, P.M. 1989. Crania and endocranial casts from ornithopod dinosaurs of the families Dryosauridae and Hypsilophodontidae (Reptilia: Ornithischia). Geologica et Palaeontologica, 23:217-239.

Galton, P.M. 1995. The species of the basal hypsilophodontid dinosaur Thescelosaurus Gilmore (Ornithischia: Ornithopoda) from the Late Cretaceous of North America. Neues Jahrbuch für Geologie und Paläontologie Abhandlungen, 198:297-311.

Galton, P.M. 1997. Cranial anatomy of the basal hypsilophodontid dinosaur Thescelosaurus neglectus Gilmore (Ornithischia: Ornithopoda) from the Upper Cretaceous of North America. Revue de Paléobiologie, 16:231-258.

Galton, P.M. and Powell, H.P. 1980. The ornithischian dinosaur Camptosaurus prestwichii from the Upper Jurassic of England. Palaeontology, 23:411-443.

Gilmore, C. 1909. Osteology of the Jurassic reptile Camptosaurus, with revision of the species of the genus and description of two new species. Proceedings of the United States National Museum, 36:197332.

Gilmore, C.W. 1913. A new dinosaur from the Lance Formation of Wyoming. Smithsonian Miscellaneous Collections, 61:1-5.

Gilmore, C.W. 1915. Osteology of Thescelosaurus, an orthopodous dinosaur from the Lance Formation of Wyoming. Proceedings of the United States National Museum, 49:591-627.

Goloboff, P.A., Farris, J.S., Källersjö, M., Oxelman, B., Ramírez, M.J., and Szumik, C. 2003. Improvements to resampling measures of group support. Cladistics, 19:324-332.

Goloboff, P.A., Farris, J.S., and Nixon, K.C. 2008. TNT, a free program for phylogenetic analysis. Cladistics, 24:774-786.

Graf, W. and Klam, F. 2006. Le système vestibulaire: anatomie fonctionnelle et comparée, évolution et développement. Comptes Rendus Palevol, 5:637655.
Head, J. 1998. A new species of basal Hadrosaurid (Dinosauria, Ornithischia) from the Cenomanian of Texas. Journal of Vertebrate Paleontology, 18:718738.

Head, J.J. 2001. A reanalysis of the phylogenetic position of Eolambia caroljonesa (Dinosauria, Iguanodontia). Journal of Vertebrate Paleontology, 21:392-396.

Hobday, D., Woodruff, C., and McBride, M. 1981. Paleotopographic and structural controls on non-marine sedimentation of the Lower Cretaceous Antlers Formation and correlatives, north Texas and southeastern Oklahoma. Society of Economic Paleontologists and Mineralogists, Special Publication 31:71-87.

Hooley, R. 1925. On the skeleton of Iguanodon atherfieldensis sp. nov., from the Wealden Shales of Atherfield (Isle of Wight). Quarterly Journal of the Geological Society, 81:1-61.

Horner, J.R., Weishampel, D.B., and Forster, C.A. 2004. Hadrosauridae, p. 438-463. In Weishampel, D.B., Dodson, P., and Osmólska, H. (eds.), The Dinosauria, $2^{\text {nd }}$ ed. University of California Press, Berkeley, California.

Hulke, J. 1880. Iguanodon prestwichii, a new species from the Kimmeridge Clay, distinguished from I. mantelli of the Wealden Formation in the S.E. of England and Isle of Wight by differences in the shape of the vertebral centra, by fewer than five sacral vertebrae, by the simpler character of its tooth-serrature, etc., founded on numerous fossil remains lately discovered at Cumnor, near Oxford. Quarterly Journal of the Geological Society of London, 36:433-456.

Huxley, T.H. 1869. On Hypsilophodon, a new genus of Dinosauria. Proceedings of the Geological Society of London, 204:3-4.

Jacobs, L.L. and Winkler, D.A. 1998. Mammals, archosaurs, and the Early to Late Cretaceous transition in north-central Texas. In Tomida, Y., Flynn, L.J., and Jacobs, L.L. (eds.), Advances in Vertebrate Paleontology and Geochronology. National Science Museum Monographs, Tokyo, 14:253-280.

Kirkland, J.I. 1998. A new Hadrosaurid from the Upper Cedar Mountain Formation (Albian-Cenomanian: Cretaceous) of Eastern Utah - the oldest known Hadrosaurid (Lambeosaurine?). New Mexico Museum of Natural History and Science Bulletin, 14:283-295.

Knoll, F., Witmer, L.M., Ortega, F., Ridgely, R.C., and Schwarz-Wings, D. 2012. The braincase of the basal sauropod dinosaur Spinophorosaurus and 3D reconstructions of the cranial endocast and inner ear. PLOS ONE 7:e30060.

Lambe, L.M. 1917. A new genus and species of crestless hadrosaur from the Edmonton Formation of Alberta. Ottawa Naturalist, 31:65-73.

Langston, W. 1960. The vertebrate fauna of the Selma Formation of Alabama: Part VI: The Dinosaurs. Fieldiana: Geology Memoirs, 3:315-361.

Lull, R.S. and Wright, N.E. 1942. Hadrosaurian dinosaurs of North America. The Special Papers of the Geological Society of America, 40:1-242. 
Manley, F.H., Jr. 1965. Clay mineralogy and clay-mineral facies of the Lower Cretaceous Trinity Group, southern Oklahoma. Unpublished PhD Dissertation, University of Oklahoma, Norman, Oklahoma, USA.

Marsh, O. 1878. Principal characters of American Jurassic dinosaurs. Part I. American Journal of Science, series 3, 16:411-416.

Marsh, O. 1879. Notice of new Jurassic reptiles. American Journal of Science, series 3, 28:501-505.

Marsh, O. 1881. Principal characters of American Jurassic dinosaurs. Part V. American Journal of Science, series 3, 21:417-423.

McDonald, A.T. 2011. The taxonomy of species assigned to Camptosaurus (Dinosauria: Ornithopoda). Zootaxa, 2783:52-68.

McDonald, A.T. 2012. Phylogeny of basal iguanodonts (Dinosauria: Ornithischia): an update. PLoS ONE, 7: e36745.

McDonald, A.T., Bird, J., Kirkland, J. I., and Dodson, P. 2012. Osteology of the basal hadrosauroid Eolambia caroljonesa (Dinosauria: Ornithopoda) from the Cedar Mountain Formation of Utah. PLOS ONE 7: e45712.

McDonald, A.T., Kirkland, J.I., Deblieux, D.D., Madsen, S.K., Cavin, J., Milner, A.R.C. and Panzarin, L. 2010. New basal iguanodonts from the Cedar Mountain Formation of Utah and the evolution of thumb-spiked dinosaurs. PLoS ONE, 5:e14075.

Mclntosh, J.S. and Berman, D.S. 1975. Description of the palate and lower jaw of the sauropod dinosaur Diplodocus (Reptilia: Saurischia) with remarks on the nature of the skull of Apatosaurus. Journal of Paleontology, 49:187-199.

Nopcsa, F. 1900. Dinosaurierreste aus Siebenbürgen (Schädel von Limnosaurus transsylvanicus nov. gen. et spec.). Denkschriften der Kaiserlichen Akademie der Wissenschaften. Mathematisch-Naturwissenschaftliche Classe, 68:555-591.

Norman, D.B. 1980. On the ornithischian dinosaur Iguanodon bernissartensis from the Lower Cretaceous of Bernissart (Belgium). Bulletin-Institut Royale de Sciences naturelles de Belgique: Sciences de la Terre, 178:1-105.

Norman, D.B. 1984. On the cranial morphology and evolution of ornithopod dinosaurs. Symposia of the Zoological Society of London, 52:521-547.

Norman, D.B. 1986. On the anatomy of Iguanodon atherfieldensis (Ornithischia: Ornithopoda). Bulletin-Institut Royal des Sciences Naturelles de Belgique: Sciences de la Terre, 56:281-372.

Norman, D.B. 1990. A review of the Wealden iguanodontid dinosaur Vectisaurus valdensis (Hulke, 1874), with comments upon the status of the family Iguanodontidae, p. 147-161. In Carpenter, K. and Currie, P.J. (eds.), Dinosaur Systematics: Approaches and Perspectives.
Norman, D.B. 2002. On Asian ornithopods (Dinosauria: Ornithischia). 4. Probactrosaurus (Rozhdestvensky, 1966). Zoological Journal of the Linnean Society, 136:113-144.

Norman, D.B. 2004. Basal Iguanodontia, p. 413-437. In Weishampel, D.B., Dodson, P., and Osmólska, H. (eds.), The Dinosauria, $2^{\text {nd }}$ ed. University of California Press, Berkeley, California.

Norman, D.B., Crompton, A.W., Butler, R.J., Porro, L.B., and Charig, A.J. 2011. The Lower Jurassic ornithischian dinosaur Heterodontosaurus tucki Crompton \& Charig, 1962: cranial anatomy, functional morphology, taxonomy, and relationships. Zoological Journal of the Linnean Society, 163:182-276.

Norman, D.B., Sues, H., Witmer, L.M., and Coria, R.A. 2004a. Basal Ornithopoda, p. 393-412. In Weishampel, D.B., Dodson, P., Osmólska, H. (eds.), The Dinosauria, $2^{\text {nd }}$ ed. University of California Press, Berkeley, California.

Norman, D.B. and Weishampel, D. 1985. Ornithopod feeding mechanisms: their bearing on the evolution of herbivory. The American Naturalist, 126:151-164.

Oelrich, T. 1956. The anatomy of the head of Ctenosaura pectinata (Iguanidae). Miscellaneous Publications of the Museum of Zoology, University of Michigan, 94:1-168.

Ostrom, J.H. 1961. Cranial morphology of the hadrosaurian dinosaurs of North America. Bulletin of the American Museum of Natural History, 122:37-186.

Ostrom, J.H. 1970. Stratigraphy and paleontology of the Cloverly Formation (Lower Cretaceous) of the Bighorn Basin area, Montana and Wyoming. Peabody Museum of Natural History Bulletin, 35:1-234.

Parks, W.A. 1926. Thescelosaurus warreni: A new species of orthopodous dinosaur from the Edmonton Formation of Alberta. University of Toronto Studies. Geological Series, 21:1-42.

Prieto-Márquez, A. 2010. Global phylogeny of Hadrosauridae (Dinosauria: Ornithopoda) using parsimony and Bayesian methods. Zoological Journal of the Linnean Society, 159:435-502.

Rennison, C.J. 1996. The stable carbon isotope record derived from mid-Cretaceous terrestrial plant fossils from north-central Texas. Unpublished MS Thesis, Southern Methodist University, Dallas, Texas, USA.

Rozhdestvensky, A.K. 1966. New iguanodonts from Central Asia. Phylogenetic and taxonomic interrelationships of late Iguanodontidae and early Hadrosauridae. Paleontologicheskii Zhurnal, 1966:103-116.

Sanders, R. and Smith, D. 2005. The endocranium of the theropod dinosaur Ceratosaurus studied with computed tomography. Acta Palaeontologica Polonica, 50:601-616.

Schwarz, D.W.F. and Tomlinson, R.D. 1994. Physiology of the vestibular system p. 59-98. In Jackler, T.K. and Brackmann, D.E. (eds.), Neurotology, $1^{\text {st }}$ ed. Mosby, Philadelphia, Pennsylvania. 
Seeley, H. 1888. On the classification of the fossil animals commonly named Dinosauria. Proceedings of the Royal Society of London, 43:165-171.

Sereno, P. 1986. Phylogeny of the bird-hipped dinosaurs. National Geographic Research, 2:234-256.

Sereno, P.C. 1991. Lesothosaurus, "fabrosaurids," and the early evolution of Ornithischia. Journal of Vertebrate Paleontology, 11:168-197.

Sereno, P. 1997. The origin and evolution of dinosaurs. Annual Review of Earth and Planetary Sciences, 25:435-489.

Sereno, P., Wilson, J., Witmer, L., and Whitlock, J. 2007. Structural extremes in a Cretaceous dinosaur. PLoS ONE, 2:e1230.

Sternberg, C. 1940. Thescelosaurus edmontonensis, $\mathrm{n}$. sp., and classification of the Hypsilophodontidae. Journal of Paleontology, 14:481-494

Swofford, D. 2003. PAUP*. Phylogenetic Analysis Using Parsimony ( ${ }^{*}$ and Other Methods), Version 4.0.

Taquet, P. 1976. Géologie et paléontologie du gisement de Gadoufaoua (Aptien du Niger). Cahiers de Paléontologie. Éditions du Centre National de la Recherche Scientifique, p. 1-191.

Thulborn, R.A. 1970. The skull of Fabrosaurus australis, a Triassic ornithischian dinosaur. Palaeontology, 13:414-432.

Thulborn, R.A. 1972. The post-cranial skeleton of the Triassic ornithischian dinosaur Fabrosaurus australis. Palaeontology, 15:29-60.

Wang, $X$. and $X u, X$. 2001. A new iguanodontid (Jinzhousaurus yangi gen. et sp. nov.) from the Yixian Formation of western Liaoning, China. Chinese Science Bulletin, 46:1669-1672.

Wang, X., Pan, R., Butler, R.J., and Barrett, P.M. 2011. The postcranial skeleton of the iguanodontian ornithopod Jinzhousaurus yangi from the Lower Cretaceous Yixian Formation of western Liaoning, China. Earth and Environmental Science Transactions of the Royal Society of Edinburgh, 101:135-159.

Wedel, M.J., Cifelli, R.L., and Sanders, R.K. 2000. Sauroposeidon proteles, a new sauropod from the early Cretaceous of Oklahoma. Journal of Vertebrate Paleontology 20:109-114.
Weishampel, D.B. 1983. Hadrosaurid jaw mechanics. Acta Palaeontologica Polonica, 28:271-280.

Weishampel, D.B. 1984. The evolution of jaw mechanisms in ornithopod dinosaurs. Advances in Anatomy, Embryology and Cell Biology, 87:1-110.

Weishampel, D.B. 1985. An approach to jaw mechanics and diversity: the case of ornithopod dinosaurs. Fortschritte der Zoologie, 30:261-263.

Weishampel, D.B. and Bjork, P. 1989. The first indisputable remains of Iguanodon (Ornithischia: Ornithopoda) from North America: Iguanodon lakotaensis, sp. nov. Journal of Vertebrate Paleontology, 9:56-66.

Weishampel, D.B., Jianu, C.-M., Zoltan, C., and Norman, D.B. 2003. Osteology and phylogeny of Zalmoxes (n. g.), an unusual Euornithopod dinosaur from the latest Cretaceous of Romania. Journal of Systematic Palaeontology, 1:65-123.

Weishampel, D.B., Norman, D.B., and Grigorescu, D. 1993. Telmatosaurus transsylvanicus from the late Cretaceous of Romania: the most basal hadrosaurid dinosaur. Palaeontology, 36:361-385.

Wever, E.G. 1978. Order Crocodilia: The Crocodiles, p. 924-964. In The Reptile Ear: Its Structure and Function. Princeton University Press, Princeton, New Jersey.

Winkler, D.A., Murry, P.A., and Jacobs, L.L. 1990. Early Cretaceous (Comanchean) vertebrates of central Texas. Journal of Vertebrate Paleontology 10:95116.

Winkler, D.A., Murry, P.A., and Jacobs, L.L. 1997. A New Species of Tenontosaurus (Dinosauria: Ornithopoda) from the Early Cretaceous of Texas. Journal of Vertebrate Paleontology, 17:330-348.

Winkler, D.A., Murry, P.A., and Jacobs, L.L. 1998. The new ornithopod dinosaur from Proctor Lake, Texas, and the deconstruction of the family Hypsilophodontidae. Journal of Vertebrate Paleontology, 18:87A.

Witmer, L. and Ridgely, R. 2009. New insights into the brain, braincase, and ear region of Tyrannosaurs (Dinosauria, Theropoda), with implications for sensory organization and behavior. The Anatomical Record, 292:1266-1296. 


\section{APPENDIX 1. \\ TAXA AND SPECIMEN RESOURCE LIST}

The following is a list of taxa analyzed and compared in this study, with references to descriptive papers used in the analysis of each.

\section{Outgroup:}

Lesothosaurus diagnosticus Galton, 1978-Thulborn (1970, 1972), Santa Luca (1984), Weishampel and Witmer (1990a), Sereno (1991a), Norman, (2004)

\section{Basal Ornithopod Taxa:}

Hypsilophodon foxii Huxley, 1869-Galton (1974a), Norman (2004)

Parksosaurus warreni Parks, 1926-Parks (1926), Sternberg (1940), Galton (1973)

Thescelosaurus neglectus Gilmore, 1913-Gilmore (1913), Gilmore (1915), Sternberg (1940), Galton (1974b, 1995, 1997), Norman et al. (2004)

\section{Basal Iguanodontian Taxa:}

Camptosaurus dispar (Marsh, 1879)-Marsh (1879), Gilmore (1909), Norman (2004)

Cumnoria prestwichii Hulke 1880-Hulke (1880), Galton and Powell (1980), Norman (2004)

Dryosaurus altus (Marsh, 1878)-Marsh (1878), Galton (1983), Norman (2004)

Eolambia caroljonesa Kirkland, 1998-Kirkland (1998), Head (2001), Norman (2004), McDonald et al. (2012)

Iguanodon bernissartensis Boulenger, 1881-Boulenger (1881), Norman (1980), Norman (2004)

Jinzhousaurus yangi Wang and $\mathrm{Xu}, 2001-$ Wang and $\mathrm{Xu}$ (2001), Barrett et al., (2009), Wang et al. (2011)
Mantellisaurus atherfieldensis (Hooley, 1925)-Hooley (1925), Norman (1986), Norman (2004)

Ouranosaurus nigeriensis Taquet, 1976-Taquet (1976), Norman (2004)

Tenontosaurus dossi Winkler et al., 1997-Winkler et al. (1997), Norman (2004)

Tenontosaurus tilletti Ostrom, 1970-Ostrom (1970), Forster (1990), OMNH 58340 (subadult, primary specimen), OMNH 2531 (juvenile), OMNH 16562 (subadult, skull transversely compressed, palpebral), OMNH 34191 (premaxilla)

Zalmoxes robustus (Nopcsa, 1900)-Nopcsa (1900), Weishampel et al. (2003), Norman (2004)

\section{Hadrosauroid Taxa:}

Corythosaurus casuarius Brown, 1914-Brown (1914, 1916), Ostrom (1961), Horner et al. (2004)

Edmontosaurus regalis Lambe 1917-Lambe (1917), Lull and Wright (1942), Norman (2004), Horner et al. (2004)

Probactrosaurus gobiensis Rozhdestvensky, 1966Rozhdestvensky (1966), Norman (2002), Norman (2004)

Protohadros byrdi Head, 1998-Head (1998), Norman (2004)

Telmatosaurus transsylvanicus (Nopcsa, 1900)-Nopcsa (1900), Nopcsa (1903), Weishampel et al. (1993), Horner et al. (2004) 


\section{APPENDIX 2. CHARACTER DESCRIPTION}

The following is a list of character states used in this analysis. States were determined independently, but similarities with previously published analyses (Norman, 2004; McDonald et al., 2010) have been noted.

\section{Facial Characters:}

1. Presence of nasal contact with the maxilla and reason for exclusion of contact (Modified from Norman, 2004: Character 6):

1:0. Contact

1:1. No contact due to an anterior extension of the lachrymal

1:2. No contact due to a posterior extension of the premaxilla

2. Character of the occlusal margin of the premaxilla (Modified from McDonald et al., 2010: Character 33):

2:0. Smooth

2:1. Denticulate

2:2. Scooped, possibly to accommodate the denticles of the predentary

3. Premaxillary teeth (Modified from Norman, 2004: Character 3; McDonald et al., 2010: Character 29):

3:0. Present

3:1. Absent

4. Posterior extent of the nasal process of the premaxilla:

4:0. Does not reach antorbital fenestra

$4: 1$. Reaches antorbital fenestra or, if no fenestra present, posterior border of dorsal process of maxilla

5. Beak position relative to tooth row (Modified from Norman, 2004: Character 2):

5:0. At level

5:1. Below

6. Width of combined frontals compared to length (Modified from Norman, 2004: Character 15):

$6: 0$. About $2 / 3$ length of frontal

$6: 1$. About equal to length of frontal

$6: 2$. Twice length of frontal

7. Transverse length of frontals' suture with parietal:

$7: 0$. About equal with combined width of frontals

7:1. About two-thirds combined width of frontals

$7: 2$. About half combined width of frontals

7:3. About one-third combined width of frontals

8. Frontal contribution to dorsal orbital rim (Modi-

fied from Norman, 2004: Character 16;

McDonald et al., 2010: Character 68):

8:0. More than half
$8: 1$. Less than half

8:2. Does not contribute

9. Triangular process of the parietal:

9:0. Present

9:1. Absent

10. Ratio of axial lengths of the frontal and the nasal:

10:0. Roughly equal in length

$10: 1$. Nasal is one and one half times length of the frontal

$10: 2$. Nasal is twice length of the frontal

$10: 3$. Nasal is three times length of the frontal

10:4. Nasal is four times length of the frontal

11. Position of nasal contribution to the border of the external naris:

11:0. Posterodorsal border

11:1. Dorsal border

11:2. Does not contribute

12. Nature of the articulation of the nasal, frontal, and prefrontal:

12:0. Nasal overlaps frontal, overlapped by prefrontal

12:1. Nasal overlaps frontal

12:2. Nasal has transverse suture with frontal

12:3. Frontal overlaps nasal

13. Nature of the articulation of the premaxillae with the nasals:

13:0. Nasal processes wedge between nasals 13:1. Nasal overlapped by nasal process of premaxilla

13:2. Tip to tip

13:3. Nasals lie in beveled grooves in lateral surface of nasal processes of premaxilla

13:4. Nasals overlap nasal processes of premaxilla

13:5. Nasal processes split into dorsal and ventral branches, which overlap the nasals

14. Supratemporal fensestra imprints frontal:

14:0. Present

14:1. Absent

15. Lateral suture of the laterosphenoid with the:

15:0. Frontal and postorbital

15:1. Postorbital only

15:2. Frontal only

16. Rugose lateral edge of the postorbital:

16:0. Absent

16:1. Present

17. Parietal contact with the postorbital:

17:0. Present 
17:1. Absent

18. Ectopterygoid articulation with the palatine:

18:0. Present

18:1. Absent

19. Ectopterygoid articulation with the jugal (Modified from Norman, 2004: Character 14; McDonald et al., 2010: Character 53):

19:0. Present

19:1. Absent

20. Jugal articulation with the quadrate:

20:0. Absent

20:1. Present

21. Ventral lobe of the jugal (Modified from Norman, 2004: Character 13; McDonald et al., 2010: Character 56):

21:0. Absent

21:1. Present

22. Jugal extends farthest posteriorly in the:

22:0. Quadratojugal process

22:1. Postorbital process

23. Accessory foramen in the quadratojugal (Modified from Norman, 2004: Character 17; McDonald et al., 2010: Character 58):

23:0. Absent

23:1. Present

24. Enclosure of the quadratojugal by the jugal:

24:0. None

24:1. Dorsal

24:2. Dorsal and ventral

25. Quadrate foramen:

25:0. Absent

25:1. Present

26. Quadratojugal size (relative to quadratojugal process of jugal):

26:0. Reduced

26:1. Large

27. Position of the contribution of the lachrymal to the orbital border:

27:0. Anteroventral corner

27:1. Anterior border

27:2. Middle anterior border

28. Contact of the nasal with the lachrymal (Modified from McDonald et al., 2010: Character 50):

28:0. Present

28:1. Absent

29. Shape of the medial lamina of the palatine:

29:0. Fan

29:1. Rectangle

29:2. Triangle

30. Maxillary tooth family count along a single side

31. Maxillary diastema:

31:0. Absent

31:1. Present
32. Lateral process of the maxilla for articulation with the jugal:

32:0. Absent

32:1. Present

33. Jugal articulation with the maxilla:

33:0. Posterior on the latter element

33:1. Displaced forward on the latter element

34. Prefrontal contact with the premaxilla (Modified from McDonald et al., 2010: Character 37):

34:0. Absent

34:1. Present

35. Position of the prefrontal contact with the nasal: 35:0. Anteromedially

$35: 1$. Dorsally

36. Pterygoid contact with the shaft of the quadrate:

36:0. Absent

36:1. Present

37. Quadrate notch (Modified from McDonald et al., 2010: Character 61):

37:0. Absent

37:1. Present

38. Transversely wide ventral quadratic condyle (Modified from Norman, 2004: Character 18; McDonald et al., 2010: Character 64):

38:0. Present

38:1. Absent

39. "Hamalar" process of the head of the quadrate: 39:0. Absent

39:1. Present

40. Contact between paired squamosals on the midline (Modified from McDonald et al., 2010:

Character 67):

40:0. Absent

40:1. Present

41. Lateral visibility of anterior process of squamosal:

41:0. Largely obscured

41:1. Prominent across intertemporal bar

\section{Neurocranial Characters:}

42. Shape of occipital condyle:

42:0. Subspherical

42:1. Flattened/reniform

43. Supraoccipital participation in the foramen magnum (Modified from McDonald et al., 2010:

Character 69):

43:0. Present

43:1. Absent, due to the exoccipital

44. Shape of the cultriform process of the parasphenoid in ventral aspect:

44:0. Elongate

44:1. Triangular

45. Outline shape of the parietal in dorsal aspect: 
45:0. Rectangular

45:1. Triangular

46. Ratio of length to the width of the parietal:

46:0. 1:1

46:1. $1.5: 1$

46:2. $1: 1.5$

46:3. $1: 2$

47. Pronounced sagittal crest of the parietal:

47:0. Absent

47:1. Present

48. Interparietal Eminence:

48:0. Present

48:1. Absent

49. Parietal/prootic contact:

49:0. Present

49:1. Absent

50. Crista prootica is comprised of:

50:0. Laterosphenoid/prootic/opisthotic

50:1. Prootic/opisthotic

\section{Mandibular Characters:}

51. Dentary diastema (Modified from Norman, 2004: Character 20; McDonald et al., 2010: Character 9):

51:0. Absent

51:1. Present

52. Posteroventral corner of the dentary extends behind coronoid process:

52:0. Absent

52:1. Present

53. Dentary symphysis:

53:0. Set level with tooth row

53:1. Set below tooth row

54. Dorsoventral borders of dentary (Modified from Norman, 2004: Character 22; McDonald et al., 2010: Character 15):

54:0. Thicker posteriorly

54:1. Parallel

$54: 2$. Thicker anteriorly

55. Length of the dentary bearing dentition:

$55: 0$. Full

$55: 1.2 / 3$

55:2. Half

56. Orientation of coronoid process of the mandible (Modified from Norman, 2004: Character 23; McDonald et al., 2010: Character 20):

56:0. Posteriorly deflected

$56: 1$. Vertical

$56: 2$. Anteriorly deflected

57. Coronoid bone:

57:0. Present

57:1. Absent

58. Presence of two processes around the Meckelian canal:
58:0. Absent

58:1. Present

59. Presence of shelf between the teeth and the coronoid process (Modified from Norman, 2004: Character 24):

59:0. Absent

59:1. Present

60. Axially flared coronoid process (Modified from McDonald et al., 2010: Character 21):

60:0. Absent

60:1. Present

61. Bifurcate ventral process of the predentary (Modified from Norman, 2004: Character 19; McDonald et al., 2010: Character 2):

61:0. Absent

61:1. Present

62. Character of the occlusal margin of the predentary (Modified from McDonald et al., 2010: Character 4):

62:0. Smooth

62:1. Denticulate

63. Lateral visibility of the angular (Modified from Norman, 2004: Character 26; McDonald et al., 2010: Character 27):

63:0. Visible

63:1. Reduced visibility

63:2. Not visible

64. Nature of articulation of the angular with the surangular (Modified from McDonald et al., 2010: Character 26):

64:0. Angular laterally overlaps surangular

$64: 1$. Groove in ventral surangular

64:2. Angular laterally abuts surangular

65. Anterior extension of the coronoid:

65:0. Present

65:1. Absent

66. Lateral visibility of the coronoid bone:

66:0. Invisible

66:1. Largely visible

66:2. Reduced

67. Splenial coverage of the Meckelian canal:

67:0. Extensive

67:1. Posterior only

68. Presence of the prearticular:

68:0. Present

68:1. Absent

69. Number of surangular foramina (Modified from Norman, 2004: Character 25; McDonald et al., 2010: Character 24):

69:0. Two

69:1. Three

69:2. One

69:3. None 
70. Nature of ridges on the teeth (Modified from Norman, 2004: Character 27; McDonald et al., 2010: Characters $85,90,91$ ):

70:0. Absent

70:1. Ridge on Dentary teeth

70:2. Ridge on Dentary and Maxillary Teeth

70:3. Ridge on Maxillary with reduced Dentary ridge

70:4. Ridge on Dentary with reduced Maxillary ridge

71. Dentary tooth family count along a single side

\section{Postcranial Characters:}

Taken, with modifications, from Norman, 2004.

72. Dorsal neural spines:

72:0. Low and Square

$72: 1$. Rectangular and height more than twice width

72:2. Extremely elongate, height more than six times width

73. Sacrum:

73:0. Seven or fewer vertebrae

73:1. More than seven

74. Scapular blade:

74:0. Straight

74:1. Curved

74:2. Curved and flared distally

75. Scapular acromion:

75:0. Prominent boss on the anterior margin of the scapula

$75: 1$. Boss is reflected laterally

76. Humerus:scapula length:

76:0. Approximately equal lengths

$76: 1$. Scapula longer than humerus

77. Sternal shape:

77:0. Reniform

77:1. Hatchetlike

78. Carpal structure:

78:0. Fully ossified and blocklike

78:1. Reduced

79. Metacarpal I shape:

79:0. Dumbbell-like

79:1. Short and blocklike

79:2. Absent

80. Metacarpals II-IV:

80:0. Dumbbell-like and spreading

80:1. Closely appressed

$80: 2$. Appressed, slender and elongate

81. Manus digit I:

81:0. Present

81:1. Absent

82. Manus ungual $\mathrm{I}$ :

82:0. Claw-like

82:1. Conical
82:2. Absent

83. Manus unguals II and III:

83:0. Claw-like

83:1. Flattened, twisted and hoof-like

83:2. Digit II claw-like, Digit III nub

84. Manus digit III:

$84: 0$. Four phalanges

$84: 1$. Three phalanges

85. Preacetabular process of ilium:

85:0. Long and laterally compressed

85:1. Strongly downturned

85:2. Distally twisted

86. Dorsal margin of iliac blade:

86:0. Mostly smooth edged

$86: 1$. Strongly notched behind the ischial peduncle

87. Dorsal edge of ilium above ischial peduncle:

87:0. Not thickened and bevelled

87:1. Thickened

87:2. Everted with pendent tip

88. Ilium, postacetabular process:

88:0. Tapering posteriorly

88:1. Low and rectangular

89. Pubis, prepubic process:

89:0. Short and blunt

89:1. Elongate

90. Pubis, prepubic process:

90:0. Rod-shaped

90:1. Laterally compressed, bar-like

90:2. Short constriction and distal expansion

90:3. Deep expansion

91. Pubic shaft:

91:0. Ends adjacent to distal end of ischium

$91: 1$. Shorter than ischium, no pubic symphysis

92. Ischium, shaft shape:

92:0. Straight

92:1. Arched dorsally

93. Ischium shaft:

93:0. Flattened in cross section

93:1. Rounded in cross section

94. Obturator process of the ischium:

94:0. Absent

94:1. Present near midshaft

94:2. Present and close to pubic peduncle

95. Tip of ischium:

95:0. Unexpanded

95:1. Axial expansion to form a boot

96. Femur:

96:0. Distal half of shaft curved posteriorly

96:1. Straight

97. Femoral fourth trochanter:

97:0. Pendent

97:1. Triangular 
97:2. Crested eminence

98. Femur extensor groove:

98:0. Open shallow trough

98:1. U-shaped groove

98:2. Partially enclosed channel

98:3. Fully enclosed tunnel

99. Femur distal condyles:

99:0. Moderately expanded posteriorly

99:1. Expanded posteriorly and anteriorly

100. Metatarsal I:
100:0. Well developed and articulates with phalanges

100:1. Slender and splintlike

100:2. Absent

101. Pedal unguals:

101:0. Elongate and pointed claws

101:1. Elongate but bluntly truncated

101:2. Short, broad and crescentic with reduced or absent claw grooves 


\section{APPENDIX 3.}

The following is a matrix composed of the states coded for each of 19 ingroup taxa and one outgroup taxon analyzed for each of the characters in Appendix 2 (available online only palaeo-electronica.org/content/ 2015/1178-skull-of-tenontosaurus).

\section{APPENDIX 4}

The following is a collection of three-dimensional renderings of each of the elements of the skull of OMNH 58340. They are stored as stl files, which can be easily viewed using software freely available for download on the internet (e.g., by searching for "stl file viewer") (available online only palaeo-electronica.org/content/ 2015/1178-skull-of-tenontosaurus).

\section{APPENDIX 5.}

The following is the CT data generated for use in this study. The first section (Cranium) comprises scans of the skull, without the mandibles or disarticulated pieces. The second section (Braincase) comprises scans of the braincase section of the cranium, taken at a higher resolution than those for the rest of the skull. Finally, the third section (Miscellaneous) comprises the mandibles and other disarticulated elements (e.g., the right prefrontal and the predentary). Also included are documents detailing the scanning parameters used for each set of scans.(All files are available online only palaeo-electronica.org/content/2015/1178skull-of-tenontosaurus.) 